\title{
Copyright
}

by

Paul Holloway

2016 
The Dissertation Committee for Paul Holloway Certifies that this is the approved version of the following dissertation:

\section{Incorporating Movement in Species Distribution Models}

\section{Committee:}

Jennifer A. Miller, Supervisor

Eugenio Arima

Anthony Di Fiore

Timothy Keitt

Kenneth R. Young 
Incorporating Movement in Species Distribution Models

by

Paul Holloway, B.S.; M.S.

\author{
Dissertation \\ Presented to the Faculty of the Graduate School of \\ The University of Texas at Austin \\ in Partial Fulfillment \\ of the Requirements \\ for the Degree of \\ Doctor of Philosophy
}

The University of Texas at Austin

May 2016 


\section{Acknowledgements}

First and foremost I would like to thank my advisor Jennifer A. Miller. None of this would be possible without the insightful advice, steadfast support and substantial time Jennifer has given me throughout this process. From the initial discussions about returning to grad school, to the final edits of this dissertation, I cannot thank you enough for everything you have done to make these five years as academically and professionally rewarding as possible, while also making it an extremely enjoyable experience. I could not have asked for a better advisor, and I know I will continue value your advice, wisdom and friendship for many years to come. Thank you!

I would also like to thank my dissertation committee in their support throughout this process. Eugenio Arima, Anthony Di Fiore, Timothy Keitt and Kenneth R. Young have offered their unwavering support, perceptive advice and knowledge at every stage of this dissertation, often when I had appeared unannounced at their offices. Our discussions and your comments have improved this dissertation beyond anything I could have hoped to achieve and I am subsequently indebted to you all. Many thanks.

I am fortunate to be a part of the Spatial Sciences Lab. This is the most dynamic and enjoyable office environment I have ever worked in, and the productive discussions over \$1 Lone Stars on a Wednesday evening was an added and pleasant bonus. So for all that, I thank Thoralf Meyer, Thomas Christiansen, Molly Polk, Niti Mishra, and Brendan Hoover. I also have the fortune of being part of the Digital Landscape Lab, and I would like to emphasize my thanks Kelley Crews and Cody Schank for their support pertaining to both this research as well as in a more general sense. Similarly, the other members of the DLL have also been an ever-present source of support, Dan LeVine, Xuebin Yang, 
and Robert Bean. I am also especially grateful for the support and friendship from fellow graduate students (past and present) that have made the last five years at UT a pleasure. The Graduate Association of Geography and the Environment (GAGE) is a thriving organization to be a part of, so I thank everyone I have interacted with over the course of my tenure here, but in particular I thank John Clary, Justin Laue, André Celarino, Bisola Falola, Katherine Lininger, Christine Bonthius, Josh Rudow, Jon Gehrig, and Aaron Groth.

I would also like to thank everyone in the Department of Geography and the Environment. The department has afforded me several opportunities as both a student and an employee. I was fortunate to be given the opportunity to be Jennifer's Graduate Research Assistant for her 3-year National Science Foundation (\#0962198) project "Spatial Autocorrelation and Species Distribution Models: Analyzing the Effects of Spatial Structure, Sampling Strategy, Statistical Methods and Scale Using Simulated Data" . Similarly, I was given the opportunity to be the Teaching Assistant for GRG 301C "The Natural Environment" under Carlos Ramos-Scharrón, and then further develop my teaching as the Instructor of Record for GRG 335N "Landscape Ecology" and GRG 310C "Spatial Data and Analysis". These opportunities have surpassed any expectations I entered the program with, and I am thankful for all those who have worked with me towards that. The department is also a great place to partake in intellectual debates, with the meta-analysis in Chapter 1 a result of a discussion from my second semester in GRG 390L, so I thank Paul Adams and my cohort for that. Finally James Gunter deserves special thanks for all his help with the seemingly endless number of administrative matters I have concerned him with over the last 5 years. 
I was also fortunate to receive financial support from a number of fellowships. Without these awards, I would not have been able to complete this research. In total, I was awarded approximately $\$ 45,000$ from the following sources: University of Texas at Austin, Graduate School Named / Endowed Continuing Fellowship (\$38,000 including tuition and fees); University of Texas at Austin, Graduate School Recruitment Fellowship $(\$ 6,000)$; University of Texas at Austin, Department of Geography and the Environment Robert E. Veselka Endowed Fellowship (\$950).

My dissertation would not have been possible without the incredible datasets I have used. I am therefore incredibly grateful to the researchers who have given me permission to utilize their data to answer my own research questions. Similarly, my analysis in $\mathrm{R}$ and NetLogo has been improved by the presence of the many online forums, so I therefore thank the online communities. Following this, where possible I have included data or code in the appendices so that future researchers working in a similar field may benefit like I have. In particular, I would like to thank Simon Gillings at the British Trust for Ornithology who granted me initial access to the 2007-2011 British breeding bird atlas and then later collaborated on the research presented in Chapter 2 . Furthermore, I would like to thank the thousands of volunteer participants in the bird atlas projects whose data made the analysis possible. I would like to thank Jack Williams and Tom Webb III for providing access to and discussing the fossilized pollen data and paleoclimate data used in Chapter 3. I would also like to thank Glyn Maude for providing the brown hyena telemetry data used in Chapter 4, and Richard Holland, Martin Wikelski, Franz Kümmeth and Carlos Bosque for making their oilbird telemetry data accessible on Movebank. 
The UK Climate Projections data were made available by the Department for Environment, Food and Rural Affairs (Defra) and Department for Energy and Climate Change (DECC) under license from the Met Office, Newcastle University, University of East Anglia and Proudman Oceanographic Laboratory. These organizations accept no responsibility for any inaccuracies or omissions in the data, nor for any loss or damage directly or indirectly caused to any person or body by reason of, or arising out of, any use of this data. The elevation data are available from the US Geological Survey, and along with the MODIS Land Cover Product were retrieved from the online Data Pool, courtesy of the NASA Land Processes Distributed Active Archive Center (LP DAAC), located at USGS/EROS, Sioux Falls, SD, http://lpdaac.usgs.gov. This dissertation incorporates data from the HydroSHEDS database which is (C) World Wildlife Fund, Inc. (2006-2013) and has been used herein under license. WWF has not evaluated the data as altered and incorporated within this dissertation, and therefore gives no warranty regarding its accuracy, completeness, currency or suitability for any particular purpose. Portions of the HydroSHEDS database incorporate data which are the intellectual property rights of (C) USGS (2006-2008), NASA (2000-2005), ESRI (1992-1998), CIAT (2004-2006), UNEPWCMC (1993), WWF (2004), Commonwealth of Australia (2007), and Her Royal Majesty and the British Crown are used under license. The HydroSHEDS database and more information are available at http://hydrosheds.org.

Finally, I thank my family. My mother, Julia Holloway, has always supported and encouraged me in everything I do, and these last five years have been no exception. My father, Eddie Holloway, has also provided me with enthusiasm and support at every step of the way. The good friendship of my brother, Michael Holloway, has always assisted me in everything I do, this dissertation being no exception. I'd like to thank my extended 
family Linda Sanders and Augusto Cruzado for everything they've done for me in the last few years. Finally, I thank Anna Cruzado Sanders, my wife. Somewhere in the midst of this dissertation I got married to my best friend, who has provided me with unfaltering support at every stage of these last five years. The excursion into the jungle on our honeymoon to observe oilbirds perhaps best sums up Anna's enormous support which has made all this possible. So, finally, thank you Anna! 


\title{
Incorporating Movement in Species Distribution Models
}

\author{
Paul Holloway PhD \\ The University of Texas at Austin, 2016
}

Supervisor: Jennifer A. Miller

\begin{abstract}
Climate change and concomitant urbanization have led to many species shifting their geographical distribution, while other species have simply gone extinct. Understanding the current and future distributions of species is therefore a critical component of biodiversity conservation, with species distribution models (SDMs) a powerful GIScience approach increasingly used to achieve this. Movement is an ecological process that influences the distribution of all species. Broad-scale (spatially and temporally) movement includes processes like dispersal and migration that determine whether newly suitable habitats are accessible, while fine-scale movement effects resource availability, and subsequently habitat suitability. In spite of this ecological significance, movement is rarely incorporated in SDMs. An increasingly important application of SDM is to study the effects of climate change on species distributions, and while several models that incorporate species dispersal abilities have been proposed, none have been tested or compared. Past data (British birds and North American flora) were used to calibrate and extrapolate species-environment relationships to the current timeperiod in order to assess the accuracy of these dispersal models. Significant differences in the accuracy and area projected as present by the dispersal models were identified, and
\end{abstract}


moreover, results were substantially influenced by the scale at which SDMs were calibrated. Fine-scale regular movement behaviors are another important determinant of mobile species distributions that are not currently incorporated within SDM. Spatial simulation was used to model the dynamic relationship between movement and biotic resources for oilbirds in Venezuela, in order to generate a new environmental variable for use in model calibration. The use of this layer greatly improved the accuracy and ecological realism of the SDM projection compared to other commonly applied SDM scenarios. Finally, the incorporation of movement across multiple scales has not been addressed in SDM research. Broad-scale dispersal was combined with fine-scale regular movements to predict continental changes in oilbird distribution over a decade, which improved the ecological understanding of distribution shifts and identified a number of new conceptual and methodological limitations. The incorporation of movement should now be a compulsory aspect of any study projecting the current or future distributions of species. 


\section{Table of Contents}

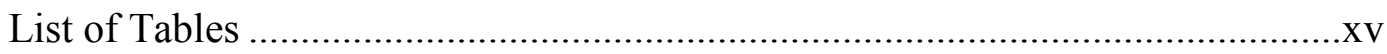

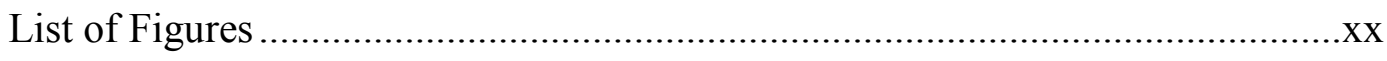

CHAPTER 1: A SYNTHESIS OF THE MOVEMENT CONCEPTS USED WITHIN SPECIES DISTRIBUTION MODELLING ...............................................................................1

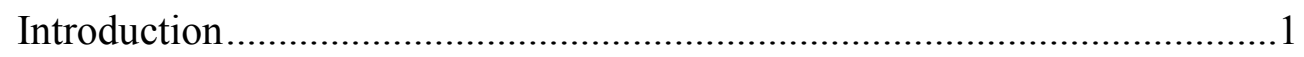

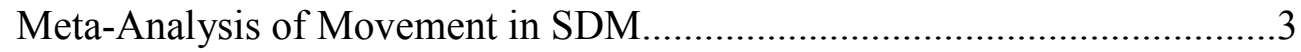

Movement Terminology .............................................................................6

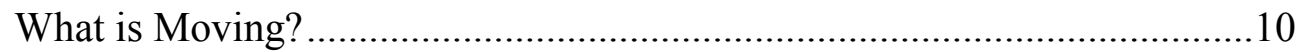

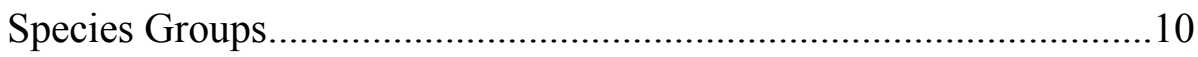

Individuals, Populations or Species .....................................................11

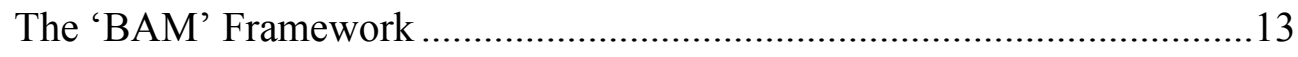

Applications of Movement In SDM …………………………………......16

Track Changing Environmental Conditions .......................................18

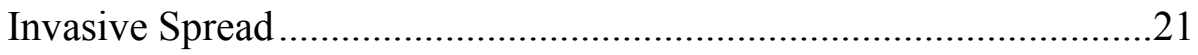

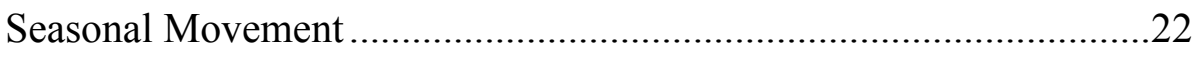

Regular Movements .....................................................................24

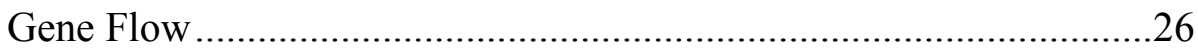

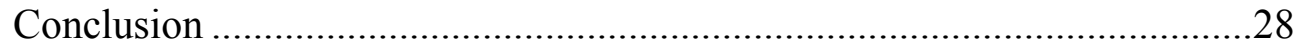

CHAPTER 2: INCORPORATING MOVEMENT IN SPECIES DISTRIBUTION MODELS: HOW DO SIMULATIONS OF DISPERSAL AFFECT THE ACCURACY AND UNCERTAINTY OF PROJECTIONS? ...............................................................................................29

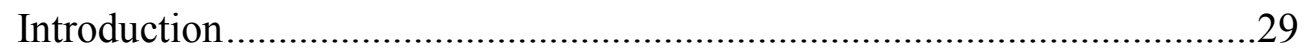

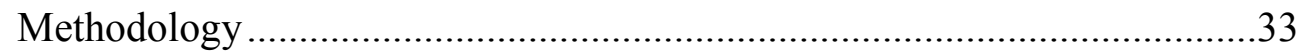

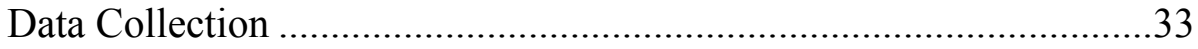

Species Distribution Modelling …………………………………........34

Statistical Modelling - Abiotic Suitability ……………………......35

Dispersal Modelling - Movement Suitability.................................37 


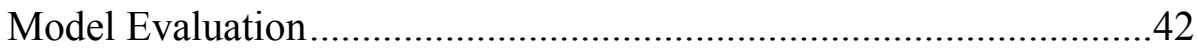

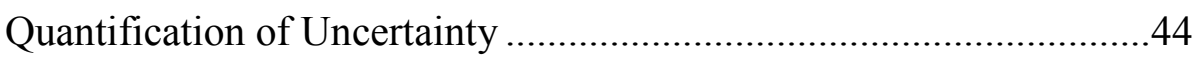

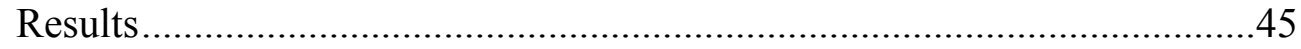

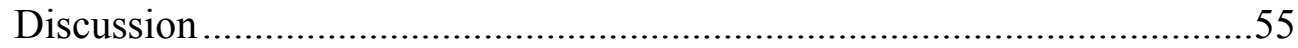

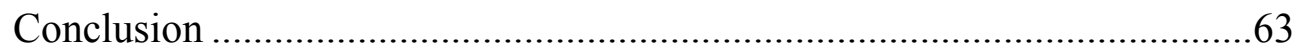

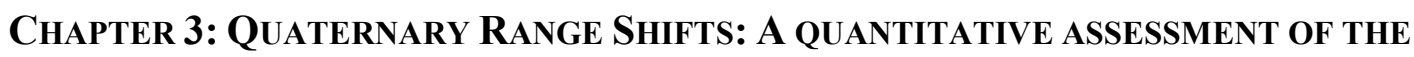
METHODS USED TO MODEL PLANT DISPERSAL ................................................65

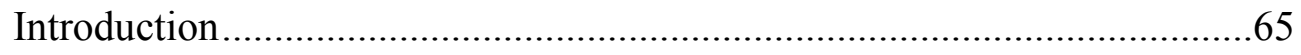

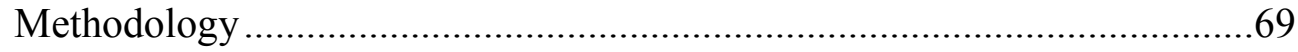

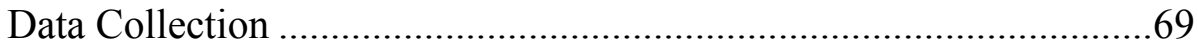

Species Distribution Modelling ……………………………............. 70

Statistical Modelling .................................................................... 71

Dispersal Modelling ............................................................... 72

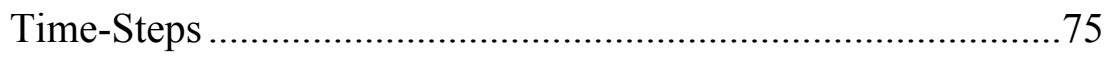

Model Accuracy ………………………………………….....

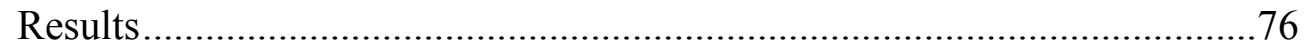

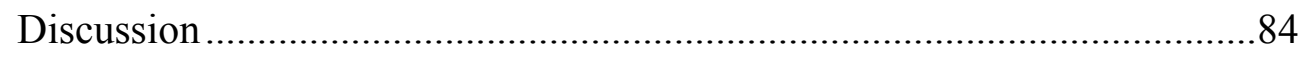

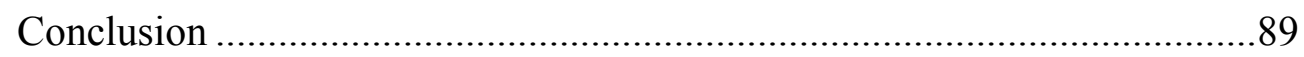

ChAPTER 4: UnCERTAINTY ANALYSIS OF STEP-SELECTION FUNCTIONS: THE EFFECT OF MODEL PARAMETERS ON INFERENCES ABOUT THE RELATIONSHIP BETWEEN ANIMAL MOVEMENT AND THE ENVIRONMENT .............................91

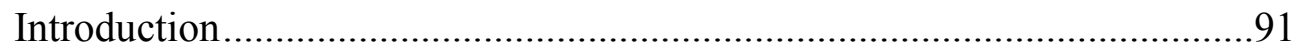

Methods of Generating Available Steps ………………………….....93

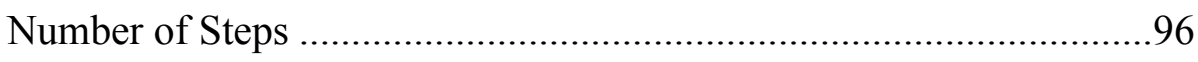

Modelling Approach …………………………………………......96

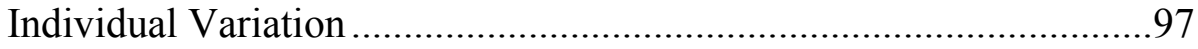

Uncertainty Analysis......................................................................97

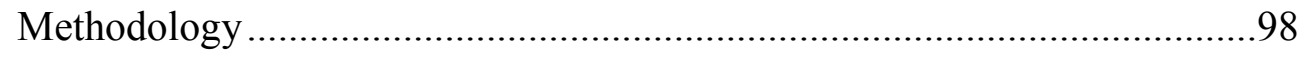

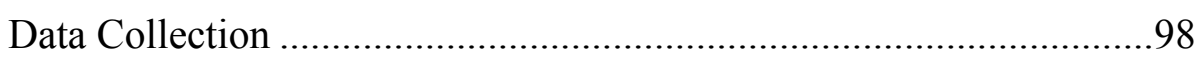

Uncertainty Analysis: Generating Available Steps...............................98 
Uncertainty Analysis: Number of Steps ........................................99

Uncertainty Analysis: Modelling Approach .....................................99

Uncertainty Analysis: Individual Variation ......................................99

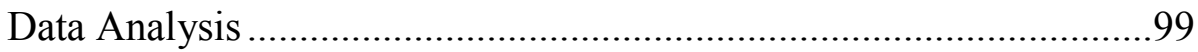

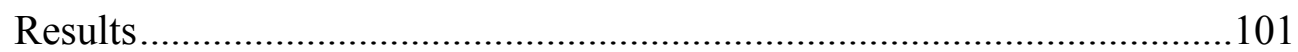

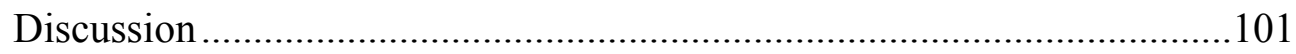

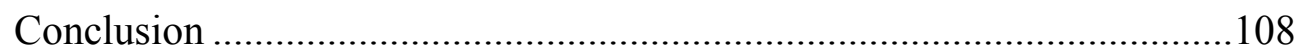

CHAPTER 5: INCORPORATING MOVEMENT-RELATED RESOURCE DYNAMICS INTO SPECIES DISTRIBUTION MODELLING ........................................................110

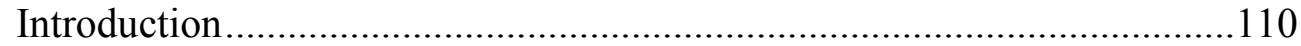

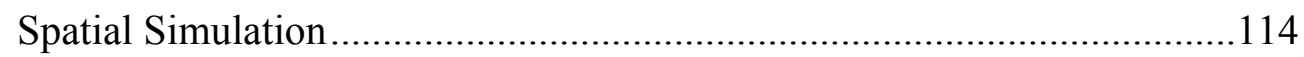

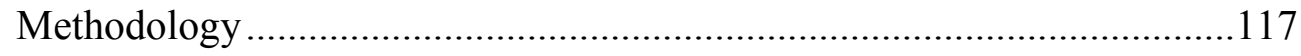

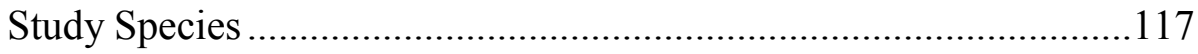

Species Distribution Modelling .....................................................119

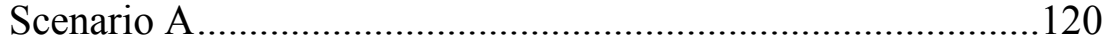

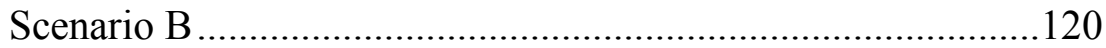

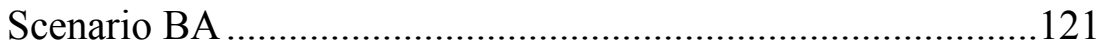

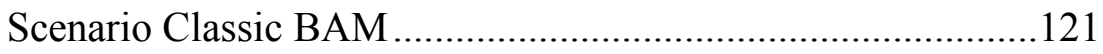

Scenario Dynamic BAM................................................... 122

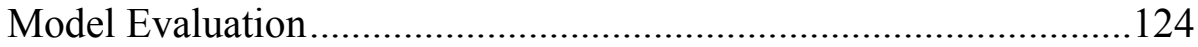

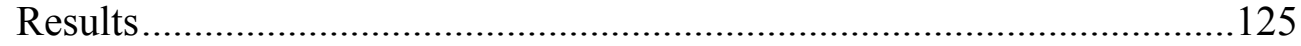

Step-Selection Function ....................................................... 125

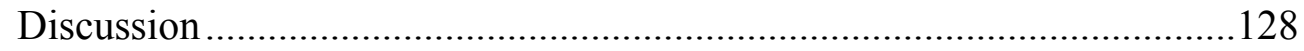

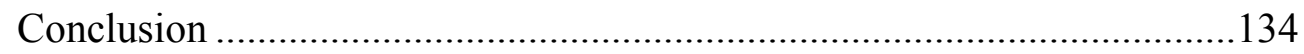

CHAPTER 6: MOVEMENT ACROSS SCALES: INCORPORATING BOTH DISPERSAL AND REGULAR MOVEMENTS IN SPECIES DISTRIBUTION MODELS.......................135

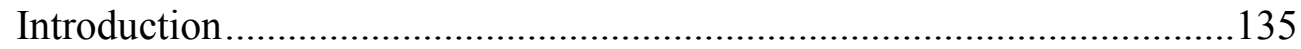

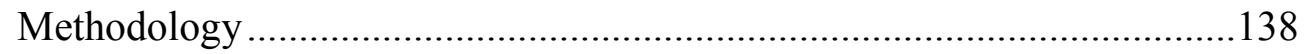

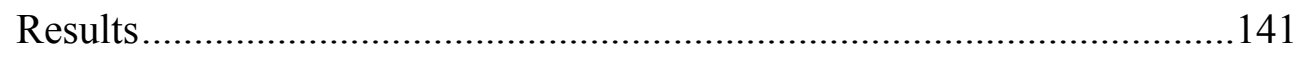

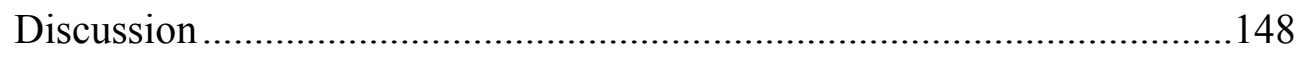


CHAPTER 7: INCORPORATING MOVEMENT IN SPECIES DiSTRIBUTION MODELS: A

CONCLUSION ...........................................................................................152

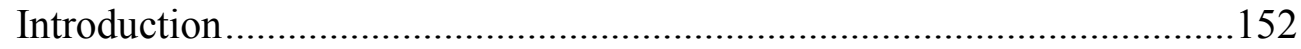

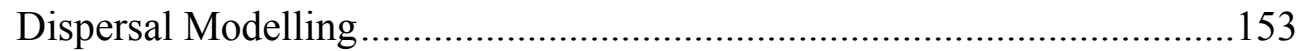

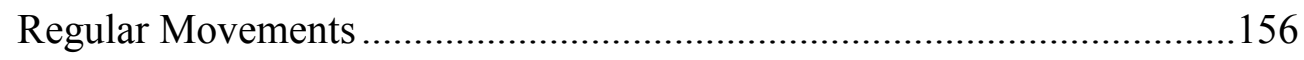

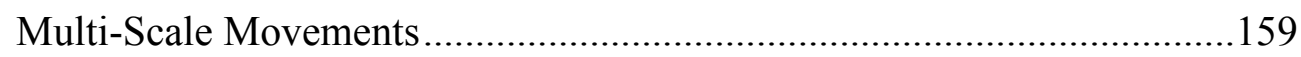

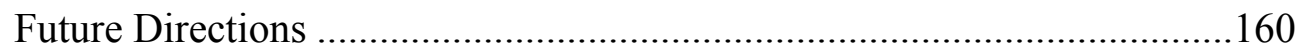

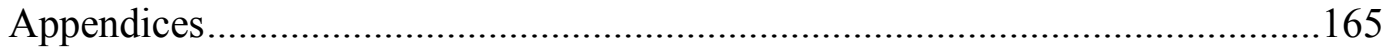

Appendix 1: Meta-Analysis ...................................................................165

Appendix 2: R Functions for Dispersal Models ..........................................166

Appendix 3: Final Regression Models For Bird Dispersal Distances ........172

Appendix 4: Dispersal Distances for Current North American Flora..........173

Appendix 5: Overview, Design Concepts and Details (ODD) .....................185

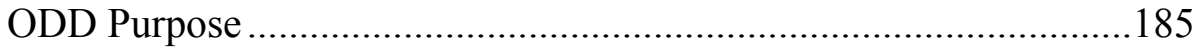

ODD State Variables and scales ......................................................185

ODD Process Overview and Scheduling ...........................................186

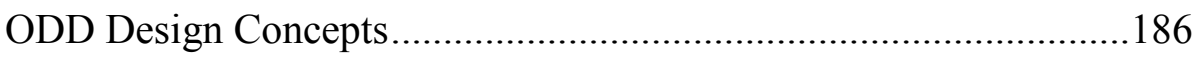

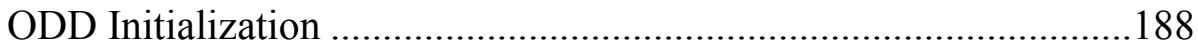

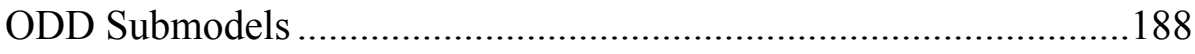

Step-Selection Function ………………………………………....188

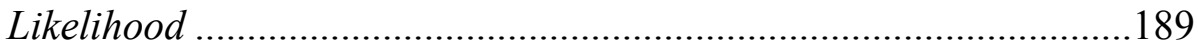

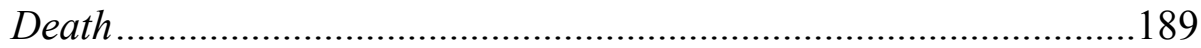

Appendix 6: NetLogo Code .....................................................................190

NetLogo World ..........................................................................190

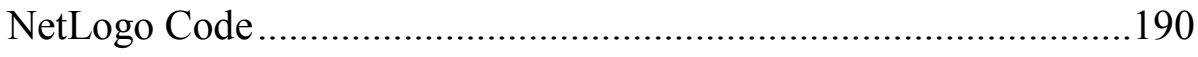

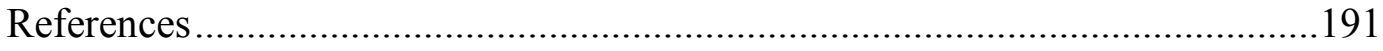




\section{List of Tables}

Table 1.1: The number of articles returned from different ISI Web of Knowledge search parameters. Terms representing the correlative speciesenvironment models are species distribution models (SDM), niche models (NM), predictive habitat distribution models (PHDM), and predictive vegetation models (PVM) ……………………….............

Table 1.2.: The number of times dispersal or migration was used to refer to the tracking of changing environmental conditions or regular movement. The value in the brackets refers to the number of times the main terms were indistinguishable. ..8

Table 1.3: The definitions of the main movement terms used throughout this dissertation. Terms are broadly adapted from Hansson and Åkesson (2014)

Table 1.4: The different dispersal models used to simulate movement in response to changing environmental conditions. 20

Table 2.1: The 20 methods used in this study and their reference. 38

Table 2.2: Threshold-dependent accuracy measures for species presence/absence models. $\mathrm{TP}=$ True Positive, $\mathrm{TN}=$ True Negative, $\mathrm{FP}=$ False Positive, $\mathrm{FN}=$ False Negative. 43 
Table 2.3: Mean and standard deviation (SD) of accuracy metric scores for the three statistical methods fitted on the 1990 environmental data and projected using the 2010 environmental data, with the assumption of unlimited dispersal across the 50 species. Abbreviations stand for Proportion Correctly Classified (PCC), True Skill Statistic (TSS) and Area Under the Curve (AUC) ....................................................46

Table 2.4: Pairwise comparison of the performance of the 20 dispersal methods. The value represents the average change in area $\left(\mathrm{km}^{2}\right)$ predicted present averaged for the 50 species between the method in the row compared to the method in the column. Green represents a significant increase in accuracy (True Skill Statistic - TSS) and red represents a significant decrease in accuracy (TSS) between the methods. Significance of $\alpha=$ 0.05 calculated using a paired sample t-Test. XD is no dispersal, $\mathrm{L}$ is limited dispersal $(1=$ rook's, $2=$ Queen's $)$, FR is fixed rate $(1=1$ step arithmetic, $2=1$ step geometric, $3=2$ step arithmetic, $4=2$ step geometric), DK is discrete kernel, PK is probabilistic kernel, UD is unlimited dispersal. For Kernels, $1=$ Gaussian Probability Density Function (PDF) arithmetic, 2 = Gaussian PDF geometric, 3 = inverse exponential PDF arithmetic, 4 = inverse exponential PDF geometric, $5=$ Cauchy PDF arithmetic, 6 = Cauchy PDF geometric. 
Table 2.5: Ensemble Agreement Index (EAI) scores for the 50 species. A value of 1 indicates all 20 methods made the same predictions for all locations. $-1 \mathrm{k}$ represents a significant lepokurtic distribution. - pk represents a significant platykurtic distribution. A kurtosis value twice the standard error was considered significantly different from a normal distribution.

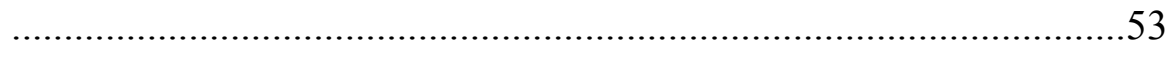

Table 3.1: The temporal resolution of each time-step implementation .............75

Table 3.2: The mean and standard deviation of the accuracy values recorded for models projected from $12 \mathrm{ka} \mathrm{BP}$ to $0 \mathrm{ka} \mathrm{BP}$ with an assumption of unlimited dispersal. Test data for the future projections consisted of every observation in North America (UD_ALL) and 1000 randomly generated points (UD_1000). Data were partitioned into 80:20 training:test data for the 12ka BP model (see Fielding and Bell 1997), although final genera-environment relationships were fit on all observations. Accuracy metrics of area under the curve (AUC), sensitivity, specificity, proportion correctly classified (PCC), true skill statistic (TSS) and Kappa were recorded.

Table 3.3: Values represent $80^{\text {th }}$ percentile of mean and maximum dispersal distances, and mean age of maturity. 
Table 3.4: The area $\left(\mathrm{km}^{2}\right)$ predicted present by each dispersal model implemented at different time steps (TS). Dispersal models include no dispersal (XD), limited dispersal with four-neighbor connectivity (L1), limited dispersal with eight-neighbor connectivity (L2), fixed rate (FR), discrete dispersal kernel (DK), probabilistic dispersal kernel (PK), and unlimited dispersal (UD). * Significantly different in area to UD $\alpha=0.01 . \Delta$ Significantly different to TS $1 \alpha=0.01$. Significance was calculated using matched paired t-tests. 83

Table 4.1: User-decisions in recently published step-selection function studies.94

Table 4.2: Information about the environmental variables used in the regression models. The IGBP classification scheme was used for the MODIS land cover product (LP DAAC 2013), which delineates into 16 classes. 100

Table 4.3: Wilcoxon matched pairs signed rank for hyenas, value refers to second group, *medium effect, **large effect. 103

Table 4.4: Wilcoxon matched pairs signed rank for oilbirds, value refers to second group, *medium effect, **large effect. 104

Table 5.1: $\quad$ BAM Scenarios used to explore oilbird distributional patterns......123

Table 5.2: The results of the conditional logistic regression model used for stepselection function scores. * significant at $\alpha<0.1, * *$ significant at $\alpha<$ 0.05 . 125

Table 5.3: The three accuracy metrics of the five BAM scenarios: Lowest possible threshold (LPT), minimum predicted area (MPA), and the Boyce index (BI) 127 
Table A3: Final regression models of the relationship between log body size and both arithmetic and geometric mean natal dispersal distances. Model 1 treats all 50 species as statistically equal, while Model 2 incorporates differences in species dispersal and origin. **significant at $0.01, *$

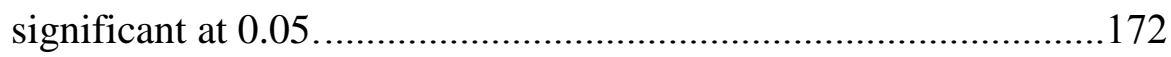

Table A4: Dispersal distance data for North American flora. ..........................173

Table A5: The results of the conditional logistic regression models used for stepselection function. * significant at $\alpha<0.1, * *$ significant at $\alpha<0.05 .188$ 


\section{List of Figures}

Figure 1.1: The 'BAM' diagram which depicts the interaction between biotic (B), abiotic (A), and movement (M) factors. $\mathbf{G}$ is the geographic space within which the analysis occurs, $\mathbf{G}_{\mathbf{a}}$ represents the abiotically suitable area, $\mathbf{G}_{\mathbf{i}}$ $\mathrm{s}$ the invadable (abiotic and biotic) suitable area. Finally $\mathbf{G}_{\mathbf{0}}$ represents the occupied (abiotic and biotic) suitable area and is therefore the actual distribution. Modified from Soberón (2007). .3

Figure 1.2: The number of SDM articles per year in which movement was implemented, or discussed but not implemented since 2005 (n.b. two SDM articles pre-2005 discussed movement but were not included in the above figure).

Figure 1.3: The number of SDM studies applying movement for a species group.11

Figure 1.4: Different 'BAM' scenarios, a) represents the classic 'BAM' framework as depicted already, $b$ ) represents a situation where all the combined suitable abiotic and biotic area is accessible, so all the invadable area is null, c) represents a situation where all of $\mathbf{A}$ and $\mathbf{B}$ are accessible, and so movement does not restrict the distribution. Finally d) represents a situation where neither $\mathbf{A}$ or $\mathbf{B}$ is accessible. This could represent the potential area of an invasive species, or where potentially abiotic and biotic conditions have shifted under climate change, but the species lacks the dispersal capacity required to keep track. Modified from Peterson et al. (2011). .15

Figure 1.5: The spatiotemporal scales of movement applications incorporated within SDM 
Figure 2.1: Conceptual diagram of workflow

Figure 2.2: Sensitivity scores for the 20 dispersal models for the three statistical methods and four breeding groups. XD is no dispersal, $\mathrm{L}$ is limited dispersal $(1=$ rook's, $2=$ Queen's $)$, FR is fixed rate $(1=1$ step arithmetic, $2=1$ step geometric, $3=2$ step arithmetic, $4=2$ step geometric), DK is discrete kernel, PK is probabilistic kernel, UD is unlimited dispersal. For Kernels, 1 = Gaussian PDF arithmetic, 2 = Gaussian PDF geometric, 3 = inverse exponential PDF arithmetic, $4=$ inverse exponential PDF geometric, $5=$ Cauchy PDF arithmetic, $6=$ Cauchy PDF geometric (n.b. DK1 to DK6 are consolidated under DK due to no visible variation). .48

Figure 2.3: Number of times each dispersal model recorded the highest value for a particular accuracy metric for each individual species. Across the 50 species and five accuracy metrics, the highest possible value could have been 250 .

Figure 2.4: Ensemble maps of predicted presence based on environmental suitability (using MaxEnt) and the 20 dispersal models for 2010 for $\mathrm{a}-\mathrm{c}$ ) three species with high uncertainty (Cetti's Warbler, Firecrest, Woodlark), df) three introduced species (Egyptian Goose, Ring-necked Parakeet, Redlegged Partridge) and g-i) three with high modelling certainty (Blackcap, Green Woodpecker, Great Spotted Woodpecker).

Figure 3.1: Flow chart illustrating the method behind using multiple dispersal steps in the SDM framework. .68 
Figure 3.2: Dispersal kernels for the six dispersal types recorded for the genera in the study. o represents mean dispersal distances, $\mathrm{x}$ represents maximum dispersal distances. Outlier of $30,000 \mathrm{~m}$ is missing from the trichometeorochory column. .79

Figure 3.3: Boxplots showing the range of True Skill Statistic scores (TSS) for dispersal models with the same time-step (TS) implementation (Top Panel) and for dispersal models with different time-step implementations (Bottom Panel). Dispersal models include no dispersal (XD), limited dispersal with four-neighbor connectivity (L1), limited dispersal with eight-neighbor connectivity (L2), fixed rate (FR), discrete dispersal kernel (DK), probabilistic dispersal kernel (PK), and unlimited dispersal (UD).

Figure 3.4: The projected distribution of Abies for each of the six dispersal models (rows) and each of the six time-step implementations (columns). Dispersal models include no dispersal (XD), limited dispersal with fourneighbor connectivity (L1), limited dispersal with eight-neighbor connectivity (L2), fixed rate (FR), discrete dispersal kernel (DK), and probabilistic dispersal kernel (PK). 82

Figure 3.5: Area $\left(\mathrm{km}^{2}\right)$ predicted as present averaged across 29 genera for dispersal models and time-step implementations .83

Figure 3.6: Abiotic suitability of Abies projected at 1,000 year intervals. .87 
Figure 4.1: Conceptual depiction of step-selection function. Red dots represent successive telemetry locations of an individual, with red arrows representing observed steps. The blue arrows represent the available steps that the individual could have taken. .92

Figure 4.2: $\quad \beta$ values for savanna and shrublands for individual and population modelling of hyena SSF (red) and evergreen broadleaf forest for oilbirds (blue) 102

Figure 4.3: $\beta$ values for savanna for hyenas (red) and evergreen broadleaf forest for oilbirds (blue) based on the four methods of generating step lengths. 102

Figure 4.4: $\beta$ values of shrublands for individual hyenas (red) and the $\beta$ values of evergreen broadleaf forest for individual oilbirds (blue). 106

Figure 5.1: Oilbirds at Quiscarrumi Bridge, Peru, December 2014. Photo by Paul Holloway 118

Figure 5.2: Movement trajectories for movement steps of an oilbird from a) random walk model, b) correlated random walk (parameterized on empirical turn angle and step length), c) biased correlation random walk (parameterized on step-selection function. d) the landscape of the grid 126

Figure 5.3: Spatial distribution of oilbirds in Venezuela from the five BAM scenarios. Threshold chosen as the proportion of presences compared to pseudo-absences. 129 
Figure 5.4: The data layers which represent biotic resources and movement factors a) the number of plant species present at a $1 \mathrm{~km}$ resolution, b) The number of plant species present within a $50 \mathrm{~km}$ focal area at a $10 \mathrm{~km}$ resolution, and c) the number of oilbirds that can be sustained within a $50 \mathrm{~km}$ focal area (the dynamic $\mathbf{B}$ and $\mathbf{M}$ layer).

Figure 6.1: Conceptual diagram of the four possible scenarios associated with biotic resources in future time. 137

Figure 6.2: Areal extents predicted as present by varying dispersal models and BAM scenarios 142

Figure 6.3: Areal extent (blue), sensitivity (orange) and specificity (of random background points - gray) of different dispersal models for a) Scenario A, b) Scenario cBAM, c) Scenario dBAM. 143

Figure 6.4: Ensemble model of 2010 oilbird distribution based on three BAM scenarios and four dispersal models. 145

Figure 6.5: Ensemble models and Ensemble Agreement Index (EAI) for the three BAM scenarios separated by dispersal model ..... 146

Figure 6.6: Ensemble models and Ensemble Agreement Index (EAI) for the four dispersal models separated by BAM scenarios 147

Figure A6: Screenshot of NetLogo World 190 


\section{CHAPTER 1: A SYNTHESIS OF THE MOVEMENT CONCEPTS USED WITHIN SPECIES DISTRIBUTION MODELLING}

\section{INTRODUCTION}

Climate change and concomitant urbanization have led to many species shifting to higher latitudes and altitudes globally, while many other species have simply gone extinct (Smith et al. 1993, Chen et al. 2011). Understanding the current and future distributions of species is therefore a critical component of biodiversity conservation. By identifying the factors most important in determining a species distribution, predictions can be made about its resilience, and more accurate estimations of range expansion, constriction, and maintenance can be made. Species distribution models (SDMs) are a powerful spatial ecological tool for studying the geographic distribution of plants, animals, and other biogeographic phenomena such as vector-borne diseases (Franklin 2009). This modelling framework provides a robust methodology for researchers and practitioners seeking to quantitatively assess the relationship between species distributions and environmental factors, and they have been used across an array of disciplines in order to test biogeographical hypotheses (Bolker et al. 2009), support nature reserve network design (Araújo et al. 2004), identify areas of rare species occurrence (Engler et al. 2004), assess species invasion risk (Medley 2010), and assess the impact of climate change on species (Wiens et al. 2011).

The 'BAM' framework (Figure 1.1) was developed by Soberón and Peterson (2005) and illustrates the individual and joint effects of the three most important types of factors that determine species distributions: biotic (B), abiotic (A), and movement (M). Biotic factors represent interactions with other species (i.e. competition, herbivory), abiotic factors represent the physiological tolerances of a species (i.e. temperature, 
precipitation) and movement factors refer to the processes that lead to an area being accessible to a species within a relevant timeframe (e.g., via dispersal or migration). $\mathbf{G}$ represents the geographic space within which the analysis occurs (e.g. study area), $\mathbf{G}_{\mathbf{a}}$ represents the abiotically suitable area, and $\mathbf{G}_{\mathbf{i}}$ is the invadable (abiotic and biotic) suitable area, where a species could exist if it could move there. Finally, $\mathbf{G}_{\mathbf{0}}$ represents the occupied (abiotic and biotic) suitable area and is therefore the actual distribution. While debate exists as to whether $\mathbf{G}_{\mathbf{a}}$ or $\mathbf{G}_{\mathbf{i}}$ represents the potential distribution (see Peterson et al. 2011 for a discussion), it is the area where a species could exist but is not found. Central to this framework is $\mathbf{M}$, as the inclusion of movement allows the species range to be considered a dynamic entity, as it is the inaccessibility to $\mathbf{G}_{\mathbf{i}}$ that is preventing an area from being occupied. While the importance of abiotic and biotic factors is well established in SDM applications; the incorporation of movement has lagged.

Movement is a pervasive feature of life, with every organism on the planet moving at some point in their life cycle, varying substantially across taxa, landscapes and individuals. By not implementing measures of movement within SDM, projections of species distributions ignore one of the most important determinants responsible for the patterns of current and future geographic ranges of species. Therefore, the aim of this dissertation is to address the longstanding issue of how to incorporate movement at different spatial and temporal scales into species distribution models. This should provide researchers, conservationists, and practitioners with a framework for better implementing multiple scales of movement in SDM. The remainder of this chapter will consist of a quantitative meta-analysis of SDM and movement. This synthesis will identify how movement has been incorporated in SDM to date, what are the under-studied components of incorporating movement, and identify emerging research frontiers. 


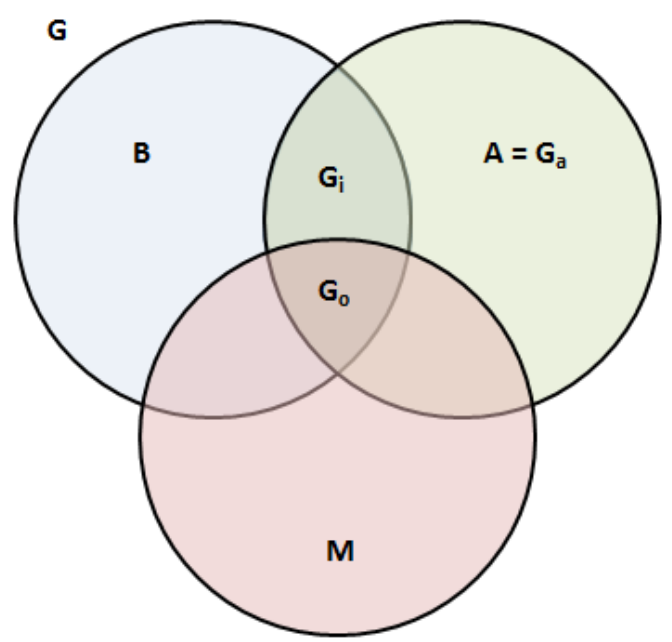

Figure 1.1: The 'BAM' diagram which depicts the interaction between biotic (B), abiotic (A), and movement (M) factors. $\mathbf{G}$ is the geographic space within which the analysis occurs, $\mathbf{G}_{\mathbf{a}}$ represents the abiotically suitable area, $\mathbf{G}_{\mathbf{i}} \mathbf{S}$ the invadable (abiotic and biotic) suitable area. Finally $\mathbf{G}_{\mathbf{0}}$ represents the occupied (abiotic and biotic) suitable area and is therefore the actual distribution. Modified from Soberón (2007).

\section{Meta-AnAlysis OF Movement in SDM}

The ISI Web of Knowledge (http://apps.webofknowledge.com/) was used to conduct a comprehensive search for journal articles that satisfied a query of both SDM and 'movement' as words in the article topic. While the current terminology used to refer to correlative species-environment models is converging on 'species distribution models', they have previously been referred to as 'predictive vegetation models' (Franklin, 1995), 'niche models' (Peterson et al. 2007) and 'predictive habitat distribution models' (Guisan and Zimmerman, 2000). While conceptual differences between terms do exist (e.g. modelling the actual versus potential distribution - Peterson et al. 2011), in order to correctly identify any article which could be considered under the SDM framework, all four terms were used within the search and for the purposes of this review can be considered synonymous. A variety of terms associated with organism movement were 
identified by Holyoak et al. (2008) in their quantitative study in a special issue of the Proceedings of the National Academy of Science introducing movement ecology. They identified 15 movement terms from the literature, with four key terms used in $98 \%$ of the studies surveyed; movement, migration, dispersal and gene flow. The four SDM terms and 15 movement terms identified by Holyoak et al. (2008) were used as search parameters, and while these parameters are relatively broad, I felt this was necessary in order to complete a comprehensive review. An article was deemed relevant if it referred to the movement of whole genes, progeny, organisms, populations, or species (Table 1.1). The search was conducted so that every journal article published up to and including December $31^{\text {st }} 2015$ is included, with the search considered complete as of March $9^{\text {th }}$ 2016.

The last decade has seen a surge in the incorporation of movement within SDM, with between $20-25 \%$ of all SDM studies (n.b. total number of SDM studies was calculated using the total articles returned from the four SDM terms, controlled for by the overlap observed in articles from the SDM and movement searches) published since 2010 implementing a method of movement within the analysis, or discussing but not implementing movement (Figure 1.2). In total, 595 relevant articles were identified across 180 journals (Appendix 1), illustrating just how inter-disciplinary SDM has become. I distinguished between articles that explicitly implemented movement, compared to those that only discussed movement, and it can be seen that the proportion of studies explicitly accounting for movement has increased in recent years (Figure 1.2). When movement was only discussed in the article, discussion ranged from explicitly stating that dispersal was not incorporated in the study but an acknowledgement was made stating that this likely increased uncertainty in projections (e.g. Garner et al. 2015), 
to studies that highlighted the importance of SDMs for plant migration, but made no further mention of movement factors or processes (e.g. Meineri et al. 2012).

Table 1.1: The number of articles returned from different ISI Web of Knowledge search parameters. Terms representing the correlative species-environment models are species distribution models (SDM), niche models (NM), predictive habitat distribution models (PHDM), and predictive vegetation models (PVM).

\begin{tabular}{r|cccc} 
Movement Term & SDM & NM & PHDM & PVM \\
\hline Movement & 55 & 18 & 0 & 0 \\
Migration & 103 & 35 & 0 & 0 \\
Dispersal & 266 & 106 & 0 & 2 \\
Gene Flow & 45 & 55 & 0 & 0 \\
Transport & 11 & 9 & 0 & 0 \\
Locomotor Activity & 0 & 0 & 0 & 0 \\
Gene Dispersal & 0 & 0 & 0 & 0 \\
Passage & 2 & 0 & 0 & 0 \\
Habitat Use & 41 & 12 & 1 & 0 \\
Distance Travelled & 0 & 0 & 0 & 0 \\
Site Fidelity & 2 & 0 & 0 & 0 \\
Population Connectivity & 7 & 1 & 0 & 0 \\
Interconnectivity & 1 & 0 & 0 & 0 \\
Traverse & 1 & 1 & 0 & 0 \\
Diffusion & 3 & 1 & 0 & 0
\end{tabular}




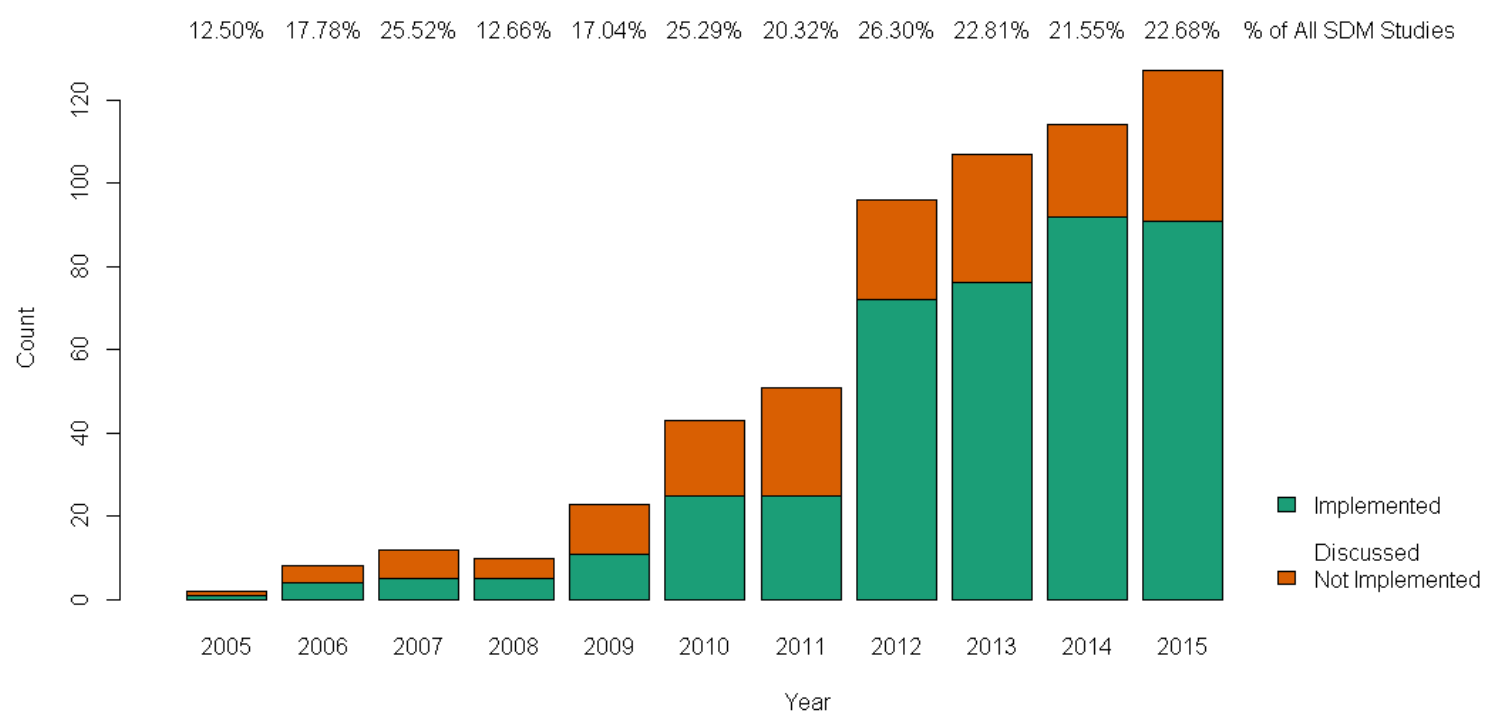

Figure 1.2: The number of SDM articles per year in which movement was implemented, or discussed but not implemented since 2005 (n.b. two SDM articles pre2005 discussed movement but were not included in the above figure).

\section{Movement TeRminology}

SDMs are used across a number of disciplines, so it is therefore vital that if movement is to be successfully incorporated into SDMs, then one must be clear in the definitions and terms used. When concepts are not well defined, it distorts communication with scientists across (and beyond) the discipline, alienates the public through ambiguous, imprecise and unstandardized answers, and it distracts from the primary aims of the research (Hall et al. 1997). SDM researchers addressing questions related to range shifts in response to the changing climate or to track the spread of invasive species have used terms such as 'dispersal limitations', 'dispersal capacities', 'migration rates', and 'spread rates' interchangeably to refer to the cumulative movement of a species or a population across a broad time scale and often across multiple generations (Miller and Holloway 2015). Definitions of movement behaviors are still strongly debated throughout the ecological disciplines (Dingle and Drake 2007), with 
terms such as 'migration' or 'dispersal' causing highly emotive responses across both the scientific and public realms. As such, it is not the purpose of this section to re-visit the debate surrounding movement definitions, but rather to provide a discussion on how movement concepts have been used in SDM, and illustrate the need for clear and concise definitions without the assumption of consensus.

Dispersal $(48.15 \%)$ was the predominant term used to describe movement when studies across all taxa and spatiotemporal scales were considered, followed by migration $(12.58 \%)$, and then movement $(8.40 \%)$, with a total of 31 general terms used to describe organism movement (Appendix 1). Only a handful of studies actually defined the terms they used. For example, Pittiglio et al. (2012) used the term transit corridor to refer to the seasonal movement of elephants in Tanzania, while Ai et al. (2012) clearly defined dispersal limitation as spatially limited dispersal in local communities. Only 46 studies (7.73\%) used a single movement term throughout the entirety of their paper. Some repetition in movement terms may have occurred due to researchers citing work which used a different term or referred to a different movement pattern; however, most of the repetition occurred due to researchers using multiple terms to refer to the same movement behavior. Moreover, in 37 studies I could not distinguish the predominant movement term used.

Dispersal and migration were often used interchangeably to refer to the same movement behavior, in particular movement in response to changing climates. Table 1.2 highlights the number of times migration or dispersal was recorded as the general term used to refer to movement in response to climate change or regular movements. Unsurprisingly, dispersal was the predominant term used for both movement patterns (as it was also the predominant term across all movement patterns), but it was used twice as much to describe tracking of the changing environment. 
The use of migration to describe the tracking of the changing environment is perhaps the most contradictory to the general consensus of what constitutes migration (e.g. the movement between two habitats on a predictable basis - Table 1.3). This movement pattern does not necessarily represent regular trips, and with the overwhelming use of dispersal to describe movement in response to climate change, this will be the term used to describe this movement pattern. Clarification of movement terms will only become more pertinent as methods continue to advance and the inclusion of multiple movement behaviors in models becomes a regular occurrence. The definitions of the main movement terms used throughout this research are outlined in Table 1.3.

Table 1.2.: The number of times dispersal or migration was used to refer to the tracking of changing environmental conditions or regular movement. The value in the brackets refers to the number of times the main terms were indistinguishable.

\begin{tabular}{lcc}
\hline & $\begin{array}{c}\text { Track Changing } \\
\text { Environment }\end{array}$ & Regular Movements \\
\hline Dispersal & $91(10)$ & $35(1)$ \\
\hline Migration & $37(10)$ & $27(1)$ \\
\hline
\end{tabular}


Table 1.3: The definitions of the main movement terms used throughout this dissertation. Terms are broadly adapted from Hansson and Åkesson (2014).

\begin{tabular}{ll}
\hline Term & Definition \\
\hline Movement & Movement is a general term that refers to the displacement of genes, \\
& progeny, individuals, populations or species that change position at \\
& any spatiotemporal period, and will be used as an overarching term \\
& with no specific inferences. \\
\hline Dispersal & Individuals, genes, progeny, populations, or species that move to \\
& reach new areas but do not return, often across a broad temporal \\
& scale and multiple generations. An assumption of 'success' is \\
& incorporated in this definition, and no differentiation is made \\
& between dispersal and colonization (and these terms can be \\
& considered interchangeably for the research investigating the \\
& tracking of the changing environment). \\
Individuals, genes, progeny, populations, or species that move \\
between two distinct habitats on a temporally predictable basis. This \\
term will mainly be used to describe seasonal migrations, which \\
describes the broad scale movement between distinct geographic \\
regions. This term will not be used to describe finer scale temporal \\
migrations (e.g. diurnal migration) as these movement patterns will \\
be incorporated within the regular movement category. \\
Individuals within a population of species that move between \\
different patches within their distribution at a finer temporal period \\
(than migration) in a reasonably predictable way. These movements \\
maintain the current distribution of the population or species, and \\
include behaviors such as foraging (searching for food), homing \\
(returning to home), bounding (marking home range limits), and \\
biotic interactions (species interactions). \\
Movements
\end{tabular}




\section{What IS Moving?}

\section{Species Groups}

SDM studies addressing movement did so for a number of different organisms, ranging from amoebas (Aguilar and Lado 2012; Aguilar et al. 2014) to elephants (Richmond et al. 2010; Pittiglio et al. 2012). However, the majority of studies which addressed movement did so for plant taxa (Figure 1.3). These outnumbered all other taxon groups across all SDM applications with the exception of projecting the current distribution (for which the majority of movement patterns were regular movements). While initially surprising that plants were recorded undertaking 'regular movements', communities of plants undergo regular turnover, and this is an important determinant in the maintenance of a species distribution. Many bird species epitomize animal movement, and it is therefore perhaps less surprising that these species constitute the second most studied group, recording equal coverage between applications of movement (with the exception of invasive spread). Plants and birds are the most studied species groups on the planet (Lomolino et al. 2006), with bird atlases collected with regularity since the 1960s (Sharrock 1976), and a disproportionately high number of available telemetry datasets for birds on data repositories such as Movebank (https://www.movebank.org/). These datasets will provide researchers with the most comprehensive data to evaluate the different movement models implemented by SDM researchers and will allow more rigorous testing and evaluation of movement models.

Of greater ecological concern are the under-studied species. Arthropods (insects, arachnids, crustaceans) constitute over half of the species recorded on the planet, but are relatively understudied compared to plants. This trend is not unique to SDM studies, with the paucity of entomologists in relation to other specialists of other taxa well documented (Lomolino et al. 2006). Arthropod species can undertake vast migration excursions (e.g. 
monarch butterflies), and should be more readily studied. Moreover, in recent years, amphibians have suffered global and substantial losses (Pounds 2001; Alford et al. 2007; Blaustein et al. 2011). Subsequently, there is a pressing need to study these species in response to global change; however, only six studies investigated the impact of global change on amphibians and incorporated a measure of dispersal alongside this. While amphibians are considered relatively poor dispersers, they have been recorded undertaking relatively long migrations between breeding and hunting locations (e.g. $3 \mathrm{~km}$ Ray et al. 2002), meaning that future projections of these species should incorporate a measure of dispersal.

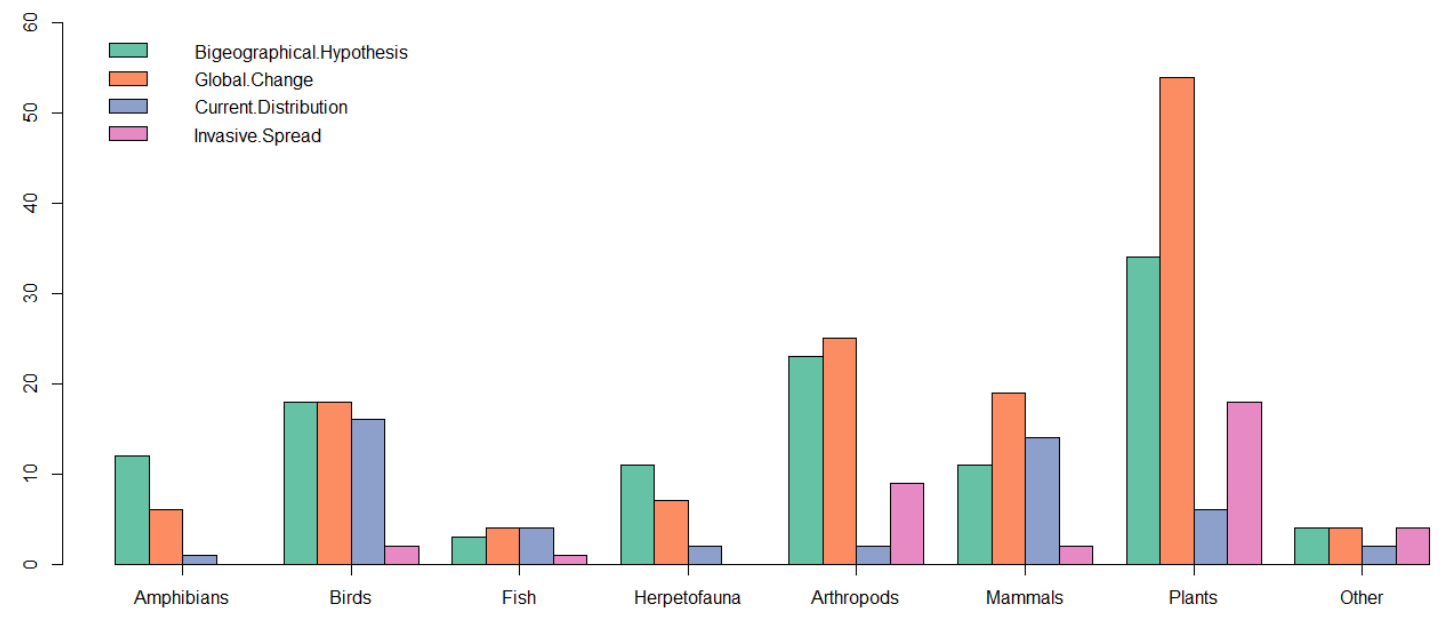

Figure 1.3: The number of SDM studies applying movement for a species group.

\section{Individuals, Populations or Species}

As noted by Levin (1992), patterns at one level of organization can often be understood as the collective behavior of aggregates of smaller units. This is pertinent when studying species distributions, as it is the movements of individuals that contain the most detail in relation to how movement and the environment interacts, but it is the emergent population- or species-level patterns that is the focus of SDM. The majority of 
studies modelled the movement of populations or species over time and many of the studies investigating biogeographic hypotheses measured the movement of genes between populations, and while this is an interesting concept, it requires detailed data that are not currently available for the majority of species.

The differences between Eulerian (population) and Lagrangian (individual) approaches to movement are important to consider, particularly as methods to model movement in SDM are increasing in complexity. Both approaches deal with populationlevel movement, but one can be considered a top-down method while the other is a bottom-up method. The Lagrangian approach involves discrete steps and segments and is useful for tracking detailed movements of individuals, while the Eulerian approach describes the expected pattern of space use by an individual or population (Smouse et al. 2010). This Lagrangian approach has been supported by ecologists and modelers (Tang and Bennett 2010) as the approach identifies population phenomena by studying the individual.

While the distribution of a species is the aggregation of all individuals, it is not possible to follow every individual of every species in the world to observe where it goes. However, with the emergence of lifetime telemetry data (Kays et al. 2015), and the potential of individual-based models to simulate the inherent relationship between movement and the environment, spatial simulation models are increasingly being used to understand animal movement, and are beginning to be observed within the SDM framework (Martinez et al. 2012; Adams et al. 2015). For example, Martinez et al. (2012) used a parameterized individual-based model which incorporated competition, facilitation and dispersal limitations to estimate a realistic rate of tree line migration under climate change in the Pyrenees. Similarly, Adams et al. (2015) used an individual- 
based cellular automata model based on dispersal constraints to model invasive spread of a non-native weed in Australia over a period of five to ten years.

Questions related to dispersal and colonization are population-level processes; however, many of the factors responsible for animal movement operate on an individual scale (Jønsson et al. 2016). While the potential to estimate population level redistributions of species using individual-based models within SDM is promising, currently only one study has addressed this beyond plants (Zurrell et al. 2012). Furthermore, all of these studies used a gridded representation of movement, which loses information as explicit idiosyncratic movements are amalgamated to a grid. These methods begin to bridge the gap between population and individual movements, as both can be incorporated in the model; however, it is the grid cells that are treated as individuals, meaning amalgamation of individuals to a gridded population is undertaken before movement is even simulated, and these models could be argued to be grid-based population models. The uptake of Lagrangian movement paths within SDM remains problematic, with the data, methods, and computing power needed to simulate broad scale spatiotemporal movements of individuals currently not sophisticated or established enough. Therefore, despite the potential to provide an extensive and detailed depiction of population redistribution based on individual movement paths, the uptake of a Lagrangian approach to incorporate movement within SDM remains a challenge.

\section{THE 'BAM' FRAMEWORK}

As outlined by the aforementioned 'BAM' framework, it is the individual and joint effects of biotic (B), abiotic (A), and movement (M) factors that determine species distributions (Figure 1.1). In particular, the incorporation of $\mathbf{M}$ within the model building framework was established so that the area the species had previously visited (M) could 
be distinguished from the area a species is absent from irrespective of $\mathbf{A}$ or $\mathbf{B}$ (Barve et al. 2011). Advancing from this, Barve et al. (2011) identified three implications of $\mathbf{M}$ within SDM: model calibration, validation, and comparison.

When calibrating an SDM, the choice of geographic extent has been found to significantly alter the projected environmental drivers (VanDerWal et al. 2009). If the geographic extent used in an SDM is beyond the dispersal capacity of the species, then the model will project the species to be absent due to the abiotic or biotic conditions, when in fact it is absent due to factors related to $\mathbf{M}$ (Barve et al. 2011). Similarly, the use of absence or pseudo-absence test data beyond $\mathbf{M}$ will most likely improve the accuracy of the projection as these areas will have a low suitability due to the lack of presence observations here. These assumptions can inflate accuracy metrics and undermine the robustness of SDM results across various applications (Barve et al. 2011). Finally, comparisons of niche similarity are an important application for many studies investigating biogeographic hypotheses (Warren et al. 2008). These comparisons require an estimate of background area, which should be considered the equivalent of $\mathbf{M}$, and with the implications related to calibration and validation can have significant implications on model inference.

Various 'BAM' scenarios exist, with different assumptions of $\mathbf{B}, \mathbf{A}$, and $\mathbf{M}$ strongly influencing all phases of model configuration (Figure 1.4). For example, Saupe et al. (2012) used virtual species to explore the implications of different BAM scenarios, explicitly pertaining to $\mathbf{A}$ and $\mathbf{M}$. They found that models that assumed that all areas were both accessible and abiotically suitable (Figure 1.4c) failed to perform better than random expectations. They cited this example as being similar to island species, whose distribution is most likely the result of dispersal limitations (M) rather than abiotic conditions (A). Similarly, $\mathbf{M}$ can be thought in terms of invasive species, which cannot 

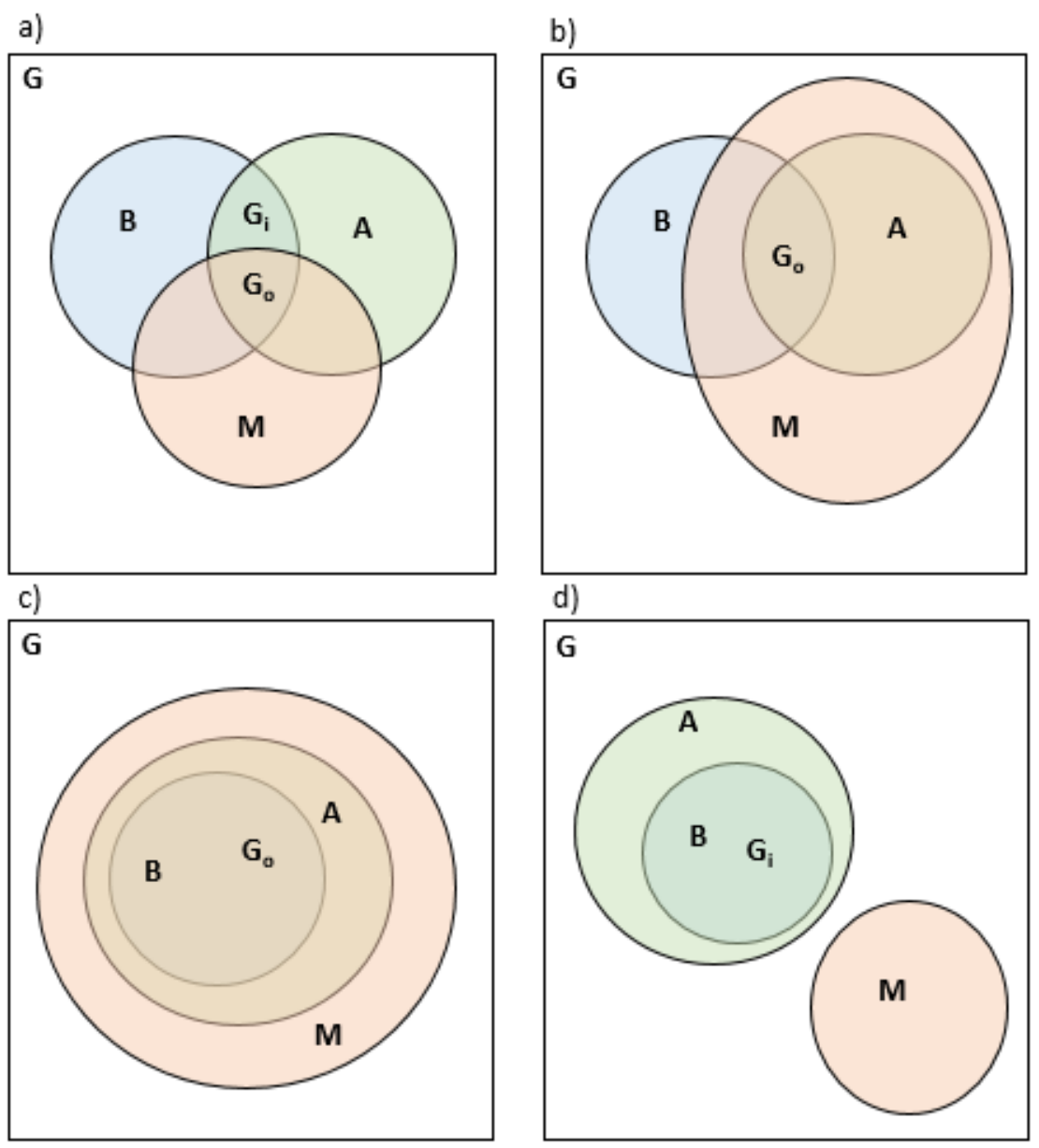

Figure 1.4: Different 'BAM' scenarios, a) represents the classic 'BAM' framework as depicted already, b) represents a situation where all the combined suitable abiotic and biotic area is accessible, so all the invadable area is null, c) represents a situation where all of $\mathbf{A}$ and $\mathbf{B}$ are accessible, and so movement does not restrict the distribution. Finally d) represents a situation where neither $\mathbf{A}$ or $\mathbf{B}$ is accessible. This could represent the potential area of an invasive species, or where potentially abiotic and biotic conditions have shifted under climate change, but the species lacks the dispersal capacity required to keep track. Modified from Peterson et al. (2011).

disperse to suitable abiotic and biotic habitats under their own ability (Figure 1.4d). These conceptualizations of $\mathbf{M}$ within the BAM framework have led to an increased understanding of model interpretation, (Barve et al. 2011; Saupe et al. 2012; Qiao et al. 2015), with a number of studies explicitly acknowledging $\mathbf{M}$ when defining modelling 
extent, pseudo-absence selection or model validation (Belaire et al. 2014; Escobar et al. 2104; Strubbe et al. 2015; McQuillan and Rice 2015; Mateo et al. 2015; Bradley et al. 2015).

In addition to the ability to access suitable environment that is represented by dispersal, $\mathbf{M}$ can also be used to describe contingent demographic factors that can facilitate dispersal or migration, such as the distribution and configuration of suitable patches required to maintain populations (Anderson, 2013; Fordham et al., 2013). This consideration of movement suitability begins to identify the dynamic nature of specieslevel dispersal, and addresses landscape issues such as fragmentation, connectivity, species-area relationships, among many of the other processes important in determining movement.

\section{APPLications OF Movement In SDM}

Generally speaking, applications of movement in SDM have consisted of either coupling the statistical model of abiotic and biotic suitability with a measure of dispersal in response to climate change or invasive spread (Franklin 2010; Miller and Holloway 2015) or as a measure of accessibility with which to select the appropriate spatial extent for model calibration, validation, and comparison (Barve et al. 2011; Saupe et al. 2012; Qiao et al. 2015). However, movement occurs across a broad range of spatiotemporal scales and as such should not be restricted to these narrow and specific movement behaviors. Following the meta-analysis, Figure 1.5 was developed as a conceptual diagram illustrating the patterns of movement that were also identified as having been studied within SDM, and the following sub-sections will discuss how these patterns of movement have been implemented, and identify the conceptual and methodological issues associated with them. 


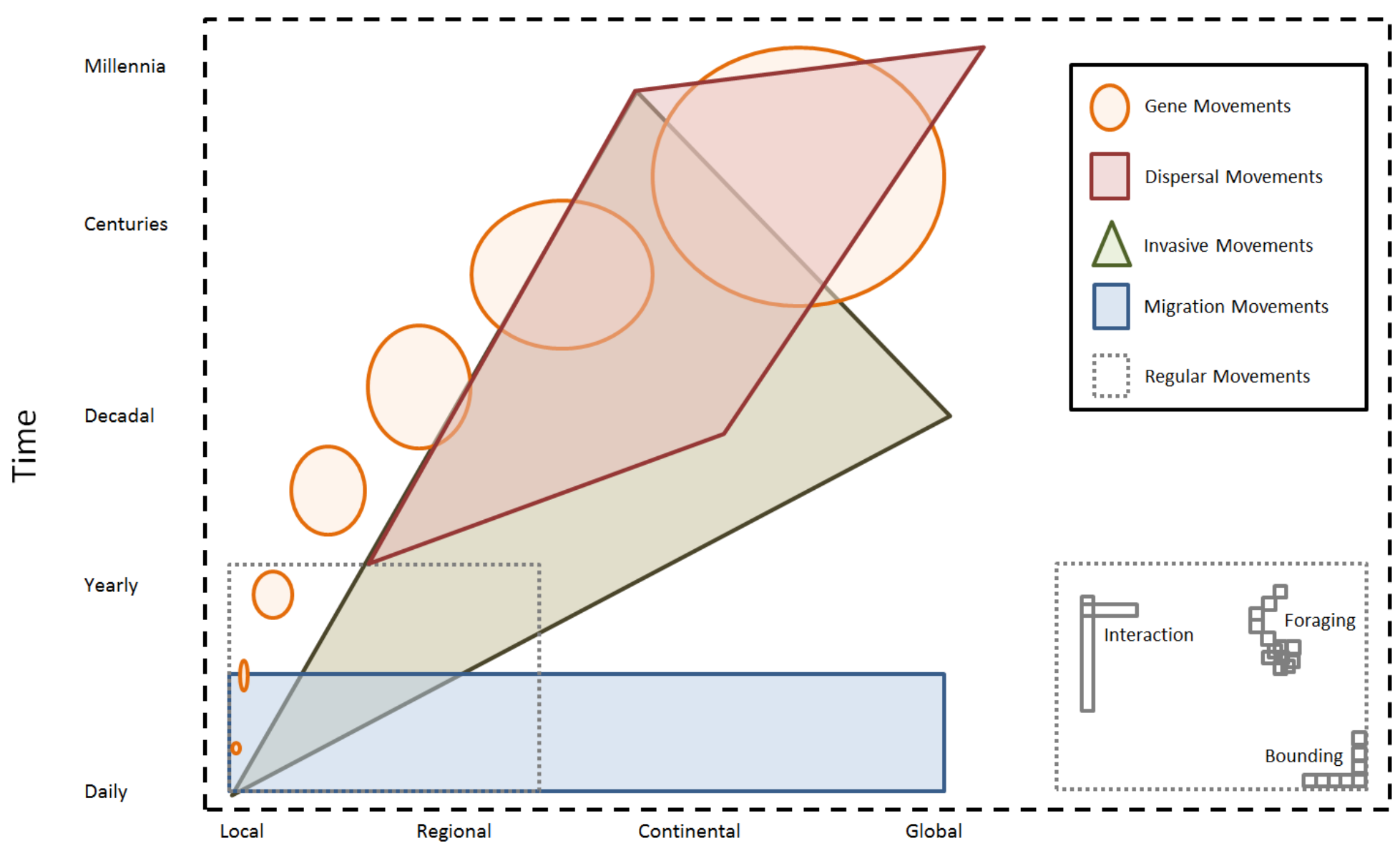

Space

Figure 1.5: The spatiotemporal scales of movement applications incorporated within SDM. 


\section{Track Changing Environmental Conditions}

Incorporating movement factors is particularly pertinent for SDMs for which the main focus is to identify changes in distributions over broad time-scales. Climate conditions and land cover are changing, and SDMs are an important tool for exploring how these changes will affect species distributions. If the new abiotic and biotic conditions are still within the range the species can tolerate, it can persist; otherwise it will need to colonize new suitable areas. Most applications involve refining a projected species distribution map to distinguish (abiotically and biotically) suitable and accessible habitat from suitable and inaccessible habitat, with accessibility measured as a function of dispersal (Miller and Holloway 2015). However, when dispersal has been implemented in SDM research, it has often taken one of two extreme approaches: either unlimited or no dispersal (e.g. Araújo et al. 2006; Lawler et al. 2006; Araújo and Luoto 2007). Unlimited dispersal assumes that there are no barriers to movement and that distance is not a limiting factor, implying that any suitable habitat which is present in the study area can become occupied. Conversely, no dispersal assumes the opposite, with dispersal not possible, and the future suitable habitat is restricted to locations that overlap with the original distribution.

The use of both unlimited dispersal and no dispersal ('all or nothing') was recorded in a quarter of the studies investigating distribution shifts under climate change. While these two approaches overly simplify movement, using both together is an improvement on studies that overlooked dispersal (and thus implicitly assumed unlimited dispersal). Furthermore, due to their extreme variation, these implementations of dispersal can be used to represent the uncertainty associated with the effects of climate change, as the actual dispersal capacity will most likely be found somewhere in the 
middle of those two predictions. A number of methods have begun to emerge in the literature, and a summary of these dispersal models is provided in Table 1.4.

When extrapolating species-environment relationships across time, there is also the assumption that the biotic and abiotic factors will remain constant for every intermediate time period. While a postulate of equilibrium is necessary for projecting the model in space and time (Guisan and Thuiller 2005), for long time periods (e.g. 100 years) it is highly unlikely that the biotic and abiotic factors will remain the same. Simulating dispersal as a multiple-step process (e.g., from $t_{1}$ to $t_{1 a}$ to $t_{1 b}$ to $t_{2}$ ) rather than the traditional one-step process (e.g., from $t_{1}$ to $t_{2}$ ), accounts for some of the inherent uncertainty related to the assumption of homogeneity of abiotic and biotic factors. For models simulating dispersal as a multiple step process, at each intermediate time-step the dispersal model is coupled with a corresponding statistical model of abiotic and biotic suitability. The next dispersal event then originates from the area predicted present based on suitability and accessibility for this time period. If the abiotic and biotic conditions alter significantly, then the number of steps used to model dispersal could greatly influence the projected distributions. For example, in a study investigating the effects of climate change on 336 Proteceae species in South Africa, Midgely et al. (2006) simulated dispersal using a fixed rate method of $4 \mathrm{~km} /$ year or $1.5 \mathrm{~km} /$ year for species dispersed by wind or rodents respectively. They found that the resulting ranges of species in 2050 were larger by approximately $10 \%$ when dispersal was simulated as a single 50 year time-step compared to when dispersal was simulated in decadal time-steps. Due to these vast differences in areal extent of future projections of geographic ranges, the uncertainty arising from modelling future distributions in multiple time-steps needs to be explored further. 
Table 1.4: The different dispersal models used to simulate movement in response to changing environmental conditions.

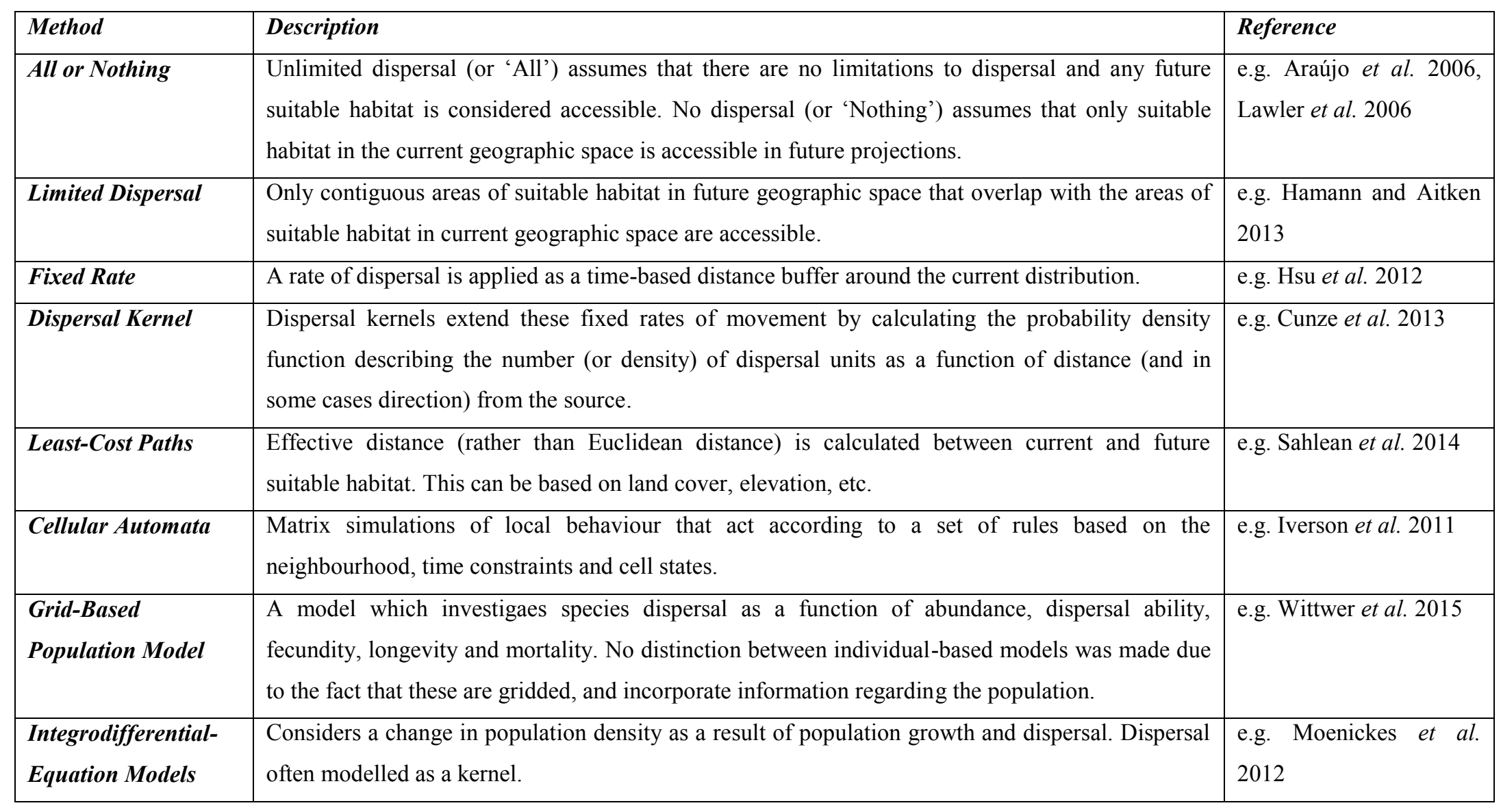


With such wide variation in the way that dispersal is represented in these models, and the differences in how dispersal can be implemented (e.g. one-step or multiple-steps), it is likely that resulting future predictions will vary markedly. Comparisons among dispersal models are rare, and have only been implemented in the assessment of 'new' models of dispersal with either an all or nothing assumption (Engler and Guisan 2009; Cunze et al. 2013). For example, Engler and Guisan (2009) found a 95\% areal difference in the projected distributions of two virtual plant species between a model incorporating dispersal and one that ignored it. Similarly, Cunze et al. (2013) found differences in projected 2080 distributions between unlimited dispersal, and a measure of 'realistic' dispersal, which took into account a combination of dispersal kernels fit for different dispersal vectors (e.g., dispersal by wind, animals, etc.). While limited comparisons have been made, there remains a need for more extensive testing across different implementations of dispersal models.

\section{Invasive Spread}

Invasive species are often characterized by excessive movements within relatively short time periods, and this movement can be a particularly confounding parameter to estimate, as species movement can be human-assisted or facilitated by long distance dispersal events. This has meant that movement of invasive spread in an SDM context has focused on post-establishment distributions (Miller and Holloway 2015). This is illustrated in Figure 1.5 as this movement pattern is depicted as expanding from a single point in space and time (e.g. the location of release or escape). Methods for incorporating post-establishment spread of invasive species in SDM studies are broadly similar to those used to investigate the impacts of climate change on distributions, meaning the research challenges and frontiers are mostly similar. 
However, if dispersal processes are not well known, incorporating fine-scale spatial autocorrelation may represent an alternative way to constrain predictions, especially in the early stages of colonization. For example, Václavík et al. (2012) used autocovariate logistic regression and spatial eigenvector modelling to incorporate spatial information into the model outputs in order to constrain projections of Sudden Oak Death in California, and found that these proxies for dispersal better predicted the presence of the pathogen compared to models calibrated only on abiotic factors. Invasive organism distributions are often driven by factors beyond environmental controls, and so a direct set of abiotic and biotic predictors may not plausible. Indirect gradients and surrogate factors therefore remain important, and studies that have used dispersal kernels (Meentemeyer et al., 2008; Ellis et al. 2010) or simple distance metrics (Václavík et al. 2010) have found that dispersal pressure was a better indicator of invasive distributions than abiotic and biotic factors alone.

\section{Seasonal Movement}

For species that move, some parts of their geographic range may temporally experience abiotic and biotic conditions beyond the tolerance of the organism. As a result, species may migrate seasonally to track the changing resources (e.g. wildebeest migrations in Africa to track seasonal rainfall patterns). However, the movement activities associated with seasonal migration may exist 'outside' what would constitute a species distribution. For example, the large swath of the Atlantic Ocean between Greenland and mainland Europe over which barnacle geese migrate is not suitable habitat. While abiotic (e.g. wind speed, direction) and biotic (e.g. increased predation) factors play important roles in determining the success of these specific migration events, there is little overlap with the movement factors as established in the 'BAM' framework. 
A simple delineation of summer and winter habitats is a common method used to account for these migrations (e.g. Martin et al. 2011), and begins to address the dynamic nature of a species distribution. A key issue associated with seasonal migrations in SDM is the observation of a species during the migration, which is subsequently in climatic conditions beyond the species tolerance. The example provided for the barnacle geese was extreme, but for many migrating species the differences in abiotic and biotic conditions across the migration corridor will be less obvious to observers. The use of these 'migration sightings' in any statistical model assumes that this species can survive in these conditions on a permanent basis, and the resultant projection will most likely over-predict the distribution (Sinclair et al. 2010).

Projecting habitat suitability along a migration corridor could address some of the issues associated with the temporal variability in predicting the distribution of migrating species distributions. For example, Hefley et al. (2015) projected the abiotic and biotic conditions for stopover habitat along the migration path of the whooping crane in Nebraska, USA, and identified areas which could support high densities of individuals during migration. This study does well to address variables along a migration pathway; however, the need to explicitly address the suitability of migration zones persists, and should be a key focus of researchers working with migratory species.

The use of temporally explicit variables removes some of the uncertainty in the seasonal distributions of species (Gscwheng et al. 2012; O’Connor et al. 2012). For example, O’Connor et al. (2012) incorporated Julien day in their generation of an SDM projecting the fall distribution of American shad and striped bass in the Hudson River Estuary, and found that it was the most important determinant of fish presence. Similarly, Gscwheng et al. (2012) matched telemetry data with abiotic conditions obtained at a monthly temporal period, and projected monthly distribution models of the Eleonora's 
falcon in Madagascar. These methods begin to identify that resource use for mobile animals is not uniform in space or time, although projections are still representative of a static species-environment relationship, albeit at a finer temporal resolution.

\section{Regular Movements}

Regular movements maintain the distribution of a population or species, and include behaviors such as foraging (searching for food), homing (returning to home), bounding (marking home range limits), and biotic interactions (e.g., predation) (Figure 1.5). These movements were distinguished as different from seasonal migration, as all of these movement behaviors also occur within the seasonal distribution of a species (e.g. foraging occurs in both breeding and wintering habitats). Explicit information of movement activity in locality data is rare, and while some studies have generated projections of suitable habitat for different activities such as nesting and foraging (Smart et al. 2012; Brambilla and Saporetti 2014; D'Elia et al. 2015), most presence data is absent of 'animal activity'. This means most projections of a species distribution inherently assume that the habitat is suitable for a multitude of activities (e.g. foraging,

nesting, etc.) which are representative of the regular movements of species. The application of activity to animal observations is routinely undertaken in animal behavior studies, identifying areas where inter-disciplinary research could bridge the gap needed within the SDM discipline.

Mobile species utilize several patches of suitable habitat within their home range, meaning species observations may be recorded in an environment which is unsuitable for them on a permanent basis. For example, many animals utilize multiple patches of suitable habitat (e.g. food sources) and due to the fragmentation of these patches, the animal has to traverse through an often inhospitable matrix (e.g. forests separated by an 
open meadow or urban area). Subsequently the aforementioned issue relating to using sightings of presence in unsuitable habitat in model calibration persists. However, as regular movements occur at a finer spatiotemporal scale, this issue could be controlled for by observing the environmental variables at a neighborhood scale (Ashcroft and Major 2013). Subsequently, for many mobile animals, the use of a focal statistic summarizing the abiotic and biotic conditions within a specified neighborhood may better represent what is available to it within its home range. For example, black grouse utilize a mixture of moorland (for mating) and woodland (for shelter) habitats, and Geary et al. (2013) identified the habitat richness of different land cover types within various neighborhood scales and incorporated this into the statistical model. The use of a focal statistic to describe the proportion of land cover types as an indicator of habitat suitability and connectivity in SDM has been found to have equal or higher predictive power than a local model, and can also help to clarify the influence of the other environmental factors that are contributing to the distribution of a mobile species (Czúcz et al. 2011; Ashcroft and Major 2013; Betts et al. 2014).

A number of methods have been proposed to incorporate a focal model, ranging from the simplistic definition of a neighborhood around a point (Arthur et al. 1996), to using distance from points (Cooper and Millspaugh 1999), weighted distances (Hjermann 2000), and topographic barriers (Matthiopoulos 2003), as covariates in the model. The methods used to identify accessible neighboring features have been found to significantly alter the importance of the environmental variables in such models (Forrester et al. 2009), meaning how researchers define 'accessible' in these models can have large implications on model projections. Currently, the methods used in SDM to describe the surrounding and available habitat do not explicitly incorporate geographic context and results are subject to uncertainty pertaining to the accessibility, connectivity, and availability of 
habitat within a neighborhood. Research needs to be directed to find models which better illustrate these issues of accessibility for mobile species. This concept is perhaps closest to Anderson's (2013) definition of 'movement suitability' which describes the contingent demographic factors that can facilitate dispersal, such as the distribution and configuration of suitable patches, and if realistic models of movement can be generated that reliability simulate these regular movements, then these could be used to identified accessibility to all features of an individual's home range, including nesting locations, boundaries, biotic interactions as well as suitable habitat.

\section{Gene Flow}

Recently, phylogeographic analysis has been integrated with SDM as a multifaceted approach to address the processes of how current distribution patterns of genes, populations and species were shaped (Carstens and Richards 2007). The ability to use SDMs to 'hindcast' species distributions has provided researchers with a means to characterize the spatial distribution of previous suitable climatic conditions for species, and these past projections have been used to determine the potential distributional and refugia areas during the late Quaternary (e.g. Veloz et al. 2012). The coupling of SDMs and phylogeographic analyses can be considered complementary, as information is provided about potential dispersal corridors from habitat suitability and genetic similarity, with both approaches used to explore and assess each other (Peterson et al. 2004). The testing of biogeographic hypotheses has recently 'exploded' in terms of its application in SDM, with 120 studies explicitly addressing a biogeographic hypothesis identified in the meta-analysis. Studies have explored several biogeographic questions such as speciation mechanisms (e.g. Raxworthy et al. 2008), niche shifts (e.g. Hill et al. 2013), and dispersal versus vicariance hypotheses (e.g. Bendiksby et al. 2014). Several 
methods have been utilized to explore gene migrations with the majority of methods identifying the most likely divergence scenario for each species using mtDNA or microsatellite data from phylogenetic analyses (e.g. MIGRATE - Inoue et al. 2015). The most likely gene migration pattern is then obtained from population-isolation analyses (Mellick et al. 2014), with the results used to identify barriers to colonization or the differences between isolated populations. For example, Chang et al. (2012) coupled SDM with an analysis of population genetic structure and demography to investigate the effect of historical climate changes on the endemic Hainan Island partridge, and concluded that its current distribution is representative of its in situ refuge and not dispersal from the mainland.

These models are more exploratory compared to the more predictive 'climate change' and 'invasive spread' movement patterns which have been incorporated more readily in SDM. However, if these methods do well to explain historical movements in response to changing climates, then they have the potential to be used to predict future changes. Recently, Razgour (2015) used a landscape genetics approach to investigate the future range shifts of the gray long-eared bat in Iberia. Implementation of the landscape genetics framework (which identified landscape variables that impede or facilitate gene flow movement) with SDM was similar to many other methods used to simulate dispersal in response to climate change, with the outputs from the landscape genetic analysis used to restrain the potentially suitable abiotic and biotic future habitat. Subsequently, there exists the possibility to combine research from both movement patterns, and use the more empirical data and analysis used in gene migration studies to predict future changes in the distribution of species, as well as identifying possible divergent events. 


\section{CONCLUSION}

Three research questions have emerged as important yet under-studied areas of incorporating movement in SDM, and are subsequently the foci of this dissertation. In particular, research will focus on two applications of movement in SDM, with three research questions forming the basis of this dissertation:

1. How does incorporating broad-scale movement (e.g. dispersal) affect SDM, especially in the context of climate change?

2. How can fine-scale regular movements be incorporated in SDM (particularly in the context of resource dynamics)?

3. How can both dispersal and regular movements be incorporated in SDM?

Dispersal in response to climate change will be studied for British birds from 1990 to 2010 in Chapter 2, and for North American flora from the Quaternary to the present in Chapter 3. By using past data to calibrate the species-environment relationships used in SDMs, a statement of accuracy for dispersal models can be made by using the present distributions of species as a test dataset. Chapter 4 will explore the inherent uncertainties associated with user-decisions when fitting the relatively new spatial ecological statistic step-selection function, directly informing Chapter 5. The inclusion of regular movements within SDM will be illustrated in Chapter 5 using a dynamic movement-resource related spatial simulation based on step-selection function for oilbirds in Venezuela. Finally, Chapter 6 will investigate how both of these movement processes that operate at distinctly different spatiotemporal scales can be integrated together into SDM. 


\section{CHAPTER 2: INCORPORATING MOVEMENT IN SPECIES DISTRIBUTION MODELS: HOW DO SIMULATIONS OF DISPERSAL AFFECT THE ACCURACY AND UNCERTAINTY OF PROJECTIONS?}

\section{INTRODUCTION}

Climate change and concomitant urbanization have led to many species shifting to higher latitudes and altitudes across Europe and North America, while many other species have simply gone extinct (Smith et al. 1993; Chen et al. 2011). In order to mitigate these potentially devastating scenarios for biodiversity, it is imperative to better understand current and future patterns of species distributions. Species distribution models (SDMs) are a powerful spatial ecological tool for studying the geographic distribution of plants, animals, and other biogeographic phenomena such as vector-borne diseases (Franklin 2009). This modelling framework provides a robust methodology for researchers and practitioners seeking to quantitatively assess the relationship between species distributions and environmental factors, and they have been used across an array of disciplines in order to test biogeographical hypotheses (Bolker et al. 2009), support nature reserve network design (Araújo et al. 2004), identify areas of rare species occurrence (Engler et al. 2004), assess species invasion risk (Medley 2010), explore the spatial scale of species-environment relationships (Miller and Hanham 2011), and assess

\footnotetext{
1 The Version of Record (VoR) of this manuscript has been published and available in the International Journal of Geographic Information Science (2016) http://www.tandfonline.com/ 10.1080/13658816.2016.1158823. Some amendments to the VoR have been made. PH was lead author on this article, developing the research question, methodology, analysis and results. As such there is no conflict for the content of this manuscript to constitute part of this dissertation.
} 
the impact of climate change on species (Wiens et al. 2011).

An increasingly important application area for SDMs is to study the effects of climate change on species distributions. The extrapolation of species-environment relationships across space or time involves several conceptual and methodological issues that introduce uncertainty, such as novel environmental combinations, species interactions, unpredictable feedbacks related to land cover change, as well as the dispersal capacity of the species. The 'BAM' framework (Figure 1.1) was developed by Soberón and Peterson (2005) and illustrates the individual and joint effects of the three most important factors in determining species distributions: biotic (B), abiotic (A), and movement (M). Biotic factors represent interactions with other species (i.e. competition, herbivory), abiotic factors represent the physiological tolerances of a species (i.e. temperature, precipitation) and movement factors refer to the processes that lead to an area being accessible to a species within a relevant timeframe (e.g., via dispersal or migration). While the importance of all three factors (abiotic, biotic and movement) is now well recognized, the majority of SDM studies have not incorporated movement, and those that do often rely on overly simplistic conceptualizations of movement (Franklin 2010; Peterson et al. 2011).

When movement has been incorporated in SDM research, it has often been to address questions related to range shifts in response to the changing climate or to track the spread of invasive species. Terms such as 'dispersal limitations', 'dispersal capacities', 'migration rates', and 'spread rates' have been used interchangeably by SDM researchers to refer to the cumulative movement of a species or a population across a 
broad time scale and often across multiple generations (Miller and Holloway 2015). It is this temporally-broad, species-level type of movement which herein is defined as dispersal.

When dispersal has been implemented in SDM research, it has often taken one of two extreme facile approaches: unlimited dispersal or no dispersal (e.g. Araújo et al. 2006; Lawler et al. 2006; Araújo and Luoto 2007). Unlimited dispersal can be considered the best case scenario representation of movement under climate change as it assumes that all future suitable habitat is accessible, while no dispersal can be considered the pessimistic worst case scenario as it assumes that only future suitable habitat that matches currently suitable habitat is accessible. Using both of these approaches together was an improvement on studies that overlooked dispersal (and thus implicitly assumed unlimited dispersal). However, these two approaches overly simplify movement and as a result of their extreme variation, can be used to represent the uncertainty associated with the effects of climate change. In reality, the actual dispersal capacity would most likely be found somewhere in the middle of those two predictions. For example, Engler and Guisan (2009) found a 95\% areal difference in the projected distributions of two virtual plant species between their model incorporating dispersal and their model that ignored it. Similarly, in a study of the spread of the non-native common waxbill (Estrilda astrild) in the Iberian Peninsula, Sullivan et al. (2012) found differences in the accuracy of predictions when a distance weight was incorporated into the SDM compared to one that assumed unlimited dispersal. Likewise, Cunze et al. (2013) found differences in the predicted 2080 distributions of European flora between unlimited dispersal and a measure 
of 'realistic' dispersal, which took into account a combination of discrete dispersal kernels fit for different dispersal vectors (e.g., dispersal by wind, animals, etc.).

Recent reviews (Franklin 2010; Miller and Holloway 2015) have identified several ways in which dispersal has been incorporated in SDM studies. Within these conceptualizations of dispersal, there are several different types of methods and subsequent variations of these methods that can be implemented, but to date, they remain untested. With such wide variation in the way that dispersal is represented in these methods, it is likely that resulting predictions will vary markedly. Limited comparisons have been made (see Engler and Guisan 2009; Sullivan et al. 2012; Cunze et al. 2013), but there is a need for more extensive testing across all implementations of dispersal models.

One of the challenges associated with comparing methods for projecting the effects of climate change on species distributions has been the lack of appropriate data with which to validate. The British Trust for Ornithology (BTO) has led the production of breeding bird atlases offering complete coverage of Great Britain at a 10km resolution in three periods spanning 40 years, in 1968-72, 1988-91, and 2008-11. These datasets can be used to test the accuracy of the dispersal models implemented in SDM research, as we can take the earliest known distribution $\left(\mathrm{t}_{1}\right)$ and project the species-environment relationships alongside dispersal to the latest time period $\left(t_{2}\right)$, and compare the results with the observed atlas data from the same period (Araújo et al. 2005). This will provide researchers with a framework to compare the differences between the dispersal models currently implemented and will subsequently allow discussions about future distributions, 
extinction rates and dispersal patterns to be made with a better understanding of the accuracy and inherent uncertainty associated with the simulation of dispersal.

\section{MethodoLOGY}

\section{Data Collection}

Distribution data for 50 breeding species were extracted from bird atlases for 1988-91 (herein 1990) (Gibbons et al. 1993) and 2008-2011 (herein 2010) (Balmer et al. 2013). Species were chosen based on a positive distribution expansion between 1990 and 2010 and visual inspection of distributions (see Balmer et al. 2013), thus reducing any limitation in discussing the accuracy of the dispersal models from using species whose distributions did not change over the course of the time period (and thus would have no variation in dispersal models). Consequently, the species span a range of attributes, such as their ubiquity, migration strategy, and whether they are native or introduced to Great Britain. Twelve birds are classified as migrant breeders, ten are classified as migrant resident breeders, 21 as resident breeders and seven as introduced breeders. The 1990 distribution was chosen instead of the 1968-1972 atlas as the earliest known distribution $\left(t_{1}\right)$ due to the lack of available land cover data pre-1990. British bird distributions vary among land cover types (Fuller et al. 2007), and land cover has changed significantly over the period (Carey et al. 2008), so must be incorporated into a dynamic model if model errors are to be ascribed to dispersal rather than a poorly constructed static model. Corresponding climate data for the 12 UK Climate Projection 2009 annual variables (Perry and Hollis 2005a, 2005b, UKCP09 (C) Crown Copyright 2009) were averaged to match the temporal range of the atlas data, as well as the five years around 2000 (see Dispersal Modelling). The climate variables included, temperature ranges, degree days, seasonal heat- and cold-waves, dry days, precipitation intensity and maximum 
precipitation. Land cover data for 1990 (Fuller et al. 1993), 2000 (Fuller et al. 2002), and 2007 (Morton et al. 2014) was obtained from the Centre for Ecology and Hydrology, with percentage coverage of arable land, coniferous forest, deciduous forest, freshwater, saltwater and urban included. Finally, a 90m Shuttle Radar Topography Mission digital elevation model from the U.S. Geologically Survey was acquired, with the mean and maximum values recorded to a $10 \mathrm{~km}$ resolution.

\section{Species Distribution Modelling}

Figure 2.1 is a conceptual diagram which highlights the main steps involved within this SDM framework. The abiotic suitability was determined by three statistical models, and accessibility across the temporal extent from 1990 to 2010 was determined with 20 dispersal models. The statistical models were used to identify abiotically suitable habitat, from which a binary surface of presence or absence was created. The dispersal models were used to identify accessible habitat, from which a binary surface of accessible or inaccessible was generated. Both of these outputs were coupled to identify habitat that was both abiotically suitable and accessible.

Although the 'BAM' framework considers biotic factors as one of the three main drivers of species distributions, interactions can vary from species to species, and are also unknown for a large number of organisms. Additionally, these interactions often occur at a spatial scale that is much finer than abiotic and movement factors (e.g. climate and dispersal - Guisan and Thuiller 2005). Habitat factors (which have been included in this research) have been suggested as a proxy for biotic interactions such as food resources (see Wisz et al. 2013); however in order to ease interpretation of the dispersal models (the main focus of this manuscript), explicit biotic interactions were not included in the analysis. For future research, biotic interactions could be incorporated as an 
environmental layer in the statistical modelling (e.g. presence or absence of a competing species, Guisan and Thuiller 2005).

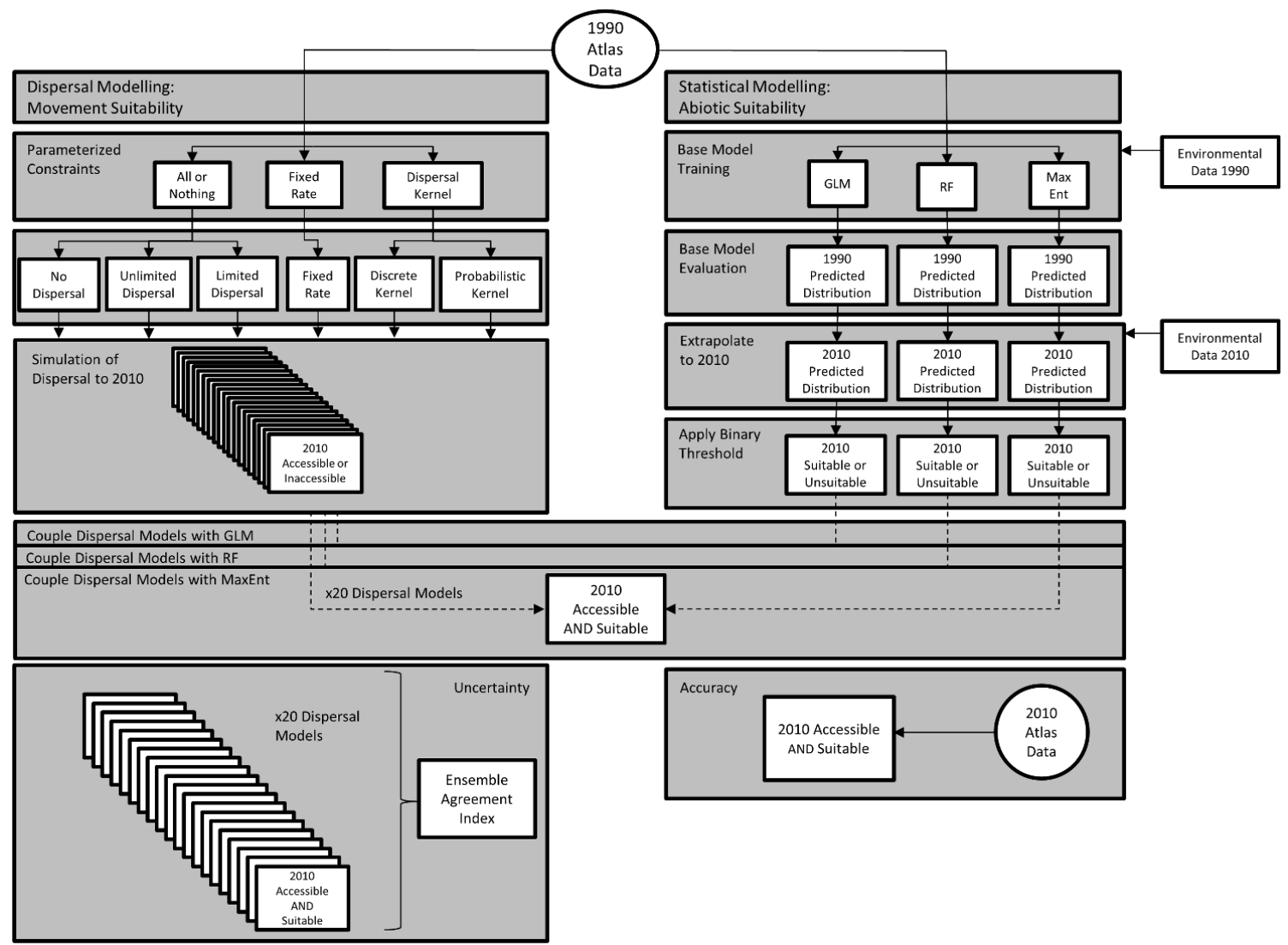

Figure 2.1: Conceptual diagram of workflow

\section{Statistical Modelling - Abiotic Suitability}

To predict the abiotically suitable habitat, three commonly used statistical methods known for generating accurate results were considered: Generalized Linear Models (GLM), Maximum Entropy (MaxEnt), and Random Forest (RF). The 1990 atlas data was fit to the 1990 climate, habitat and topography data using biomod2 (Thuiller et al. 2013). For each species, stepwise selection of environmental variables based on minimizing the Akaike Information Criterion (AIC) was used to select one final GLM, 
classification random forest was used to generate a RF model, and one MaxEnt model using default settings (with the exception of 5000 maximum iterations) was created (see Phillips and Dudik 2008). Final models will therefore differ among the 50 species, with the climate, topography and habitat variables each varying in their importance and contribution to the model.

A threshold was used to convert the suitability index into a binary measure of presence or absence. The threshold was determined based on prevalence of presences in the 1990 atlas data for GLM and RF, and the maximum training sensitivity plus specificity threshold was used for MaxEnt. The differences in threshold selection between the models were due to GLM and RF using presence/absence data in their specification and MaxEnt using presence-only data. Thresholds were selected based on guidelines recommended by similar studies (Liu et al. 2005; Hu and Jiang 2011).

Once the final statistical models had been selected, the species-environment relationships were extrapolated to the topography, climate conditions, and land cover present in 2010. Each of the three statistical methods deals with extrapolation (predicting in future, and sometimes novel or non-analogue, climate conditions) differently; GLM extrapolates by continuing the fitted trend beyond the last observation, MaxEnt acts consistently and is 'clamped' so it extrapolates in a horizontal line from the fit at the most extreme environmental value in the training data, and random forest extrapolates at a constant value from the last 'known' site (Elith and Graham 2009). These models can be considered as models with unlimited dispersal, and the dispersal models considered essentially constrain these model predictions according to different conceptualizations of dispersal. 


\section{Dispersal Modelling - Movement Suitability}

The predominant conceptualization of dispersal beyond an all or nothing approach in SDM has been to apply a parameterized constraint of dispersal to the species distribution (Franklin 2010; Miller and Holloway 2015). Within this conceptualization there are four further sub-conceptualizations: fixed rate dispersal, dispersal kernels, landscape-derived metrics and demographic models. A total of 20 dispersal models within the all or nothing, fixed rate dispersal and dispersal kernel conceptualizations were identified (Table 2.1). The 1990 atlas data was taken as the base distribution from which the 20 dispersal models were simulated. The decision to simulate dispersal from this observed distribution rather than a distribution generated by an SDM was taken so that discussion could center on the accuracy and uncertainty of the dispersal models rather than the different distributions that the three statistical methods would have generated. All dispersal models were implemented using original $\mathrm{R}$ functions written in $\mathrm{R} 3.1 .2$ ( $\mathrm{R}$ Development Core Team 2008), with the exception of the probabilistic dispersal kernel which used the kernel function in the MigClim package (Engler et al. 2013). See Appendix 2 for computational details on the dispersal models. The total 20 dispersal models and a reference code (for use in results) are provided in Table 2.1.

\section{All or Nothing}

Unlimited dispersal (UD) represents an overly optimistic scenario, and assumes that all suitable habitat in future geographic space is accessible. No dispersal (XD) is a pessimistic scenario, and assumes that only currently suitable habitat is accessible in future projections. A variant of this, limited dispersal assumes that only contiguous areas of suitable habitat in future geographic space that overlap with the areas of suitable 
habitat in current geographic space become suitable. This method can be varied by using both rook's (L1) and Queen's (L2) cases of connectivity.

Table 2.1: The 20 methods used in this study and their reference.

\begin{tabular}{l|l|} 
Method & Code \\
\hline No dispersal & XD \\
Limited dispersal (rook's) & L1 \\
Limited dispersal (Queen's) & L2 \\
Fixed rate 1 step (arithmetic mean) & FR1 \\
Fixed rate 1 step (geometric mean) & FR2 \\
Fixed rate 2 steps (arithmetic mean) & FR3 \\
Fixed rate 2 steps (geometric mean) & FR4 \\
Discrete dispersal kernel (Gaussian arithmetic) & DK1 \\
Discrete dispersal kernel (Gaussian geometric) & DK2 \\
Discrete dispersal kernel (inverse exponential arithmetic) & DK3 \\
Discrete dispersal kernel (inverse exponential geometric) & DK4 \\
Discrete dispersal kernel (Cauchy arithmetic) & DK5 \\
Discrete dispersal kernel (Cauchy geometric) & DK6 \\
Probabilistic dispersal kernel (Gaussian arithmetic) & PK1 \\
Probabilistic dispersal kernel (Gaussian geometric) & PK2 \\
Probabilistic dispersal kernel (inverse exponential arithmetic) & PK3 \\
Probabilistic dispersal kernel (inverse exponential geometric) & PK4 \\
Probabilistic dispersal kernel (Cauchy arithmetic) & PK5 \\
Probabilistic dispersal kernel (Cauchy geometric) & PK6 \\
Unlimited dispersal & UD \\
\hline
\end{tabular}




\section{Fixed Rate Dispersal}

Fixed rate (FR) involves using a rate of dispersal and applying this as a timebased distance buffer around the current distribution. This has previously been implemented using different measures of dispersal distances. For example, Gallardo et al. (2012) used the mean recorded dispersal rate of the killer shrimp (Dikerogammarus villosus) in the Rhine River to predict future distributions in the River Great Ouse in the UK, while Hsu et al. (2012) used the maximum recorded dispersal distance of tropical plants in East Asia to predict the fixed dispersal rates of forests in Taiwan. Paradis et al. (1998) published both the arithmetic and geometric means of natal dispersal distances for 75 birds in the UK from the BTO ringing scheme, and identified that dispersal distance scaled with body size with the $1 / 4$ exponent (a common feature of ecological variables, see Peters 1983). As the species between this study and Paradis et al. (1998) differed, linear regression between natal dispersal distances and average body mass of these 75 birds was run (Robinson 2005) (Appendix 3). Based on the AIC score, the model with birds grouped by breeding status was selected and used to calculate both the arithmetic and geometric means of natal dispersal distance for the birds in this study. The geometric mean restricts the impact of outliers on the mean value, which for dispersal may be representative of infrequent long-distance dispersal events and so could restrict dispersal rates.

Whether this projection is based on a fixed rate for one time step (i.e. from current $\left[\mathrm{t}_{1}\right]$ to future $\left[\mathrm{t}_{2}\right]$ ), or using a number of time steps (i.e. from current $\left[\mathrm{t}_{1}\right]$, to an intermediate period $\left[\mathrm{t}_{1.5}\right]$, to future $\left[\mathrm{t}_{2}\right]$ ) has also been shown to produce different results (Midgley et al. 2006). The multiple time step model does not assume homogenous abiotic suitability across the entire temporal extent, and couples the dispersal model with a statistical model at each time step. As this has been known to have a potential impact on 
the dispersal rates, fixed rates for one time step (1990-2010) and two time steps (19902000 and 2000-2010) were included, using corresponding climate and habitat data from that time period.

\section{Dispersal Kernels}

Dispersal kernels extend these fixed rates of movement by calculating the probability density function (PDF) describing the number of dispersal units as a function of distance from the source, with an advantage being that they can incorporate longdistance dispersal events, something that is thought to play an important role in determining broad-scale processes of population spread (Nathan et al. 2008b). Dispersal kernels can be fit with a variety of shapes. This shape is often theoretical as recorded dispersal distances for a large number of species are rare. The shape of this theoretical curve could have important consequences regarding what is considered accessible, with implementations in SDMs to date including a Gaussian distribution, an inverse exponential distribution and a fat-tailed Cauchy distribution (e.g. Conlisk et al. 2012).

The probability density function of a Gaussian distribution can be defined as:

$$
f(x, \mu, \sigma)=\frac{1}{\sigma \sqrt{2 \pi}} e^{-\frac{(x-\mu)^{2}}{2 \sigma^{2}}}
$$

where $\mu$ is the mean, $\sigma$ is the standard deviation.

The probability density function of an exponential distribution is: 


$$
f(x)=\frac{1}{\lambda} e^{-\frac{x-\mu}{\lambda}} \quad x \geq \mu ; \lambda>0
$$

where $\lambda$ is the rate parameter, which can be defined as $1 / \mu$. With the inverse of this function used for the inverse exponential PDF.

Finally, the probability density function of a Cauchy distribution is:

$$
f(x ; \mu, y)=\frac{1}{\pi y\left[1+\left(\frac{x-x_{0}}{y}\right)^{2}\right]}=\frac{1}{\pi y}\left[\frac{y^{2}}{\left(x-x_{0}\right)^{2}+y^{2}}\right]
$$

where $\mu$ is the location parameter specifying the location of the peak of the distribution, and $y$ is the scale parameter.

There are two current methodologies to implement a dispersal kernel within an SDM framework. The first is to use a discrete dispersal kernel (DK), and estimate a fixed dispersal rate according to the maximum value drawn from the kernel based on a random sample size equal to the net reproductive rate $\left(\mathrm{R}_{0}\right)$, multiplied by the temporal period $(T)$ divided by the age at which the species first breeds (A) (Clark et al. 2001; Cunze et al. 2013). $R_{0}$ was set to the maximum population density recorded for a species in an observation (Massimino et al. 2015) multiplied by the average clutch size of the species (Baillie et al. 2014), T was set to the time period (e.g. 20 years), and A was set to the breeding age for each species (Robinson and Clark 2014) (n.b. if there was no information regarding a specific species in these products, then the maximum values from another species with the same breeding status were used). The second methodology is to use a probabilistic dispersal kernel (PK) which assigns each grid a conditional probability to be colonized and generates presence based on these values. This method is currently available in the MigClim package (Engler et al. 2013). 


\section{Model Evaluation}

Model evaluation in SDM has focused on quantifying prediction accuracy as a measure of model performance or validity, such that it is known that the model has produced empirically correct predictions to a degree of accuracy that is acceptable for the models proposed application (Franklin 2009). The primary aim of this study is to assess the accuracy of SDMs projected to 2010 which are fit on 1990 environmental data and then coupled with the dispersal models. As such, the training data consists of every 1990 atlas observation, and the testing data consists of every 2010 atlas observation.

Beforehand though, it was important to evaluate the species-environment relationship fit between the 1990 atlas data and the 1990 environmental data, as without model evaluation, any judgement of the performance of the dispersal models when coupled with the 2010 statistical model cannot be excluded from the possibility of poor model fit in general. The final SDMs were built with every observation; however, alternative strategies were explored (e.g. partitioning the data into $80 \%$ training and $20 \%$ testing, Fielding and Bell 1997) and it was found that the accuracy was equally high if independent test data or resubstituted test data were used (data not shown). The species used in this study were deemed to have sufficiently high accuracy scores for data trained and tested on the 1990 observations (e.g. sensitivity of 0.7 or higher).

Similarly, it is important to assess the performance of the 2010 base models, which are also the models of unlimited dispersal. As unlimited dispersal is the most optimistic scenario, any dispersal models coupled with this prediction will either result in the same number of predicted presences or fewer. If the base model contains only a few presences, then the dispersal models will fail to restrain the projections and the results will suggest that the models have little impact on the results when in fact this would be based on a poorly specified statistical model. Finally, the evaluation of the performance 
of the 2010 statistical models coupled with all the of the dispersal models is desired. All three stages can be evaluated using threshold-dependent accuracy metrics, while threshold-independent metrics will also be used to assess the 1990 and 2010 base models.

Table 2.2: Threshold-dependent accuracy measures for species presence/absence models. $\mathrm{TP}=$ True Positive, $\mathrm{TN}=$ True Negative, $\mathrm{FP}=$ False Positive, $\mathrm{FN}=$ False Negative.

\begin{tabular}{|c|c|}
\hline Metric & Equation \\
\hline Sensitivity & $\mathrm{TP} /(\mathrm{TP}+\mathrm{FN})$ \\
\hline Specificity & $\mathrm{TN} /(\mathrm{TN}+\mathrm{FP})$ \\
\hline Proportion Correctly & $(\mathrm{TP}+\mathrm{TN}) / \mathrm{n}$ \\
\hline \multicolumn{2}{|l|}{ Classified (PCC) } \\
\hline True Skill Statistic (TSS) & (Sensitivity + Specificity) -1 \\
\hline Kappa & {$[(\mathrm{TP}+\mathrm{TN})-(\underline{((\mathrm{TP}+\mathrm{FN})(\mathrm{TP}+\mathrm{FP})+(\mathrm{FP}+\mathrm{TN})(\mathrm{FN}+\mathrm{TN}))})]$} \\
\hline & $\frac{\mathrm{n}}{\left[n-\left(\frac{(\mathrm{TP}+\mathrm{FN})(\mathrm{TP}+\mathrm{FP})+(\mathrm{FP}+\mathrm{TN})(\mathrm{FN}+\mathrm{TN})}{\mathrm{n}}\right)\right]}$ \\
\hline
\end{tabular}

The conversion of the probability surface into a binary (presence/absence) product allows the model to be evaluated by a two-by-two contingency table which crosstabulates true and false positives (presences) and true and false negatives (absences) (Franklin 2009). Table 2.2 identifies the five threshold-dependent metrics used to evaluate the models. Threshold-independent metrics have also been used in recent studies due to the potential uncertainty and subjectivity related to the selection of thresholds (see Liu et al. 2005 for a discussion). The receiver operating characteristic (ROC) plot, the most commonly used threshold-independent metric, is a graph of the false-positive error rate on the $\mathrm{x}$-axis plotted against the true positive rate on the y-axis, with the area under 
the curve (AUC) calculated by summing the area under the ROC curve. However, as the process of coupling the abiotic suitability models with the dispersal models requires the use of a presence/absence product, AUC can only be used to evaluate the original 1990 SDM and the 2010 SDM with unlimited dispersal.

\section{Quantification of Uncertainty}

In order to effectively interpret the uncertainty associated with the 20 dispersal models, the Ensemble Agreement Index (EAI) was developed which ranks full agreement across every observation as 1 , and no agreement across every observation as 0 :

$$
E A I=\frac{\left(\frac{E-\min (E)}{\max (E)-\min (E)}\right)}{N}
$$

where $N$ is the total number of presence observations in the output, and $E$ is defined as:

$$
E=\left(\sum \frac{\mathrm{e}}{m}\right)
$$

where $e$ is the ensemble score for one presence observation, defined as the number of coupled statistical and dispersal models that predicted occupancy at a particular location, and $m$ is the total number of dispersal models used to generate the ensemble. The use of descriptive statistics can be used with categorical variables in order quantify the shape of the distribution of outcomes and be used as a measure of confidence to assess the index (Hill et al. 2005). A measure of kurtosis was calculated alongside the Ensemble Agreement Index, with a leptokurtic distribution suggesting a peaked distribution (or high agreement among dispersal models), and a platykurtic distribution suggesting a flat distribution (or low agreement among dispersal models). A kurtosis 
value twice the standard error was considered significantly different from a normal distribution, and recorded as such.

\section{RESUlts}

Table 2.3 shows the mean and standard deviation of the accuracy scores for the three statistical methods fit on the 1990 environmental data and projected using the 2010 environmental data, with the assumption of unlimited dispersal across the 50 species. No single statistical method reported consistently higher accuracy metrics when averaged across the 50 species. It was found that GLM and RF reported high accuracy scores for the 2010 model for the more widespread species in 1990; however these methods seriously under-predicted the 2010 distributions for many of the species with sparser ranges in 1990 (data not shown). While under-prediction did occur for MaxEnt, it was only an issue for a few species, as is evident by the higher average sensitivity score (Table 2.3).

This is further supported by Figure 2.2, which illustrates the range of sensitivity scores for the 20 dispersal models and the three statistical methods for the 50 species (separated based on UK breeding status). Boxplots are ordered so that XD is no dispersal (or worst case scenario) and UD is unlimited dispersal (or best case scenario), with intermediate conceptualizations of dispersal in between. (n.b. the six iterations of the discrete dispersal kernel are consolidated under DK, as there were no visible differences in sensitivity scores, but that these methods are treated separately for statistical analysis). 
Table 2.3: $\quad$ Mean and standard deviation (SD) of accuracy metric scores for the three statistical methods fitted on the 1990 environmental data and projected using the 2010 environmental data, with the assumption of unlimited dispersal across the 50 species. Abbreviations stand for Proportion Correctly Classified (PCC), True Skill Statistic (TSS) and Area Under the Curve (AUC).

\begin{tabular}{l|llllll}
\multicolumn{1}{c}{} & Sensitivity & Specificity & PCC Mean & Kappa & TSS Mean & AUC \\
\hline \multirow{2}{*}{$G L M$} & Mean (SD) & Mean (SD) & (SD) & Mean (SD) & (SD) & Mean (SD) \\
\hline \multirow{4}{*}{ MaxEnt } & 0.532 & 0.855 & 0.738 & 0.325 & 0.386 & 0.693 \\
& $(0.209)$ & $(0.108)$ & $(0.121)$ & $(0.180)$ & $(0.180)$ & $(0.090)$ \\
$R F$ & 0.601 & 0.828 & 0.732 & 0.343 & 0.286 & 0.714 \\
& $(0.208)$ & $(0.137)$ & $(0.115)$ & $(0.168)$ & $(0.168)$ & $(0.084)$ \\
& 0.563 & 0.857 & 0.803 & 0.403 & 0.420 & 0.710 \\
& $(0.251)$ & $(0.142)$ & $(0.101)$ & $(0.185)$ & $(0,197)$ & $(0.098)$
\end{tabular}

The largest variability in sensitivity scores occurs for the MaxEnt models, then to a lesser extent GLM, but there is minimal variation in the RF models. The largest difference in accuracy measures occurs for the MaxEnt models of the introduced breeders. The two most extreme dispersal models (XD and UD) bound the other methods, which vary quite substantially in their sensitivity scores. The two limited dispersal models (L1 and L2) result in higher sensitivity than many of the dispersal models with parameterized constraints. Differences were also observed in the overall sensitivity score for the probabilistic dispersal kernels (PK) for birds with different breeding statuses.

No single dispersal model recorded the highest accuracy for all 50 species and five accuracy metrics (Figure 2.3). No dispersal (XD) achieved the most 'successes', obtaining the highest accuracy scores in 113 scenarios. In part this is because XD recorded the highest specificity for all 50 species, with only PK1 recording an equal 
specificity score for the great skua (Stercorarius skua). While XD resulted in the highest number of accuracy metrics, it achieved the fewest highest sensitivity scores. Several dispersal models recorded the highest sensitivity score for all 50 species (UD, PK3, DK1, DK3, DK5, DK6), and this was the most variable accuracy metric among dispersal models.

In addition to differences in accuracy, these methods also resulted in quite variable areal extents of predicted presence. Table 2.4 shows a pairwise comparison of the performance of the different dispersal models when coupled with MaxEnt, which shows the difference in area $\left(\mathrm{km}^{2}\right)$ predicted present among each of the 20 dispersal models averaged across the 50 species. Whether this areal change was also accompanied by a significant change in the true skill statistic (TSS) is also highlighted. TSS was chosen as the accuracy metric, as it rewards both prediction for correctly predicted presences and absences. Paired sampled t-tests were used to identify significant differences in accuracy among the methods $(\alpha<0.05)$. The use of no dispersal (XD) resulted in a decrease in predicted area compared to every other method, as well as a significant decrease in TSS compared to all the other methods, with the difference in area varying between values of $29,708 \mathrm{~km}^{2}$ for FR4 and $36,640 \mathrm{~km}^{2}$ for UD. Unlimited dispersal (UD) differed in TSS when compared to only six methods, including four of the probabilistic dispersal kernels; however, the limited dispersal models (L1 and L2) and one step fixed rate dispersal models (FR1 and FR2) did not differ significantly. 

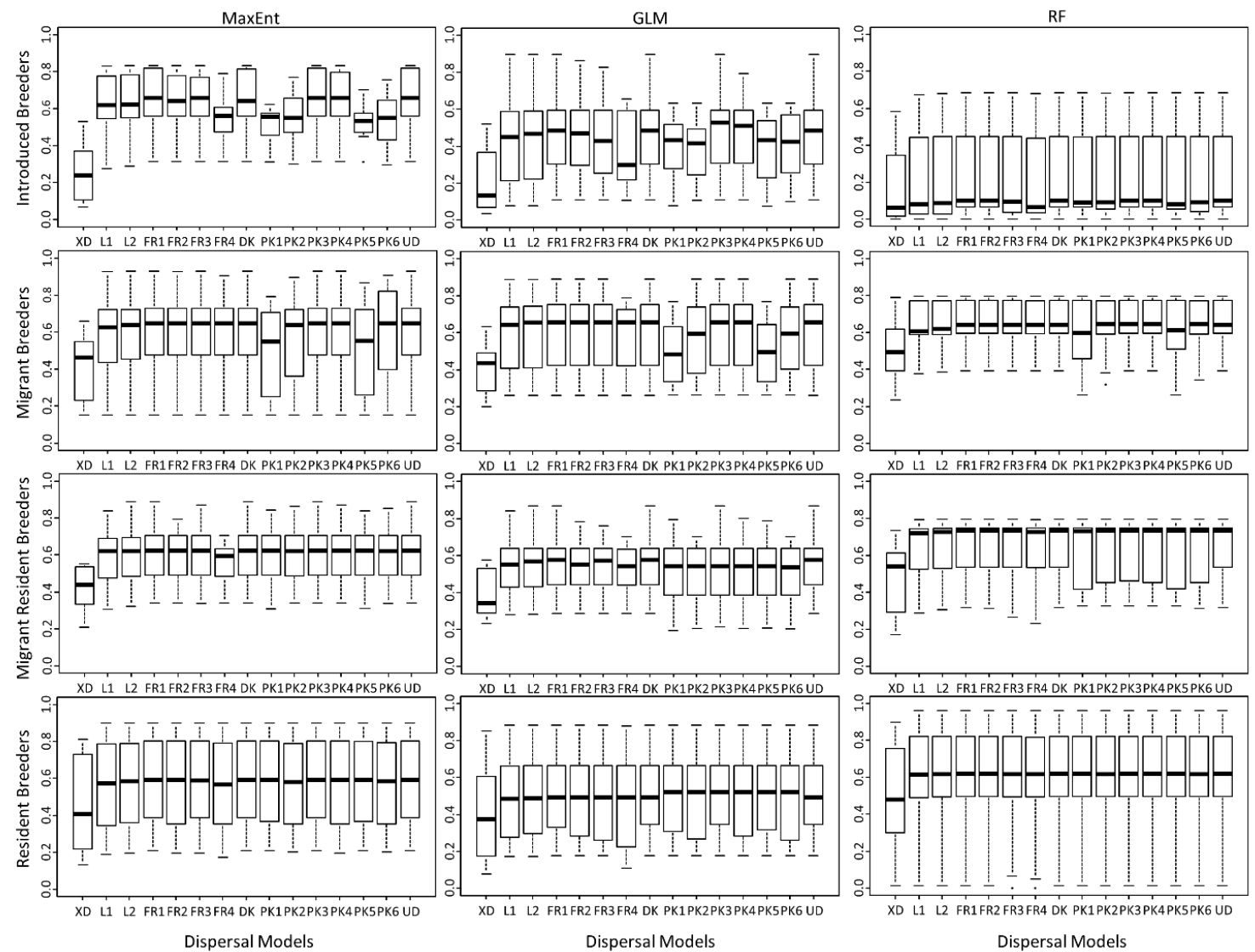

Figure 2.2: Sensitivity scores for the 20 dispersal models for the three statistical methods and four breeding groups. $\mathrm{XD}$ is no dispersal, $\mathrm{L}$ is limited dispersal ( $1=$ rook's, $2=$ Queen's $)$, FR is fixed rate $(1=1$ step arithmetic, $2=1$ step geometric, $3=2$ step arithmetic, $4=2$ step geometric), DK is discrete kernel, PK is probabilistic kernel, UD is unlimited dispersal. For Kernels, 1 $=$ Gaussian PDF arithmetic, $2=$ Gaussian PDF geometric, $3=$ inverse exponential PDF arithmetic, $4=$ inverse exponential PDF geometric, $5=$ Cauchy PDF arithmetic, $6=$ Cauchy PDF geometric (n.b. DK1 to DK6 are consolidated under DK due to no visible variation). 


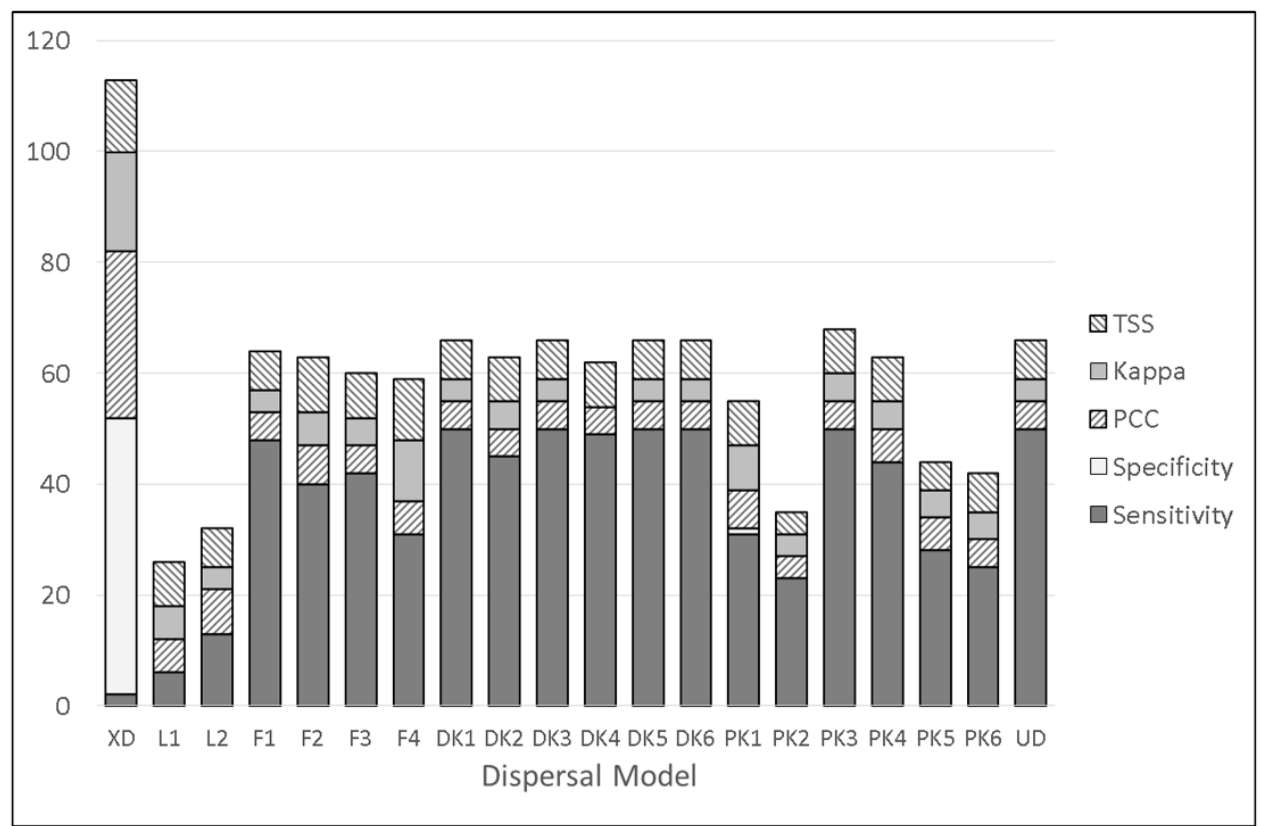

Figure 2.3: Number of times each dispersal model recorded the highest value for a particular accuracy metric for each individual species. Across the 50 species and five accuracy metrics, the highest possible value could have been 250 .

Differences in performance of methods using geometric and arithmetic means were minimal, suggesting that the restriction of the geometric average did not constrain the models as expected. However, the one-step fixed rate methods (FR1 and FR2) resulted in an increase in accuracy compared to the geometric two-step method (FR4), but not the arithmetic two-step method (FR3). There were no significant differences between the different iterations of the discrete dispersal kernels. The two discrete kernels with Cauchy PDFs (DK5 and DK6) and the inverse exponential using arithmetic values (DK3) were the only methods which did not result in any change in predicted area compared to each other or unlimited dispersal (UD) for all 50 species. 
Figure 2.4 shows the ensemble maps for nine species varying in breeding status and model uncertainty. The ensemble scores for the three medium uncertainty species (Figures 2.4(a-c)) and the three introduced species (Figures 2.4(d-f)) all identify a core area of low uncertainty (approximate score of 16-20), but then as distance from this core increases, the ensemble score decreases. Likewise, the more fragmented a predicted distribution, the greater variability and uncertainty of accessibility increases.

Table 2.5 presents the Ensemble Agreement Index (EAI) developed in order to quantify the uncertainty related to the dispersal models for all 50 species. The EAI score ranges from 0 (high uncertainty, low agreement) to 1 (low uncertainty, high agreement). When all 20 dispersal models are incorporated in the EAI, the scores range from 0.740 for the Dartford warbler (a resident breeder) to 0.999 for the Blackcap (a migrant breeder). Several species have EAI scores over 0.990, suggesting a very high number of observations with full agreement. When the six discrete kernel methods were removed, the species with medium to low uncertainty dropped to medium uncertainty (e.g. Dartford warbler and wood duck). However, this did not alter the EAI scores of the low uncertainty species (e.g. blackcap and green woodpecker), showing that this method proves robust in identifying low uncertainty among methods. 


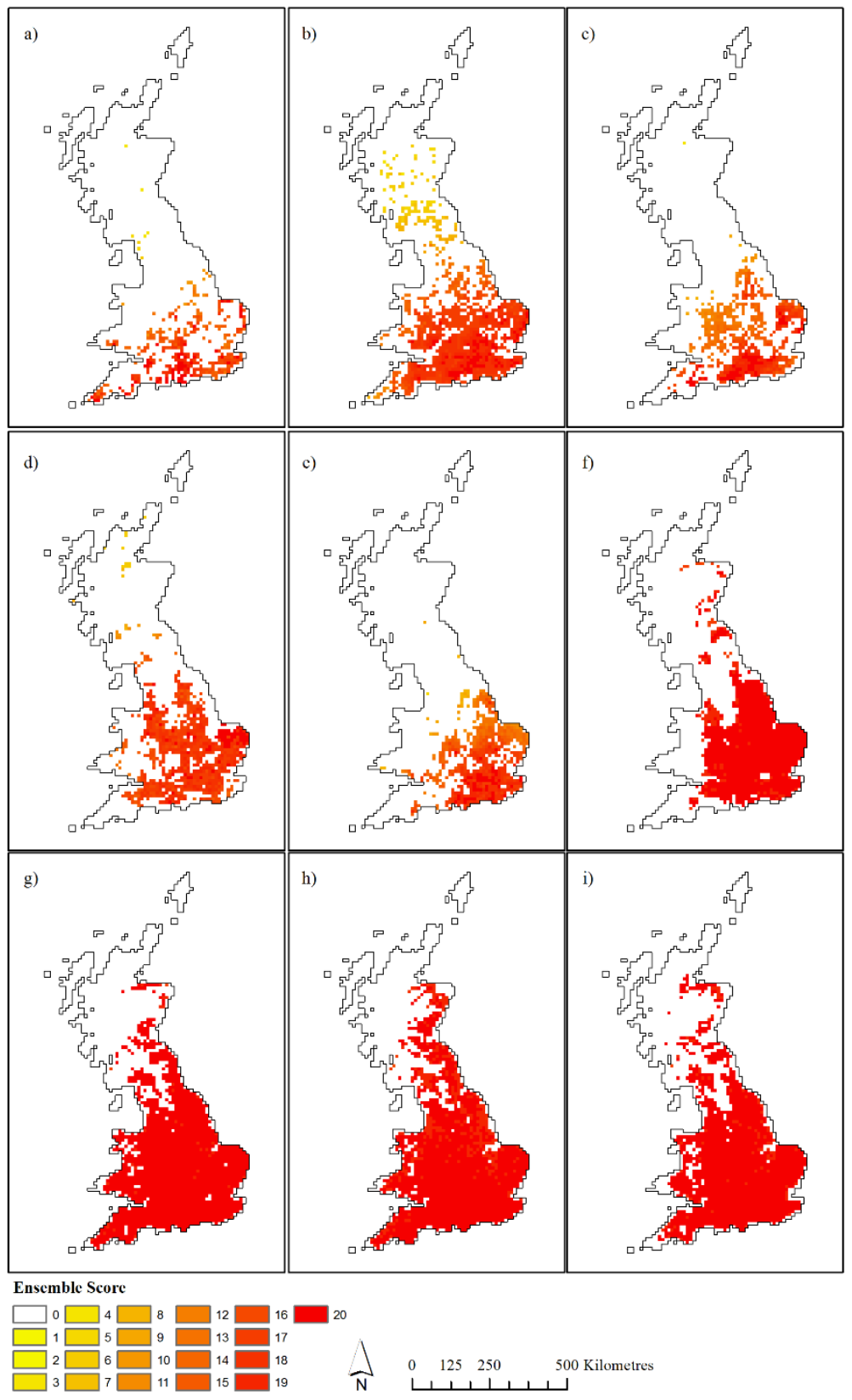

Figure 2.4: Ensemble maps of predicted presence based on environmental suitability (using MaxEnt) and the 20 dispersal models for 2010 for $\mathrm{a}-\mathrm{c}$ ) three species with high uncertainty (Cetti's Warbler, Firecrest, Woodlark), d-f) three introduced species (Egyptian Goose, Ring-necked Parakeet, Redlegged Partridge) and $\mathrm{g}-\mathrm{i}$ ) three with high modelling certainty (Blackcap, Green Woodpecker, Great Spotted Woodpecker). 
Table 2.4: Pairwise comparison of the performance of the 20 dispersal methods. The value represents the average change in area $\left(\mathrm{km}^{2}\right)$ predicted present averaged for the 50 species between the method in the row compared to the method in the column. Green represents a significant increase in accuracy (True Skill Statistic - TSS) and red represents a significant decrease in accuracy (TSS) between the methods. Significance of $\alpha=0.05$ calculated using a paired sample t-Test. XD is no dispersal, $\mathrm{L}$ is limited dispersal $(1=$ rook's, $2=$ Queen's $)$, FR is fixed rate $(1=1$ step arithmetic, $2=1$ step geometric, $3=2$ step arithmetic, $4=2$ step geometric), DK is discrete kernel, PK is probabilistic kernel, UD is unlimited dispersal. For Kernels, 1 $=$ Gaussian Probability Density Function (PDF) arithmetic, $2=$ Gaussian PDF geometric, $3=$ inverse exponential PDF arithmetic, 4 = inverse exponential PDF geometric, $5=$ Cauchy PDF arithmetic, $6=$ Cauchy PDF geometric.

\begin{tabular}{|c|c|c|c|c|c|c|c|c|c|c|c|c|c|c|c|c|c|c|c|c|}
\hline & XD & L1 & L2 & FR1 & FR2 & FR3 & FR4 & DK1 & DK2 & DK3 & DK4 & DK5 & DK6 & PK1 & PK2 & PK3 & PK4 & PK5 & PK6 & UD \\
\hline XD & & & & & & & & & & & & & & & & & & & & \\
\hline L1 & 31848 & & & & & & & & & & & & & & & & & & & \\
\hline L2 & 34010 & 2162 & & & & & & & & & & & & & & & & & & \\
\hline FR1 & 36276 & 4428 & 2266 & & & & & & & & & & & & & & & & & \\
\hline FR2 & 33588 & 1740 & -422 & -2688 & & & & & & & & & & & & & & & & \\
\hline FR3 & 35616 & 3768 & 1606 & -660 & 2028 & & & & & & & & & & & & & & & \\
\hline FR4 & 29708 & -2140 & -4302 & -6568 & -3880 & -5908 & & & & & & & & & & & & & & \\
\hline DK1 & 36382 & 4534 & 2372 & 106 & 2794 & 766 & 6674 & & & & & & & & & & & & & \\
\hline DK2 & 35698 & 3850 & 1688 & -578 & 2110 & 82 & 5990 & -684 & & & & & & & & & & & & \\
\hline DK3 & 36640 & 4792 & 2630 & 364 & 3052 & 1024 & 6932 & 258 & 942 & & & & & & & & & & & \\
\hline DK4 & 36322 & 4474 & 2312 & 46 & 2734 & 706 & 6614 & -60 & 624 & -318 & & & & & & & & & & \\
\hline DK5 & 36640 & 4792 & 2630 & 364 & 3052 & 1024 & 6932 & 258 & 942 & 0 & 318 & & & & & & & & & \\
\hline DK6 & 36640 & 4792 & 2630 & 364 & 3052 & 1024 & 6932 & 258 & 942 & 0 & 318 & 0 & & & & & & & & \\
\hline PK1 & 30382 & -1466 & -3628 & -5894 & -3206 & -5234 & 674 & -6000 & -5316 & -6258 & -5940 & -6258 & -6258 & & & & & & & \\
\hline PK2 & 31104 & -744 & -2906 & -5172 & -2484 & -4512 & 1396 & -5278 & -4594 & -5536 & -5218 & -5536 & -5536 & 722 & & & & & & \\
\hline PK3 & 36552 & 4704 & 2542 & 276 & 2964 & 936 & 6844 & 170 & 854 & -88 & 230 & -88 & -88 & 6170 & 5448 & & & & & \\
\hline PK4 & 35316 & 3468 & 1306 & -960 & 1728 & -300 & 5608 & -1066 & -382 & -1324 & -1006 & -1324 & -1324 & 4934 & 4212 & -1236 & & & & \\
\hline PK5 & 31016 & -832 & -2994 & -5260 & -2572 & -4600 & 1308 & -5366 & -4682 & -5624 & -5306 & -5624 & -5624 & 634 & -88 & -5536 & -4300 & & & \\
\hline PK6 & 31452 & -396 & -2558 & -4824 & -2136 & -4164 & 1744 & -4930 & -4246 & -5188 & -4870 & -5188 & -5188 & 1070 & 348 & -5100 & -3864 & 436 & & \\
\hline UD & 36640 & 4792 & 2630 & 364 & 3052 & 1024 & 6932 & 258 & 942 & 0 & 318 & 0 & 0 & 6258 & 5536 & 88 & 1324 & 5624 & 5188 & \\
\hline
\end{tabular}


The use of kurtosis added confidence to the EAI as it illustrated whether all the observations were in one category or spread across several. Many of the species have leptokurtic distributions and are accompanied by a high EAI (Table 2.5), suggesting that there is high agreement among all of the methods. Some species (Cetti's warbler, firecrest) have a relatively low EAI score, but leptokurtic dispersion. This suggests that many of the methods do in fact agree, but the distribution of observations is most likely grouped into two peaks, one containing the expansive methods of dispersal, and another containing the more restrictive methods. Platykurtic dispersion suggests a flatter than usual distribution, and these are found for the more sparse species (e.g. Dartford warbler) and a surprisingly large number of raptors (e.g. osprey, goshawk, and hobby).

Table 2.5: Ensemble Agreement Index (EAI) scores for the 50 species. A value of 1 indicates all 20 methods made the same predictions for all locations. - $1 \mathrm{k}$ represents a significant lepokurtic distribution. - pk represents a significant platykurtic distribution. A kurtosis value twice the standard error was considered significantly different from a normal distribution.

\begin{tabular}{|c|c|c|}
\hline Species & $\begin{array}{l}\text { EAI with Discrete } \\
\text { Kernels }\end{array}$ & $\begin{array}{c}\text { EAI without } \\
\text { Discrete Kernels }\end{array}$ \\
\hline Blackcap (Sylvia atricapilla) & $0.999^{-1 p}$ & $0.998^{-1 \mathrm{p}}$ \\
\hline Great Spotted Woodpecker (Dendrocopos major) & $0.997^{-1 \mathrm{p}}$ & $0.995^{-1 p}$ \\
\hline Grey Heron $($ Ardea cinerea $)$ & $0.996^{-1 p}$ & $0.993^{-1 p}$ \\
\hline Grey Wagtail (Motacilla cinerea) & $0.993^{-1 p}$ & $0.990^{-1 p}$ \\
\hline Common Buzzard (Buteo buteo) & $0.992^{-1 p}$ & $0.989^{-1 p}$ \\
\hline Oystercatcher (Haematopus ostralegus) & $0.992^{-1 p}$ & $0.989^{-1 p}$ \\
\hline Red-legged Partridge (Alectoris rufa) & $0.992^{-1 p}$ & $0.988^{-\mathrm{pk}}$ \\
\hline Canada Goose (Branta canadensis) & $0.991^{-1 p}$ & $0.987^{-1 p}$ \\
\hline Green Woodpecker (Picus viridis) & $0.990^{-1 p}$ & $0.986^{-1 p}$ \\
\hline Nuthatch (Sitta europea) & $0.990^{-1 p}$ & $0.986^{-1 p}$ \\
\hline Raven (Corvus corax) & $0.990^{-1 p}$ & $0.985^{-1 p}$ \\
\hline Kingfisher (Alcedo atthis) & $0.988^{-1 p}$ & $0.982^{-\mathrm{lp}}$ \\
\hline Tree Sparrow (Passer montanus) & $0.988^{-1 p}$ & $0.982^{-1 p}$ \\
\hline
\end{tabular}


Table 2.5 (continued):

\begin{tabular}{|c|c|c|}
\hline Sand Martin (Riparia riparia) & $0.985^{-\mathrm{pk}}$ & $0.978^{-\mathrm{pk}}$ \\
\hline Herring Gull (Larus argentatus) & $0.985^{-\mathrm{lp}}$ & $0.978^{-1 p}$ \\
\hline Lesser Black-backed Gull (Larus fuscus) & $0.985^{-\mathrm{lp}}$ & $0.978^{-\mathrm{lp}}$ \\
\hline Siskin (Spinus spinus) & $0.985^{-1 p}$ & $0.977^{-1 p}$ \\
\hline Barn Owl (Tyto alba) & $0.982^{-1 p}$ & $0.973^{-1 p}$ \\
\hline Peregrine Falcon (Falco peregrinus) & $0.982^{-1 p}$ & $0.973^{-1 p}$ \\
\hline Reed Warbler (Acrocephalus scirpaceus) & $0.982^{-1 p}$ & $0.974^{-\mathrm{pk}}$ \\
\hline Common Eider (Somateria mollissima) & $0.981^{-1 p}$ & $0.972^{-1 p}$ \\
\hline Goosander (Mergus merganser) & $0.981^{-1 p}$ & $0.973^{-1 p}$ \\
\hline Stonechat (Saxicola rubicola) & $0.979^{-1 p}$ & $0.970^{-1 p}$ \\
\hline Little Grebe (Tachybaptus ruficollis) & $0.978^{-1 p}$ & $0.967^{-1 p}$ \\
\hline Shelduck (Tadorna tadorna) & $0.977^{-1 p}$ & $0.967^{-1 p}$ \\
\hline Quail (Coturnis coturnix) & $0.974^{-1 p}$ & $0.962^{-1 p}$ \\
\hline Cormorant (Phalacrocora carbo) & $0.973^{-1 p}$ & $0.961^{-1 p}$ \\
\hline Grasshopper Warbler (Locustella naevia) & $0.971^{-1 p}$ & $0.958^{-1 p}$ \\
\hline Common Tern (Sterna hirunda) & $0.970^{-1 p}$ & $0.955^{-1 p}$ \\
\hline Greylag Goose (Anser anser) & $0.966^{-1 p}$ & $0.951^{-1 p}$ \\
\hline Long-eared Owl (Asio otus) & $0.951^{-\mathrm{lp}}$ & $0.928^{-1 p}$ \\
\hline Hobby (Falco subbuteo) & $0.950^{-\mathrm{pk}}$ & $0.926^{-\mathrm{pk}}$ \\
\hline Water Rail (Rallus aquaticus) & $0.946^{-1 p}$ & $0.921^{-\mathrm{lp}}$ \\
\hline Gadwall (Anas strepera) & $0.944^{-1 p}$ & $0.917^{-\mathrm{pk}}$ \\
\hline Mandarin Duck (Aix galericulata) & $0.938^{-1 p}$ & $0.910^{-1 p}$ \\
\hline Goshawk (Accipter gentilis) & $0.937^{-1 p}$ & $0.909^{-\mathrm{pk}}$ \\
\hline Wigeon (Anas penolope) & $0.935^{-\mathrm{lp}}$ & $0.905^{-\mathrm{lp}}$ \\
\hline Little Ringed Plover (Charadrius dubius) & $0.927^{-1 p}$ & $0.894^{-1 p}$ \\
\hline Nightjar (Caprimulgus europaeus) & $0.917^{-1 p}$ & $0.879^{-1 p}$ \\
\hline Great Skua (Stercorarius skua) & $0.895^{-\mathrm{pk}}$ & $0.847^{-\mathrm{pk}}$ \\
\hline Garganey (Anas querquedula) & $0.879^{-\mathrm{pk}}$ & $0.823^{-\mathrm{pk}}$ \\
\hline Egyptian Goose (Alopochen aegyptiaca) & $0.856^{-\mathrm{Ip}}$ & 0.793 \\
\hline Osprey (Pandion haliaetus) & $0.825^{-\mathrm{pk}}$ & $0.744^{-\mathrm{pk}}$ \\
\hline Cetti’s Warbler (Cettia cetti) & $0.802^{-1 p}$ & $0.715^{-1 p}$ \\
\hline Firecrest (Regulus ignicapilla) & $0.788^{-\mathrm{lp}}$ & 0.719 \\
\hline Ring-necked Parakeet (Psittacula krameri) & 0.784 & $0.691^{-\mathrm{pk}}$ \\
\hline Barnacle Goose (Branta leucopsis) & $0.782^{-1 p}$ & $0.689^{-1 p}$ \\
\hline Woodlark (Lullula arborea) & $0.775^{-\mathrm{pk}}$ & $0.676^{-\mathrm{pk}}$ \\
\hline Wood Duck (Aix sponsa) & $0.769^{-1 p}$ & $0.675^{-\mathrm{pk}}$ \\
\hline Dartford Warbler (Sylvia undata) & $0.740^{-\mathrm{pk}}$ & $0.634^{-\mathrm{pk}}$ \\
\hline
\end{tabular}




\section{DisCUSSION}

The aim of this study was to explore how incorporating concepts of dispersal in SDM affects the accuracy and uncertainty of projected distributions. As it is impossible to assess accuracy of future projections, the accuracy of models calibrated with past data and projected to the current time period was assessed in an attempt to simulate many of the issues such as interactions, feedbacks, and novel climates that are problematic in modelling future species distributions. Due to these added uncertainties, expectations of accuracy were lower than they would have been for other modelling scenarios (e.g. modelling distributions in current time), and this difference was observed in the results, recording higher accuracies for the SDMs fit and projected in $1990\left(\mathrm{t}_{1}\right)$ than compared to $2010\left(\mathrm{t}_{2}\right)$. However, this result is not uncommon (Araújo et al. 2005; Veloz et al. 2012), and moreover, perfect validation of SDMs fit in future time could be conceptually impossible due to the added uncertainties and the fact that the modelled system is not closed (Araújo et al. 2005). However, as species and environmental data collection and management are improving dramatically, we are now beginning to have available data at a relevant temporal extent needed to conduct more rigorous validation of this type of study. Therefore, this research should foster subsequent studies which explicitly address both the uncertainty and accuracy associated with methods of simulating dispersal alongside species future distributions.

The selection of a statistical method was one of the most variable aspects of this research. The difference in outputs derived from the statistical methods used has long been noted as being important (Guisan and Zimmermann 2000), and several studies have identified the uncertainty in the results of different statistical methods (Reese et al. 2005; Graham et al. 2008; Elith and Graham 2009). MaxEnt better predicts the fundamental distribution of a species $\left(\mathbf{G}_{\mathbf{i}}\right)$ than the actual distribution $\left(\mathbf{G}_{\mathbf{0}}\right)$ (Figure 1.1), due to the fact 
that the algorithm uses presence-only data. GLM and RF incorporate absence data, and often constrain their predictions accordingly, meaning MaxEnt was the most likely statistical method to over-predict the distribution (as it essentially models $\mathbf{G}_{\mathbf{i}}$ ). Subsequently, under-prediction of the distribution was an issue with GLM and in particular RF, and was the primary reason the accuracy metrics did not change when the 20 dispersal models were coupled with RF and to a lesser extent GLM (Figure 2.2). This was because results were based on coupling a restrictive model of dispersal with an already under-predicted distribution. When this occurred, geographically widespread species remained relatively widespread, while the accuracy for under-predicted species couldn't be improved as the new suitable locations were either similar or exactly the same as the previous locations. This issue highlights the importance of testing several statistical models, as if only GLM or RF were used in this analysis, the results would suggest that differences in dispersal models were minimal, whereas when dispersal models were coupled with MaxEnt, results varied greatly and a number of significant differences were observed.

When the dispersal models were compared with each other (Figure 2.2, Table 2.4), in general, the more restrictive methods decreased accuracy when measured as sensitivity and TSS, as the unrealistic lack of accessibility impacted the accuracy. TSS was higher for the one-step fixed rate methods (FR1 and FR2) compared with the twostep methods (FR3 and FR4), and the probabilistic kernels with the inverse exponential PDF (PK3 and PK4) also had higher TSS and sensitivity scores compared with the other PDFs (PK1, PK2, PK5, and PK6). Surprisingly, in many instances, the limited dispersal models (L1 and L2) did not have a significantly lower accuracy than many of the other parameterized constraints (Figure 2.2, Table 2.4). These methods only penalize fragmentation in the distribution, so as long as the predicted future distribution is 
continuous, this method assumes that the species can reach these locations. With the SDMs generated at a $10 \mathrm{~km}$ resolution, much of the species distributions are connected. If these dispersal models were implemented at a finer resolution, then they would most likely have much lower sensitivity and TSS scores, as any gap in the distribution (e.g. urban area or slight change in habitat) could heavily fragment the factors that define the suitable habitat. In these instances, the choice of connectivity (rook's or Queen's) may have an important role in determining future distributions. Some differences between rook's and Queen's cases of connectivity were found in this study, particularly in the southwest of Great Britain, where islands are only connected to the mainland if Queen's case of connectivity was considered. However, with only 3 islands connected to the mainland in this manner, any change in accuracy was minimal. Subsequently, in this study, due to the spatial resolution, limited dispersal models often reported similar accuracies to unlimited dispersal, however these results are unlikely to replicate at finer resolutions and across larger extents.

The variation in area predicted present was notable. For almost every method, some difference in area was observed, and while these differences were not always significant when averaged across all 50 species, small variations in area for a specific species could have large implications on any conservation strategies for individual species. Only three methods did not vary in area predicted present by unlimited dispersal. These were three of the discrete dispersal kernels with fat-tail PDFs (DK3, DK5 and DK6). The discrete kernels are a combination of a dispersal kernel and the fixed rate method, with the maximum value drawn from a PDF used as the fixed rate of dispersal. With the fat-tailed kernels (inverse exponential and Cauchy), a value greater than the length of the country was often generated due to the relatively large number of iterations, regardless as to whether the other $99 \%$ of dispersal distances drawn from the 
PDF were within a $10 \mathrm{~km}$ radius. Subsequently, every suitable location between the maximum value was considered equal (something which is most likely not realistic of long distance dispersal events). The fact that no differences with unlimited dispersal were found for the inverse exponential and Cauchy PDFs, along with the expensive computing time and resources needed to generate these models, implies that the use of this method may be redundant (particularly for birds which are capable of travelling extensive distances). This method has been used in plant studies across much larger spatial and temporal timescales (Clark et al. 2001, Cunze et al. 2013), so future research should focus on if differences between the implementations of discrete dispersal kernels and unlimited dispersal exist for different taxa and spatiotemporal scales.

In contrast, the probabilistic kernels often resulted in less predicted area to unlimited dispersal and significantly lower TSS scores for the Gaussian and Cauchy PDFs. However, the probabilistic kernels with inverse exponential PDFs varied in area predicted present compared to unlimited dispersal, but did not differ significantly in accuracy, suggesting that this may be a more realistic method of simulating dispersal for birds compared to the discrete kernel implementation as long distance dispersal events are controlled by probabilities. In contrast to the discrete kernels, almost all of the comparisons among the probabilistic kernels resulted in a significant change in accuracy and in predicted area. The choice of PDF (and in particular the presence or absence of a fat-tail) when using a probabilistic kernel can substantially impact the accuracy of the final model. More resources should be expended for the collection of empirical data to fit a dispersal kernel, so that the choice of PDF (and any uncertainty in the user-decisions) is negated. However, if empirical data collection is not an option, effort should be directed to identifying a general framework for PDF generation (e.g., see Vittoz and Engler 2007 for a detailed discussion on the creation of dispersal kernels for European flora). 
From the comparison, no dispersal (XD) appeared to outperform the other dispersal models in terms of achieving the highest accuracy scores (Figure 2.3), and this was consistent across all the accuracy metrics except for sensitivity. This was due to XD's ability to correctly predict absences, recording the highest specificity for all 50 species, as well as the highest Kappa, TSS and PCC scores for the same reasons (Figure 2.3). However, the ability to correctly predict absences may not be as important when projecting the distribution of rare species, as a disproportionately high number of absences can exert too much influence in the metrics that consider both (Araújo et al. 2005). This issue is particularly pertinent when the test dataset used to validate the SDMs is representative of every observation across the entire study area, meaning that for sparse species, the accuracy metrics would effectively favor correctly predicting absences. This explains why XD scored so highly across many of the accuracy metrics, and why there were relatively lower Kappa and TSS scores for MaxEnt compared to GLM and RF (Table 2.3).

The rationale behind ensemble modelling in SDM is that by calculating the ensemble score, the 'signal' that one is concerned with emerges from the 'noise' associated with individual model errors and uncertainties (Araújo and New 2007). A lot of the current work with ensemble models in SDM is 'pre-thresholding', whereby suitability indices are averaged across various models and these values are used in the generation of presence/absence models (Thuiller et al. 2013; Barbet-Massin et al. 2012). For many scenarios, this type of ensemble forecast is desirable; however, many of the dispersal models used in this study require a binary presence/absence output from the statistical model in their input, and produce only a binary output. Therefore, the Ensemble Agreement Index (EAI) was developed with which to analyze these ensemble models. This index ranks full agreement for every observation (i.e. an ensemble score of 
20 out of 20 in every presence observation) as 1, and full disagreement for every observation (i.e. an ensemble score of 1 out of 20 in every presence observation) as 0 . Each species is now associated with an EAI score, and researchers can discuss dispersal potential with a greater understanding of the uncertainty of how the dispersal models align with each other.

Many of the introduced species (e.g., wood duck, ring-necked parakeet) have a relatively lower EAI score than the native species (Table 2.5). In part, this is because these species tended to have smaller original distributions, and the fact that they are not in equilibrium with the environment in 1990 due to their recent establishment. Therefore, the potential area with which they can disperse is greater than those native to the country and subsequently already occupying their niche. Raptors such as the hobby, osprey and goshawk all have platykurtic dispersion of EAI scores, suggesting that their dispersal capability varied. Similar to the introduced species, these species were most likely not in equilibrium with their environment in 1990 due to persecution (e.g. Marquiss et al. 2003), and so their predicted distribution is more fragmented, causing the slight differences in dispersal models to be exaggerated, particularly differences between the restrictive and expansive methods. For example, the areal difference between limited dispersal with rook's connectivity (L1) and Queen's connectivity (L2) for the osprey was $3,800 \mathrm{~km}^{2}$, which was $1,000 \mathrm{~km}^{2}$ greater than the average of all 50 species (Table 2.4).

There are no really low EAI scores for any of the 50 birds. In part this could be due to a relatively low uncertainty surrounding these methods, although more likely this is due to the inclusion of very similarly defined methods and possibly inflated ensemble scores (e.g., the six discrete dispersal kernel methods). When the six discrete kernel values were removed, lower scores for the species with moderate uncertainty (e.g. wood duck) were observed, whereas the species with low uncertainty (e.g. blackcap) did not 
change. Likewise, the inclusion of unlimited dispersal at this spatial extent almost negates the opportunity for an EAI score of 0 , as it is relatively unlikely that no other dispersal method will predict all the observations as absent. In practice, research will not compare as many different dispersal models with slight differences in implementation as were compared in this extensive study.

It should be noted that low uncertainty measured by the EAI does not mean that the future projections have a high accuracy. For example, Figure 2.2 shows high agreement among the 20 dispersal models when coupled with RF, but the accuracy is substantially lower than GLM or MaxEnt. However, for projections into the future (e.g. 2100) researchers will have to evaluate the uncertainty of their projections because there is no test data with which to validate. In these instances, agreement among dispersal models and other decisions in the SDM framework (e.g. statistical models) should be considered within the EAI, so that a better understanding of the output is achieved. Therefore, when generating ensemble models, different methods of implementing dispersal (or any other user-defined aspect which can be ensembled) should be selected carefully and specifically, rather than simply inputting every possible implementation.

The coarse resolution at which the land cover is generated $(1 \mathrm{~km})$ compared to the resolution at which actual bird movements occur was insufficient to generate meaningful comparisons if landscape derived metrics were used. Similarly, demographic models are very rare in SDM studies due to the paucity of comparable abundance data, and subsequently have only been applied in a few studies (e.g. Midgley et al. 2010). As such, it was not practical to include these conceptualizations of dispersal in the analysis. However, for many terrestrial animals and plants, these notions of movement may be more suitable, and more research should be conducted to see how incorporating these methods alongside SDM impacts results. 
The variation in accuracy among dispersal models suggests that the choice of dispersal model should therefore reflect the underlying purpose of the research being conducted. No dispersal (which performs well for specificity) would therefore be recommended for studies trying to identify future suitable habitat for species which have low dispersal capabilities (e.g. reptiles), or for studies aiming to find definitive locations of future abiotic suitability (without introducing the uncertainty of dispersal). While many dispersal methods scored high sensitivity (Figure 2.3), if the aim of the research is to find the potential future distribution of a species, then unlimited dispersal (UD) should be used due to its conceptual underpinnings and ability to identify every suitable location in the study area. The use of a probabilistic dispersal kernel and limited dispersal with rook's connectivity (L1) resulted in higher TSS for five of the seven non-native species. These dispersal models also recorded sensitivity scores equal to or fractionally lower $(<0.1$ difference) than unlimited dispersal. From this, it can be inferred that the statistical models for invasive species are projecting inaccessible abiotically suitable areas, and that a dispersal model that removes or controls the possibility of long distance dispersal eliminates these false positives, and improves the accuracy of metrics such as TSS. Therefore, the use of a probabilistic dispersal kernel or limited dispersal model should be used when studying invasive spread under changing climates. The use of a probabilistic dispersal kernel with an inverse exponential PDF (PK3 and PK4) resulted in slightly smaller areal extents predicted as present compared to UD and DK implementations, but no significant changes in accuracy (Table 2.4). By controlling for long distance dispersal events through probabilities, rather than the assumption that every observation between the minimum and maximum dispersal distances is equally accessible (as UD and DK implementations do), the probabilistic dispersal kernel is perhaps the most accurate 
representation to how birds disperse with their projections resulting in equally high accuracies.

\section{CONCLUSION}

The use of the 'BAM' framework within the SDM discipline to identify the factors that drive the geographic distributions of species is becoming increasingly popular. Within this framework, movement factors that refer to the processes that lead to an area being accessible (e.g., via dispersal or migration) are an important yet understudied component. The importance of incorporating dispersal alongside SDMs projecting into the future cannot be overstated, as the results of SDMs which ignore dispersal are subject to high levels of uncertainty. This is the first study that has extensively compared the accuracy and uncertainty of the methods of incorporating dispersal in SDM, and as such should serve as a foundation for studies projecting into future climates. Alongside the dispersal models, this study also compared the statistical methods, evaluated the projected 2010 distributions using multiple accuracy metrics, deconstructed species indices by species traits and reported both the accuracy and uncertainty of the models. Many of the methods of dispersal differed greatly in terms of their accuracy, particularly between the restrictive and the expansive methods, while other methods of dispersal did not differ (e.g. discrete kernels and unlimited dispersal). The use of no dispersal yielded significantly lower accuracy (TSS) when averaged across the 50 species and compared to every other method of dispersal, while conversely also recording the highest scores for the accuracy metrics which favor correct prediction of absences. Subsequently XD should only be used if the researcher has a valid reason to do so, and even then other simulations of dispersal should be incorporated alongside this. The development of the Ensemble Agreement Index allowed for the quantification of 
uncertainty among the different dispersal models, but will also provide researchers with a quantitative tool to assess the variations between their inputs for other areas of uncertainty in SDM (e.g. use of statistical method, threshold classification) or any environmental or geographic research where the main output can be binary (e.g. flood risk, urban growth). For the first time in SDM research, we have been able to compare these dispersal models based on how well they predict future geographic distributions. Not only has this research provided practitioners with a product with which to select a dispersal model to use, saving their time, resources and research efforts, it has also resulted in a better understanding of the effects of both the accuracy and uncertainty on projected distributions, extinction rates and dispersal patterns. 


\section{CHAPTER 3: QUATERNARY RANGE SHIFTS: A QUANTITATIVE ASSESSMENT OF THE METHODS USED TO MODEL PLANT DISPERSAL}

\section{INTRODUCTION}

Due to the ongoing and unprecedented global effects of climate change across a multitude of landscapes (such as novel combinations of climate scenarios and the extinction of keystone species), understanding where species could be found under future climate scenarios is one of the most pressing aspects of biodiversity conservation (Lomolino and Heany 2004). By identifying the potential future geographic ranges of species, measures can be taken to ensure that suitable habitat persists, and that it remains accessible to any future dispersing populations. Furthermore, by understanding which factors define a species range, predictions can be made about the species resilience, as well as more accurate estimations of range constriction, expansion, or extinction. Ultimately, this knowledge will allow the necessary resources to be directed effectively and efficiently towards protecting and preserving those species that require them.

Species distribution models (SDMs) are an important tool for studying the geographic distribution of a wide range of taxa, providing a robust framework to quantitatively assess the relationship between species distributions and the environment (Franklin 2009). Moreover, these statistical relationships can be extrapolated in time and space, and as a result, SDMs are increasingly being used to project species range shifts under changing climates (Pearson and Dawson 2003; Hijmans and Graham 2006; Guisan and Thuiller 2005; Araújo and Luoto 2007; Wiens et al. 2011). This has allowed researchers to predict how species might respond to climate change, with many projections identifying dramatic range constrictions and a large number of global extinctions (Thomas et al. 2004; Urban 2015). While SDMs projected into the future can 
provide unparalleled insights into how the geographic ranges of species will react to climate change, the extrapolation of these species-environment relationships introduces uncertainties related to novel environmental combinations, species interactions, future climate change scenarios, and in particular the dispersal capacity of the species.

When projecting into the future, it is important that the SDM framework not only addresses the abiotic and biotic suitability of the new environment, but also whether the environment is accessible. The 'BAM' framework, developed by Soberón and Peterson (2005), illustrates this concept, with the actual distribution of a species found only where suitable biotic (B), abiotic (A), and movement ( $\mathbf{M}$ - in terms of accessibility) factors coincide. For future projections, this is achieved by coupling a statistical model (which projects abiotic and biotic suitability) with a dispersal model (which projects accessibility). Various methodologies have been developed to derive dispersal rates of the population over time (Table 1.4), and these models are then subsequently used to constrain the projected abiotic and biotic suitability (Franklin 2010; Miller and Holloway 2015). With such variation in the methodologies, the choice of dispersal model can greatly impact the results and generate uncertainty in future projections. For example, in a study projecting the 2080 distribution of 140 European flora species, Cunze et al. (2013) found significant differences between a dispersal model that assumed unlimited movement and one that used a discrete dispersal kernel. Using two virtual plant species, Engler and Guisan (2009) found a 95\% areal difference in the projected distributions from a model which ignored dispersal compared to one which used a probabilistic dispersal kernel. Recently, Holloway et al. (2016) compared 20 implementations of dispersal for 50 British breeding birds from 1990 to 2010. They found that the choice of dispersal model and how it is implemented could significantly influence the results both in terms of accuracy and the area predicted present. The choice of dispersal model 
undoubtedly introduces uncertainty into projections, and while a handful of studies have compared the results (Engler and Guisan, 2009; Cunze et al. 2013; Holloway et al. 2016) more extensive testing across different taxa and spatial scales is needed.

When extrapolating species-environment relationships across time, there is the assumption that the biotic and abiotic factors will remain constant for every intermediate time period. While a postulate of equilibrium is necessary for projecting the model in space and time (Guisan and Thuiller 2005), for long time periods (e.g. 100 years) it is highly unlikely that the abiotic factors will remain the same. Simulating dispersal as a multiple-step process (e.g., from $t_{1}$ to $t_{1 a}$ to $t_{1 b}$ to $t_{2}$ ) rather than the traditional one-step process (e.g., from $t_{1}$ to $t_{2}$ ), accounts for some of the inherent uncertainty related to the assumption of homogeneity of abiotic and biotic factors. For models simulating dispersal as a multiple-step process, at each intermediate time-step the dispersal model is coupled with a corresponding statistical model of abiotic and biotic suitability. The next dispersal event then originates from the area predicted present based on suitability and accessibility for this time period (Figure 3.1). If the abiotic and biotic conditions alter significantly, then the number of steps used to model dispersal could greatly influence the projected distributions. For example, in a study investigating the effects of climate change on 336 Proteceae species in South Africa, Midgely et al. (2006) simulated dispersal using a fixed rate method of $4 \mathrm{~km} /$ year or $1.5 \mathrm{~km} /$ year for species dispersed by wind or rodents respectively. They found that the resulting ranges of species in 2050 were larger by approximately $10 \%$ when dispersal was simulated as a single 50 year time-step compared to when dispersal was simulated in decadal time-steps due to the reduced abiotic suitability at intermediate temporal periods. Similarly, Holloway et al. (2016) also found significant differences in area and accuracy among implementations of a fixed rate method of dispersal when simulated as a one-step process compared to a two-step process 
for 50 British bird species. With studies highlighting large differences in the future geographic ranges of species, the uncertainty arising from modelling future distributions in multiple time-steps needs to be explored further.
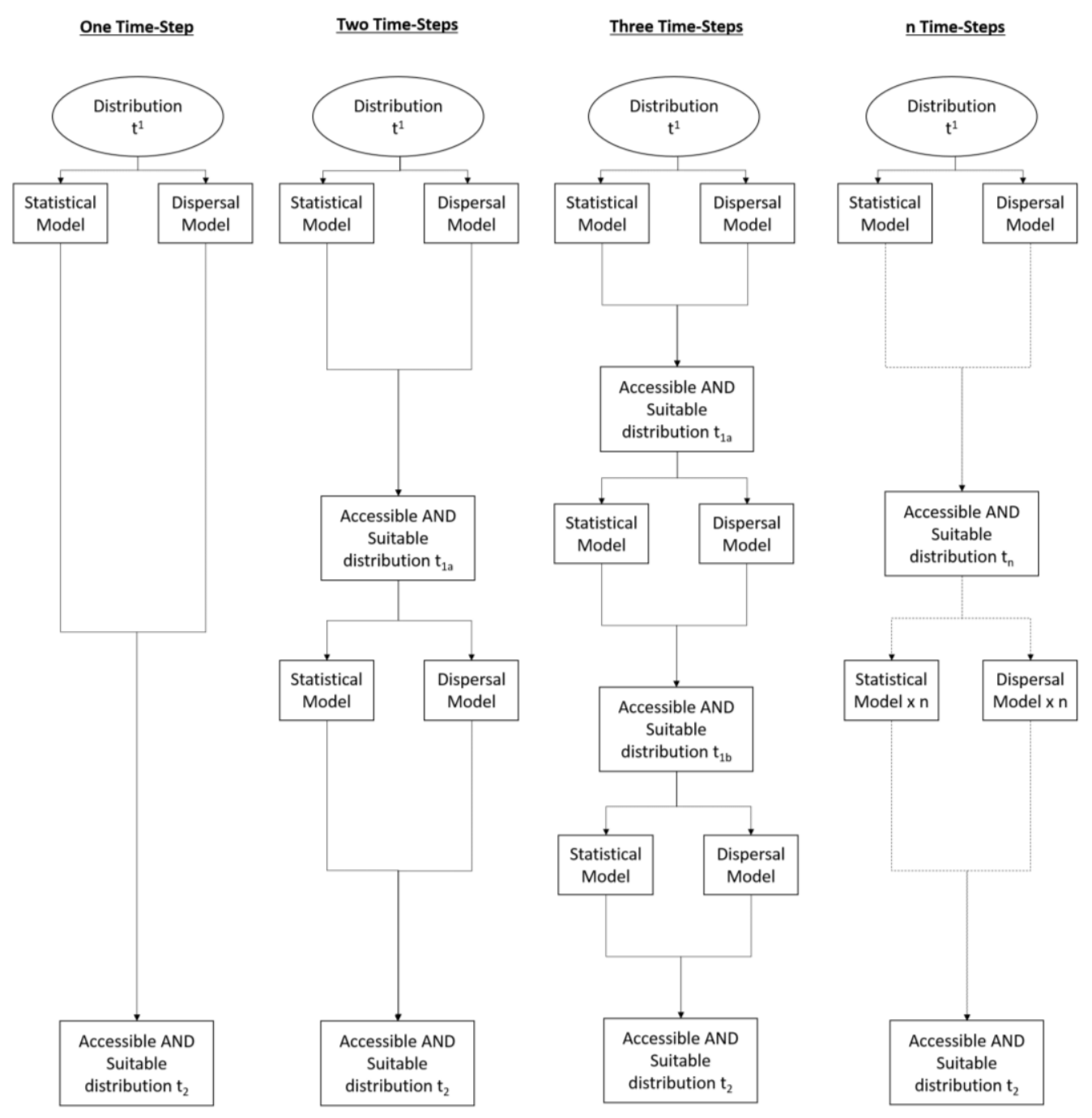

Figure 3.1: Flow chart illustrating the method behind using multiple dispersal steps in the SDM framework. 
Recently, researchers have begun using past data to project species-environment relationships into the present, with the current distribution of species used as independent test data with which to assess the accuracy of projections (e.g. Araújo et al. 2005). For example, Veloz et al. (2012) used fossilized pollen from 21 to $15 \mathrm{ka}$ BP to project the $20^{\text {th }}$ Century geographic ranges of taxa in North America, and evaluated the accuracy of the statistical methods used to fit the species-environment relationships. While they found issues in terms of the predictive capability of SDMs with species which currently inhabit climates with no analog when compared to the last glacial maximum (21ka BP), SDMs for species whose niches remained stable performed well. Paleo-vegetation data can be used in SDM to systematically explore the uncertainty and accuracy of the different dispersal models across a suitably long time period, and in particular to assess the use of simulating dispersal as a multiple-step process.

\section{MeTHODOLOGY}

\section{Data Collection}

Site-level pollen data for 29 genera from $12 \mathrm{ka}$ BP were obtained from the Neotoma paleoecology database (www.neotomadb.org) via Williams et al. (2004). Pollen percentages were converted to a binary product representing the presence or absence of the genera at the specific site locations. This resulted in 281 presence/absence observations with which to fit the genera-environment relationships. The pollen data only included taxa recognizable to the genus level (with the exception of palynologically indistinguishable genera such as Ostrya/Carpinus - Williams et al. 2004). Herein, any reference to 'species' in a methodological context (e.g. 'species' distribution model) should be considered synonymous with 'genera', as the statistical and dispersal models will be fit the same as if working with species-level data. If the fitting of genera- 
environment relationships does result in a unique approach or assumption, this will be explicitly stated. The current geographic ranges of species were obtained from Critchfield and Little (1966) and Little (1971, 1976, 1977, 1978), and merged to represent the genuslevel geographic ranges for the late $20^{\text {th }}$ Century.

Downscaled CCSM3 Paleoclimate Simulations for North America from 12ka BP to the current period were obtained from Veloz et al. (2012) and Blois et al. (2013). These climate variables have a spatial resolution of $50 \mathrm{~km}$, and were obtained at a 1000 year temporal resolution. The variables in this dataset included mean daily maximum and minimum temperature and mean precipitation for spring (March to May), summer (June to August), autumn (September to November), and winter (December to February), as well as the bioclimatic variables 4, 7, and 15 (Hijmans et al. 2005). For a detailed report on how these variables were generated, see Veloz et al. (2012) or Blois et al. (2013).

\section{Species Distribution Modelling}

Abiotic suitability was estimated using three statistical methods and accessibility (movement suitability) was determined from seven dispersal models. A binary layer of suitable or unsuitable abiotic habitat was created from the statistical models, and a binary layer of accessible or inaccessible was generated from the dispersal models. These outputs were then combined to create a binary layer of presence or absence which identified habitat that was both abiotically suitable and accessible. Biotic factors were excluded from analysis, as interactions can vary between species of the same genus and often occur at much finer spatial scales than abiotic and movement factors. However, for methods of incorporating biotic interactions in SDM see Wisz et al. (2013). 


\section{Statistical Modelling}

To predict abiotic suitability, three commonly applied statistical methods known for generating reliable results were considered: Generalized Linear Model (GLM), Random Forest (RF), and Maximum Entropy (MaxEnt). For each genera, the binary pollen data were fit to the climate data from 12ka BP. Stepwise selection of the environmental variables based on minimizing Akaike Information Criterion (AIC) was used to select one GLM, classification random forest was used to generate one RF model, and one MaxEnt model using default settings (with the exception of 5000 minimum iterations) was created (see Phillips and Dudik 2008). A threshold was used to convert the suitability index into a binary measure of presence or absence. The threshold was selected based on prevalence of presences in the 12ka BP data for GLM and RF, and the maximum training sensitivity plus specificity threshold was used for MaxEnt. Thresholds were selected based on data type (e.g. presence/absence or presence-only) and guidelines recommended by similar studies (Liu et al. 2005, Hu and Jiang 2011).

The genera-environment relationships were then extrapolated to 0ka BP. Each of the three statistical methods deals with extrapolation (predicting in future, and sometimes novel or non-analogue, climate conditions) differently; GLM extrapolates by continuing the fitted trend beyond the last observation, MaxEnt acts consistently and is 'clamped' so it extrapolates in a horizontal line from the fit at the most extreme environmental value in the training data, and random forest extrapolates at a constant value from the last 'known' site (Elith and Graham 2009). The output from these models can be considered as implicitly representing unlimited dispersal, and the dispersal models considered here essentially constrain these model predictions according to different conceptualizations of dispersal. The same methodology, genera-relationships and thresholds were used for all future projections (0ka BP and any intermediate time-periods). 


\section{Dispersal Modelling}

Within SDM, there are two predominant conceptualizations of how to simulate dispersal at the population level: 'all or nothing' and 'parameterized constraints' (Franklin 2010; Miller and Holloway 2015). All or nothing applies a simple assumption of either unlimited dispersal or no dispersal, while parameterized constraints apply a rate of dispersal from the current spatial extent of the distribution. All methods, with the exception of the probabilistic kernel (simulated using kernel function in MigClim Engler et al. 2013), were simulated using $\mathrm{R}$ functions generated by Holloway et al. (2016) (Appendix 2).

\section{All or Nothing}

Unlimited dispersal (UD) assumes that all suitable habitat in the future geographic space is accessible, while no dispersal (XD) assumes that only currently suitable habitat is accessible in future projections. Limited dispersal assumes that only contiguous areas of suitable habitat in future geographic space that overlap with areas of suitable habitat in current geographic space are accessible, and this can be varied by the assumption of connectivity, with continuous habitat defined by either four-neighbors (L1) or eightneighbors (L2).

\section{Parameterized Constraints}

Three implementations of parameterized constraints were modelled: fixed rate (FR), discrete dispersal kernel (DK), and probabilistic dispersal kernel (PK). All of these methods require a rate of dispersal in order to be properly fit. Empirically derived dispersal rates would ideally be used due to the variability and uncertainty of fitting theoretically derived dispersal kernels (Holloway et al. 2016), however, compiling dispersal information for every species in a region would be extremely costly and 
inefficient. In addressing this issue, Vittoz and Engler (2007) performed an extensive literature review to identify recorded dispersal distances for European flora. They then attributed to each species a dispersal type, which was a function of the dispersal mode (e.g. wind, animal) and species traits (e.g. height). Empirical distances could then be drawn upon from this database for species which have no observed data but similar dispersal mechanisms. Following Vittoz and Engler's (2007) methodology, a database of seed dispersal distances (including mean and maximum values) were compiled for North American flora with the findings used to inform the dispersal rates for genera used in this research.

Species traits were classified based on the U.S. Forest Service's delineation of life-form category. Due to the high overlap within the tree and shrub categories between species of the same genus; all 29 genera used in the final analysis were classified as Tree/Shrub. If multiple dispersal agents acted for a species, the dispersal agent for which the study was investigating was chosen, or if an agent was not identified, the predominant method of dispersal for that species was chosen. Dispersal types were based on Vittoz and Engler's (2007) classification, as dispersal vectors do not differ substantially between North America and Europe. For a dispersal rate to be representative of a population over time, it should be a function of the dispersal distance equal to the net reproductive rate $\left(\mathrm{R}_{0}\right)$, multiplied by the temporal period $(\mathrm{T})$ divided by the age at which the species first breeds (A). Therefore, information on the age at which a tree could first produce seeds was also compiled, and the mean (categorized by dispersal type) of these values was used to calculate the generation time. $\mathrm{T}$ is therefore representative of the time-period (e.g. 1000 years) divided by the age of seed production for a tree. While there are many other factors that can control whether a seed becomes established or not (e.g. competition, space), it had to be assumed that seeds were allowed to establish immediately, which 
most likely resulted in a slightly faster rate of dispersal than would be observed in nature (n.b. that this is a common assumption in SDM and dispersal studies).

Fixed rate (FR) involves using a rate of dispersal and applying this as a timebased distance buffer around the current distribution. Following Vittoz and Engler (2007), the mean dispersal distance was taken as the $80^{\text {th }}$ percentile of all the mean dispersal distances recorded in the literature review. By using the $80^{\text {th }}$ percentile of the dispersal distances, the values used in this research are not affected by any extreme dispersal events which could inflate the mean. A square buffer was then applied around the current distribution which matched the rate of dispersal.

The dispersal kernels were fit using the empirical data collected from the literature review. The discrete dispersal kernel (DK) is estimated according to the maximum value drawn from a random sample of the size of the net reproductive rate $\mathrm{R}_{0}$ (Clark et al. 2001, Cunze et al. 2013). $\mathrm{R}_{0}$ was set to 10 million, calculated by recording the average density of trees and the average number of seeds produced by a single tree and extrapolating this to a resolution of $50 \mathrm{~km}$. While these values were substantially higher than 10 million (e.g. 100 trillion), there was no statistical difference between the maximum value drawn from 10 million or 100 trillion (data not shown), although there was a significant difference in the processing time. Therefore it was deemed sufficient to use the maximum dispersal distance from 10 million seeds. The probabilistic dispersal kernel (PK) assigns each grid a conditional probability to be colonized based on the observed distances, and from this probability generates a binary layer of presence or absence. 


\section{Time-Steps}

For each dispersal model, six different temporal intervals were simulated (Table 3.1). At each time step, a statistical model projecting the abiotic suitability and a dispersal model projecting the accessibility were coupled (Figure 3.1). Dispersal was then simulated onwards from presence locations projected for that intermediate time. The impact of multiple time-steps was investigated on all the methods except UD as there would be no difference among implementations of multiple steps.

Table 3.1: The temporal resolution of each time-step implementation

\begin{tabular}{ccc}
\hline Time-Steps & Temporal Resolution & Intermediary Time-Periods \\
\hline $\mathbf{1}$ & 12,000 years & NA \\
\hline $\mathbf{2}$ & 6,000 years & $6 \mathrm{ka} \mathrm{BP}$ \\
\hline $\mathbf{4}$ & 4,000 years & $8 \mathrm{ka}, 4 \mathrm{ka} \mathrm{BP}$ \\
\hline $\mathbf{6}$ & 3,000 years & $9 \mathrm{ka}, 6 \mathrm{ka}, 3 \mathrm{ka} \mathrm{BP}$ \\
\hline $\mathbf{1 2}$ & 2,000 years & $10 \mathrm{ka}, 8 \mathrm{ka}, 6 \mathrm{ka}, 4 \mathrm{ka}, 2 \mathrm{ka} \mathrm{BP}$ \\
\hline & 1,000 years & $\begin{array}{c}11 \mathrm{ka}, 10 \mathrm{ka}, 9 \mathrm{ka}, 8 \mathrm{ka}, 7 \mathrm{ka}, 6 \mathrm{ka}, \\
5 \mathrm{ka}, 4 \mathrm{ka}, 3 \mathrm{ka}, 2 \mathrm{ka}, 1 \mathrm{ka} \mathrm{BP}\end{array}$ \\
\hline
\end{tabular}

\section{Model Accuracy}

Two test datasets were generated to evaluate the models. The first dataset consisted of 1000 randomly generated points within the study area, and the second dataset consisted of a data point for each $50 \mathrm{~km}$ grid in the study area. Presence or absence of a genus was identified using the observed current distribution (Critchfield and Little 1966; Little 1971, 1976, 1977, 1978). Five threshold-dependent accuracy metrics were then used to evaluate the models (Table 2.2). Sensitivity and specificity measure the 
proportion of correctly predicted presences and absences respectively. Proportion correctly classified (PCC) measures the total number of correctly predicted presences and absences, while the true skill statistic (TSS) measures the combined sum of sensitivity and specificity. Kappa is a measure of categorical agreement that describes the differences between the observed agreement and chance agreement. The Area Under the Curve (AUC) was the only threshold independent metric used. AUC is calculated by summing the area under the receiver operating curve (ROC) plot, which is a graph of the false-positive error rate on the $\mathrm{x}$-axis plotted against the true positive rate on the $\mathrm{y}$-axis. For a full discussion on accuracy metrics, see Franklin (2009).

\section{RESULTS}

Table 3.2 shows the mean and standard deviation of the accuracy scores for the three statistical methods fit on the 12ka BP genera and environmental data. The generaenvironment relationships were assessed for their accuracy when projected in current time (12ka BP) and future time (0ka BP) with unlimited dispersal. Interestingly, MaxEnt scored the highest sensitivity (proportion of correctly predicted presences) when projected in current time (12ka BP) for the 29 genera, but this value halved when the relationships were projected into the future (0ka BP). For GLM and RF, accuracy for the 29 genera improved when projected into the future, and both models outperformed MaxEnt in all the accuracy metrics with the exception of specificity (proportion of correctly predicted absences). This suggests that MaxEnt is seriously under-predicting the future geographic distributions of the taxa. GLM has the highest sensitivity for future scenarios. Sensitivity cannot be improved when the statistical model is coupled with a dispersal model due to the fact that dispersal constrains the projections based on accessibility. Therefore, the remaining statistical analysis will be presented with only 
GLM due its ability to achieve high sensitivity scores, allowing for the possibility of validation among the dispersal models. Finally, small differences in the accuracy of future projections were observed when all future observations (UD_ALL) or 1000 randomly generated points (UD_1000) were used as the test data. As such, accuracy will be assessed using all observations in order to give the most robust measure of evaluation.

Table 3.2: The mean and standard deviation of the accuracy values recorded for models projected from $12 \mathrm{ka}$ BP to $0 \mathrm{ka}$ BP with an assumption of unlimited dispersal. Test data for the future projections consisted of every observation in North America (UD_ALL) and 1000 randomly generated points (UD_1000). Data were partitioned into 80:20 training:test data for the 12ka BP model (see Fielding and Bell 1997), although final genera-environment relationships were fit on all observations. Accuracy metrics of area under the curve (AUC), sensitivity, specificity, proportion correctly classified (PCC), true skill statistic (TSS) and Kappa were recorded.

\begin{tabular}{|c|c|c|c|c|c|c|}
\hline & $\mathbf{A U C}$ & Sensitivity & Specificity & PCC & TSS & Kappa \\
\hline \multicolumn{7}{|l|}{ 12ka BP } \\
\hline \multirow[t]{2}{*}{ MaxEnt } & 0.656 & 0.782 & 0.495 & 0.630 & 0.276 & 0.174 \\
\hline & $(0.116)$ & (0.099) & $(0.272)$ & $(0.180)$ & $(0.275)$ & $(0.154)$ \\
\hline \multirow[t]{2}{*}{ GLM } & 0.633 & 0.629 & 0.635 & 0.668 & 0.264 & 0.220 \\
\hline & $(0.124)$ & $(0.336)$ & $(0.305)$ & $(0.235)$ & $(0.250)$ & $(0.232)$ \\
\hline \multirow[t]{2}{*}{$\mathrm{RF}$} & 0.668 & 0.588 & 0.739 & 0.704 & 0.328 & 0.263 \\
\hline & $(0.152)$ & $(0.331)$ & $(0.238)$ & $(0.196)$ & $(0.314)$ & $(0.280)$ \\
\hline \multicolumn{7}{|c|}{$0 \mathbf{k a}^{\text {UD_ALL }}$} \\
\hline \multirow[t]{2}{*}{ MaxEnt } & 0.650 & 0.348 & 0.922 & 0.725 & 0.271 & 0.239 \\
\hline & $(0.135)$ & $(0.303)$ & $(0.053)$ & $(0.175)$ & (0.301) & $(0.235)$ \\
\hline \multirow[t]{2}{*}{ GLM } & 0.699 & 0.679 & 0.691 & 0.711 & 0.370 & 0.320 \\
\hline & $(0.117)$ & $(0.260)$ & $(0.272)$ & $(0.166)$ & (0.278) & $(0.234)$ \\
\hline \multirow[t]{2}{*}{$\mathrm{RF}$} & 0.733 & 0.649 & 0.765 & 0.749 & 0.414 & 0.374 \\
\hline & $(0.125)$ & $(0.272)$ & $(0.249)$ & $(0.176)$ & $(0.330)$ & $(0.267)$ \\
\hline \multicolumn{7}{|c|}{$0 \mathbf{k a}^{\mathrm{UD} \_1000}$} \\
\hline \multirow[t]{2}{*}{ MaxEnt } & 0.645 & 0.336 & 0.921 & 0.727 & 0.258 & 0.225 \\
\hline & $(0.134)$ & $(0.301)$ & $(0.053)$ & $(0.170)$ & (0.301) & $(0.233)$ \\
\hline \multirow[t]{2}{*}{ GLM } & 0.700 & 0.680 & 0.691 & 0.710 & 0.371 & 0.318 \\
\hline & $(0.117)$ & $(0.259)$ & $(0.273)$ & $(0.168)$ & (0.277) & $(0.236)$ \\
\hline \multirow[t]{2}{*}{$\mathrm{RF}$} & 0.736 & 0.653 & 0.760 & 0.748 & 0.413 & 0.369 \\
\hline & $(0.123)$ & $(0.273)$ & $(0.253)$ & (0.179) & (0.339) & $(0.272)$ \\
\hline
\end{tabular}


Dispersal information on 105 flora species currently found in North America was recorded (Appendix 4). Quantitative investigations were rare, and subsequently dispersal distances based on observations and anecdotal knowledge had to be included. Where ranges were documented (e.g. $50 \%$ of seeds dispersed between $10 \mathrm{~m}$ and $20 \mathrm{~m}$ ), the upper value was recorded for dispersal distances while the lower value was recorded for age of seed production. The $80^{\text {th }}$ percentile of the mean dispersal distances varied from $1.1 \mathrm{~m}$ for species with ballistic dispersal to $2,454 \mathrm{~m}$ for species that are ingested by animals and then later excreted (Figure 3.2, Table 3.3). The dispersal kernels (Figure 3.2) show the variability in overall dispersal distances, with the two animal dispersal modes (dyszoochory and endozoochory) generally recording distances substantially higher than the other dispersal mechanisms, although the longest dispersal distance recorded was 30,000m for the Populous alba (van der Pij1 1972 cited in Gucker 2010b) which has a dispersal mode classified as trichometeorochory.

Table 3.3: Values represent $80^{\text {th }}$ percentile of mean and maximum dispersal distances, and mean age of maturity.

\begin{tabular}{|c|c|c|c|c|}
\hline \multicolumn{2}{|c|}{$\begin{array}{c}\text { Dispersal } \\
\text { Distances [m] }\end{array}$} & \multirow[t]{2}{*}{ Dispersal Mode } & \multirow[t]{2}{*}{ Description of Dispersal Mode } & \multirow[t]{2}{*}{$\begin{array}{l}\text { Age of } \\
\text { Maturity }\end{array}$} \\
\hline Mean & Max & & & \\
\hline 3.8 & 80 & Autochory & No vector, seeds fall to the ground. & 4 \\
\hline 1.1 & 9.3 & Ballochory & $\begin{array}{l}\text { The explosion of the fruit ejects the } \\
\text { seeds. }\end{array}$ & 3 \\
\hline 190 & 12,500 & Dyszoochory & $\begin{array}{c}\text { Seeds foraged and stocked by } \\
\text { animals. }\end{array}$ & 9 \\
\hline 2454 & 10,006 & Endozoochory & $\begin{array}{l}\text { Seeds digested and excreted by } \\
\text { animals. }\end{array}$ & 4 \\
\hline 117 & 520 & Pterometeorochory & Winged seeds dispersed by wind. & 18 \\
\hline 940 & 24,028 & Trichometeorochory & $\begin{array}{l}\text { Hairy structured seeds dispersed by } \\
\text { wind. }\end{array}$ & 1 \\
\hline
\end{tabular}



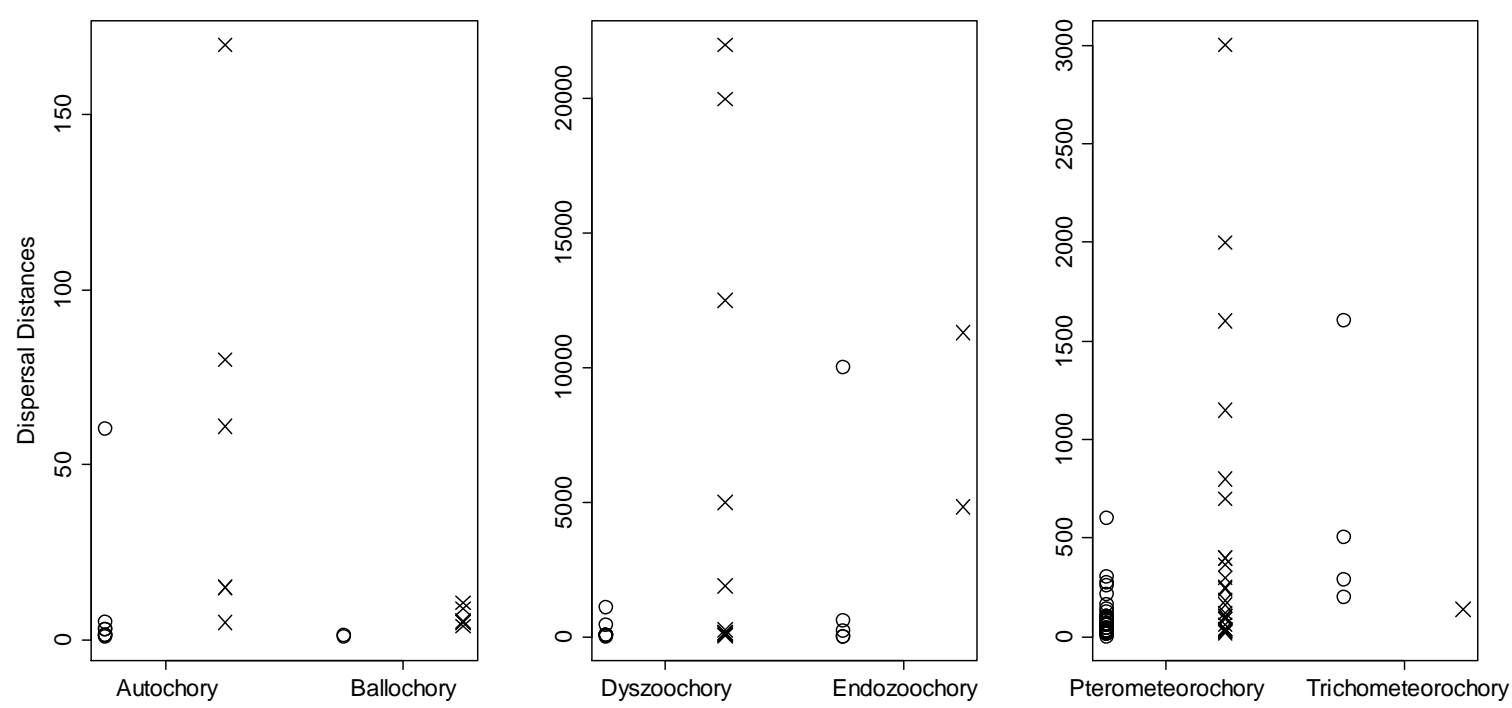

Figure 3.2: Dispersal kernels for the six dispersal types recorded for the genera in the study. o represents mean dispersal distances, $x$ represents maximum dispersal distances. Outlier of $30,000 \mathrm{~m}$ is missing from the trichometeorochory column.

Figure 3.3 shows the range of True Skill Statistic (TSS; Sensitivity + Specificity 1) scores for the 29 genera for each dispersal model (Figure 3.3 - Top Panel) and each implementation of time-steps (Figure 3.3 - Bottom Panel). The differences among the dispersal models become more pronounced once the number of time-steps is increased (Figure 3.3 - Top Panel). The fixed rate (FR) method has the largest range of TSS scores, recording the highest median TSS when dispersal is modelled in one time-step, but the second lowest median (after no dispersal) when it is modelled in twelve time-steps. Interesting, no dispersal (XD) has a higher upper-quartile score for the 29 genera than all the other methods, although the median value is often substantially lower.

There is less variation in the interquartile and overall ranges when TSS scores are compared across time-steps (Figure 3.3 - Bottom Panel). Limited dispersal with an eightneighbor rule (L2) and the discrete dispersal kernel (DK) show no difference in TSS values when compared across time-steps. The median TSS values for L1 show a lower 
score for steps which model abiotic suitability at 8ka or 4ka BP (3TS, 6TS, and 12TS), suggesting a possible shift in suitable abiotic conditions at one of these time-periods.

Similar results are observed when the area predicted present $\left(\mathrm{km}^{2}\right)$ is compared (Table 3.4). The average area for the 29 genera considered together identifies that all models (with the exception of the discrete dispersal kernels with multiple time-steps) differed significantly in area predicted present by unlimited dispersal. Both implementations of limited dispersal (L1 and L2) and the probabilistic kernel (PK) when modelled with multiple time-steps (with the exception of the twelve step PK) did not differ significantly in area compared to when dispersal was modelled as a one-step process.

Similar results are observed when the area predicted present $\left(\mathrm{km}^{2}\right)$ is compared among dispersal models and time-step implementations (Table 3.4, Figure 3.5). The average area for the 29 genera considered together identifies that all models (with the exception of the discrete dispersal kernels with multiple time-steps) differed significantly in area predicted present by unlimited dispersal. Both implementations of limited dispersal (L1 and L2) and the probabilistic kernel (PK) when modelled with multiple time-steps (with the exception of the twelve step PK) did not differ significantly in area compared to when dispersal was modelled as a one-step process. 


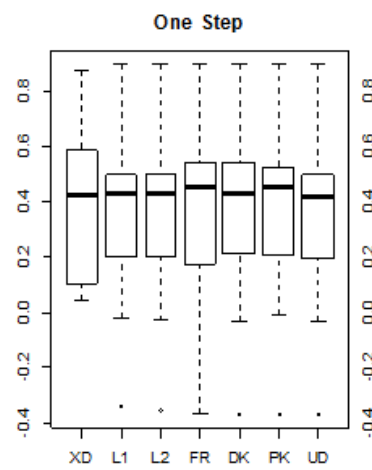

XD

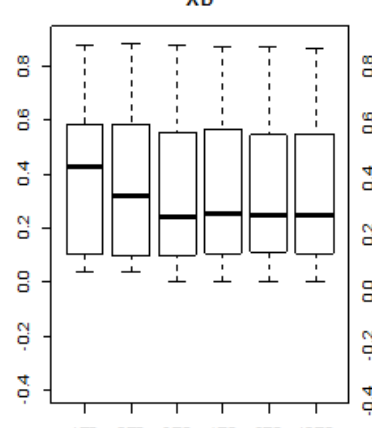

1TS 2TS 3TS 4TS 6 TS $12 T S$
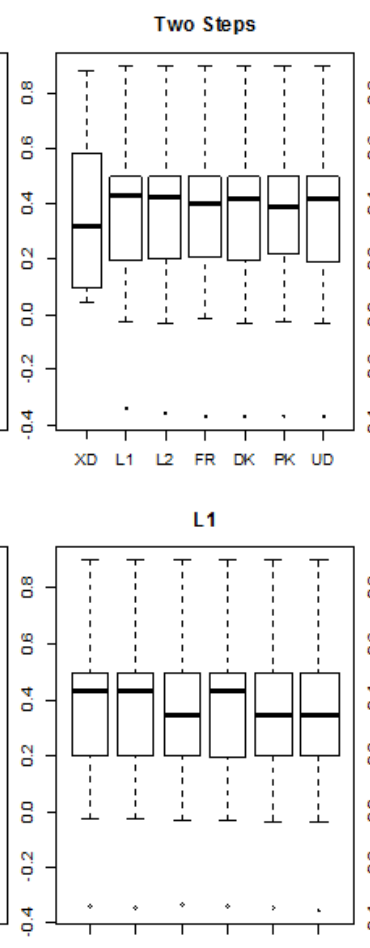

1TS 2TS 3TS 4TS BTS $12 T$

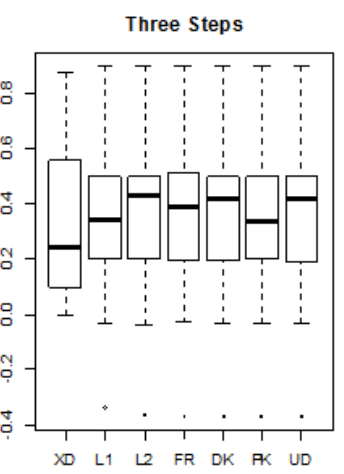

L2

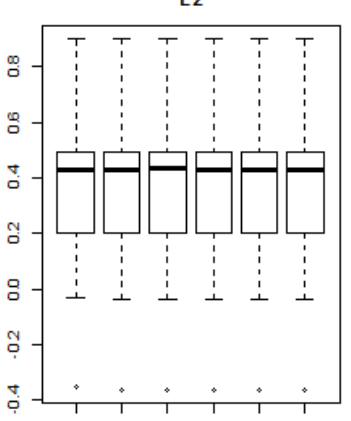

ITS 2 TS 3 TS 4 TS 6 OTS $12 T S$

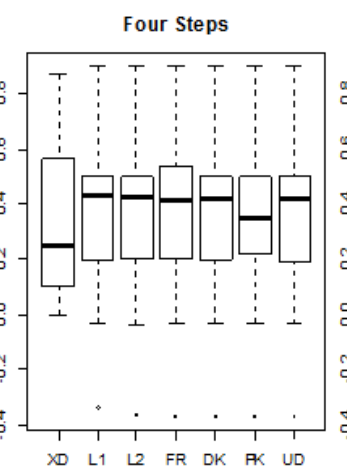

FR

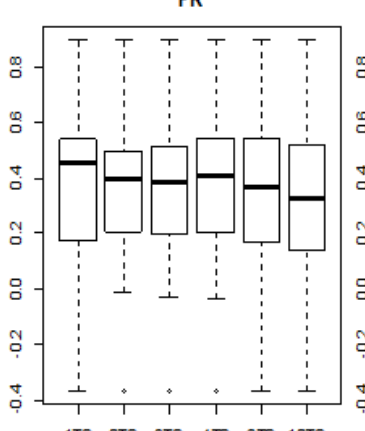

1TS 2TS 3TS 4 TS 6 TS $12 T S$

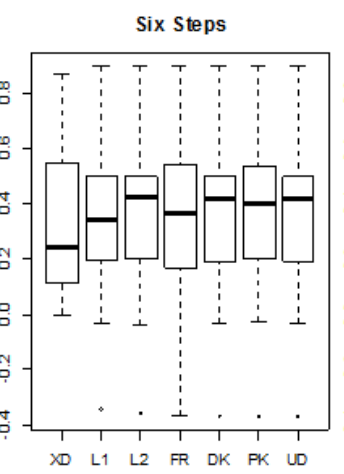

DK

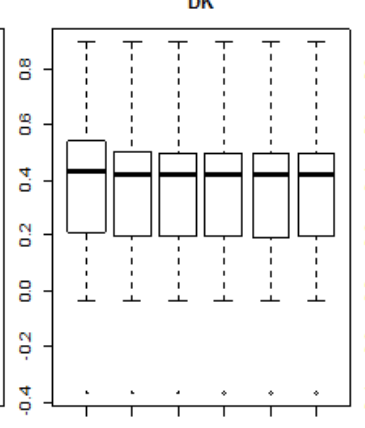

TTS 2TS 3TS 4TS

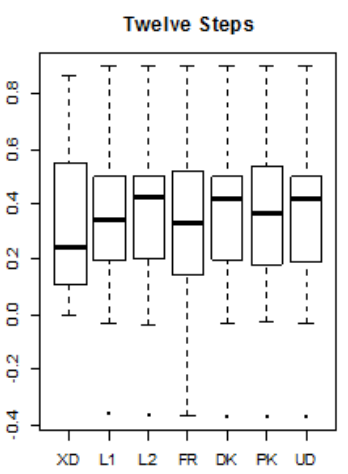

PK

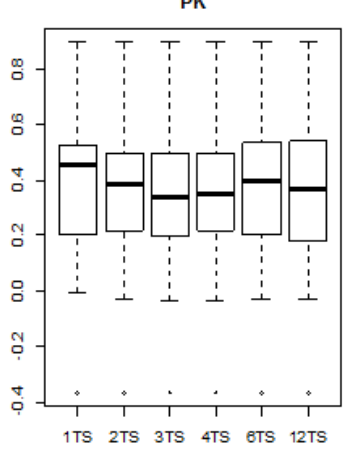

Figure 3.3: Boxplots showing the range of True Skill Statistic scores (TSS) for dispersal models with the same time-step (TS) implementation (Top Panel) and for dispersal models with different time-step implementations (Bottom Panel). Dispersal models include no dispersal (XD), limited dispersal with four-neighbor connectivity (L1), limited dispersal with eight-neighbor connectivity (L2), fixed rate (FR), discrete dispersal kernel (DK), probabilistic dispersal kernel (PK), and unlimited dispersal (UD). 


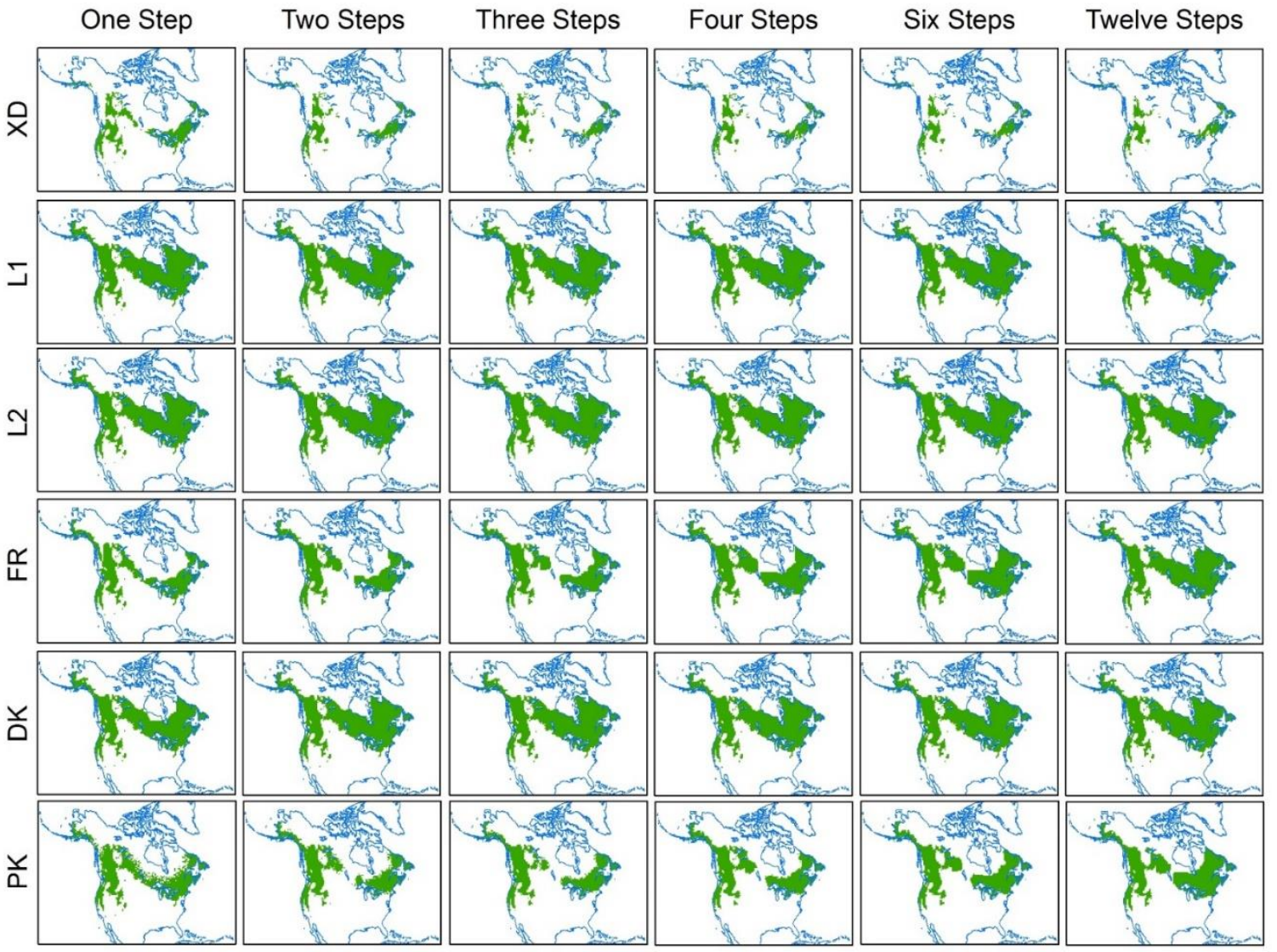

Figure 3.4: The projected distribution of Abies for each of the six dispersal models (rows) and each of the six time-step implementations (columns). Dispersal models include no dispersal (XD), limited dispersal with four-neighbor connectivity (L1), limited dispersal with eight-neighbor connectivity (L2), fixed rate (FR), discrete dispersal kernel (DK), and probabilistic dispersal kernel (PK). 
Table 3.4: The area $\left(\mathrm{km}^{2}\right)$ predicted present by each dispersal model implemented at different time steps (TS). Dispersal models include no dispersal (XD), limited dispersal with four-neighbor connectivity (L1), limited dispersal with eight-neighbor connectivity (L2), fixed rate (FR), discrete dispersal kernel (DK), probabilistic dispersal kernel (PK), and unlimited dispersal (UD). * Significantly different in area to UD $\alpha=0.01 . \Delta$ Significantly different to TS $1 \alpha=0.01$. Significance was calculated using matched paired t-tests.

\begin{tabular}{|c|c|c|c|c|c|c|}
\hline & TS1 & TS2 & TS3 & TS4 & TS6 & TS12 \\
\hline XD & $4224224 * \Delta$ & $3947328 *^{\Delta}$ & $3818621^{*}$ & $3761466^{* \Delta}$ & $3672586^{*}$ & $3548190 * \Delta$ \\
\hline L1 & $7473621 * \Delta$ & $7490603 * \Delta$ & $7464052^{* \Delta}$ & $7469483 * \Delta$ & $7466638^{*}{ }^{\Delta}$ & $7482069 * \Delta$ \\
\hline L2 & $7514310^{*} \Delta$ & $7532759 * \Delta$ & $7521121^{* \Delta}$ & $7516466^{* \Delta}$ & $7519569 *^{\Delta}$ & $7511293 *^{\Delta}$ \\
\hline FR & $6081897 * \Delta$ & $6412069 *^{\Delta}$ & $6655086^{* \Delta}$ & $6905603^{*}$ & $7121638^{*}$ & $7401897 *^{\Delta}$ \\
\hline DK & $7309569 * \Delta$ & $7606207^{\Delta}$ & $7624224^{\Delta}$ & $7628793^{\Delta}$ & $7608707^{\Delta}$ & $7629483^{\Delta}$ \\
\hline PK & $6373362 * \Delta$ & $6400345^{*}{ }^{\Delta}$ & $6349655^{* \Delta}$ & $6442672 * \Delta$ & $6612414^{*}{ }^{\Delta}$ & $6921897 *^{\Delta}$ \\
\hline
\end{tabular}

UD $7633621^{\Delta}$

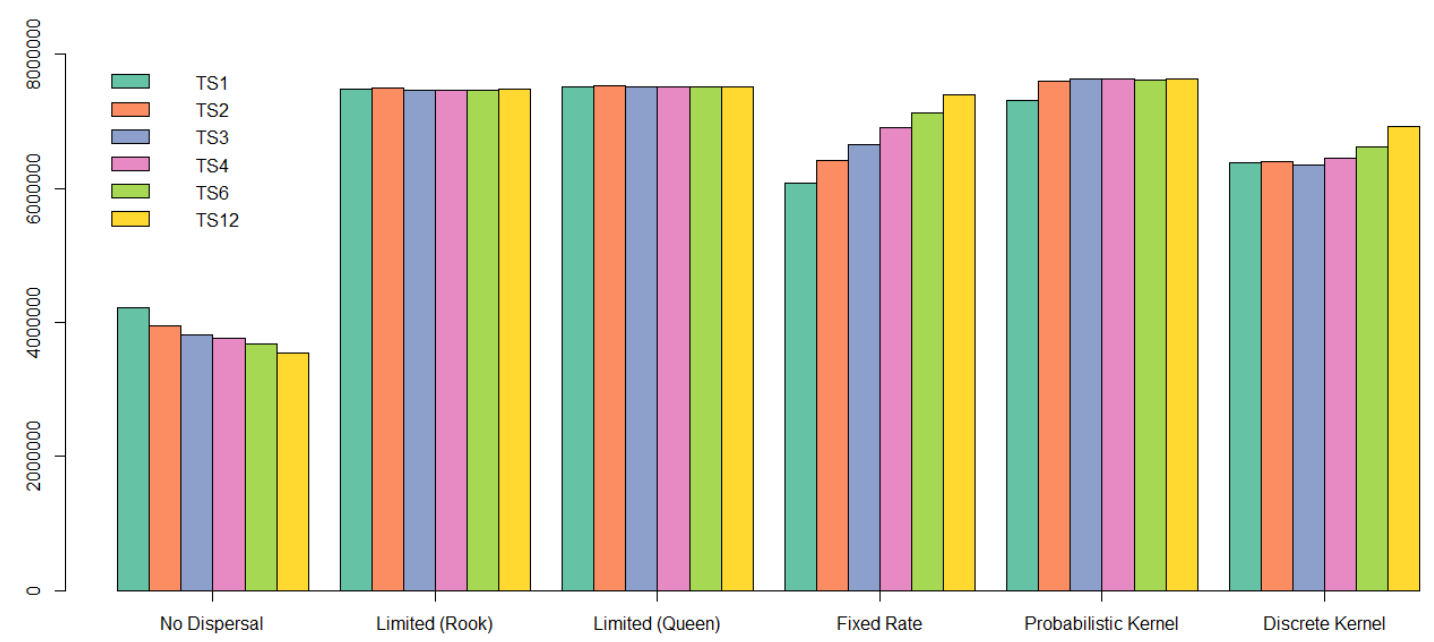

Figure 3.5: Area $\left(\mathrm{km}^{2}\right)$ predicted as present averaged across 29 genera for dispersal models and time-step implementations. 


\section{DisCUSSION}

In this study the genera-environment relationships calibrated with paleovegetation data from $12 \mathrm{ka} \mathrm{BP}$ and projected to the current time period were assessed in an attempt to investigate differences in the accuracy and uncertainty of future projections caused by dispersal modelling. The use of past data to fit species-environment relationships is becoming an integral part of uncertainty research in SDM, as it can allow a measure of accuracy alongside any discussion of uncertainty, with researchers beginning to employ this concept more readily (Araújo et al. 2005; Veloz et al. 2012). However, the paucity of such databases with which to apply accuracy measures has meant studies have had to focus on relatively short time-frames (e.g. 20 years - Araújo et al. 2005). By using paleo-vegetation data from $12 \mathrm{ka}$ BP, dispersal was simulated across a much longer temporal time-period than has previously been used to evaluate dispersal models.

Despite the possibilities, calibrating SDMs on past data from thousands of years ago does introduce added uncertainty into the modelling process. Factors such as the modelled climate scenarios, non-analog climates, the use of fossilized pollen, and the potential niche-differentiation among species of the same genus all introduced uncertainty into the results. Subsequently expectations of accuracy were lower than they would have been for other modelling scenarios. The Neotoma database has been reliably used to establish the paleo-geographic ranges of many North American species (Williams et al. 2004; Blois et al. 2013). From here, data from $12 \mathrm{ka}$ BP was extracted in order to calibrate the models rather than any observations established earlier than this. In part this was due to the fact that Veloz et al. (2012) found poor accuracy for species whose niches had shifted to non-analog climates, and the fact there were 281 genus-level observations for $12 \mathrm{ka} \mathrm{BP}$ rather than only the 28 observations that were available for $21 \mathrm{ka} \mathrm{BP}$. Overall 
a relatively good predictive accuracy was recorded when models were projected into current (12ka BP) and future time (0ka BP) (Table 3.1).

MaxEnt predicted the $12 \mathrm{ka}$ BP distribution well but decreased in accuracy for the 0ka BP distribution, which supported the findings of Veloz et al. (2012). Interestingly however, the contrary relationship was identified for GLM and RF, with the future models improving in terms of accuracy when projected with unlimited dispersal to $0 \mathrm{ka}$ BP (although the differences are not as extensive as MaxEnt). This finding is most likely a construct of the fact that the $0 \mathrm{ka} \mathrm{BP}$ data is validated with a more comprehensive test dataset (i.e. the whole continent), while the $12 \mathrm{ka}$ BP data is validated with a relatively smaller test dataset (i.e. $20 \%$ of the observations or 56 points). The guidelines for evaluating future projections are non-existent, due to the fact that test data for future periods are rare. When it is present, a unique scenario exists whereby accuracy assessment can be undertaken with data covering the entire spatial extent. While only small differences between using the whole continent and 1000 randomly generated points were found (Table 3.1), research should be directed towards better understanding the impacts of using test data from different time periods and differing spatial extents in model evaluation.

The dispersal models differed significantly from each other both in terms of accuracy and area. The accuracy varied most substantially among models when dispersal was simulated as multiple time-steps, with the variation increasing as the number of timesteps increased (Figure 3.3). Surprisingly, unlimited dispersal (UD) and limited dispersal with an eight-neighbor connectivity (L2) had identical accuracies and areas (Figure 3.3, Figure 3.4). Limited dispersal methods are often considered as a pessimistic case of dispersal, but for species which have continuous distributions at a relatively coarse spatial resolution, they can report similar results to unlimited dispersal (Holloway et al. 2016). 
No dispersal (XD) and fixed rate dispersal (FR) actually resulted in higher upper-quartile scores of TSS than the other methods, suggesting that these models actually better predicted the distribution of some species. However, TSS rewards both sensitivity and specificity, and the higher scores for XD and FR are due to a higher specificity score for relatively sparse species, with reasons for this pertaining to an uneven number of absence points in the test dataset compared to presence points.

As an illustration, the suitable abiotic conditions for each 1,000 year interval for Abies are presented in Figure 3.6. It can be seen that the area predicted to be abiotically suitable is much smaller for $4 \mathrm{ka}$ and 8ka BP, corresponding with the drop in median TSS score for L1 (Figure 3.3). Simulating dispersal from a smaller geographic area subsequently results in less overlap area for the L1 model. This example highlights how different the abiotic suitability can be across a long temporal period, and how a simple difference of simulating dispersal from either $4 \mathrm{ka}$ or $3 \mathrm{ka}$ BP can alter the accuracy and the area of a projection.

As expected, unlimited dispersal projected the largest area as present while no dispersal projected the smallest area as present (Table 3.4). All the dispersal models differed significantly from unlimited dispersal with the exception of the discrete dispersal kernels (Table 3.4). This result is congruent with other studies conducted (Holloway et al. 2016), in part due to the fact that the discrete dispersal kernel identifies the maximum possible dispersal distance and treats all locations within this distance as equally accessible. While this may not be the most representative method of long-distance dispersal, it consistently results in higher accuracies compared to the other methods. The importance of long-distance dispersal (LDD) events is well-noted in dispersal ecology (Nathan et al. 2008b), and as such, models should continue to find ways of incorporating LDD, otherwise they will persist at under-predicting accessible locations. 


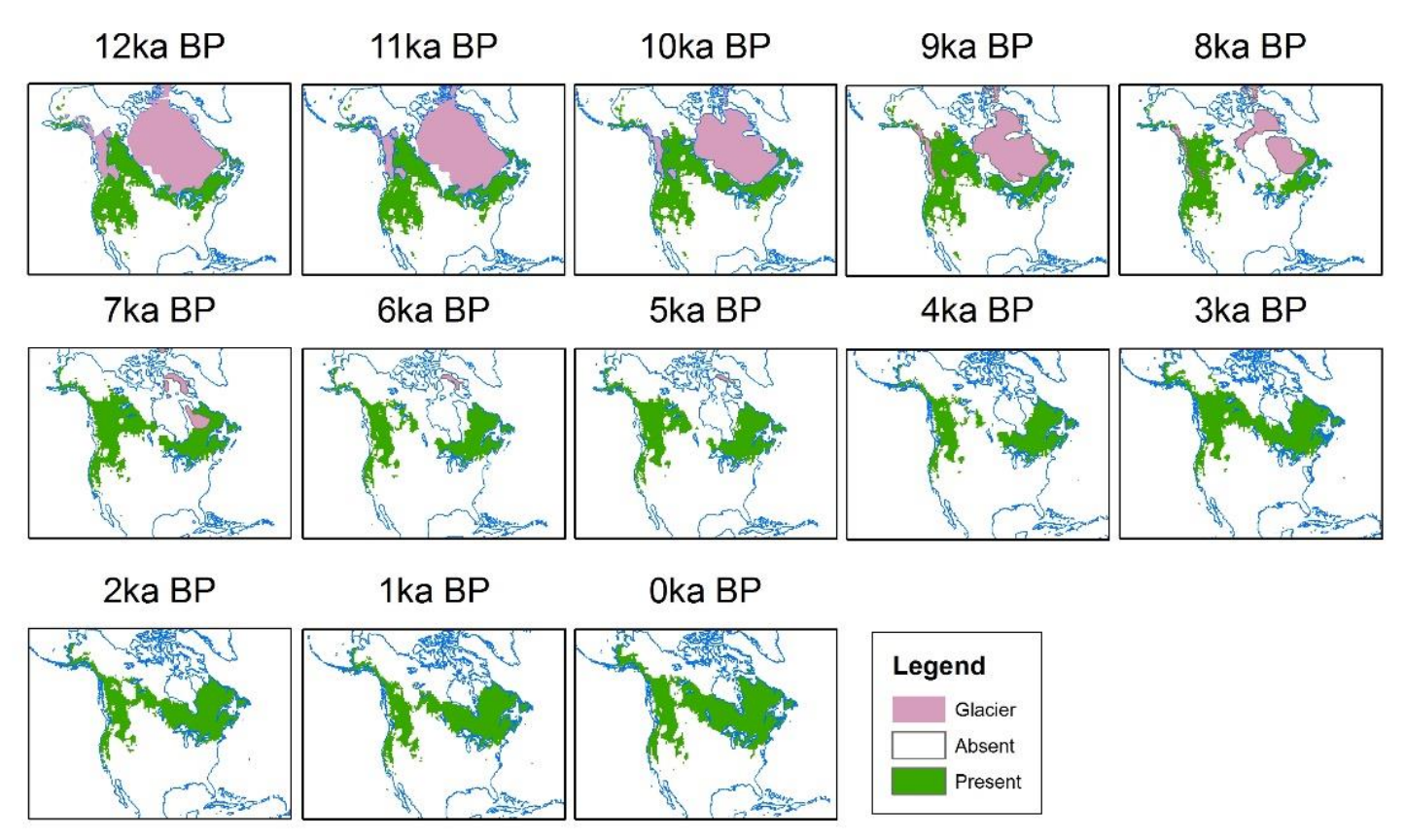

Figure 3.6: Abiotic suitability of Abies projected at 1,000 year intervals.

For the fixed rate (FR) and the discrete dispersal kernel (DK) models, the area predicted as present substantially increased as the number of time-steps simulated in the dispersal models also increased (Figure 3.4, 3.5; Table 3.4). This pattern generally held true for all models of dispersal, albeit the areal differences were not so large (Table 3.4). However, these findings are contrary to the general consensus that when modelled in multiple stages, the future projections could be further constrained by any unfavorable intermediate abiotic conditions which prevent the species from spreading across the entire study area (Figure 3.6). These findings are a result of the spatial scale (resolution) of the digital conceptualizations of these phenomena. SDMs were calibrated at a spatial resolution of $50 \mathrm{~km}$ (to match the spatial resolution of the climate data) across the continent of North America. For SDMs fit at a continental extent, a coarse resolution is considered appropriate, as it does well to capture the trade-off between the resolution and 
extent (Elith and Leathwick 2009). The mean dispersal rate of genera with a dispersal mode of winged seeds (pterometeorochory) is $117 \mathrm{~m}$ every 18 years (Table 3.2). Therefore, when simulated as a one-step process over 12,000 years, the fixed rate of dispersal is equivalent to $(117 *(12000 / 18))$ which is $78,000 \mathrm{~m}$ or 2 grid cells at a $50 \mathrm{~km}$ resolution. For the twelve step simulation, the fixed rate of dispersal for 1,000 years is equivalent to $(117 *(1000 / 18))$ which is $6,500 \mathrm{~m}$ or 1 grid cell at a $50 \mathrm{~km}$ resolution. Due to the inherent assumption in SDM (and the wider GIScience concept of a raster) that each grid cell is homogenous, once a population is deemed to have 'colonized' a grid cell, then the entire grid cell is modelled as present. Therefore, when dispersal is modelled as a twelve-step process and continuous abiotic suitability every 1,000 years is assumed, then the genera will disperse one grid cell every time-step, equivalent to $600 \mathrm{~km}$ or 12 grid cells. This is six times the distance compared to the one time-step model. Future research using this methodology needs to be aware of the scale issues, especially if dispersal distance is substantially lower than the resolution used.

Many dispersal kernels are theoretical and are based on little or no empirical data. By following Vittoz and Engler's (2007) methodology, a database on dispersal distances for North American flora was compiled, and these values were used to create realistic dispersal kernels. The generation of these kernels should be beneficial for future studies working in this region and serve as a platform with which to improve our understanding of seed dispersal. The decision to incorporate observational and anecdotal information in the kernels was due to the simple fact that otherwise the database would be underrepresented. Finally, seed dispersal can be the result of multiple dispersal vectors (e.g. Quercus spp are often dispersed by both gravity (autochory) and by small mammals and birds stocking acorns (dyszoochory)). It was decided that only one method for each genus would be modelled in order to improve interpretation of the results. However, kernels 
could be combined to better represent the multiple dispersal agents, although no methodology currently does this in a succinct and realistic manner.

\section{CONCLUSION}

The choice of dispersal model in SDM can substantially influence the accuracy and uncertainty of future geographic range projections. No dispersal (XD) and fixed rate (FR) methods under-predicted accessibility, while the discrete dispersal kernel (DK) resulted in almost identical results to unlimited dispersal (UD). In particular, the comparisons among dispersal simulated as multiple time-steps suggests that this is another user-decision that could considerably alter projections and in-turn inferences made of the sustainability of many species under climate-change. Principally, the assumption of homogeneity in abiotic suitability of locations between current and future time should be revisited. Substantial differences were found in abiotic suitability across intermediate time-periods, which resulted in differences of accuracy based on the abiotic suitability of the final dispersal step. The empirically derived dispersal distances were relatively small compared to the resolution of the SDM that resulted in simulated dispersal distances much greater than expected. How scale influences these dispersal models should also be a priority for SDM researchers. Finally, models which accounted for long-distance dispersal events (e.g. DK) resulted in higher accuracy despite their relatively unrealistic implementation. Future research should therefore strive to better model the long-distance dispersal events. Dispersal modelling should now be a compulsory aspect of any SDM extrapolating species-environment relationships across time or space, and this study has added a timely addition to the growing literature surrounding the uncertainty among model selection. Following this investigation of the dispersal models, the next stage of this research will be to evaluate the ecological 
processes causing range shifts, and in particular whether incorporating a measure of dispersal alongside abiotic and biotic factors advances the understanding of how species have responded to changing climates since the Quaternary. 


\section{CHAPTER 4: UNCERTAINTY ANALYSIS OF STEP-SELECTION FUNCTIONS: THE EFFECT OF MODEL PARAMETERS ON INFERENCES ABOUT THE RELATIONSHIP BETWEEN ANIMAL MOVEMENT AND THE ENVIRONMENT2}

\section{INTRODUCTION}

Step-selection function (SSF) is a powerful new spatial modeling approach that has been developed as an extension of resource-selection function (RSF - a function that is proportional to the probability of the use of a resource unit by an organism - Manly et al. 2002), and is beginning to gain traction as an important tool for studying conservation issues associated with animal movement. SSF was developed by Fortin et al. (2005) by combining several methods in order to improve the ability to model resource availability in a home range. These concepts improved upon previous models that did not limit resource availability to an accessible distance of current animal location (Forester et al. 2009). Whereas RSF considers the location of an observation, SSF considers the step between two locations. The observed step between two successive locations is compared to a number of alternatively generated steps that the animal could have taken (Figure 4.1), and the coefficients from the case-control regression identify which environmental variables characterize the movement steps actually taken. The majority of studies have estimated SSF in the form of:

$$
\widehat{w}(x)=\exp \left(\beta_{1} x_{1}+\beta_{2} x_{2}+\ldots+\beta_{n} x_{n}\right)
$$

where $\beta_{n}$ is the coefficient estimated by the conditional logistic regression for the variable $x_{n}$. Steps with higher SSF scores $\widehat{w}(x)$ have a higher likelihood of being chosen

\footnotetext{
2 The Version of Record (VoR) of this manuscript has been published and available in Lecture Notes in Computer Science (2014) http://springer.com/ 10.1007/978-3-319-11593-1_4. Amendments to the VoR have been made. PH was lead author on this article, developing the research question, methodology, analysis and results. As such there is no conflict for the content of this manuscript to constitute part of this dissertation.
} 
by the animal, meaning that SSF can help to identify the influence of the environment on animal movements by revealing where they are most likely to be at the end of a movement step.

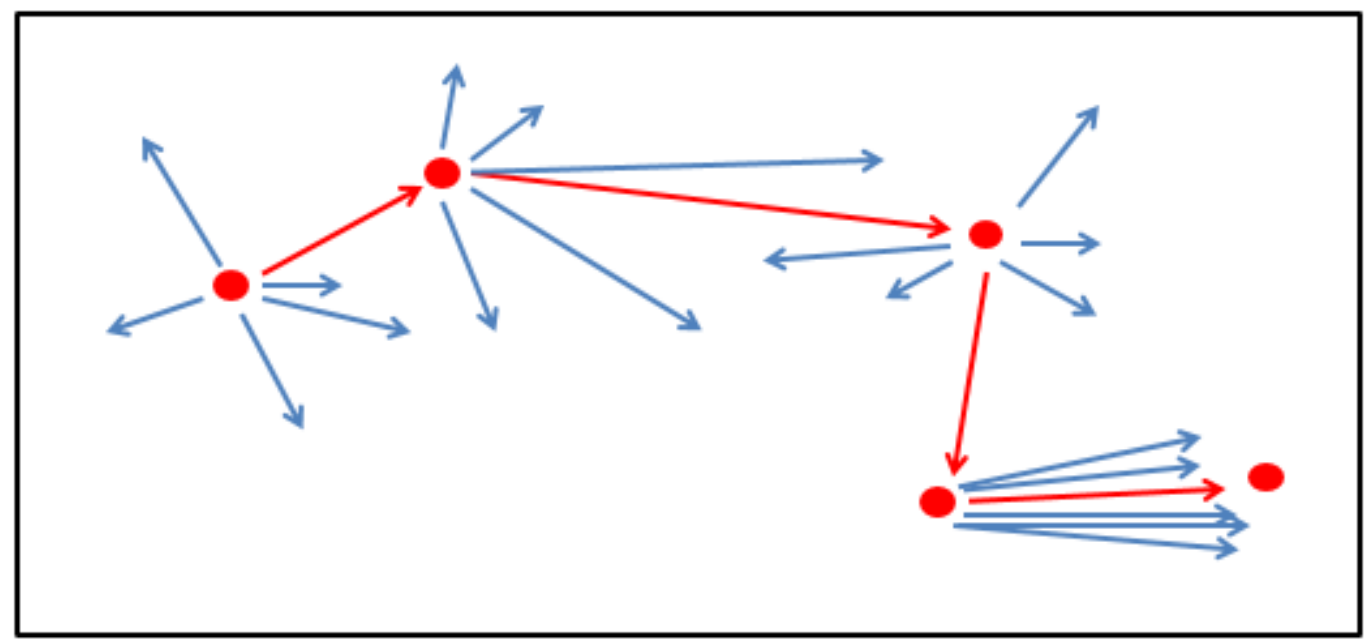

Figure 4.1: Conceptual depiction of step-selection function. Red dots represent successive telemetry locations of an individual, with red arrows representing observed steps. The blue arrows represent the available steps that the individual could have taken.

Since its development, SSF has been used to study a wide range of species, from wrens (Gillies et al. 2011) to wolves (Latham et al. 2011), and is increasing in its popularity among ecologists due to the power of SSF to identify the influence of environmental variables on the movement of animals. Subsequently, SSF was identified as a method which could be utilized to explore and investigate how regular movement could be incorporated within the species distribution modelling framework. However, fitting an SSF involves a number of user-decisions, and it is unknown how these decisions influence the modelling procedure. Table 4.1 provides a summary of previous SSF studies, highlighting the user-decisions made by researchers during the model building phases. Specific to this dissertation, a number of questions arose regarding the 
uncertainty of user-decisions when fitting an SSF to address regular movement. These pertained to environmental variables, inferences of animal movement across the landscape and the processing time that would be required to conduct complex spatial simulations based on SSF. Therefore, this chapter addresses what I considered to be some of the most important and subjective decisions in SSF. Most of these user-decisions were also described as being potentially important by an SSF review paper that was published at a similar time to this research (Thurfjell et al. 2014). With SSF currently being used to inform researchers and conservationists about the relationship between animal movement and the environment, the uncertainty associated with the model building phases needs to be explored so that any applications and interpretations can be made with as much confidence as possible.

\section{Methods of Generating Available Steps}

As SSFs compare used versus available steps, the method used to generate the available steps could potentially have the most influence on the results (Thurfjell et al. 2014). The method introduced by Fortin et al. (2005) is still the most commonly applied and involves generating available steps from an empirical distribution of movement parameters (turn angle and step length) from other monitored individuals. Empirical distributions are classified in frequency tables of varying interval size, and draws are then made based on a rejection algorithm, whereby an interval is randomly selected from the table alongside a random number from a uniform distribution. If the random number is less than or equal to the specified probability value, a value from that interval is returned (Beyer 2012). Fortin et al. (2005) used intervals of $20^{\circ}$ and $50 \mathrm{~m}$ for the frequency tables for turn angle and step length respectively which equated to 18 bins $\left(360^{\circ} / 20^{\circ}\right)$ for turn angles and 60 bins (maximum length of $3000 \mathrm{~m} / 50 \mathrm{~m}$ ) for step lengths. In theory, the sm- 
Table 4.1: User-decisions in recently published step-selection function studies.

\begin{tabular}{|c|c|c|c|c|c|c|}
\hline Species & $\begin{array}{l}\text { Number of } \\
\text { Individuals }\end{array}$ & $\begin{array}{c}\text { Number of } \\
\text { Alternative } \\
\text { Steps }\end{array}$ & $\begin{array}{l}\text { Temporal } \\
\text { Resolution }\end{array}$ & $\begin{array}{c}\text { Modelling Approach } \\
\text { of Conditional } \\
\text { Logistic Regression }\end{array}$ & Method to Generate Alternative Steps & Reference \\
\hline Elk & 13 & $200 *$ & 5 hours & Population & $\begin{array}{l}\text { Drawn from Empirical Distribution } \\
\text { (equal bins of } 20^{\circ} \text { and } 50 \mathrm{~m} \text { for TA and } \\
\text { SL) }\end{array}$ & Fortin et al. 2005 \\
\hline Roe Deer & 20 & 10 & $\begin{array}{c}2 \text { hours and } 6 \\
\text { hours }\end{array}$ & Population & $\begin{array}{c}\text { Drawn from Empirical Distribution } \\
\text { (equal bins of } 20^{\circ} \text { and } 50 \mathrm{~m} \text { for TA and } \\
\mathrm{SL} \text { ) }\end{array}$ & $\begin{array}{l}\text { Coulon et al. } \\
\quad 2008\end{array}$ \\
\hline Moose & 64 & 2 & 2 hours & Population & $\begin{array}{l}\text { Drawn from Random Distribution for } \\
\text { TA and a Quartile Distribution (99\%) } \\
\text { for SL }\end{array}$ & $\begin{array}{l}\text { Bjorneraas et al. } \\
2011\end{array}$ \\
\hline $\begin{array}{l}\text { Antshrike } \\
\text { and Wren }\end{array}$ & 30 & 20 & 15 minutes & Population & $\begin{array}{l}\text { Drawn from Distribution of similar TA } \\
\text { and SL (no further explanation) }\end{array}$ & Gillies et al. 2011 \\
\hline Wolves & 31 & 25 & 2 hours & Population & $\begin{array}{c}\text { Drawn from Empirical Distribution } \\
\text { (equal bins of } 20^{\circ} \text { and } 200 \mathrm{~m} \text { for TA and } \\
\text { SL) }\end{array}$ & $\begin{array}{l}\text { Latham et al. } \\
\quad 2011\end{array}$ \\
\hline $\begin{array}{l}\text { Pine } \\
\text { Martins }\end{array}$ & 8 & $\begin{array}{c}\text { No } \\
\text { mention }\end{array}$ & Uneven & Population & Generated random paths & $\begin{array}{l}\text { Pereboom et al. } \\
2008\end{array}$ \\
\hline $\begin{array}{l}\text { Grizzly } \\
\text { Bears }\end{array}$ & 7 & 20 & 1 hour & Individual & $\begin{array}{l}\text { Drawn from movement paths to } \\
\text { distinguish activity from resting }\end{array}$ & $\begin{array}{l}\text { Northrup et al. } \\
2012\end{array}$ \\
\hline Lynx & 64 & 5 & 30 minutes & Individual & Drawn from a Random Distribution & $\begin{array}{l}\text { Squires et al. } \\
2013\end{array}$ \\
\hline Caribou & 49 & 10 & 4 hours & Population & $\begin{array}{l}\text { Drawn from an Empirical Distribution } \\
\text { (no mention of TA or SL bin sizes) }\end{array}$ & $\begin{array}{l}\text { Beauchesne } \text { et al. } \\
2013\end{array}$ \\
\hline $\begin{array}{l}\text { Grizzly } \\
\text { Bears }\end{array}$ & 27 & 20 & 4 hours & Population & $\begin{array}{l}\text { Random selection of alternative steps } \\
\text { (no further explanation) }\end{array}$ & $\begin{array}{c}\text { Roever } \text { et al. } \\
2010\end{array}$ \\
\hline Bison & 100 & 2 & 5 minutes & Population & $\begin{array}{l}\text { Drawn from an Empirical Distribution } \\
\text { (with each new step, SL and TA were } \\
\text { added to the distribution) }\end{array}$ & $\begin{array}{l}\text { Courant and } \\
\text { Fortin } 2012\end{array}$ \\
\hline
\end{tabular}


aller the intervals of the bins, the closer the empirical distribution will match a more continuous real distribution. Other SSF studies have followed, using evenly spaced intervals for turn angle and step length (Coulon et al. 2008; Latham et al. 2011). However, the intervals do not need to be even, and in portions of the distribution where the density is constant, a wider interval may be beneficial compared to when density changes rapidly and narrower bins may be favorable (Beyer 2012); although, this adaptive method has yet to be employed.

Other studies have generated the available steps using a random distribution (Squires et al. 2013), a quantile distribution (Bjørneraas et al. 2011), and a parametric distribution (Forester et al. 2009). When a random distribution has been used, turn angle was randomly drawn from between $-180^{\circ}$ to $+180^{\circ}$, and step length was randomly drawn from any value between 0 and the maximum step length. The same method has been used for quantile distributions, but the values were capped at a specific percentage of the distribution. Bjørneraas et al. (2011) used the 99\% quantile for step lengths, although this method was not used to select turn angle as the distribution is in degrees and so cannot be capped in the same manner.

A recent study by Forester et al. (2009) examined the use of SSFs using three methods of generating available samples (in terms of step length); random (termed uniform), empirical and parametric and compared the results on simulated data and elk data in Yellowstone National Park. They found that empirical and parametric distributions performed better than the uniform distribution which was the method furthest from resembling a continuous real distribution. The level of bias in the estimates differed between empirical and parametric distributions and was dependent on the size of the coefficient. With empirical distributions currently more widely used in SSF studies, 
and with the uncertainty between using even and uneven intervals, this will be the main focus of this section.

\section{Number of Steps}

Once the available steps have been generated, the next user-decision is the number of available steps used in the comparison. In their seminal paper, Fortin et al. (2005) used 200 available steps, mainly because their research question was directed at rare habitat selection, although they noted that future SSF studies would not need such a high number. This remains the largest number of steps used in SSF studies so far, with the next highest value only 25 steps (Latham et al. 2011), while the minimum is two (Bjørneraas et al. 2011). Subsequent studies have not specified the reasoning behind their selection of fewer steps beyond citing the statement of Fortin et al. (2005) that they would not need 200. RSFs have been used more extensively than SSFs, and while some studies have suggested using 10,000 available locations (in total) in the comparison (Lele and Keim 2006; Barbet-Massin et al. 2012), using an ad hoc approach still appears to be the norm (Northrup et al. 2013). Subsequently, it is unknown whether a high number of available locations/steps over-samples the environmental choices available, or whether a low number of available locations/steps under-samples.

\section{Modelling Approach}

The majority of SSF studies have used conditional logistic regression as the statistical method, but there has been variation with respect to how the analysis is conducted. Conditional logistic regression in which regression parameters are fit for all of the individuals together has been the most widely used approach. An alternative to this has recently been employed whereby model parameters are fit for each individual separately and then averaged to attain aggregate information, and has been termed 
individual modelling (Sawyer et al. 2006; Northrup et al. 2012; Squires et al. 2013). Individual modelling can potentially capture more individual movement traits while still being applicable to the aggregation of individuals and is beginning to be used more regularly.

\section{Individual Variation}

The effect of individual variation on results has only recently begun to be incorporated in SSF studies. Due to the high costs of GPS units, sample sizes are often small to moderate, with several SSF studies containing less than 20 individuals (Fortin et al. 2005; Pereboom et al. 2008; Northrup et al. 2012). In studies where sample sizes are lower, individual traits may have more influence on the results, although in a recent study using seven Eleonora's falcons, Gschweng et al. (2012) found that removing one from the study did not result in a significant change in habitat use. Few studies have taken into account the idiosyncratic differences among individual animals in SSF models and this is an area that needs further study.

\section{Uncertainty Analysis}

Uncertainty analysis allows researchers to assess the range of outputs associated with the model responses as a result of variations in parameter values used in the model input (Crosetto and Tarantola 2001). This uncertainty analysis will provide SSF practitioners with insight into how to choose appropriate model parameters as well as information on the level of uncertainty associated with the results. More specifically, this analysis will directly inform decisions undertaken in the remaining two research chapters of this dissertation. Therefore, this uncertainty analysis will analyze the effect of 1) generating the available steps based on a) turn angle and b) step length, 2) the number of 
available steps used, 3) the modelling approach used, and 4) the number of individuals used.

\section{METHODOLOGY}

\section{Data Collection}

Oilbird (Steatornis caripensis) data was obtained from Holland et al. (2009) via Movebank (2013). GPS with remote UHF readout was used to collect locations of four individuals with ten minute intervals, resulting in approximately 800 fixes for use, with the number of observations ranging from 133 to 264. Brown hyena (Hyaena brunnea) data was collected by Maude (2010) in the Makgadikgadi Pans region of northern Botswana between June 2004 and December 2007 with the support of the Makgadikgadi Brown Hyena Project. GPS locations were recorded for each hyena at 1 hour intervals. Ten hyenas were used in this analysis across a time period of a month, which resulted in 4000 fixes for use, ranging from 278-432. The environmental variables used in both of these analyses are briefly described in table 4.2 .

\section{Uncertainty Analysis: Generating Available Steps}

Steps were generated from drawing from four distributions for both turn angle and step length; empirical distribution (even bins), empirical distribution (uneven bins), random distribution, and quantile distribution (99\%) (n.b. quantile distribution was not used for turn angle). Empirical distributions of even bins formed intervals of $20^{\circ}$ for turn angles, and $187.3 \mathrm{~m}$ and $362 \mathrm{~m}$ for step length for oilbirds and hyenas respectively. The values of step lengths equates to $2 \%$ of the maximum step length. Empirical distributions of uneven bins formed intervals of 5.56\% quantiles for turn angle and $2 \%$ quantiles for step lengths. These values ensured that the same number of bins would be used for even and uneven empirical distributions. 


\section{Uncertainty Analysis: Number of Steps}

The number of available steps was chosen to fall between the minimum (2) and the maximum value used (200) in previous SSF studies: 2, 10, 20, 50, 100, and 200.

\section{Uncertainty Analysis: Modelling Approach}

Conditional logistic regression (equation 4.1) was done in $\mathrm{R}$ using the survival package (Therneau 2013). This was either conducted with all individuals in one dataframe (herein referred to as aggregate modelling), or for each individual separately with the coefficients averaged to generate an aggregate level model (herein referred to as individual modelling).

\section{Uncertainty Analysis: Individual Variation}

Conditional logistic regression was fit for all the individuals, while systematically dropping one individual from the analysis (n.b. this analysis was undertaken using available steps generated from the empirical distribution of all the individuals). All combinations of turn angle, step length, number of steps and modelling approach was also conducted for each model without certain individual.

\section{Data Analysis}

In total, 720 oilbird and 1584 hyena regression models were fit. The coefficient values were compared for each variable with Wilcoxon matched pairs signed rank test using a Bonferroni corrected $\alpha$ of 0.05 according to the number of comparisons made. This test converts scores to ranks and compares them across the two conditions. The effect size of the test was calculated by dividing the $z$ value by the square root on $N$ and using the Cohen (1988) criteria of $0.1=$ small effect, $0.3=$ medium effect and $0.5=$ large effect. 
Table 4.2: Information about the environmental variables used in the regression models. The IGBP classification scheme was used for the MODIS land cover product (LP DAAC 2013), which delineates into 16 classes.

\begin{tabular}{|c|c|c|c|c|}
\hline Animal & $\begin{array}{l}\text { Environmental } \\
\text { Variable }\end{array}$ & Method & Hypothesis & Source \\
\hline Oilbird & $\begin{array}{l}\text { Land Cover - } \\
\text { Evergreen Broadleaf } \\
\text { Forest }\end{array}$ & $\begin{array}{l}\text { Categorical Variable - Value of } \\
\text { land cover at the end of the step }\end{array}$ & $\begin{array}{l}\text { Oilbirds eat the fruit of tropical laurels. The } \\
\text { majority of these trees are evergreen broadleaf } \\
\text { species. The oilbirds are more likely to move } \\
\text { through land covers which contain food sources. }\end{array}$ & $\begin{array}{l}\text { LP DAAC } \\
(2010)\end{array}$ \\
\hline Oilbird & $\begin{array}{l}\text { Cropland - } \\
\text { Percentage of } \\
\text { cropland }\end{array}$ & $\begin{array}{l}\text { Continuous Variable - Value of } \\
\text { cropland at the end of the step }\end{array}$ & $\begin{array}{l}\text { Oilbirds also eat the fruit of oil palms, a } \\
\text { commercial crop in South America. The higher the } \\
\text { percentage of cropland, the higher chance of oil } \\
\text { palms in the area. }\end{array}$ & $\begin{array}{l}\text { Ramankutty } \\
\text { et al. } \\
(2010)\end{array}$ \\
\hline Oilbird & Distance to Roads & $\begin{array}{l}\text { Continuous Variable - If step } \\
\text { crosses line }- \text { distance }=0 . \\
\text { Else, distance equals the } \\
\text { average distance from start, mid } \\
\text { and end of the step }\end{array}$ & $\begin{array}{l}\text { Birds have been found to frequent edge habitats, } \\
\text { with roads one of the most common features } \\
\text { splitting habitats. }\end{array}$ & $\begin{array}{l}\text { CIESIN } \\
(2013)\end{array}$ \\
\hline Hyena & $\begin{array}{l}\text { Land Cover - } \\
\text { Savanna }\end{array}$ & $\begin{array}{l}\text { Categorical Variable - Value of } \\
\text { land cover at the end of the step }\end{array}$ & $\begin{array}{l}\text { Hyenas may visit savanna habitats more often due } \\
\text { to a higher potential of prey species. }\end{array}$ & $\begin{array}{l}\text { LP DAAC } \\
(2010)\end{array}$ \\
\hline Hyena & $\begin{array}{l}\text { Land Cover - Open } \\
\text { Shrublands }\end{array}$ & $\begin{array}{l}\text { Categorical Variable - Value of } \\
\text { land cover at the end of the step }\end{array}$ & $\begin{array}{l}\text { Hyenas may visit shrubland habitats more often } \\
\text { due to a higher potential of prey species. }\end{array}$ & $\begin{array}{l}\text { LP DAAC } \\
(2010)\end{array}$ \\
\hline Hyena & $\begin{array}{l}\text { Land Cover - } \\
\text { Fragmentation of } \\
\text { habitat }\end{array}$ & $\begin{array}{l}\text { Continuous Variable - Number } \\
\text { of surrounding habitats of the } \\
\text { cover at the end of the step }\end{array}$ & $\begin{array}{l}\text { The more surrounding habitats results in the } \\
\text { potential of more prey species. More habitats may } \\
\text { mean more species }\end{array}$ & $\begin{array}{l}\text { LP DAAC } \\
(2010)\end{array}$ \\
\hline Hyena & Distance to Roads & $\begin{array}{l}\text { Continuous Variable - If step } \\
\text { crosses line }- \text { distance }=0 . \\
\text { Else, distance equals the } \\
\text { average distance from start, mid } \\
\text { and end of the step }\end{array}$ & $\begin{array}{l}\text { Hyenas could use roads to travel along or obtain } \\
\text { road kill. Alternatively they may avoid them due } \\
\text { to the dangers. }\end{array}$ & $\begin{array}{l}\text { CIESIN } \\
(2013)\end{array}$ \\
\hline
\end{tabular}




\section{Results}

For hyenas (table 4.3) and oilbirds (table 4.4), some combination of coefficients differed for each variable (n.b. tables only contain comparisons where one significant difference exists). The distribution used to generate the turn angle differed between random and empirical (even or uneven), although this effect was very small. Step length distribution caused a variety of significant differences in the coefficients, with Figure 4.3 showing the range of coefficient values for savanna selection for hyenas and evergreen broadleaf forest for oilbirds. The number of available samples used in the model resulted in some small significant differences, although these were not consistent between species (tables 4.3 and 4.4). Individual modelling resulted in inflated coefficient values when compared to the aggregate model (Figure 4.2), with only two hyenas and one oilbird appearing to cause the increased values (Figure 4.4). Finally, systematically removing individuals caused significant differences, with the largest differences appearing to match the individuals causing the inflated coefficients in Figure 4.4.

\section{DISCUSSION}

The objective of this study was to investigate the uncertainty associated with userdecisions and how these decisions influence the results obtained from step-selection functions for two different types of animals (oilbirds and brown hyenas). Oilbirds forage for food by night with most of their food source coming from evergreen laurels, while brown hyenas forage solitarily across large home ranges encompassing a variety of land covers. Both datasets had a considerable number of observations, as well as differing temporal resolutions. Subsequently, any similarities or differences in the results could be attributed to either a user-decision or a species-specific trait, and the results from this analysis should be used by other researchers to help inform their implementation of SSF. 


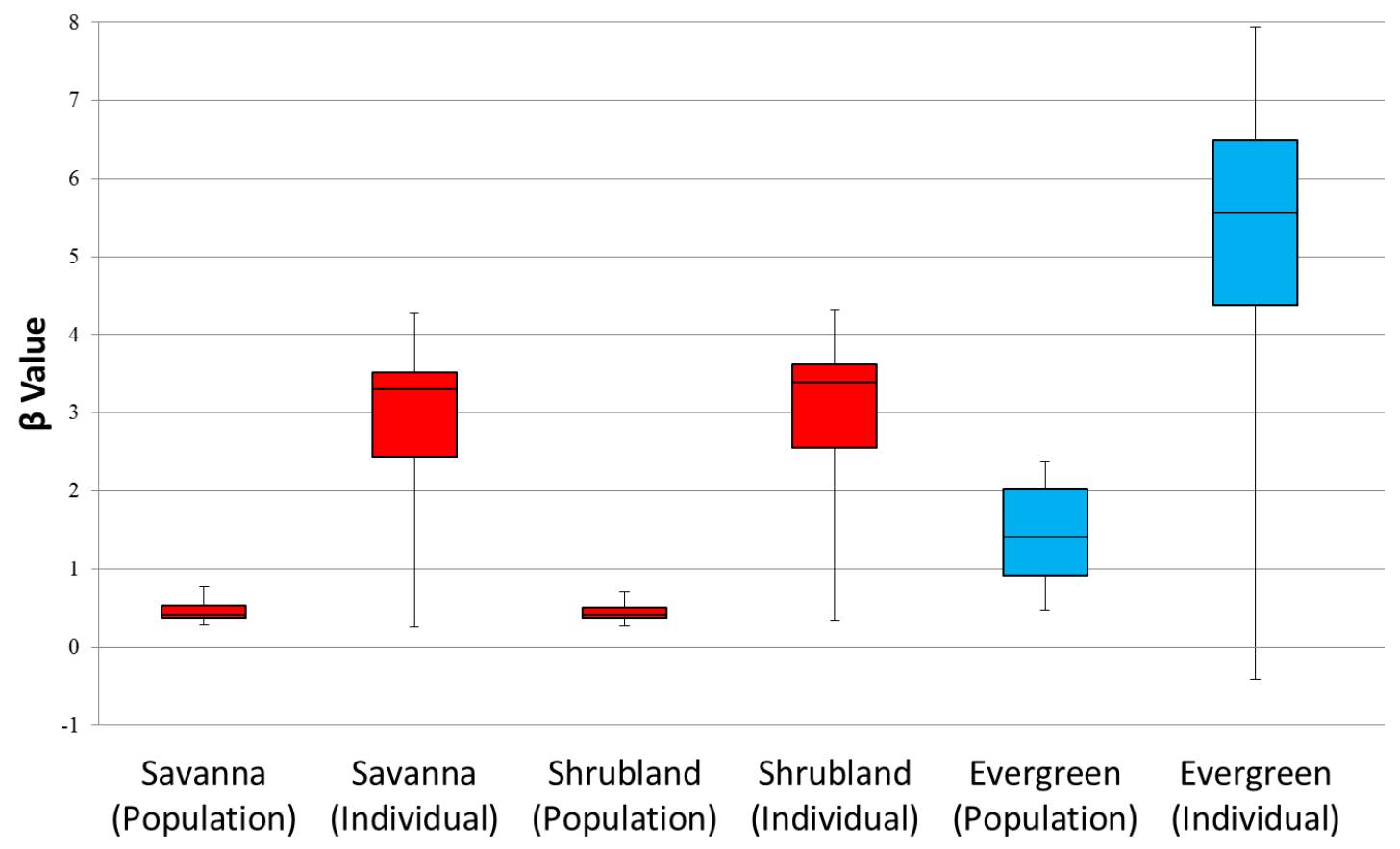

Figure 4.2: $\quad \beta$ values for savanna and shrublands for individual and population modelling of hyena SSF (red) and evergreen broadleaf forest for oilbirds (blue).

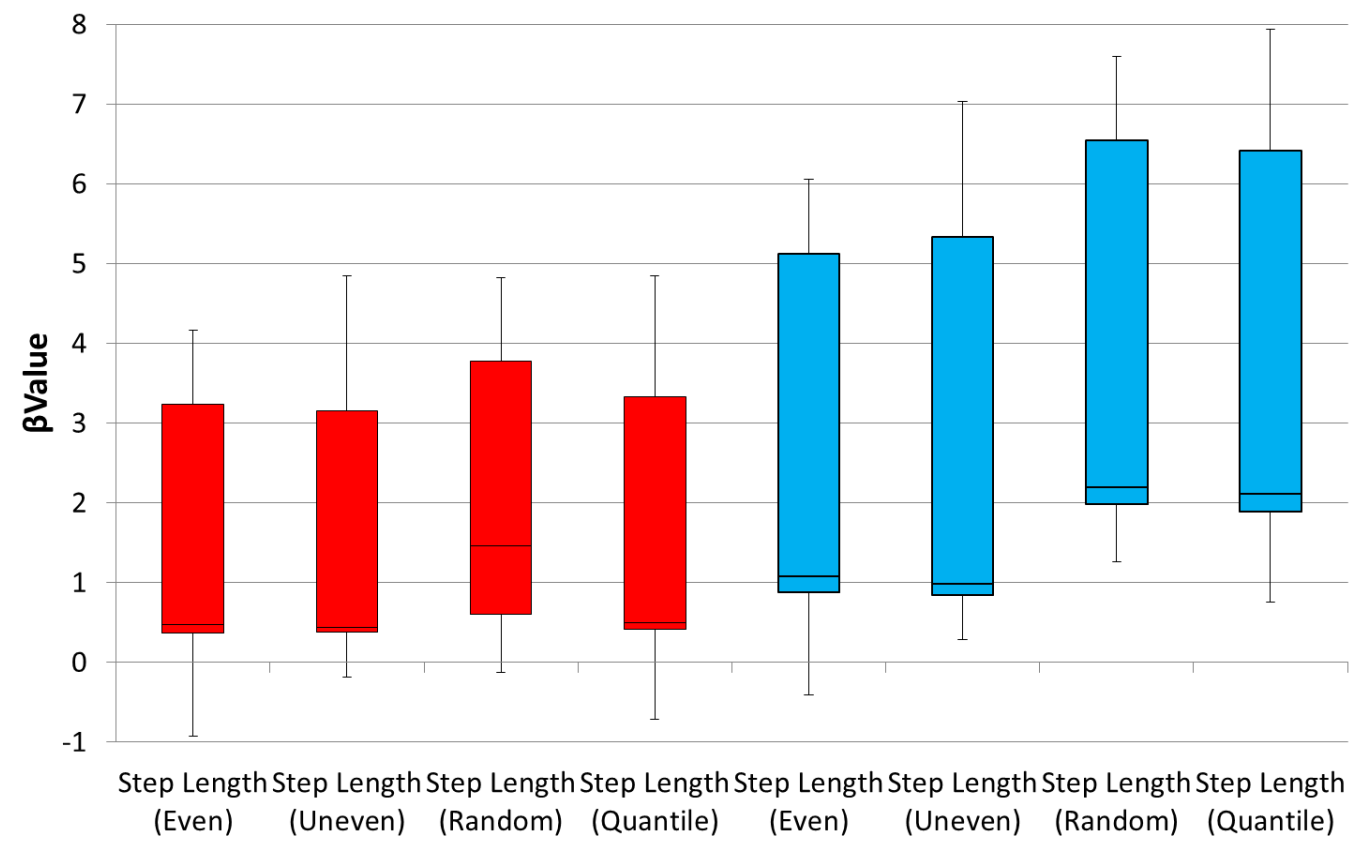

Figure 4.3: $\quad \beta$ values for savanna for hyenas (red) and evergreen broadleaf forest for oilbirds (blue) based on the four methods of generating step lengths. 
Table 4.3: Wilcoxon matched pairs signed rank for hyenas, value refers to second group, *medium effect, $* *$ large effect.

\begin{tabular}{|c|c|c|c|c|c|}
\hline Variable & & $\beta$ Roads & $\beta \mathrm{Sav}$ & $\beta$ Shrub & $\beta$ Edge \\
\hline Method & $\begin{array}{l}\text { Aggregate - } \\
\text { Individ }\end{array}$ & Lower & Higher** & Higher** & Lower** \\
\hline \multirow[t]{3}{*}{ Turn Angle } & Even - Random & n.s. & Lower & Lower & Higher \\
\hline & Even - Uneven & n.s. & n.s. & n.s. & Lower \\
\hline & Uneven - Random & n.s. & Lower & Lower $*$ & Lower * \\
\hline \multirow{6}{*}{$\begin{array}{l}\text { Step } \\
\text { Length }\end{array}$} & Even - Uneven & n.s. & n.s. & Lower & Higher \\
\hline & Even - Random & Lower* & Higher** & Higher** & Higher** \\
\hline & Even - Quantile & n.s. & Higher* & Higher & n.s. \\
\hline & Uneven - Random & Lower* & Higher** & Higher* & Higher* \\
\hline & Uneven - Quantile & n.s. & Higher* & Higher & Lower \\
\hline & Quantile - Random & Lower* & Higher** & Higher** & Higher** \\
\hline \multirow{9}{*}{$\begin{array}{l}\text { Number of } \\
\text { Steps }\end{array}$} & $2-50$ & Lower & n.s. & n.s. & n.s. \\
\hline & $10-20$ & n.s. & Lower* & Lower & n.s. \\
\hline & $10-50$ & n.s. & Lower* & Lower & n.s. \\
\hline & $10-100$ & n.s. & Lower & Lower & n.s. \\
\hline & $10-200$ & n.s. & Lower* & Lower & n.s. \\
\hline & $20-50$ & n.s. & n.s. & Higher & Lower \\
\hline & $20-200$ & n.s. & Lower & n.s. & n.s. \\
\hline & $50-200$ & n.s. & Lower & Lower & n.s. \\
\hline & $100-200$ & n.s. & Lower* & Lower & n.s. \\
\hline \multirow[t]{10}{*}{ Individuals } & All - H1 & Higher & Higher** & Higher** & Lower** \\
\hline & All - H2 & n.s. & Lower & Lower** & Lower** \\
\hline & All - H3 & Higher & Lower* & Higher** & Higher** \\
\hline & All - H4 & n.s. & Higher** & Higher** & Higher** \\
\hline & All - H5 & n.s. & Lower & Lower** & Lower** \\
\hline & All - H6 & n.s. & Lower & Lower** & Lower** \\
\hline & All - H7 & Higher & Lower & Lower* & Higher* \\
\hline & All - H8 & n.s. & Higher* & Higher & n.s. \\
\hline & All - H9 & n.s. & Higher** & Higher** & Lower** \\
\hline & All - H10 & Higher & Lower & Higher & n.s. \\
\hline
\end{tabular}


Table 4.4: Wilcoxon matched pairs signed rank for oilbirds, value refers to second group, *medium effect, $* *$ large effect.

\begin{tabular}{|c|c|c|c|c|}
\hline Variable & & $\beta$ Roads & $\beta$ Evergreen & $\beta$ Croplands \\
\hline Method & Aggregate - Individ & Lower & Higher* & Lower** \\
\hline Turn Angle & Even - Random & n.s. & n.s. & Lower \\
\hline \multirow{6}{*}{$\begin{array}{l}\text { Step } \\
\text { Length }\end{array}$} & Even - Uneven & n.s. & n.s. & Higher \\
\hline & Even - Random & n.s. & Higher** & n.s. \\
\hline & Even - Quantile & n.s. & Higher** & n.s. \\
\hline & Uneven - Random & n.s. & Higher** & n.s. \\
\hline & Uneven - Quantile & n.s. & Higher* & Lower \\
\hline & Quantile - Random & n.s. & Higher* & Higher \\
\hline \multirow{11}{*}{$\begin{array}{l}\text { Number of } \\
\text { Steps }\end{array}$} & $2-10$ & Higher & n.s. & Higher \\
\hline & $2-20$ & Higher & n.s. & Higher \\
\hline & $2-50$ & Higher & n.s. & Higher \\
\hline & $2-100$ & Higher & n.s. & Higher \\
\hline & $2-200$ & Higher & n.s. & Higher \\
\hline & $10-20$ & n.s. & Lower & n.s. \\
\hline & $10-200$ & n.s. & Lower* & n.s. \\
\hline & $20-200$ & n.s. & Lower & n.s. \\
\hline & $50-100$ & n.s. & Lower & n.s. \\
\hline & $50-200$ & n.s. & Lower* & n.s. \\
\hline & $100-200$ & n.s. & Lower* & n.s. \\
\hline \multirow[t]{4}{*}{ Individuals } & All - B1 & n.s. & Higher** & Lower** \\
\hline & All - B2 & Lower & Higher** & Higher** \\
\hline & All - B3 & n.s. & n.s. & Lower \\
\hline & All - B4 & Lower & Higher* & Lower** \\
\hline
\end{tabular}

SSFs associate parameters of movement rules with landscape features, as well as modeling the choices actually presented to the animal as it traverses through the landscape (Chekiewicz et al. 2006). However, if the user-decisions do alter the results observed, then it is difficult to disentangle actual step/habitat preferences from model parameterization decisions. The number of significant differences found in tables 4.2 and 
4.3, and the size of these differences suggests that researchers need to explicitly explore how their definitions of model parameters influence step/habitat preferences.

The largest differences appeared to be between the modelling approach used and systematically removing one individual from the model. The idea behind an individual modelling approach is that the final model will contain results that highlight environmental interactions that better represent individual to population preferences for habitat use, something that is not possible with aggregate modelling (see Figures 4.2, 4.4). Figure 4.2 shows the range of coefficient values for savanna and shrublands (for hyenas) and evergreen forest (for oilbirds), when calculated using the aggregated and individual modelling approaches. Figure 4.4 shows the range of coefficients for each individual hyenas and oilbirds that were averaged in the individual modelling approach. It can be observed that hyenas 3 and 7 and oilbird 4 have much higher coefficients than the others (indicating their increased preference for moving into that specific habitat). The aggregate method appears to suppress the idiosyncratic preferences of these individuals, while the individual method incorporates it with a higher average value. Individual modelling therefore incorporates individual information into the coefficient and researchers need to be aware of these differences, as the method used in analysis could over- or under-estimate the importance of an environmental variable based on the idiosyncratic preferences of just one or two individuals (as shown in this research).

The influence of individuals is furthered highlighted by the differences in results when one individual was dropped from the regression (tables 4.3 and 4.4). Removing either hyena $1,4,8$ or 9 from analysis results in a higher preference for savanna habitats and removing either hyena $1,3,4,8,9$ or 10 from analysis result in a higher preference for shrublands. Similar results are observed for oilbirds (table 4.4). The sample size of hyenas is larger than the sample size used by Gschweng et al. (2012) when they 105 
concluded that removing one individual did not result in statistically different results (albeit the results were not based on conditional logistic regression). Individuals can therefore influence the value of the coefficients obtained, and this should be explored before any statistical model is fit to infer the relationship between animal movement and the underlying environment.

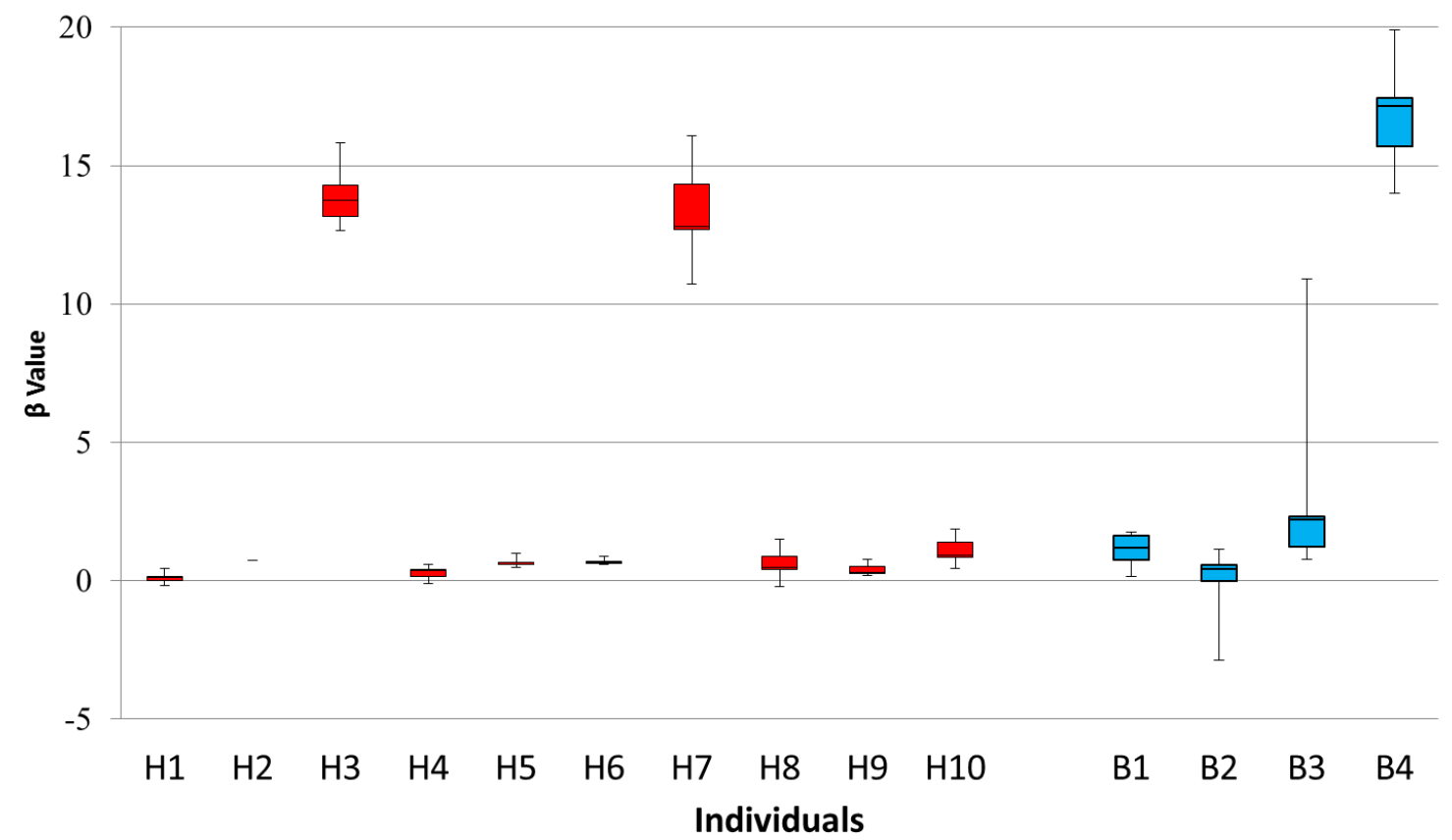

Figure 4.4: $\beta$ values of shrublands for individual hyenas (red) and the $\beta$ values of evergreen broadleaf forest for individual oilbirds (blue).

A recent review outlining the need for an uncertainty analysis of SSF suggested that the method of generating the available steps could be the most important decision when developing this model (Thurfjell et al. 2014). While the method of generating the step length caused significant changes, this was not the case for generating the turn angle (tables 4.3 and 4.4). While significant differences did exist between turn angle distributions, they were either of a small or medium effect for hyenas, and only one 
difference between even and random distributions existed for the coefficient describing the effect of croplands on oilbirds. Step length was a more important variable than turn angle, although it appeared more important for variables where the value was measured at the end of the step (savanna, shrubland, edge habitat for hyenas and broadleaf forest for oilbirds). Distributions which selected randomly up to the maximum (or 99\% quantile) step length resulted in higher coefficients than even or uneven distributions (Figure 4.3). These results could be compounded by infrequent movement steps that occur over great distances, but are not controlled for through their low probability of occurrence as they would be with an empirical distribution. The higher coefficients obtained using a random distribution are a result of longer steps taking the available steps beyond the evergreen forest for oilbirds and the savanna and shrubland for hyenas. This increases the number of alternative habitats the animal could select, and thus increases the coefficient value of their preferred habitat.

Generating steps using a quantile distribution (99\%) resulted in higher coefficients for oilbirds, but not hyenas (Fig. 3). The distribution of step lengths for hyenas is relatively normal (note a slight bimodal distribution), while for oilbirds it is ' $\mathrm{L}$ ' shaped (data not shown). Therefore, using a 99\% quantile distribution removes the most extreme values for the hyenas, but fails to do so with the oilbirds, meaning that the quantile distribution continues to produce results similar to a random distribution. Researchers should investigate the type of distribution associated with step lengths first, before making a decision on the type of distribution they use to generate available steps.

The number of available steps was incorporated in the uncertainty analysis as it is unknown whether a high or low number of available steps over- or under-samples the environmental choices available. While results were not consistent for oilbirds and hyenas, differences in the number of available steps used were found for both (tables 4.3 
and 4.4). Coefficients describing the effects of roads and croplands on oilbird movement differed between all combinations with either 2 steps or 200 steps. This suggests that while values between 10 and 100 do not statistically differ, the smallest and largest values do. The opposite was observed for hyenas, with many of the medium to higher values of steps being significantly different, while 2 steps was not significantly different to a higher number. However, the majority of these differences are small, and it is the category which has the most 'not significant' results. While it may not be the variable that has the strongest effect on the results, it does have a slight impact (possibly due to rare habitats or homogenous environments) and subsequently the researcher needs to select a number of steps accordingly.

This study used a variety of environmental variables, including discrete, continuous and distance to, with values measured at various points along the step (see table 4.2). Significant differences in the effect of step length could be attributed to the possibility that this value was measured at the end of the step, while distance to roads (which was often less significant) was averaged across the whole length. More research into how the environmental variables are measured has been suggested by Thurfjell et al. (2014) as an area of research that needs further exploration, and certain patterns in the results of this study certainly indicate that this warrants further research.

\section{Conclusion}

User-decisions strongly influence the results of step-selection functions and any subsequent inferences about animal movement and environmental interactions. By assuming that results would be consistent between methods, any conservation management strategies based upon SSF research could be a function of specifying unrealistic movement options. This study found that differences in individual behaviors 
have the strongest influence on the results observed. Averaging coefficients across individuals resulted in higher values when studied at an aggregate level, indicating that individual preferences are lost when studied solely at an aggregate level. Conversely, it could be stated that individual preferences exaggerate the population preference. Researchers conducting analysis on such medium sized datasets need to be aware that idiosyncratic preferences could potentially influence the results in terms of coefficient values. The method of generating available steps was important, but not as important as some have suggested (Thurfjell et al. 2014), although variations within the distributions used in this study (i.e. smaller intervals for empirical distributions) could be investigated further. Finally, while the number of steps used in comparison was the least important variable in determining coefficient values, it was still significantly different for a number of combinations. Differences between the extremes (2 and 200) suggest that a medium value may be preferable. User-decisions of SSF practitioners should subsequently be justified based on research objectives where possible and further research into other userdecisions of SSF should continue. 


\section{CHAPTER 5: INCORPORATING MOVEMENT-RELATED RESOURCE DYNAMICS INTO SPECIES DISTRIBUTION MODELLING}

\section{INTRODUCTION}

By understanding what factors define a species geographic range, more precise estimations of species resilience, range dynamics or species extinction can be made. Species distribution models (SDMs) are a widely accepted framework for studying the geographic distribution of animals, plants, and other biogeographical phenomena. SDM extrapolates species distribution data in space as well as time, applying a correlative methodology along with geospatial data of the environment (Franklin 2009; Peterson et al. 2011). These models have been used extensively for a variety of applications, including assessing the impact of climate change on species distributions (Pearson and Dawson 2003; Hijmans and Graham 2006), studying the spread of invasive species (Peterson and Vieglais 2001; Ficetola et al. 2007), and testing biogeographical hypotheses (Sagarin and Gaines 2002; Richards et al. 2007). Despite the vast potential of

SDMs to address a variety of pressing biodiversity questions, they are subject to a number of conceptual and methodological uncertainties.

The consideration of the different environmental factors that could influence a species distribution, and subsequent selection of these variables with which to train the model is an enduring issue (Guisan and Zimmermann 2000). To illustrate the individual and joint effects of the three factors deemed most important in determining species distributions, Soberón and Peterson (2005) developed the heuristic 'BAM' framework (Figure 1.1). In this framework, biotic factors (B) represent interactions with other species (i.e. competition, herbivory), abiotic factors (A) represent the physiological tolerances of a species (i.e. temperature, precipitation) and movement (M) refers to the 
area that has been or will be accessible to a species within a certain timeframe (i.e. dispersal, connectivity). $\mathbf{G}$ represents the geographical space within which the analysis occurs, $\mathbf{G}_{\mathbf{a}}$ represents the abiotically suitable area, $\mathbf{G}_{\mathbf{i}}$ is the invadable distributional area, where a species could exist if it could reach there. Finally, $\mathbf{G}_{\mathbf{0}}$ represents the occupied distributional area, and can be considered the actual distribution of a species, which is controlled by favorable abiotic, biotic and movement conditions.

While the importance of all three factors is well recognized when projecting the actual distribution $\left(\mathbf{G}_{\mathbf{0}}\right)$, seldom are all three considered together, and the majority of studies only calibrate SDMs using abiotic variables (Saupe et al. 2012). Biotic interactions are difficult to measure, particularly because species interactions can vary over time and among populations. Additionally, these interactions are characteristically superseded by abiotic factors. As such, biotic interactions are typically studied at a local scale; however, research has shown that biotic interactions have affected the distribution of species at regional, continental and global extents (Wisz et al. 2013). Similarly, movement has been under-utilized in SDM research. Many of the factors responsible for animal movement operate on an individual scale (Lagrangian approach); however in SDM, research is more often focused on population re-distribution (Eulerian approach) (Nathan et al. 2008a). Furthermore, when movement has been incorporated in SDM, its almost exclusive conceptualization has been to couple the statistical model with a measure of dispersal in response to climate change or invasive spread (Franklin 2010; Miller and Holloway 2015) or as a measure of accessibility with which to select the appropriate spatial extent for model calibration (Barve et al. 2011; Saupe et al. 2012; Qiao et al. 2015). This latter use is intended to aid interpretation of model projections and evaluation statistics such as the receiver-operating statistic. 
Movement is a pervasive feature of life, with every organism on the planet moving at some point in their life cycle. Movement processes such as dispersal and colonization are important for determining the distribution of plants and other sedentary species; however upon successful colonization, these species remain in the location that they settled. The reasons for animal movement are vast, and movement patterns range from dispersal into unknown landscapes (Chapters 2 and 3) to daily movements such as foraging and homing, These finer scale movement behaviors such as home range maintenance, foraging, and diurnal migrations have yet to be incorporated alongside SDM, despite the important role they play in the maintenance of a mobile species distribution.

Moreover, movement does not occur independently of biotic factors. The dependence of animals on food plants can be an important biotic determinant of species distributions (Kissling et al. 2008; Wisz et al. 2013). The distribution of food plants (herein biotic resources) has also long been noted as a driver of animal movement (Levin 1992), and such is the relationship between the two that the 'underlying environment' (in both terms of abiotic and biotic factors) is considered one of the four driving influences of movement in the recently introduced movement ecology paradigm (Nathan et al. 2008a). For example, many species adjust their locations and behaviors to follow resources across a range of spatial scales, meaning an individual may utilize several areas of biotic resources throughout its home range. When considered individually, these isolated areas of biotic resources may not sufficiently fulfil the requirements of an individual animal, but when considered together, they could provide suitable sustenance. Without the consideration of the neighborhood in derivation of environmental variables, any correlative model will assume that the species can survive in areas supported only by isolated patches of biotic resources, when in fact the species can only accommodate the 
existence of such conditions within its range if alternatives are available (Sinclair et al. 2010).

Various methods have been developed which attempt to address this issue by applying a measure of accessibility $(\mathbf{M})$ to the biotic factors (B). The use of a focal statistic summarizing the biotic conditions within a specified neighborhood may better represent what is available to it within its home range. For example, black grouse utilize a mixture of moorland (for mating) and woodland (for shelter) habitats, and Geary et al. (2013) identified the habitat richness of different land cover types within various neighborhood scales and incorporated this into the statistical model. The use of a focal statistic to describe the proportion of land cover types as an indicator of habitat suitability and connectivity in SDM has been found to have equal or higher predictive power than a local model, and can also help to clarify the influence of the other environmental factors that are contributing to the distribution of a mobile species (Czúcz et al. 2011; Ashcroft and Major 2013; Betts et al. 2014). The methods used to identify neighborhood features have been found to significantly alter the importance of the environmental variables in such models (Forrester et al. 2009), meaning how researchers define 'accessible' in these models can have large implications on model projections.

However, the relationship between biotic factors and movement is dynamic, as movement can greatly impact the biotic resources of an area. When organisms traverse through a landscape, biotic resources deplete while the individual is in the area (e.g. herbivory) and replenish while the individual is absent. For example, the reintroduction of wolves to Yellowstone National Park in the mid-1990s changed elk movement patterns and dramatically changed the distribution of resources in a short space of time (Turner et al. 2001). In a wolf-absent landscape, elk preferentially grazed on aspen, completely depleting entire stands (Ripple and Larson 2000). However, the reintroduction of the 113 
predator resulted in elk changing their foraging patterns, actively avoiding aspen stands where the wolves were known to be present (Fortin et al. 2005), and as a result, aspen successfully regenerated in the area. Daily consumption of resources can substantially alter the configuration of the landscape within a short period of time, and as such, it is not sufficient to consider accessibility (M) of biotic resources (B) as a static relationship. A species distribution could therefore be better explained by an ecological factor which integrates the dynamic relationship between ambulatory movements and biotic factors.

Determining the appropriate environmental factors to be used in SDM is particularly confounded by the fact that not all environmental factors operate on the same spatial or temporal scale, and that many of the drivers of species distributions interact with each other, which means that parsing out the individual influences of each variable can be difficult. While the 'BAM' framework identifies the individual and joint effects of biotic, abiotic and movement factors that influence the distribution of a species (Soberón and Peterson 2005), the three 'BAM' factors do not operate in isolation and a traditional static representation of biotic factors and accessibility may not reliably account for the complex dynamic relationship that exists between them. While the relationship between biotic factors (B) and movement (M) has been documented (Levin 1992), little (if any) research in the SDM discipline is actively being directed to address this essential issue.

\section{SPATIAL Simulation}

Spatial simulation models are simplified representations of a geographic system under study, which are used to explore, understand and better predict the behavior of the system they represent over time and in accordance with a set of predefined mathematical, statistical or other relationships (O'Sullivan and Perry 2013). Spatial simulation has been used extensively to investigate the movement and dispersal of plants and animals across a 
landscape, with a suite of approaches used including system models, cellular automata, and individual-based modelling (Tang and Bennett 2010). Due to their potential to incorporate the inherent relationship between movement and the environment, spatial simulation models are increasingly being used to understand animal movement and plant dispersal, and are beginning to be observed within the SDM framework (Martinez et al. 2012; Adams et al. 2015). For example, Martinez et al. (2012) used a parameterized individual-based model which incorporated competition, facilitation and dispersal limitations to estimate a realistic rate of tree line migration under climate change in the Pyrenees. Similarly, Adams et al. (2015) used an individual-based cellular automata model based on dispersal constraints to model invasive spread of a non-native weed in Australia over a period of five to ten years. Spatial simulation therefore has the potential to explicitly address research questions related to species distributions and movement behaviors such as dispersal under climate change, invasive spread, and home range movements.

The random walk model is a well-established methodology utilized in spatial simulation to assess simulated movement compared with a null model of movement (e.g. Miller 2015). Simple random walks constitute a movement path, whereby the next turn angle is generated randomly from all possible options. Random walk models have been extended to incorporate trends and perceived preferences; a biased correlated random walk (BCRW) describes animal movement in terms of a number of parameters, specifically directional persistence (correlation) and a consistent bias towards a given target (bias) (Codling et al. 2008). Such simulation models have successfully been implemented to explore the relationship between movement and biotic resources. For example, South (1999) simulated home range movements for the red squirrel, where individuals moved in response to a built memory map of food resources, or in response to 115 
the distribution of nest sites. At high food densities, home ranges were small, similar sized and non-overlapping, whereas the opposite was observed at low food densities, which is characteristic of red squirrel movements. Similarly, Boyer et al. (2006) developed a model that described spider monkeys foraging fruit-bearing trees based on distribution of biotic resources and memory, and found movement patterns similar to those observed by Ramos-Fernández et al. (2004). The reliability of such models to accurately simulate movement in response to the underlying dynamic biotic environment has led to BCRWs being proposed as the most flexible and discrete-step simulation model (Benhamou 2014).

Recently Duchesne et al. (2015) showed that BCRW and step-selection function (SSF) provided similar insights into the environmental factors influencing bison movement in Canada. SSF is a powerful spatial modelling approach that uses telemetry data to analyze movement patterns and resource use based on the underlying environment (Thurfjell et al. 2014). SSF considers the landscape variables associated with the movement step between two successive locations, and statistically compares these to a set of alternatively generated steps that the animal theoretically could have taken (Fortin et al. 2005). SSF has been used to evaluate the influence of the landscape features and biotic factors on a variety of species (Holloway and Miller 2014), with spatial memory recently incorporated into the statistical model (Oliveira-Santos et al. 2016). SSF subsequently models the choices actually presented to the animal as it traverses through the landscape (Chekiewicz et al. 2006), and as such could be a powerful input in spatial simulation and in particular BCRWs, although to date this has not been undertaken.

In this paper, I will explore how step-selection function can be used as a basis for spatial simulation. The use of SSF in generating bias estimates has not yet been implemented despite the vast potential to accurately and reliably inform animal116 
environment movement decisions. This model will inform the development of an environmental variable which will represent the dynamic relationship between biotic resources and movement, which can then be used within the species distribution modelling framework. This will provide researchers with an improved understanding of the relationship between biotic and movement factors, which has been absent in previous studies, and will provide an improved understanding of which factors contribute to a species distribution.

\section{METHODOLOGY}

\section{Study Species}

The oilbird (Steatornis caripensis) (Figure 5.1) is a nocturnal avian frugivore that inhabits northern South America, roosting in caves or crevices during the day, and foraging for fruit at night. In a recent study tracking oilbirds in Venezuela, Holland et al. (2009) found that the birds often roosted near their foraging sites rather than returning nightly to a cave. These findings have had important ecological consequences for the oilbird ecosystem, as the oilbirds are now seen as a primary disperser of many seeds throughout the Amazon. With an extensive home range (mean foraging distance of $44 \mathrm{~km}$ - Holland et al. 2009), an individual oilbird most likely utilizes several areas of biotic resources, making this a useful species to explore the integration of a dynamic movement-resource spatial simulation model in SDM.

The continuous monitoring of individual animals via Global Positioning Satellite (GPS) can provide more objective information on the habitat preferences of individual species than can be achieved from other sampling strategies or opportunistic sightings (Dambach and Rödder 2012). Subsequently, a number of studies have begun using the locations obtained from telemetry data as the response data in SDM (e.g. Edrén et al. 
2010; Gscwheng et al. 2012; D'Elia et al. 2015). GPS telemetry data for 40 individual oilbirds in Venezuela were obtained from Holland et al. (2009) via Movebank (2013), which resulted in a total of 5,353 locations.

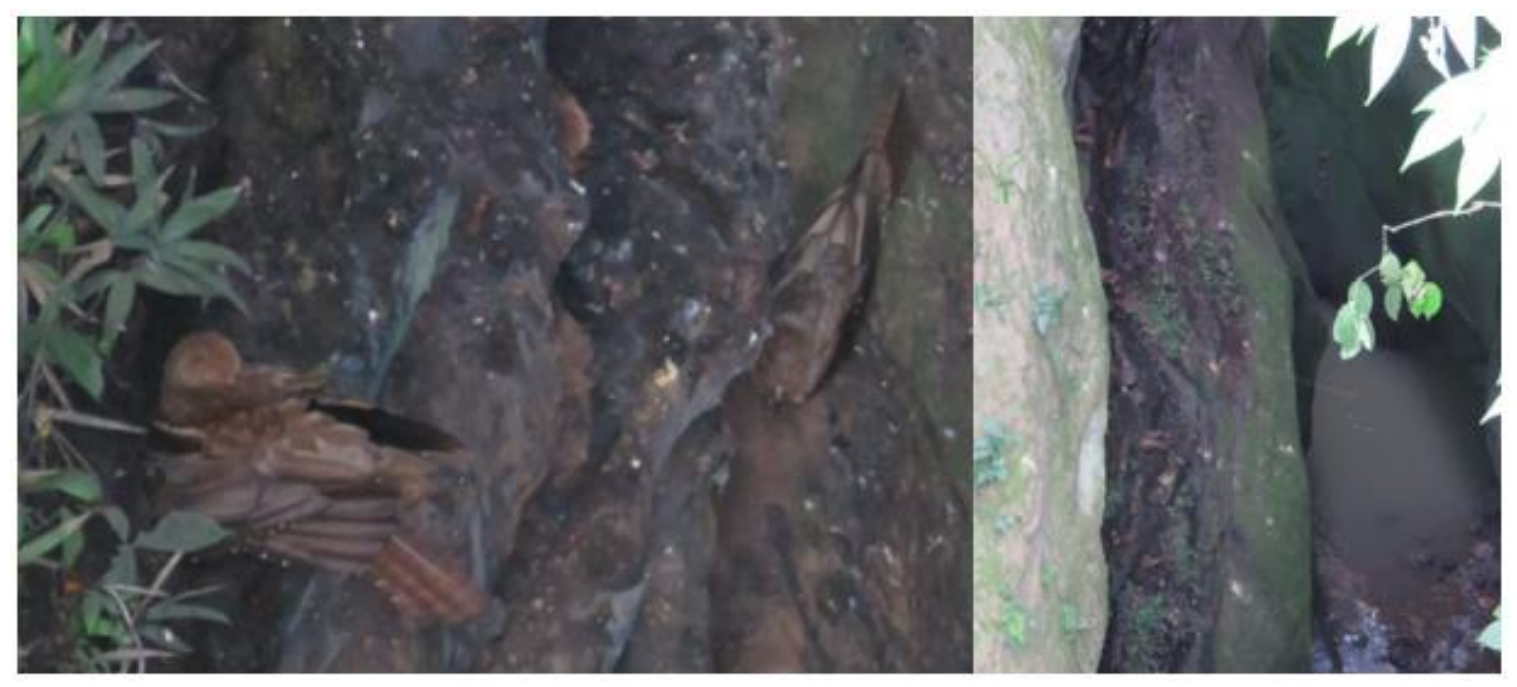

Figure 5.1: Oilbirds at Quiscarrumi Bridge, Peru, December 2014. Photo by Paul Holloway.

Despite the vast potential to use telemetry data within an SDM framework, a number of new conceptual issues related to this data structure have arisen. Spatial uncertainty is an inherent problem associated with the collection of telemetry data as observations are subject to variability in precision, measured as the distribution of differences between the central location and GPS-estimated locations (Frair et al. 2010). A further issue arising from telemetry data is the fact that the sequential observations of an individual are not independent, and subsequently spatially and temporally autocorrelated, meaning any statistical inference associated with this data requires special considerations (Boyce et al. 2010). Finally, idiosyncratic preferences of individuals may influence the habitat preferences. If a single individual contributes more locations to the 
analysis, then the subsequent SDM may be biased towards the individual's habitat preferences. To address these issues of independence, the data were filtered using the 'best daily location' approach (Abecasis et al. 2014; Trainor et al. 2014; Pikesley et al. 2014). This method selects the location with the highest accuracy for each bird within a 24 hour time period, accounting for uncertainty introduced by spatial and temporal autocorrelation and measurement error. In this study, data filtering resulted in a total of 302 points from 25 oilbirds for use as response data, with each oilbird contributing between $1-6 \%$ of the total observations. Due to the small differences in number of locations contributing to the total number of observations, and the fact that idiosyncratic preferences have not proven to significantly influence habitat suitability studies (Gscwheng et al. 2012), I chose not to further filter the data so that each individual contributed an equal amount.

\section{Species Distribution Modelling}

Maximum entropy (MaxEnt) was chosen as the statistical method to model the species-environment relationships. MaxEnt has been used extensively in SDM applications, and has been found to generate accurate predictions of species distributions using presence-only data (Elith and Graham 2009). The maximum number of iterations was set to 5000 to allow model convergence; the number of pseudo-absences was set at 10,000 (following Barbet-Massin et al. 2012), and the model incorporated only linear and quadratic features. SDMs were generated at a $10 \mathrm{~km}$ resolution (the same scale many bird atlases are collected at, e.g. Balmer et al. 2013). As Holland et al. (2009) deployed GPS tags on individuals from the Cueva del Guácharo in Northeastern Venezuela, there is unequal survey coverage of oilbirds across the entire country. If unaccounted for, this sampling bias violates the assumption of independence in the response data and could 
strongly influence the results, in particular an increased likelihood of type I errors. A bias grid was created, which can be considered the equivalent of a sampling probability surface, where the cell values reflect sampling effort and provide a weight to the pseudoabsence data used in modelling (Fourcade et al. 2014). This was produced following the method outlined by Elith et al. (2010), where a Gaussian kernel density map of the occurrence locations within $100 \mathrm{~km}$ was derived, and divided by the weighted number of terrestrial cells in the neighborhood in order to avoid the edge effects at the coastline. This was then rescaled so that values varied between 1 and 20 in order to control for the impact of extreme variation. This was then implemented in the bias file option in MaxEnt. Five different BAM scenarios were analyzed: A, B, BA, Classic BAM (cBAM), and Dynamic BAM (dBAM). The environmental variables associated with each of these scenarios are summarized in the following sub-sections as well as Table 5.1.

\section{Scenario A}

Abiotic factors (A) represent the physiological tolerances of a species (i.e. temperature, precipitation) and as such, climate variables best represent these factors. WorldClim has interpolations of observed data from $\sim 1950-2000$, with 19 bioclimatic variables derived from the monthly temperature and rainfall values in order to generate more biologically meaningful variables (Hijmans et al. 2005). Here, the 19 bioclimatic variables were considered as the abiotic factors.

\section{Scenario B}

Biotic factors (B) represent interactions with other species (i.e. competition, herbivory). The oilbird is a frugivore, suggesting that the most important biotic interactions are those with the fruit bearing trees which make up its diet. The dependence of animals on food plants can be an important biotic determinant of species distributions 
(Kissling et al. 2008; Wisz et al. 2013). Bosque et al. (1995) used seed traps to collect regurgitated seeds and identified a total of 19 species with five widespread tree species whose fruit made up a substantial part of the oilbird's diet: Euterpe precatoria, Nectandra membranacea, Ocotea floribunda, Persea caerulea and Prestoea acuminata. The distribution of these five species was predicted using observed locations from Global Biodiversity Information Facility (GBIF 2015a; 2015b; 2015c; 2015d; 2015e), the 19 bioclimatic variables (Hijmans et al. 2005) and projected using MaxEnt with default settings (with the exception of 5000 minimum iterations to allow model convergence Phillips et al. 2006). The threshold of presence was set as the maximum sensitivity plus specificity, and distribution maps were generated at a $1 \mathrm{~km}$ resolution. A categorical variable representing presence or absence of the five species within the $10 \mathrm{~km}$ observation were used as the biotic factors.

\section{Scenario $B A$}

This scenario projects the invadable distribution $\left(\mathbf{G}_{\mathbf{i}}\right)$ in the BAM framework. This model uses the intersection of suitable abiotic and biotic factors to determine distribution. The 19 bioclimatic variables and the five presence absence maps of the food species were used as the input variables.

\section{Scenario Classic BAM}

Movement factors $(\mathbf{M})$ refer to the area that has been or will be accessible to a species within a certain timeframe. Accessibility to biotic resources was identified by using a distance buffer. In their study on the foraging patterns of the oilbird, Holland et al. (2009) found that the mean foraging distance of the oilbird was $44.4 \mathrm{~km}$. The presence or absence of the five fruit-bearing tree species was identified within a focal area of $50 \mathrm{~km}$ for each $10 \mathrm{~km}$ observation. This was represented by a categorical binary representation 
of presence / absence within a focal area of 50km (n.b. no resampling is undertaken and the resolution remains at $10 \mathrm{~km})$.

\section{Scenario Dynamic BAM}

A variable which summarizes the dynamic relationship between movement and biotic resources within the $50 \mathrm{~km}$ focal area was created using spatial simulation. Movement was simulated as a BCRW parameterized on the SSF model, with factors including land cover, presence of resources, and memory. Identifying the survival of individual agents in spatial simulation by the amount of energy consumed is common in individual-based models (e.g. Wilensky and Reisman 1999; Ahearn et al. 2001; Silby et al. 2013), and captures the dynamic relationship between movement and biotic resources. Individuals move in response to the spatial configuration of biotic resources, consuming resources and energy as they move. The biotic resources will subsequently respond by depleting if consumed by individuals. If an individual does not obtain enough energy, it will die. The spatial simulation was run for one year period with 1,000 oilbirds (the $90^{\text {th }}$ percentile of oilbird population size in Venezuela - Herrera 2003). The survival rate will be the input into the SDM. A high value indicates a landscape with a large amount of accessible and sustainable biotic resources, while a low value will indicate insufficient resources or inaccessible resources. In following with the current standard for modelling, a thorough account of the design of the model is outlined using the ODD protocol (Overview, Design Concepts and Details - Grimm et al. 2006; 2010) in Appendix 5, and the model is outlined in Appendix 6. This layer will be incorporated along with the abiotic factors. 
Table 5.1: BAM Scenarios used to explore oilbird distributional patterns.

\begin{tabular}{|c|c|}
\hline BAM Scenario & Explanation \\
\hline $\mathbf{A}$ & $\begin{array}{l}\text { Abiotic factors (A) represent the physiological tolerances of a species (i.e. temperature, } \\
\text { precipitation). Here, the } 19 \text { bioclimatic variables (Hijmans et al. 2005) were considered as } \\
\text { the abiotic factors. }\end{array}$ \\
\hline $\mathbf{B}$ & $\begin{array}{l}\text { Biotic factors (B) represent interactions with other species (i.e. competition, food } \\
\text { sources). A categorical variable representing presence or absence of the five species } \\
\text { within the } 10 \mathrm{~km} \text { observation were used as the biotic factors. }\end{array}$ \\
\hline $\mathbf{B A}$ & $\begin{array}{l}\text { This scenario projects the invadable distribution }\left(\mathbf{G}_{\mathbf{i}}\right) \text { in the classic BAM framework. The } \\
19 \text { bioclimatic variables and the five presence absence maps of the food species were used } \\
\text { as the input variables. }\end{array}$ \\
\hline Classic BAM (cBAM) & $\begin{array}{l}\text { Movement factors }(\mathbf{M}) \text { refer to the area that has been or will be accessible to a species } \\
\text { within a certain timeframe. The presence or absence of the five fruit-bearing tree species } \\
\text { will be identified within a focal area of } 50 \mathrm{~km} \text { for each } 10 \mathrm{~km} \text { observation. This will be } \\
\text { represented by a categorical binary representation of presence / absence within a focal } \\
\text { area of } 50 \mathrm{~km} \text {, and the } 19 \text { bioclimatic variables. }\end{array}$ \\
\hline Dynamic BAM (dBAM) & $\begin{array}{l}\text { Spatial simulation will be used to create a variable which summarizes the dynamic } \\
\text { relationship between movement and biotic factors within the } 50 \mathrm{~km} \text { focal area. The } \\
\text { survival rate of } 1000 \text { oilbirds will be the input layer representing BM, and the } 19 \\
\text { bioclimatic variables representing A. }\end{array}$ \\
\hline
\end{tabular}




\section{Model Evaluation}

Where possible, evaluation should be undertaken with an independent test dataset. There are 32 unique oilbird presence observations in Venezuela on the Global Biodiversity Information Facility (GBIF 2016), and these were used as the test dataset. Multiple evaluation metrics should be used when reporting the evaluation of SDM projections due to the potential differences reported between metrics (Liu et al. 2005; Franklin 2009). As the test dataset consists of presence-only data, the accuracy metrics discussed in Chapters 2 and 3 cannot be applied as there is no test absence data. Subsequently, three presence-only metrics were calculated for each projected distribution of oilbirds.

The lowest possible threshold (LPT) is the threshold (the probability value above which a case is predicted to be positive) that would result in zero omission errors. The minimum predicted area (MPA) is the threshold value that minimizes omission errors, but at the same time minimizes the area of the map predicted to be suitable (Franklin 2009). The MPA represents the threshold value that encompasses a predefined proportion of observed species occurrences (in this case 90\%). The Boyce Index is based on a moving window analysis across the range of predicted values, and uses Spearman rank correlation coefficient to measure the monotonic increase in the predicted-to-expected frequency ratio with increasing habitat suitability (Boyce et al. 2002). In an extensive comparison among presence-only accuracy metrics, Hirzel et al. (2006) concluded that the Boyce Index calculated with a continuous moving window was the most robust and informative metric. This metric was implemented using the ecospat package (Broenniman et al. 2014), and ranges from -1 to 1 , with 0 indicating predictions indifferent from a random model. 


\section{RESULTS}

\section{Step-Selection Function}

The final step-selection function model which was used in the spatial simulation identified several significant variables that identify the landscape, resource and memory factors associated with oilbird movement (Table 5.2). Oilbirds preferentially chose movement paths into landscapes which they have previously visited and knew to have resources, and also favorably selected steps into unknown habitat rather than returning to a location which they had visited, but knew not to have resources. Oilbirds also favored movement steps into evergreen broadleaf forests and croplands over movement steps into other land cover classes, while selecting steps away from rivers.

Table 5.2: The results of the conditional logistic regression model used for stepselection function scores. * significant at $\alpha<0.1, * *$ significant at $\alpha<0.05$.

\begin{tabular}{l|c} 
Variables & Coefficient (Standard Error) \\
\hline Evergreen Forest & $0.90630(0.46580)^{*}$ \\
Cropland & $0.79150(0.54720)$ \\
Rivers & $-0.00020(0.00004)^{* *}$ \\
Memory & $-3.11500(0.97270)^{* *}$ \\
Resources & $-1.34000(0.50440)^{* *}$ \\
Memory*Resources & $3.39300(0.98660)^{* *}$
\end{tabular}

Figure 5.2 illustrates the movement trajectories of an oilbird over a two month period for one $50 \mathrm{~km}$ grid cell (starting from the center of the grid). The difference between a random walk (Figure 5.2a), a correlated random walk parameterized on empirical turn angles and steps lengths (Figure 5.2b), and a BCRW parameterized on SSF (Figure 5.2c) is evident. The random walk trajectories do not take into account the underlying environment or memory, meaning that the random walk model is characterized by more frequent and longer movement steps and as such the overall 
trajectory appears unrealistic of actual oilbird movement, with the individual appearing to cover the entirety of the grid. While the correlated random walk is restrained compared to the random walk, the area of the $50 \mathrm{~km}$ it covers is still magnitudes larger. The BCRW path consists of several smaller movement steps, followed by a longer step, with a high number of steps returning to previous locations where the interaction between memory and resources is positively influencing the step. The difference in the spatial configuration of the underlying landscape also influences the movement paths. Where the region is made up of mainly evergreen forest (Figure 5.2d), the SSF movement trajectories are widely dispersed across the region and the non-landscape variables exert more influence, whereas the BCRW path does not extend into the south-west of the grid due to the presence of other habitat and cropland which are less favorable steps than evergreen forest (Table 5.2).
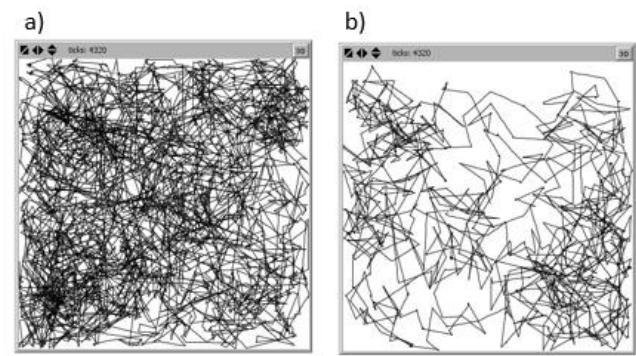

c)

d)
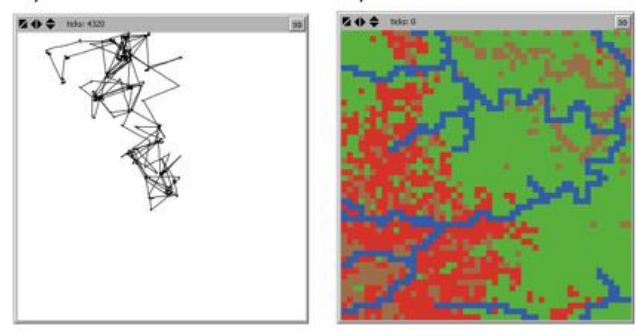

Figure 5.2: Movement trajectories for movement steps of an oilbird from a) random walk model, b) correlated random walk (parameterized on empirical turn angle and step length), c) biased correlation random walk (parameterized on step-selection function. d) the landscape of the grid 
Table 5.3: The three accuracy metrics of the five BAM scenarios: Lowest possible threshold (LPT), minimum predicted area (MPA), and the Boyce index (BI).

\begin{tabular}{l|rrr}
\multicolumn{1}{c}{ LPT } & MPA & BI \\
\hline $\mathbf{A}$ & 0.0002 & 0.0197 & 0.8210 \\
$\mathbf{B}$ & $\mathbf{0 . 0 1 7 2}$ & $\mathbf{0 . 0 2 9 6}$ & 0.0580 \\
$\mathbf{B A}$ & 0.0001 & 0.0256 & 0.7600 \\
cBAM & 0.0004 & 0.0220 & 0.6350 \\
dBAM & 0.0002 & 0.0271 & $\mathbf{0 . 8 9 2 0}$
\end{tabular}

Figure 5.3 shows the spatial distribution based on the different models, using a threshold of 0.0302 (which represents the proportion of presence points used to generate the model compared to the number of pseudo-absences). The distributions differ quite substantially between scenarios, with an increase in the number of BAM factors incorporated in the SDM reducing the area predicted as present. The dBAM scenario projected $211,100 \mathrm{~km}^{2}$ as present compared to the A or B scenarios which projected $351,900 \mathrm{~km}^{2}$ and $394,100 \mathrm{~km}^{2}$ as present respectively. In particular, the dBAM scenario removes the distribution that was predicted by $\mathrm{B}$ and $\mathrm{A}$ scenarios in the center of the country, and projects a fragmented distribution around the boundary of the country. Conversely, the cBAM scenario predicts a relatively continuous surface of oilbird presence around the whole country, and projects the most area as present, a likely explanation for the higher accuracy of LPT and MPA (table 5.2). However if a measure of specificity could be incorporated into the accuracy metrics, the B scenario would likely be penalized for over-prediction.

Figure 5.4 shows the variation in the three data layers which represent biotic factors and movement. The differences in the presence of the five tree species at a $1 \mathrm{~km}$ resolution (Figure 5.4a) compared to when presence or absence is viewed at a $50 \mathrm{~km}$ neighborhood scale at a $10 \mathrm{~km}$ resolution (Figure $5.4 \mathrm{~b}$ ) is evident. It can be seen by 
representing presence/absence at a $50 \mathrm{~km}$ focal area, presence is overestimated across Venezuela, particularly in the south of the country. Finally Figure 5.4c represents the new dynamic movement and biotic resource layer, and identifies the number of oilbirds remaining after a one-year period. It can be seen that many locations of biotic resources are unsustainable, with large areas of the projected tree species distributions from Figures 5.4a-b disappearing. This layer better represents the dynamic relationship between biotic resources and movement, and does well to identify core areas of oilbird suitability, sustainability and accessibility.

\section{DISCUSSION}

The aim of this chapter was to investigate how spatial simulation could be used to represent the relationship between movement and biotic factors. The result of this simulation is a variable which represents the dynamic relationship between biotic resources and movement, and can be incorporated in the statistical model to better explain the factors impacting the distribution of oilbirds in Venezuela. The inclusion of a variable which accounts for the dynamic relationship between movement and biotic resources improved the accuracy (when measured as the Boyce Index) of the SDM (Table 5.2). The Boyce Index is considered the most robust presence-only accuracy metric due to its ability to measure the monotonic increase in the predicted-to-expected frequency ratio with increasing habitat suitability, with a higher value representative of a more valid model (Hirzel et al. 2006; Franklin 2009). The Boyce Index was highest for the dBAM model, suggesting that incorporating a variable which measured the relationship between $\mathbf{M}$ and $\mathbf{B}$ was advantageous to the SDMs projection capacity. This argument is furthered by the fact that this layer had the highest relative contribution of all 
the 'BAM' variables used the final model $(37.6 \%)$, or in other words the most influence on the projected environmental and geographic distribution of oilbirds.
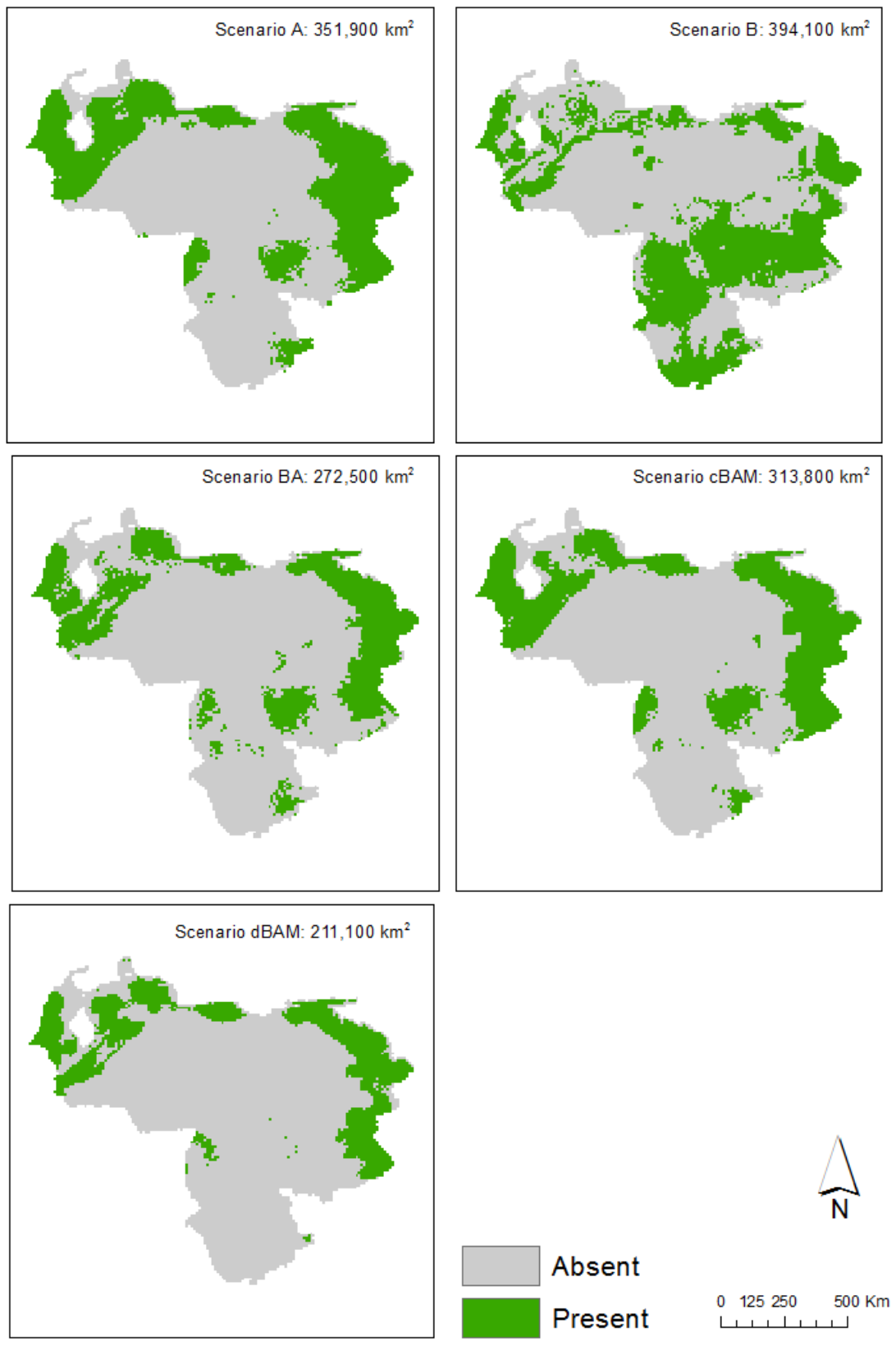

Figure 5.3: Spatial distribution of oilbirds in Venezuela from the five BAM scenarios. Threshold chosen as the proportion of presences compared to pseudoabsences. 


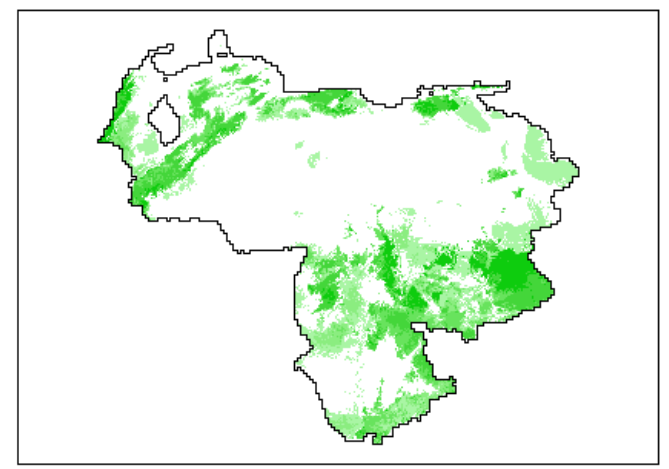

Number of Plant Species Present (1 $\mathrm{km}$ resolution)

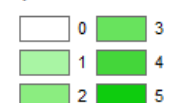

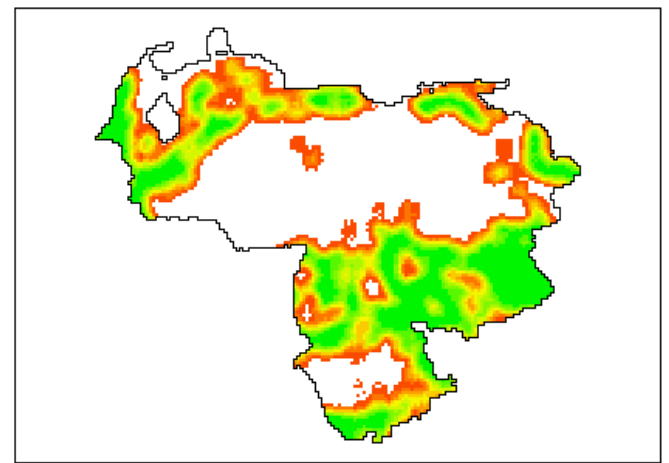

Number of Oilbirds that can be Sustained within a $50 \mathrm{~km}$ focal area (10 $\mathrm{km}$ resolution)

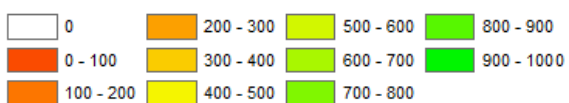

Figure 5.4: The data layers which represent biotic resources and movement factors - a) the number of plant species present at a $1 \mathrm{~km}$ resolution, b) The number of plant species present within a $50 \mathrm{~km}$ focal area at a $10 \mathrm{~km}$ resolution, and c) the number of oilbirds that can be sustained within a $50 \mathrm{~km}$ focal area (the dynamic $\mathbf{B}$ and $\mathbf{M}$ layer).

The availability of food resources depicted here (presence/absence of the five vegetation species) could be considered a simplistic representation of biotic factors; however this is how $\mathbf{B}$ and $\mathbf{M}$ have been combined in SDM to date. While the model trained only on $\mathbf{B}$ recorded the highest LPT, the variation in habitat suitability scores is 
low, particularly because only five categorical variables were included in the analysis. This is supported by the low Boyce Index for B (0.06) which indicates that the model is no different from a random model. One criticism of using a static variable of biotic factors is that if any of the alternative biotic resources 'disappear' (for example through climate or land cover change), then the configuration of the landscape may be such that the patches of available resources are no longer accessible, resulting in a very sudden constriction of the species geographic distribution (Sinclair et al. 2010). While a static variable of accessibility (M) to biotic resources (B) addresses this, the results from this study indicate that it does not do well in correctly predicting the distribution, in part because these measures of $\mathbf{B}$ and $\mathbf{M}$ treat the entire focal area as homogenous, when in fact it can be highly heterogeneous in terms of resources and land cover. The use of a dynamic environmental layer of biotic resources and movement removes the assumption of homogeneity across the distribution of food species, and delineates between accessible and sustainable areas of biotic resources (e.g. source populations) from isolated and unsustainable areas of biotic resources (e.g. sink populations). This results in a better indicator of the relationship between biotic resources and movement, and removes some of the uncertainty introduced by source and sink populations for mobile species.

While biotic factors have the potential to strongly influence the distribution of a species through processes such as competition, herbivory, and facilitation, they are also a function of abiotic factors. For example, a competing species may only be able to tolerate a portion of the abiotic conditions across the study species entire range, and will only be able to interact where these abiotic conditions are favorable. As the distribution of the five plant species was based on the 19 bioclimatic variables, it could be argued that the B scenario was actually representative of BA. However, by its definition in the BAM framework, A represents the physiological tolerances of the species which is being 
studied, meaning that the abiotic conditions that define the food species may not be the same as the physiological tolerances of the oilbird.

Nevertheless, this is still an important aspect of the BAM framework which should be acknowledged. None of the three factors is completely independent of another. While this study focused on the dynamic relationship between movement and biotic factors, interactions between and among all three BAM factors occur, and it may be theoretically difficult to parse out the influence of each individual factor. For example, abiotic factors have been found to influence the movement of animals, with Delattre et al. (2013) finding that the dispersal distances of meadow brown butterflies were greater across fragmented landscapes at cooler temperatures, but greater across continuous landscapes at warmer temperatures. In extreme instances (such as that observed at Yellowstone National Park), the interaction between elk movement and their selective herbivory on aspen and willow resulted in changes in the river channels and subsequently the surrounding microclimates (Beschta and Ripple 2006). Therefore, future BAM research should not only focus on the individual and combined effects of the three factors, but also identify a clear methodology with which to successfully model the undoubted dynamic relationships between all three factors.

The incorporation of SSF within a spatial simulation model is novel, and the results suggest that this is a method that performs well. The movement trajectories of the individual oilbirds were characteristic of several short steps followed by a long one (Figure 5.2). This is distinctive of many home range movements, in particular foraging theory, and has been the basis for other simulations such as the Lévy walk (Boyer et al. 2006; Benhamou 2007; Plank and Edwards 2011). The benefit of using SSF within a BCRW over other implementations is that it includes the results of conditional logistic regression. As movement is simulated using a statistical model generated from observed 
movements, more realistic movement behaviors are simulated compared to a model specified solely on subjective assumptions, and results better illustrate the influences of the environment on actual movements (Duchesne et al. 2015). Similarly, SSF can be deconstructed to capture the variations in movement patterns across days and seasons (e.g. Bjorneraas et al. 2011). One potential issue is the dependency of SSF on telemetry data. However, BCRW could still be utilized to explore the dynamic relationship between biotic resources and movement, and would still be preferential to a static measure of $\mathbf{B}$ and M. SSF is therefore a powerful input in spatial simulation, and the results from this chapter demonstrate this.

A recent criticism of SSF has been the lack of methodology with which to incorporate a measure of memory (Fagan et al. 2013; Duchesne et al. 2015). To ignore the effects of memory when analyzing movement decisions made by animals can lead to biased estimates of resource selection coefficients (Oliveira-Santos et al. 2016). The results in this study agree with Oliveira-Santos et al. (2016); memory is an important factor to consider within an SSF (Table 5.2). Furthermore the importance of considering memory of biotic resources rather than simply spatial memory irrespective of the landscape should be considered. Memory does not operate independently of information or events, and when memory was the only factor considered, oilbirds avoided moving into habitats that they had previously visited, but when modelled as an interaction with awareness of biotic resources, oilbirds conversely selected locations they had previously visited and knew had resources. Memory can be modelled in a number of ways, and the assumption of memory in this chapter relied on the theory that time spent in an area contributed to the spatial memory of that individual. While this description of memory is a simplified approximation, it does illustrate that this is an important process which should be accounted for when simulating movement, and as such should help foster 133 
future SSF studies which look to incorporate spatial memory. Future SSF research should identify how wildlife behavior studies have incorporated memory, and whether these considerations of movement could be used to inform modelling approaches.

\section{CONCLUSION}

The 'BAM' framework identifies the individual and joint effects of biotic, abiotic and movement factors that influence the distribution of a species (Soberón and Peterson 2005); however the three 'BAM' factors do not operate in isolation and a traditional static representation of biotic factors and accessibility may not reliably account for the complex dynamic relationship that exists between them. Spatial simulation has been used extensively to investigate the movement and dispersal of plants and animals across a landscape, and was subsequently used to generate an environmental layer which represented the dynamic relationship between biotic resources $(\mathbf{B})$ and movement $(\mathbf{M})$. The use of this layer improved the accuracy and ecological realism of SDM projections compared to other widely used BAM scenarios. The use of step-selection function as an input into the spatial simulation was novel. SSF of oilbird movement was estimated using landscape features, biotic resources, and memory, and the results demonstrated realistic movement patterns. Furthermore, the use of an interaction term between memory and biotic resources improved the final SSF model, and aided in the interpretation of how oilbirds were selecting movement steps. Finally, the use of a Lagrangian approach within SDM was also novel. Integrating this type of movement process into an SDM framework was successful, and future research should continue to explore how a Lagrangian approach can be used to better understand the relationship between abiotic, biotic and movement factors. 


\section{CHAPTER 6: MOVEMENT ACROSS SCALES: INCORPORATING BOTH DISPERSAL AND REGULAR MOVEMENTS IN SPECIES DISTRIBUTION MODELS.}

\section{INTRODUCTION}

Dispersal is an important process in determining the redistribution of organisms, contributing to colonization of new areas, range shifts, and the mixing of gene pools between and within populations across a range of time scales from decades to millennia (Jønsson et al. 2016). It is becoming more common in SDM applications to couple some measure of dispersal with future projections of suitable abiotic and biotic habitat in order to distinguish between areas that are potentially (abiotically and biotically) suitable and accessible with areas which are potentially suitable but inaccessible (Franklin 2010; Bateman et al. 2013; Miller and Holloway 2015). A number of methods have been proposed to simulate dispersal for these applications, ranging from simple assumptions of either 'all' or 'nothing' (Araujo et al. 2006; Luo et al, 2015; Riordan et al. 2015), to complex probabilistic dispersal kernels (Summers et al. 2012; Alagador et al. 2014; Bush et al. 2014). As the research described in this dissertation illustrates, the choice of dispersal model used can significantly alter the accuracy and uncertainty in future projections (Chapters 2 and 3), and future research should continue to investigate how this Eulerian (population or top-down) approach influences the accuracy and uncertainty of future projections which require a simulation of dispersal in response to global change.

Regular movements are also an important determinant of a species distribution, with movement behaviors such as foraging (searching for food), homing (returning to home), bounding (marking home range limits), and biotic interactions (e.g., herbivory) all contributing to the maintenance of a mobile species distribution. Mobile species utilize several patches of biotic resources (defined here as food sources) throughout their home 
range. When considered individually, these isolated areas of biotic resources may not sufficiently fulfill the requirements of an individual animal, but when considered at a neighborhood scale, they provide suitable sustenance to that individual. Model calibration in SDM therefore needs to account for the dynamic spatial variation of biotic resources within the distribution of mobile animals. The use of spatial simulation to represent the dynamic relationship between movement and biotic factors improved both the accuracy of SDMs compared to other BAM scenarios and substantially improved the ecological realism of projections (Chapter 5). Future research should subsequently continue to investigate how this Lagrangian (individual or bottom-up) approach can be used to better understand the relationship between abiotic, biotic, and movement factors.

In spite of research that has demonstrated how each of these types of movement is important in SDM projections, incorporating movement across multiple scales has not been addressed in SDM research (Chapter 1). Components at fine scales can interact and accumulate across space and time, producing patterns and processes at broader scales (Peters et al. 2007). Therefore, the emergent properties of regular movements (such as foraging and habitat connectivity) can effectively alter the dispersal outcomes of populations which are responding to changes in climate (Heffernan et al. 2014). For mobile species that are dependent on a number of different patches of biotic resources, the loss of only a small amount of these could result in a sudden and dramatic range constriction (Sinclair et al. 2010). For example, Figure 6.1 is an observation of a hypothetical species, along with the spatial distribution of biotic resources within this area. For an individual to be present, all six patches of biotic resources need to be used, which requires these locations to be connected and sustainable (Figure 6.1). When projecting into the future with the assumption that there are no changes in abiotic conditions, there are four possible scenarios that may be observed. The configuration of 
biotic resources could remain the same (scenario a), or the area of biotic resources increases (scenario b). Both of these scenarios would result in the continued presence of the species. Alternatively, the configuration of this area could be altered, such that all the areas are no longer connected and this fragmentation results in the inaccessibility of biotic resources across the entire observation (scenario c) or the area of resources declines, such that the amount of biotic resources no longer sustains the individual (scenario d).

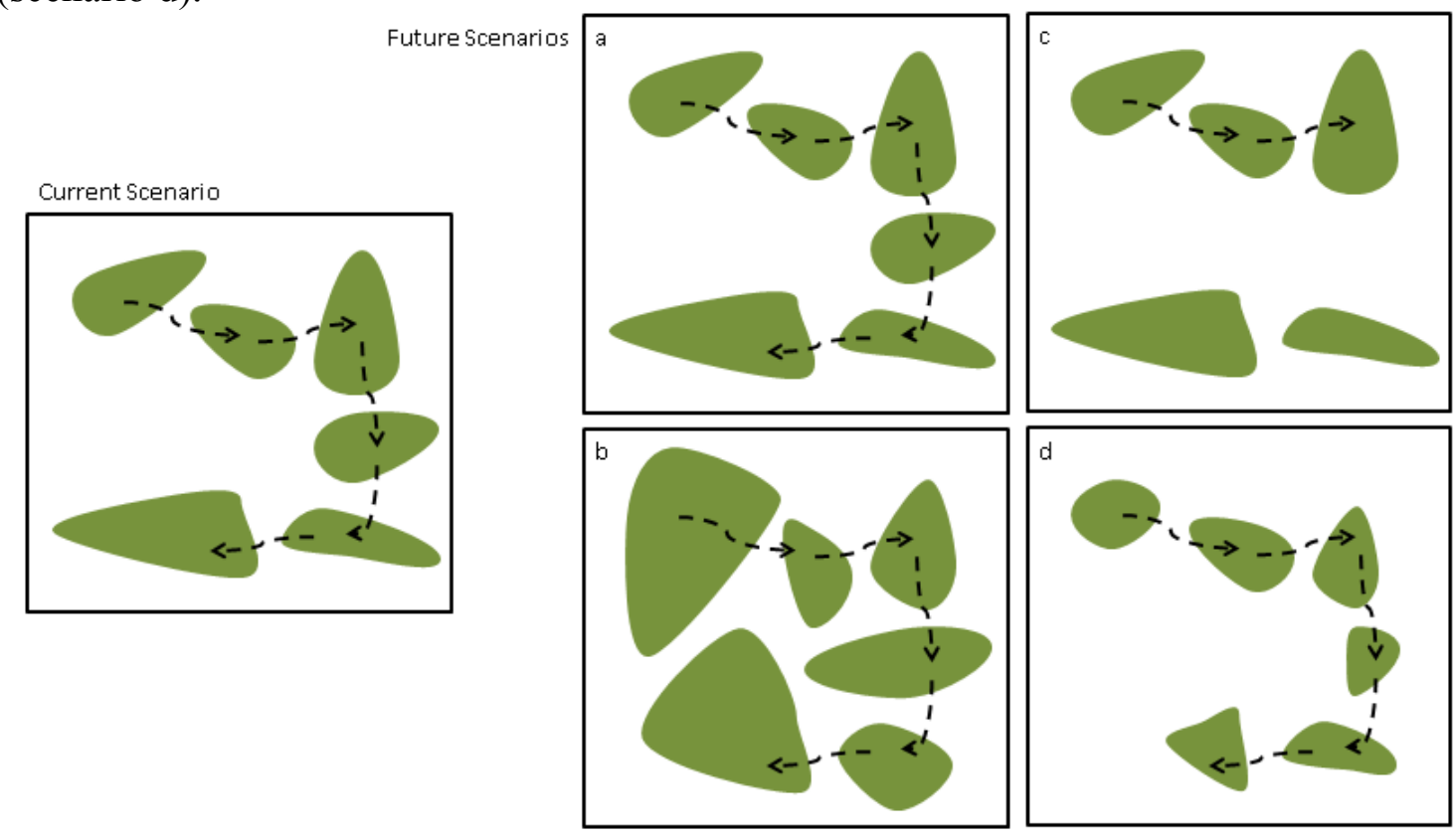

Figure 6.1: Conceptual diagram of the four possible scenarios associated with biotic resources in future time.

A static representation of biotic factors and movement (which is the predominant method of assessing accessibility to biotic resources in SDM studies that have addressed this) would project this mobile species as being present under all future scenarios, failing to acknowledge the reduced connectivity and amount of food in scenarios $\mathrm{c}$ and $\mathrm{d}$. This issue is particularly relevant for studies projecting into future space and time, since the 
influences of abiotic and biotic factors operate on different spatiotemporal scales, but both factors contribute to the future distribution. The future distribution of species under climate change and land cover changes are therefore highly dependent on both the processes of fine-scale regular movements and broad-scale dispersal into new habitats.

The focus of this final chapter is to illustrate how both broad-scale dispersal and fine-scale regular movements can be incorporated in the SDM framework. Speciesenvironmental relationships for oilbirds across South America will be projected for 2001 based on three BAM scenarios: A, cBAM and ABAM. These relationships will then be extrapolated to 2010 with the future projections of oilbird distributions coupled with four dispersal models in order to identify both (BAM) suitable and (dispersal) accessible future habitat.

\section{METHODOLOGY}

The oilbird (Steatornis caripensis) will again be used in this case study. The dynamic relationship between regular movements and biotic factors was established as an important determinant in the maintenance of the oilbirds distribution, with the spatial simulation model outlined in detail in Chapter 5 and Appendices 5 and 6. Presence data for use in the statistical model were obtained from the Global Biodiversity Information Facility (GBIF 2016) with observations from South America pre-2001 used for model calibration, and observations from post-2010 used as independent test data for model evaluation.

Abiotic factors (A) represent the physiological tolerances of a species (i.e. temperature, precipitation) with climate variables best representing these factors. Climate Research Unit (CRU) time-series 3.10.01 data of monthly average precipitation, and minimum and maximum temperature was obtained (University of East Anglia CRU, 
Jones and Harris 2013) at a $50 \mathrm{~km}$ resolution. Climate data was then delineated into December, January and February, and June, July and August averages for the 5 years preceding 2001 and 2010 .

Biotic factors (B) represent interactions with other species (i.e. competition, herbivory). Again, the distributions of the five main food species of the oilbird (Bosque et al. 1995) were used to identify favorable biotic factors. The distribution of these species was predicted using observed locations from Global Biodiversity Information Facility (GBIF 2015a; 2015b; 2015c; 2015d; 2015e), 1km monthly average precipitation, and minimum and maximum temperature data for the 5 preceding years, calculated by identifying the variation between the 5 year average at $50 \mathrm{~km}$ resolution (University of East Anglia Climatic Research Unit 2008) and the 50 year average at $1 \mathrm{~km}$ resolution (Hijmans et al. 2005), and projected using MaxEnt with default settings (with the exception of 5000 minimum iterations to allow model convergence - Phillips et al. 2006). The threshold of presence was set as the maximum sensitivity plus specificity, and distribution maps were generated at a $1 \mathrm{~km}$ resolution. The spatial resolution of the oilbird SDM $(50 \mathrm{~km})$ is equivalent to the mean oilbird foraging distance identified by Holland et al. (2009). Therefore, this implementation of $\mathbf{B}$ in BAM is closer to the cBAM scenario outlined in Chapter 5 whereby the accessibility of biotic resources is estimated as a static variable for a $50 \mathrm{~km}$ grid. The presence or absence of the five tree species along with the climate variables will be used in this cBAM scenario.

Finally, scenario dBAM will be calculated using the climate variables, and the dynamic movement-related resource model based on the BCRW parameterized on SSF (outlined in Chapter 5). For each 50km observation, the sustainability of 1000 oilbirds will be used as the layer representing the dynamic relationship between $\mathbf{B}$ and $\mathbf{M}$. Due to the coarse resolution of the oilbird distribution, a local value that summarizes the 139 
dynamic relationship between movement and biotic resources will be calculated for each $50 \mathrm{~km}$ grid and no focal statistic will be calculated. With the mean foraging distance of the oilbird less than $50 \mathrm{~km}$, there is little ecological reason to investigate sustainability and accessibility at a wider neighborhood scale. Also, this decision was necessary due to the inherent tradeoff in computer resources needed to conduct this research at a coarser extent (continental v regional) and resolution $(50 \mathrm{~km} \mathrm{v} 10 \mathrm{~km})$.

No single dispersal model has been identified as consistently outperforming all others, and therefore, four dispersal models were coupled with the statistical model of future projections. Unlimited dispersal (UD) and no dispersal (XD) are considered important measures of capturing the uncertainty in future projections, to which other dispersal models inferring actual dispersal abilities can be compared. No natal dispersal distances for the oilbird are documented; however, Sutherland et al. (2000) compiled a database of global terrestrial birds and mammals, and identified that natal dispersal distance of birds was strongly correlated to body mass and diet. Following their equation, and the average body mass recorded by Holland et al. (2009) of $0.4197 \mathrm{~kg}$, the median and maximum natal dispersal distances of the oilbird were estimated as $2.18 \mathrm{~km}$ and $36.46 \mathrm{~km}$ respectively. The spatial resolution of the SDM is $50 \mathrm{~km}$ and therefore coarser than the maximum natal dispersal distance estimated for oilbirds. With the issues identified in Chapter 3 regarding the resolution of a coarse SDM over-estimating dispersal potential, and the variability in fitting theoretical dispersal kernels to species identified in Chapter 2, a fixed rate (FR) method was chosen as the dispersal model. While the use of a mean natal dispersal distance often under-predicted the future distribution in an FR dispersal model (Chapter 2 and 3), by selecting both the median and maximum natal dispersal distances, this should identify the differences between the two and address some of the uncertainty. The latter model with maximum dispersal distance 
is closer to the discrete dispersal kernel implementation without the uncertainty of fitting a theoretical kernel. The use of a maximum dispersal distance was found to consistently obtain high accuracy values and successfully incorporate long-distance dispersal (Chapters 2 and 3). For a time period (T) of 9 years, and a breeding age (A) of 1, the median natal dispersal distance of an oilbird is $19.62 \mathrm{~km}$ or 1 grid cell, and the maximum natal dispersal distance of an oilbird is $328.14 \mathrm{~km}$ or 7 grid cells (at $50 \mathrm{~km}$ resolution). Due to the computational resources required to undertake the spatial simulation and the relatively short time period of study, this model will be run as a 1 time-step model. This will further reduce the uncertainty associated with projecting suitability and dispersal across a coarse resolution.

\section{RESUlts}

Differences among BAM scenarios were observed, with scenario cBAM projecting the most area as present as well as the most accurate predictions (Figures 6.2, 6.3). The incorporation of a static factor representing biotic factors and movement increased the spatial extent predicted present compared to scenarios A and dBAM. However, the area predicted present by scenario A was less than scenario dBAM, which contradicts the findings from the previous chapter. Despite this, the incorporation of the dBAM layer identifying oilbird sustainability reduces the area predicted as present compared to cBAM, which further supports the argument that not identifying connectivity and accessibility could potentially over-predict the distribution. When projections are visually compared (Figure 6.2), the areal extent does not appear to vary, although a difference of $1,030,000 \mathrm{~km}^{2}$ was recorded between cBAM UD and A XD (Figure 6.3). 


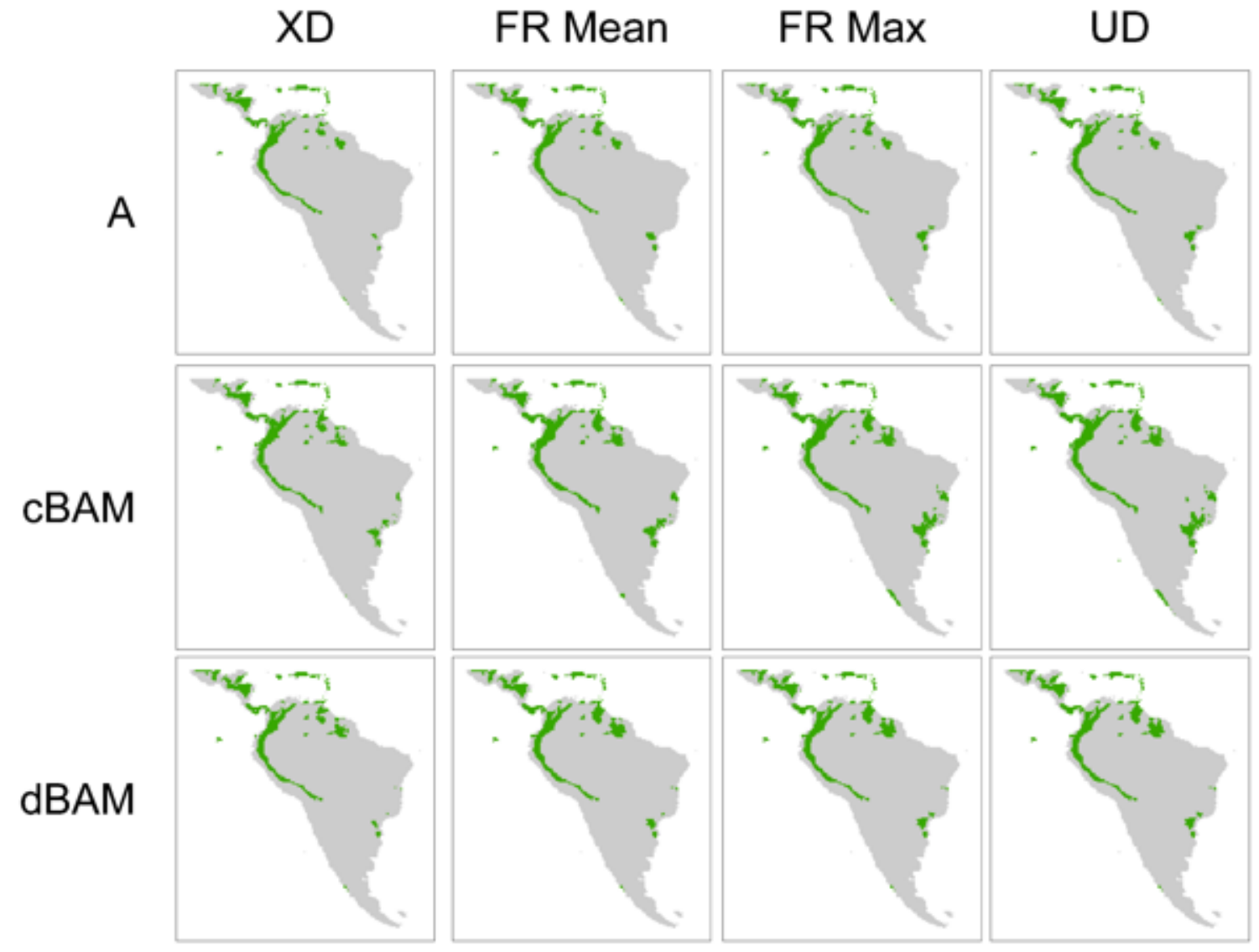

Figure 6.2: Areal extents predicted as present by varying dispersal models and BAM scenarios. 

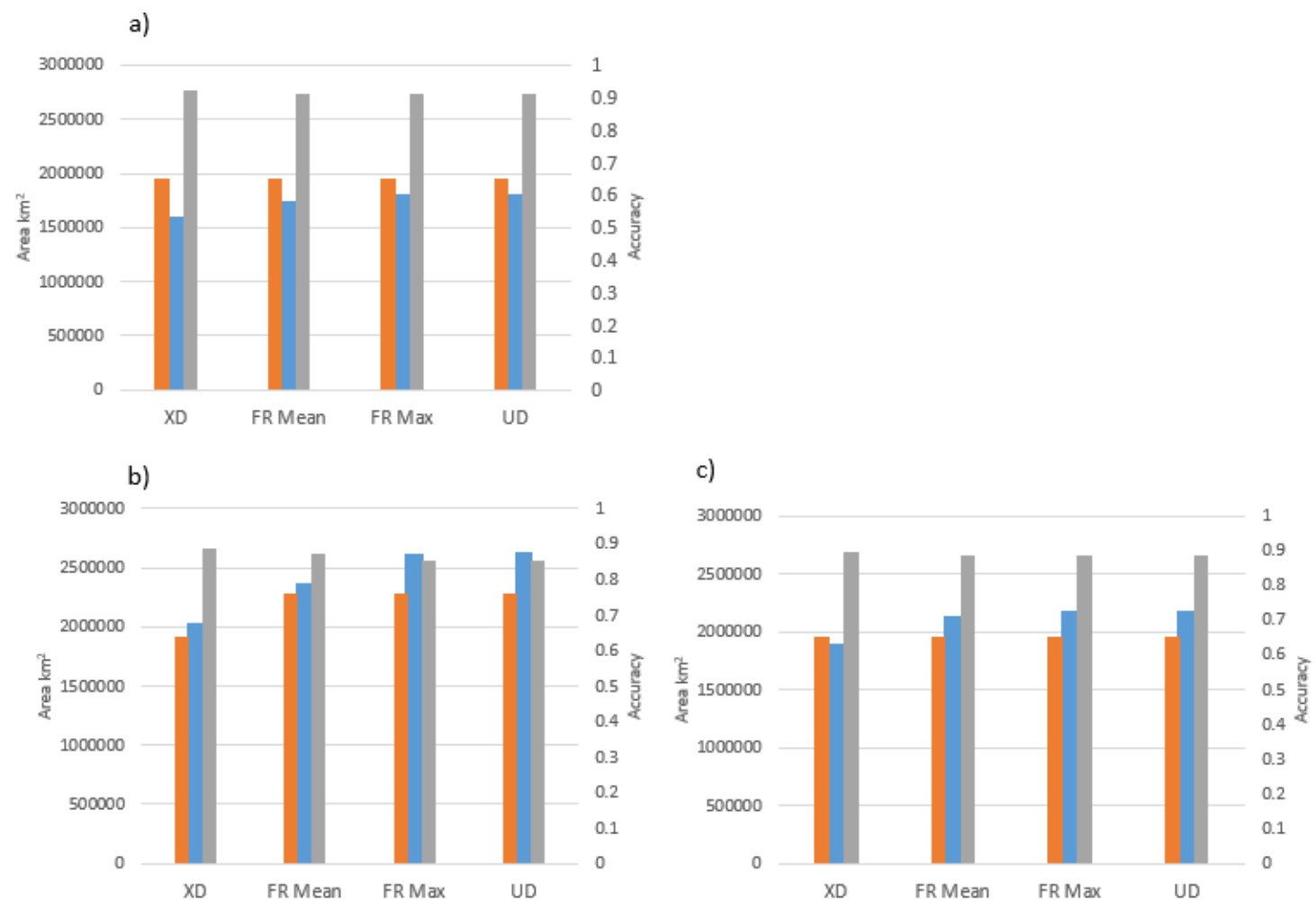

Figure 6.3: Areal extent (blue), sensitivity (orange) and specificity (of random background points - gray) of different dispersal models for a) Scenario A, b) Scenario cBAM, c) Scenario dBAM.

The results from the different dispersal models were less surprising, agreeing with the findings from Chapters 2 and 3. As the dispersal models ability to incorporate longdistance dispersal was increased (XD to FR Mean to FR Max to UD), so too was the area predicted as present (Figures 6.2, 6.3). For scenarios A and ABAM, both dispersal models FR Max and UD predicted exactly the same area as present, suggesting that all habitat was considered accessible if the maximum natal dispersal distance is achieved each year. A change in dispersal model resulted in differences across all implementations of cBAM (in terms of both area and accuracy), in part because the potential area predicted present was larger, meaning dispersal models had the ability to restrict projections more so than in scenarios A or dBAM. 
Sensitivity was highest for the cBAM scenarios with the exception of no dispersal. Conversely, no differences were observed in sensitivity between A and dBAM scenarios. Specificity was calculated by generating 500 random background points. Therefore, this should be considered with caution, as this metric identifies the models ability to correctly predict random points (and not species absence). However, it does act as a proxy to illustrate the differences in area predicted present, with a 'careful' assumption of absence. As expected, scenario A (with the smallest predicted area) scored the highest specificity, followed by dBAM and then cBAM (Figure 6.3).

Uncertainty among projections was calculated using ensemble models and the Ensemble Agreement Index (EAI) described in Chapter 2. It can be seen from an ensemble model of all 12 combinations of projections (three BAM scenarios and four dispersal models), that there is moderate uncertainty of oilbirds projected into the future (Figure 6.4), with an Ensemble Agreement Index (EAI) of 0.715. In particular, there is increased uncertainty in the south-east of the projected distribution. When uncertainty was quantified for the three BAM scenarios (Figure 6.5) compared to the four dispersal models (Figure 6.6), it can be seen that the majority of the uncertainty arises from the BAM scenarios. From Figure 6.5, it can be seen that for each of the dispersal models, the areas projected as present by the three BAM scenarios differ. A large area of the southeast and south-west is projected as present by only one BAM scenario, with other areas of disagreement on the outskirts of the core distribution in the north of South America. In comparison, differences between the dispersal models are relatively minimal, with differences again observed mainly in the south-east, but in general there is consensus among the dispersal models. 


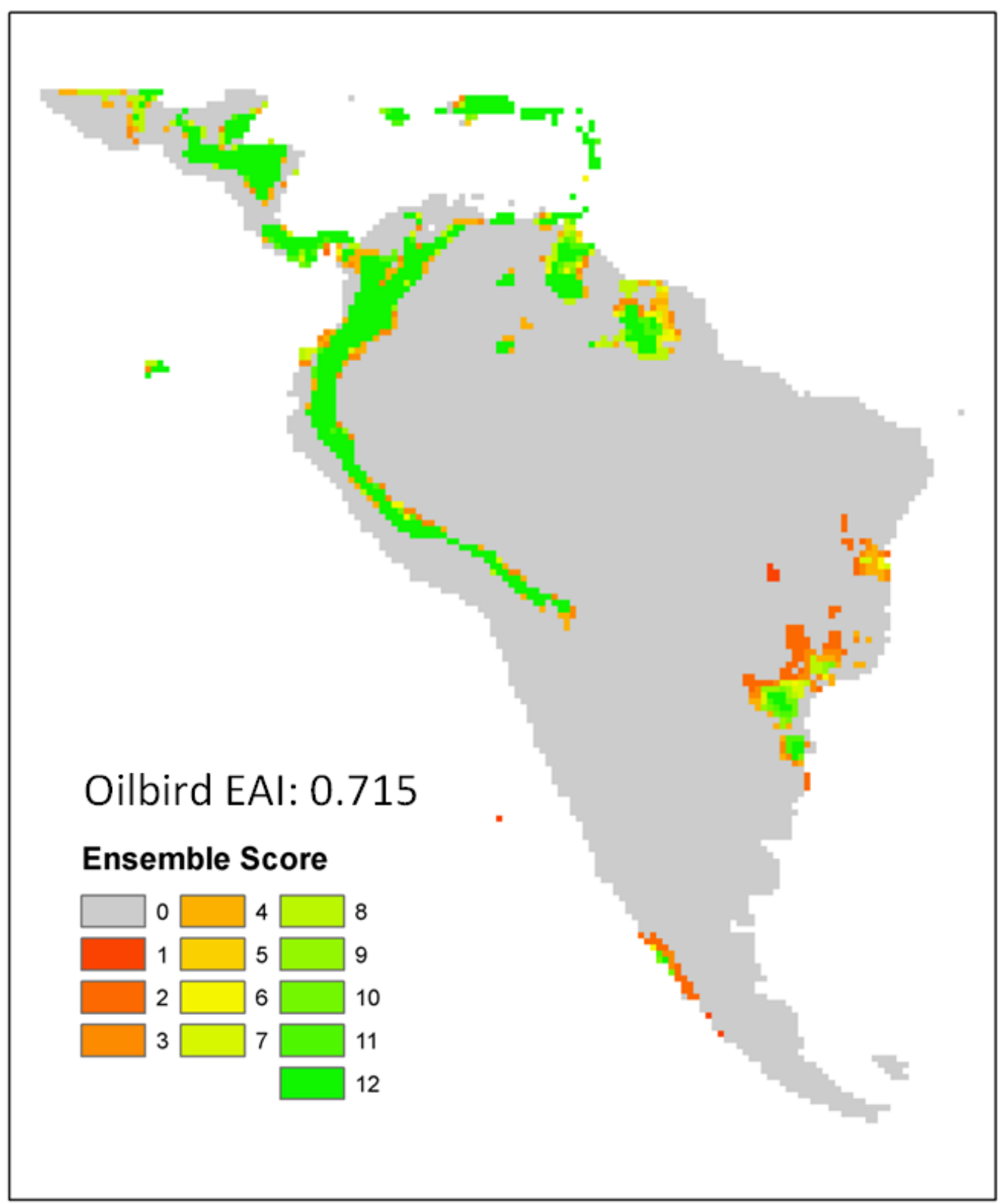

Figure 6.4: Ensemble model of 2010 oilbird distribution based on three BAM scenarios and four dispersal models. 

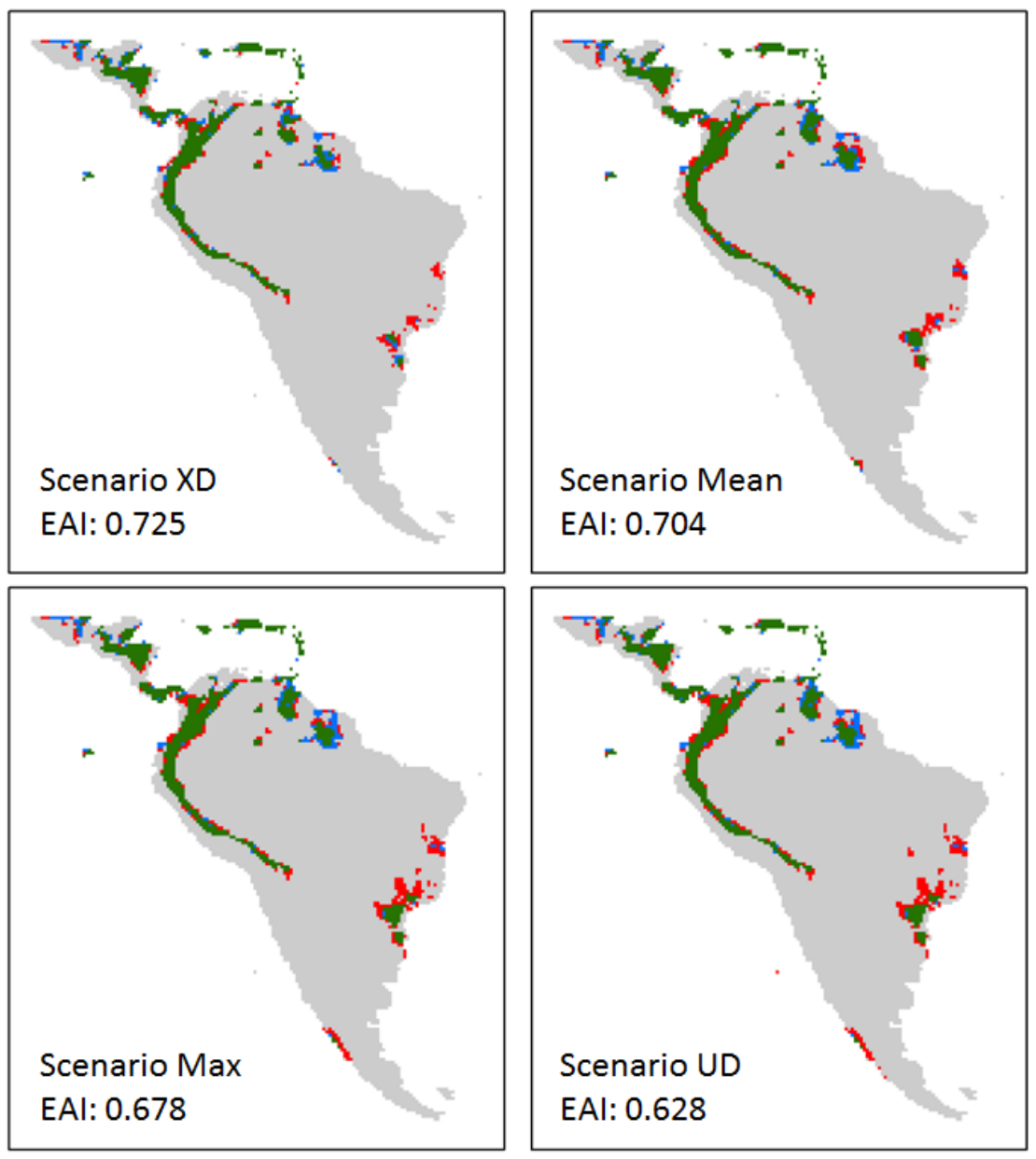

\section{Ensemble Score}

$\square$
$\square$
$\square$
$\square$
2
3

Figure 6.5: Ensemble models and Ensemble Agreement Index (EAI) for the three BAM scenarios separated by dispersal model 

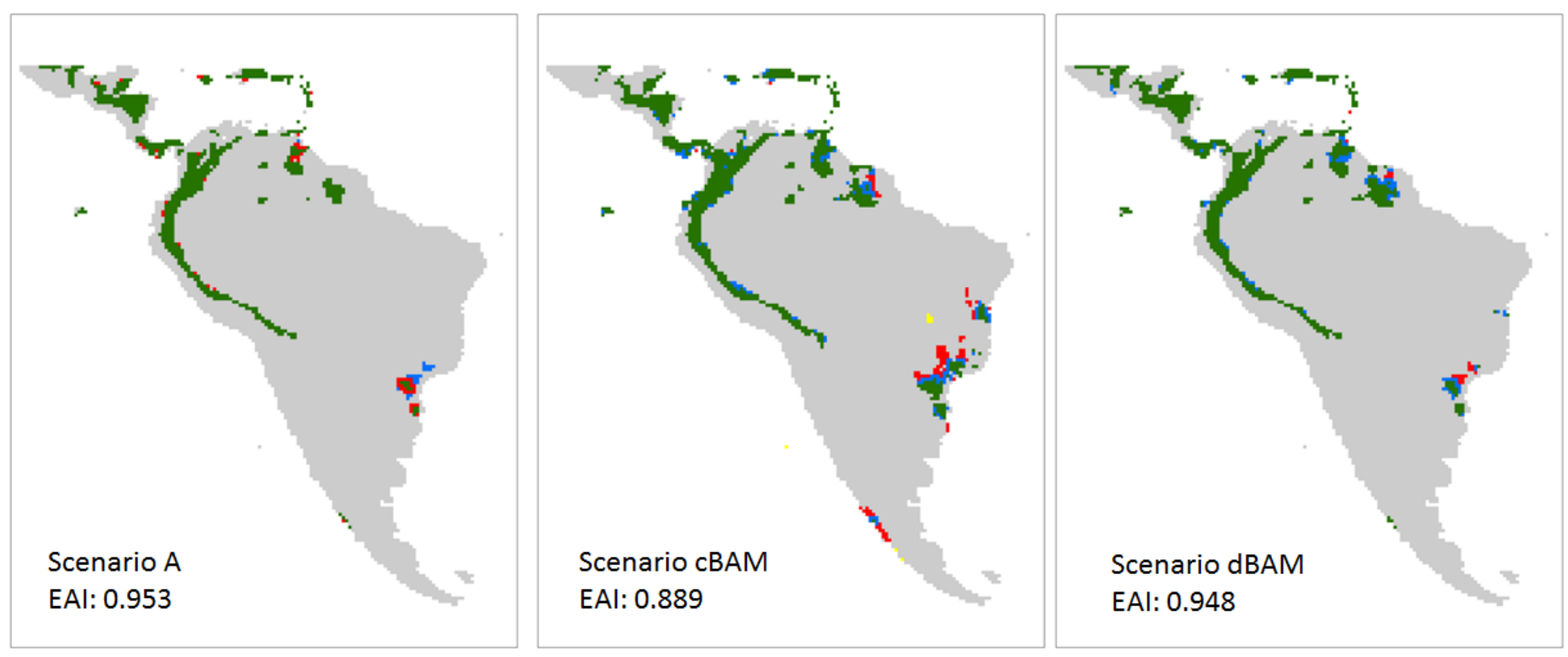

\section{Ensemble Score}

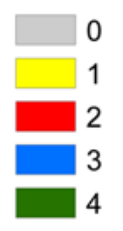

Figure 6.6: Ensemble models and Ensemble Agreement Index (EAI) for the four dispersal models separated by BAM scenarios 


\section{DISCUSSION}

While broad temporal movements of species occur in response to changes in climate, the cumulative result of finer scale regular movements such as foraging excursions can significantly alter the accessibility of future habitat. Furthermore, regular movements are highly dependent on contingent landscape variables which operate at a distinctly finer spatiotemporal scale than climate change. Therefore, the coupling of any statistical and dispersal model has to incorporate both climate and landscape variables due to their profound influence on determining distributional patterns; however the scale at which these processes occur is an important element in determining the amount of area considered suitable and accessible.

Factors that are found to be important at one scale frequently prove to be less so at another scale (Turner et al. 1989). Due to the inherent scale trade-off between resolution and extent, changes in oilbird distributions across the continent of South America were investigated at a $50 \mathrm{~km}$ resolution. This decision was made in part due to the coarse scale of the climate data, as well as the intensive computing power that would have been needed to simulate the dynamic movement-resources model at a focal neighborhood for every $10 \mathrm{~km}$ observation across the continent (as was conducted in Chapter 5 for Venezuela). Differing results from Chapter 5 were therefore expected, and the static representation of $\mathbf{B}$ and $\mathbf{M}$ (cBAM) outperformed both $\mathrm{A}$ and $\mathrm{dBAM}$ in terms of sensitivity (Figure 6.3). While this contradicts the findings that the incorporation of a dynamic movement-resources model improved both the accuracy and ecological realism in Chapter 5, there are a number of reasons this result could have been observed.

The spatial resolution of the abiotic factors meant that the variation in oilbird and vegetation distributions were less pronounced, causing the differences in habitat 
fragmentation, connectivity, and sustainability, and any subsequent patterns caused by fine-scale regular movements to be obscured. Changes in land cover occur more frequently than climate, and while land cover data was available from MODIS for 2001 and 2010 (LP DAAC 2010), the change in distribution of vegetation species was difficult to ascertain due to the manipulation of CRU and WorldClim data. While manipulation was necessary due to the lack of available temporally explicit fine scale climate data, several assumptions in data processing had to be made, which subsequently influenced vegetation distribution patterns, resulting in decreased spatial variation in the distribution of plant species. Furthermore, differences between abiotic and biotic layers between 2001 and 2010, were minimal. Therefore, variation in the distributions resulted from the differences in species observations pre- and post-2000 which were used in model calibration.

The decision to project plant species using observations post-2000 instead of extrapolating the pre-2000 species-environment relationships was taken so that changes could be based on 'observed' differences. However, these changes could be an artefact of continental data coverage rather than actual differences in the species distributions. Therefore, due to these limitations in generating biotic resources, differences from Chapter 5 were observed. The calibration of future distributions on both abiotic and biotic factors (e.g. climate and presence of food species) is a relatively uncommon method within SDM, meaning that limitations of projecting species distributions on both $\mathbf{A}$ and $\mathbf{B}$ identified in this study should serve to inform future researchers.

The accuracy metrics discussed from Chapters 2 and 3 could not be used due to the fact that presence-only test data was available. Similarly, due to the need to threshold the suitable habitat in order to couple the statistical and dispersal models, the metrics implemented in Chapter 5 could also not be used. Therefore, sensitivity was the only 
metric which could be calculated, and overall this was relatively low (0.64 to 0.76). However, these results are not out of context with results reported from this dissertation, with most future projections reporting lower than expected accuracy, in part due to the increased uncertainty in projections previously identified such as feedbacks, interactions, and non-analog climates. The use of sensitivity as a sole accuracy metric should be treated with caution, as results from the previous chapters have identified scenarios where sensitivity was high, but when specificity was considered alongside, accuracy and any subsequent inferences changed. In other words, sensitivity only measures half of a model's accuracy, and any judgements based solely on sensitivity should proceed with caution. In fact, much of the justification for integrating regular movement within SDM is to remove false positives created by models which over-predict (Figure 6.1). Therefore, some measure of absence should ideally be incorporated. While a measure of specificity was calculated from random background points, this should be interpreted with caution and only used to illustrate potential specificity.

While projections of global climate change are well established (Hijmans et al. 2005), changes in land cover are highly dependent on local processes and operate across much shorter temporal scales, meaning continental projections for 2100 or 2050 either do not exist, or they are highly subjective to a number of uncertainties. Future changes in regional scale land covers have been projected, but land change science is itself an active research discipline with multiple frontiers, and the generation of a continental land cover map would itself be the results of years of research. That is not to say that climate change is not subject to many of the same issues, just that global datasets of land cover are not currently available due to the increased number of driving factors causing land cover change, as well as the fact that many of the drivers of land cover change operate on shorter temporal scales that add further uncertainty into projections. 
Despite the conceptual and methodological uncertainties identified, the use of multiple scales of movement in SDM remains promising. Advancements in data collection and management are improving, and new climate and land cover projections continue to emerge. The oilbird has an extensive range across South America, meaning that any future changes in its distribution had to be studied at a continental scale. However, many other species do not inhabit such a vast geographic area, meaning projections can be calibrated at finer spatial scales, whereby more information regarding connectivity and accessibility can be explored using focal statistics, and land cover projections are conducted at a finer spatial extent reducing the uncertainties and increasing the possibility to project into the future. Regardless, the use of multiple scales of movement analysis and explicit consideration of all three BAM factors is novel, and should foster future research. As stated, projecting into future time and space is not without uncertainties, and many of the results in this final chapter pose more questions than they actually answer. For example, what is the correct spatial scale to study both regular movements and dispersal? How should the differences in temporal rate of land cover and climate change be accounted for? Are accuracy assessments of future projections (or even current projections) even possible with current metrics, and if so how should we interpret the evaluations? The findings from this final research chapter should aid future researchers aiming to combine multiple scales of movement within distribution models, and at the very least develop discussion surrounding the need for more multiscale analysis in SDM. 


\section{CHAPTER 7: INCORPORATING MOVEMENT IN SPECIES DISTRIBUTION MODELS: A CONCLUSION}

\section{INTRODUCTION}

Species distribution models are one of the most important GIScience research areas in biogeography and are the primary means by which the drivers of current and future geographic distributions of species are investigated. Species distribution modelling has recently 'exploded' within the context of conservation biogeography (Franklin 2010), and continues to increase in popularity with researchers, conservationists, and government officials. These models span several disciplines as is evident by the variety of journals identified in Chapter 1. SDMs have become particularly important for testing biogeographical hypotheses, assessing species invasion risk, as well as predicting the impact of climate change on species distributions. The use of the 'BAM' framework (Figure 1.1) within the SDM discipline to identify the factors that drive the geographic distributions of species is becoming increasingly popular. Within this framework, movement factors that refer to the processes that lead to an area being accessible (e.g., via dispersal or regular movements) are an important yet understudied component. The importance of incorporating movement within SDM has been identified by several researchers (Soberón and Peterson, 2005; Franklin 2010; Miller and Holloway 2015), as well as continually throughout this dissertation. Results from the meta-analysis identify that the consideration of movement in SDM is increasing, with a large number of studies published in recent years (Figure 1.2). However, movement is a diverse and complex process that influences species distributions across a range of spatiotemporal scales, meaning how movement is conceptualized, modelled and evaluated can have large implications for inferences made from SDM projections. Within this dissertation, I have 
identified several key research questions related to movement in SDM, pertaining to dispersal in response to climate change, regular movements of mobile species, and the need to consider movement beyond a single scale. This research should therefore act as a timely addition to this burgeoning research frontier.

\section{DisPersal ModelLing}

The importance of incorporating dispersal alongside SDMs projecting into the future cannot be overstated, as the results of SDMs which ignore dispersal are subject to high levels of uncertainty. Various methods of incorporating dispersal alongside the statistical model of species-environment relationships have been proposed (Table 1.4), but to date the differences among dispersal models have not been investigated. Chapters 2 and 3 are therefore the first studies that have extensively compared the accuracy and uncertainty of the methods of incorporating dispersal in SDM, and as such should serve as a foundation for studies projecting into future climates.

While some comparisons between models have been made (e.g. Engler and Guisan, 2009; Sullivan et al. 2012; Cunze et al. 2013), discussion has focused on the differences in areal extents projected by the models. While this is important to identify the uncertainty of future projections, a question I kept returning to was 'which dispersal model is the best'? Therefore, an early aim of this research was to apply an accuracy metric to these dispersal models. The use of past data to calibrate and extrapolate speciesenvironment relationships to the current time-period provided this research with the possibility to evaluate dispersal models. The use of breeding bird atlases from Great Britain, provided Chapter 2 with comprehensive, reliable, and accurate datasets for two time periods (1990 and 2010). This provided the unique possibility to assess dispersal model accuracy, something that has not been achieved before. 
Many of the methods of dispersal differed greatly in terms of their accuracy, particularly between the restrictive and the expansive methods, while other methods of dispersal did not differ (e.g. discrete kernels and unlimited dispersal). The use of no dispersal (XD) yielded significantly lower accuracy for metrics which reward correctly predicting presences (e.g. TSS) when averaged across the 50 species, while conversely also recording the highest scores for the accuracy metrics which favor correctly predicting absences (Figures 2.2 and 2.3). Subsequently XD should only be used if the researcher has a valid reason to do so, and even then other simulations of dispersal should be incorporated alongside this. The use of limited dispersal (L1 or L2) resulted in higher accuracies for invasive species that were not in equilibrium with their environment in 1990, and subsequently had more geographic area to disperse into. Finally, the use of a probabilistic kernel did well to incorporate long-distance dispersal while controlling the extent of the geographic area to which this was applied to by probabilities.

As accuracy assessments of future projections of species distributions are not possible, differences in areal extents predicted as present were also evaluated. Results identified that a slight change in the implementation of a dispersal model caused substantial areal differences (Table 2.5). The development of the Ensemble Agreement Index allowed for the quantification of uncertainty among the different dispersal models, and will provide researchers with a quantitative tool to assess the variations in their dispersal models as well as for other areas of uncertainty in SDM (e.g. use of statistical method, threshold classification) or any environmental or geographic research where the main output can be binary (e.g. flood risk, urban growth). The results from Chapter 2 identified significant differences in both the accuracy and uncertainty of dispersal models applied for British birds. Whether these results would replicate across different spatial 
and temporal scales and different taxa was an obvious progression from this research, and led directly into Chapter 3.

Fossilized pollen data has been used to hindcast species-environment relationships into the Quaternary, meaning evaluation of dispersal models could also be undertaken for plant genera in North America. A key finding of Chapter 2 was that the different theoretical dispersal kernels greatly influenced the results obtained. Therefore, an aim of this chapter was to derive empirical dispersal kernels, thus eliminating this source of uncertainty. The production of the dispersal kernels for North America flora (Figure 3.2, Table 3.2, and Appendix 4) will provide researchers with a database with which to derive empirical dispersal distances of North American flora based on dispersal vectors and species traits.

Results from Chapter 3 indicate that the assumption of homogeneity in abiotic suitability of locations between current and future time should be revisited. Many dispersal models implement dispersal from current time $\left[\mathrm{t}_{1}\right]$ to future time $\left[\mathrm{t}_{2}\right]$, assuming that the habitat at intermediate time periods is also suitable. However, substantial differences were found in abiotic suitability across intermediate time-periods, resulting in significant differences of accuracy and area for future projections. In particular, the differences in abiotic suitability for $4 \mathrm{ka} \mathrm{BP}$ and $3 \mathrm{ka} \mathrm{BP}$ were obvious (Figure 3.6), with dispersal models (e.g. L1) reporting different accuracies due to the reduced area of abiotic suitability at 4ka BP (Figure 3.2).

Similarly, how scale influences these dispersal models should also be a priority for researchers implementing these models. Dispersal distances were relatively small compared to the coarse resolution of the SDM, resulting in simulated dispersal distances much greater than expected when dispersal was simulated as multiple time-steps. Methods such as fixed rate (FR) and the discrete dispersal kernel (DK) over-fitted the 155 
dispersal ability of many species due to the assumption of homogeneity within the presence grid (Figure 3.5). These results were a direct function of the scale of the data used, and researchers need to be aware of how these will impact future projections. By deconstructing genera based on dispersal vector type, the next stage of this research is to identify ecological insights provided by the dispersal models.

For the first time in SDM research, we have been able to compare these dispersal models based on how well they predict future geographic distributions. The inclusion of the dispersal models as $\mathrm{R}$ functions in Appendix 2 will provide future researchers with the means to readily compare implementations of dispersal models, ultimately improving any inferences made from future projections of species distributions. Not only has this research provided practitioners with a product with which to select a dispersal model to use, saving their time, resources and research efforts, it has also resulted in a better understanding of the effects of both the accuracy and uncertainty on projected distributions, extinction rates and dispersal patterns. Dispersal modelling should now be a compulsory aspect of any SDM extrapolating species-environment relationships across time or space, and these studies have added a timely addition to the growing literature surrounding the uncertainty among model selection.

\section{REGULAR MOVEMENTS}

Fine scale regular movement behaviors such as home range maintenance, foraging, and diurnal migrations have yet to be regularly incorporated within SDM, despite the important role they play in the maintenance of a mobile species distribution. Moreover, these movements do not occur independently of biotic factors, and the dependence of animals on food plants (biotic resources) can be an important biotic determinant of species distributions. While some studies have used focal statistics describing the 
contingent land cover features of a neighborhood as a proxy for regular movements (e.g. Ashcroft and Major 2013), the dynamic relationship between biotic factors and regular movement has yet to be studied. Chapter 5 identified how a Lagrangian approach to movement and spatial simulation could be used to investigate the movement of species across large areas, in this case oilbirds, realistically representing the dynamic relationship between biotic factors and movement. From this, an environmental layer which represented the sustainability of a neighborhood, based on connectivity, accessibility, and sustainability of biotic resources was created.

At a spatial scale of $10 \mathrm{~km}$ in Venezuela, and a focal area of $50 \mathrm{~km}$, the use of this dynamic layer improved the accuracy and ecological realism of SDM projections compared to other widely applied BAM scenarios (Table 5.3, Figure 5.3). In particular, the use of a static variable representing biotic factors and movement appeared to overpredict the distribution (Figure 5.3), and it can be seen that the layer developed to represent the dynamic relationship between biotic factors and movement provided more information in the variability of vegetation distribution and accessibility (Figure 5.4).

The explicit simulation of movement represented by individual movement paths in SDM is novel. No previous research has integrated a Lagrangian approach of movement in SDM using the finest scale representation of movement (i.e. movement paths). The Lagrangian approach has been supported by ecologists and modelers (Tang and Bennett 2010) as it identifies population phenomena by studying the individual, thus describing population patterns based on detailed information of individual movement processes. The results from Chapter 5 therefore identify the potential for researchers to study movement as a Lagrangian approach with concepts and methodologies from movement ecology and movement pattern analysis (MPA) within SDM. 
The use of step-selection function (SSF) as an input into the spatial simulation is also novel, and subsequently adds to the research and discussion in the aforementioned fields. Moreover, the development of the spatial simulation model (available in Appendix 6) will provide future researchers who are aiming to simulate movement based on SSF parameters the platform with which to do so in a dynamic modelling environment (e.g. NetLogo).

SSF of oilbird movement was estimated using landscape features, biotic resources, and bird memory, and the results demonstrated realistic movement patterns (Figure 5.2). Furthermore, the use of an interaction term between memory and biotic resources improved the final SSF model (Table 5.2). The incorporation of memory in SSF has been suggested as a necessary input (Fagan et al. 2013); however, to date only one study has acknowledged this (Oliveira-Santos et al. 2016). The results from Chapter 5 highlighted the need to measure spatial memory of biotic resources compared with just spatial memory, and aided in the interpretation of how oilbirds were selecting movement steps, and should inform future SSF studies.

Prior to model building, several questions regarding SSF arose. This was the basis for Chapter 4, and this study found that differences in a number of user-decisions influenced the models results. These decisions had direct impacts on how SSF could be simulated. For example, an increase in the number of available steps significantly increased the processing time of the spatial simulation. While a really low (2) or high (200) number of steps potentially under- and over-sampled the landscape features respectively, few differences were found between medium numbers (Tables 4.2, 4.3). This in turn informed the use of 5 available steps in the oilbird SSF. Idiosyncratic preferences had the strongest influence on the results observed, while averaging coefficients across individuals resulted in higher values when studied at an aggregate 
level, indicating that individual preferences are lost when studied solely at an aggregate level. This research directly answered specific questions in the development of SSF for this dissertation, and allowed decisions to be made with the appreciation of any uncertainty in the results. User-decisions of SSF practitioners should subsequently be justified based on specific research objectives and where possible exploration of userdecisions should be conducted. Further research into other user-decisions of SSF (e.g. how memory is modelled and incorporated in the statistical model) should continue.

\section{Multi-Scale Movements}

In spite of research that has demonstrated how each of these types of movement is important in SDM projections (Chapters 2, 3, and 5), incorporating movement across multiple scales has not been addressed in SDM research (Chapter 1). Components at fine scales can interact and accumulate across space and time, producing patterns and processes at broader scales (Peters et al. 2007). Therefore, the emergent properties of regular movements (such as foraging and habitat connectivity) can effectively alter the dispersal outcomes of populations which are responding to changes in climate (Heffernan et al. 2014). The aim of the final chapter was to combine both movement processes in order to improve the accuracy and interpretation of the oilbird distribution across a 10 year period for the continent of South America.

Unfortunately, due to the inherent scale trade-off between extent and resolution, many of the results identified from the previous chapters were obscured due to the coarse resolution. At this spatial resolution, the static representation of $\mathbf{B}$ and $\mathbf{M}$ appeared to outperform the dynamic scenario, with the sensitivity for the cBAM models higher (Figure 6.3). However, an unassailable aspect of this final chapter was assessing the accuracy. Due to the necessary model building phases across multiple movement models, 
only sensitivity (and specificity of random background points) could be assessed. New evaluation metrics for presence-only data remain a research frontier in SDM (Franklin 2009), and the results from this dissertation have identified new problems in model evaluation that need to be addressed.

Subsequently, the Ensemble Agreement Index was able to quantify uncertainty in oilbird projections. Moderate uncertainty was recorded between the three BAM scenarios (Figure 6.5), while low uncertainty was recorded between the four dispersal models (Figure 6.6). Reasons for high uncertainty between BAM scenarios were identified due to the coarsening of the spatial scale (resolution and extent), and with much of the detail in the regular movements for animals lost. The reasons for uncertainty between dispersal models were discussed at length in Chapters 2 and 3, and the results from Chapter 6 support these findings. Furthermore, the use of four dispersal models does well to capture the uncertainty between the extreme approaches, meaning that the dispersal potential of the oilbirds is not restricting accessibility to future (abiotic and biotic) suitable habitat, but rather it is differences in BAM variables used in the statistical model. The findings from this final research chapter should foster future researchers aiming to combine multiple scales of movement within SDMs, and at the very least develop discussion surrounding the need for more multi-scale analysis in SDM.

\section{FUTURE DIRECTIONS}

Incorporating movement should now be a compulsory aspect of any study projecting the current or future distributions of species. This dissertation has investigated and reported results across a broad range of taxa, for multiple movement behaviors at a range of spatial and temporal scales. The results from this dissertation have identified a number of key findings pertaining to dispersal in response to climate change and regular 
movements, as well as opening a dialogue regarding the incorporation of multiple scales of movement in SDM research. This dissertation concludes with the identification of a number of possible future research trajectories for the continued incorporation of movement with species distribution modelling:

1. The use of empirically derived dispersal kernels for the North American flora allowed for a discussion of the effectiveness of the dispersal models at projecting distributional shifts as well as the potential to investigate the ecological importance of dispersal as a driving factor. This is in contrast to the lengthy discussion on the inherent uncertainty in the choice of theoretical kernel describing British bird dispersal distances. Future research should therefore continue to strive to collate empirical information on dispersal distances, and new methods to generalize this information to a wider range of species groups are needed.

2. The assumption of abiotic homogeneity over time should be revisited. The results from Chapters 1 and 2 highlighted significant differences between all implementations of models which simulated dispersal as a one-step process and as a multiple-step process. Research should be directed towards further exploring this fundamental issue, focusing on how often the temporal period should be deconstructed and if data coverage allows, the accuracy of intermediate timeperiods could be evaluated in order to identify whether errors in these models propagate.

3. How does scale (in terms of both resolution and extent) influence how movement is incorporated in SDM? Scale arose as a discussion point in every chapter, and as such warrants further exploration. For example, differences between the dispersal 
distances estimated for North American flora and the resolution of the projected distributions resulted in dispersal capacity being over-estimated six-fold. This is a serious issue that could have important ramifications for any study extrapolating across time or space.

4. This dissertation primarily focused on birds and plants due to the abundance of data regarding species observations and their movements. This allowed detailed methods to be developed and evaluated. The exploration of how movement can be incorporated for a different species groups should continue to be investigated, in particular the less frequently studied taxa (Figure 1.3).

5. What is the most accurate dispersal model? This question persists, in part because the different dispersal models succeeded at identifying different and distinct features of movement in relation to specific applications. For example, limited dispersal appeared to outperform for invasive species, while the probabilistic dispersal kernels improved the specificity of birds and plants due to its ability to control long-distance dispersal with probabilities. Research should continue to investigate the accuracy of dispersal models across a broader range of SDM applications.

6. New methods of accuracy assessment are needed. Throughout this dissertation, new and unique scenarios of model evaluation were identified. Independent test data with complete spatial coverage for the whole study area are rare. These accuracy metrics appeared to effectively favor the correct prediction of absences, due to the comprehensive test data containing more absence than presence points across the entire spatial extent. Furthermore, the issues identified in Chapter 6 pertaining to presence-only test data with binary outputs, meant that only one accuracy metric out of the ten used throughout this dissertation (sensitivity) could 
be used. Future research should begin to identify new accuracy metrics so that the incorporation of movement into SDM can be evaluated as rigorously as possible.

7. How can spatial simulation continue to address individual-based simulations of animal movement? To date, individual-based models in SDM have focused on the movement of individuals as grids. Research should continue to investigate how a Lagrangian approach using movement paths (as illustrated in Chapter 5) can be incorporated in SDM.

8. For studies investigating dispersal in response to climate change, the predominant methodology has been to couple the statistical model of abiotic and biotic suitability with a dispersal model. Whereas for movement indicative of finer temporal scales (e.g. regular movements), a spatial variable representative of movement has been incorporated in the statistical model. When calibrating the correlative species-environment relationship, the effects of dispersal limitation may be a latent factor included in the model (Schymanski et al. 2013). Subsequently, to what extent does coupling the statistical model with a dispersal model propagate potential bias in prediction? Virtual species with known properties could be used to answer this question and quantify any error or uncertainty.

9. A number of studies have begun using the locations obtained from telemetry data as the response data in SDM (e.g. Gscwheng et al. 2012). Despite the vast potential to use telemetry data within an SDM framework, a number of new conceptual issues related to this data structure, such as precision, autocorrelation, and idiosyncratic preferences have arisen. Various methods have been implemented for data filtering, but to date they all remain untested as to their 
impact on SDM results. How this data influences habitat projections needs to be further explored.

10. Finally, research should continue to focus on integrating multiple scales of movement within SDM. Movement undoubtedly occurs across fine and broad spatial and temporal scales, meaning the most informative models will be those that continue to bridge the gap between scales. Research conducted at regional extents or for virtual species should allow this concept to be more readily explored. 


\section{Appendices}

Appendix 1: Meta-Analysis

Please see electronic Appendix 1 
APPENDIX 2: R FUnCTIONS FOR DiSPERSAL MODELS

noDispersal | Identifies accessible future suitable habitat by simulating no dispersal

\section{Description}

This function simulates dispersal by assuming that only currently suitable habitat is accessible in future projections.

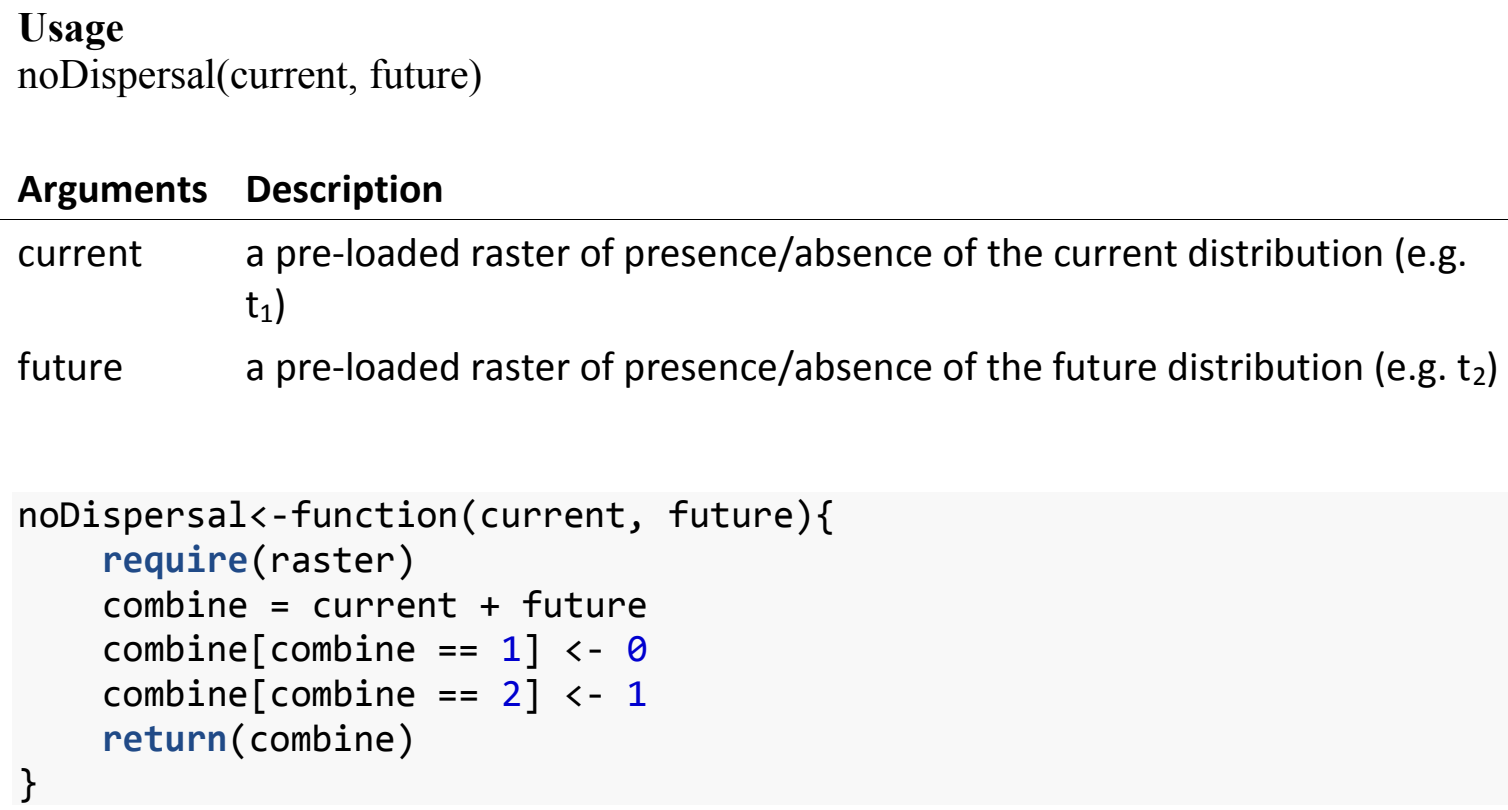


limitedDispersal | Identifies accessible future suitable habitat by simulating limited dispersal

\section{Description}

This function simulates dispersal by assuming that only contiguous areas of suitable habitat in future geographic space that overlap with the areas of suitable habitat in current geographic space are accessible. This can be varied by using different cases of connectivity for the contiguous areas.

\section{Usage}

limitedDispersal(current, future, neigh)

\section{Arguments Description}

current a pre-loaded raster of presence/absence of the current distribution (e.g. $\mathrm{t}_{1}$ )

future a pre-loaded raster of presence/absence of the future distribution (e.g. $t_{2}$ ) neigh condition of connectivity for the future contiguous area: 4 (rook's case) or 8 (Queen's case)

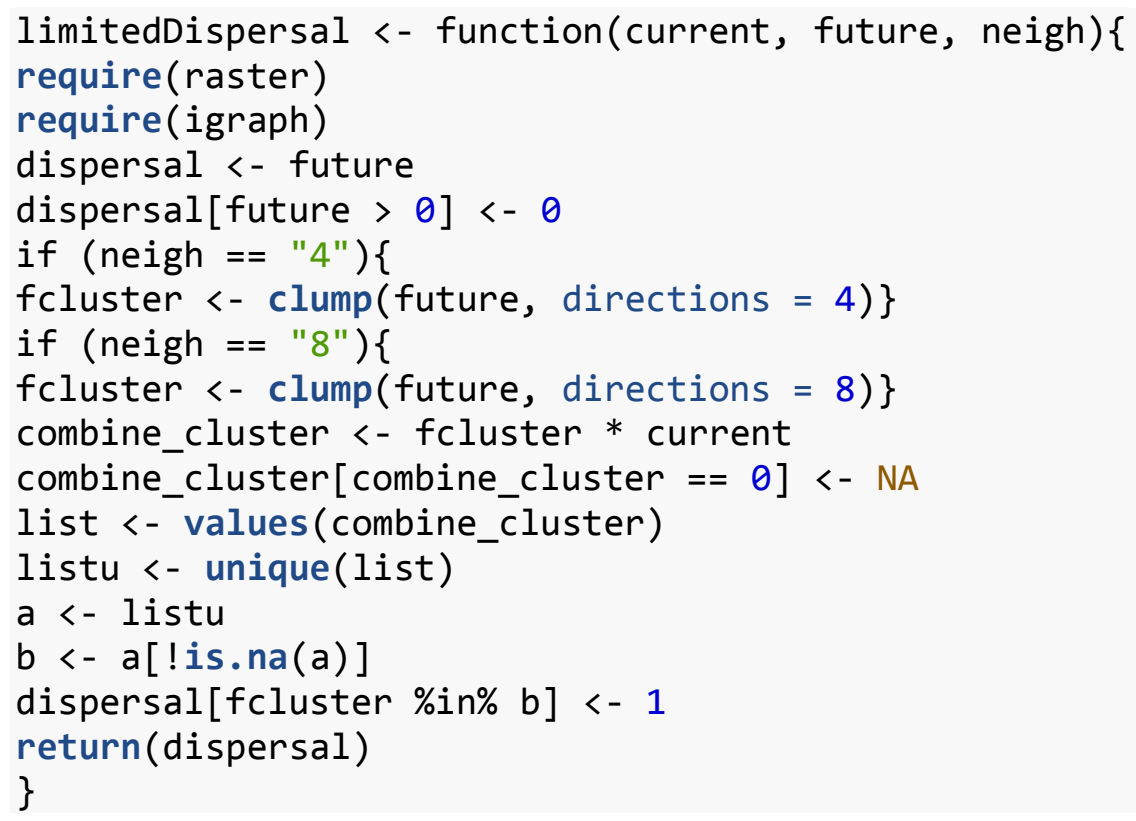


fixedrateDispersal | Identifies accessible future suitable habitat by simulating a fixed rate of dispersal

\section{Description}

This function simulates dispersal by applying a rate of dispersal as a time-based distance square buffer around the current distribution. This function allows the dispersal model to be simulated as a one-step ( $t_{1}$ to $\left.t_{2}\right)$ or as a multiple-step ( $t_{1}$ to $t_{1.5}$ to $\left.t_{2}\right)$ process.

\section{Usage}

fixedrateDispersal(dir, dist, T, A)

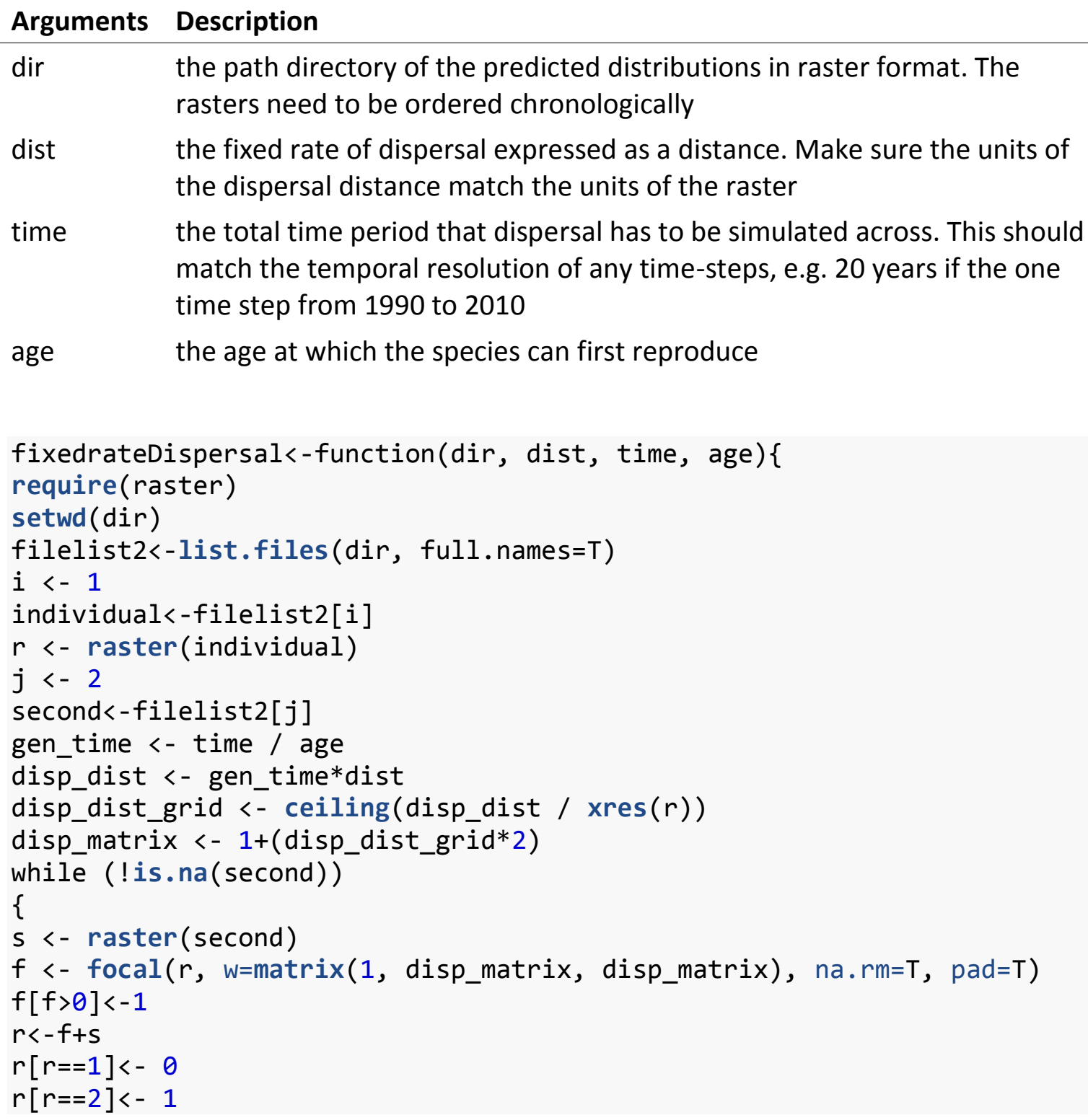




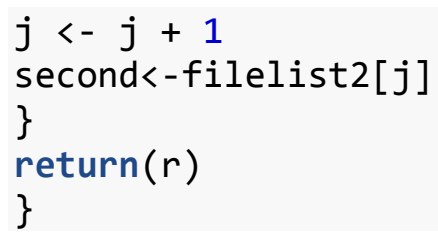


discreteKernel | Identifies accessible future suitable habitat by simulating a discrete dispersal kernel

\section{Description}

This function simulates dispersal by using a discrete dispersal kernel approach.

\section{Usage}

discreteKernel(current, future, dist_vector, R0, time, age)

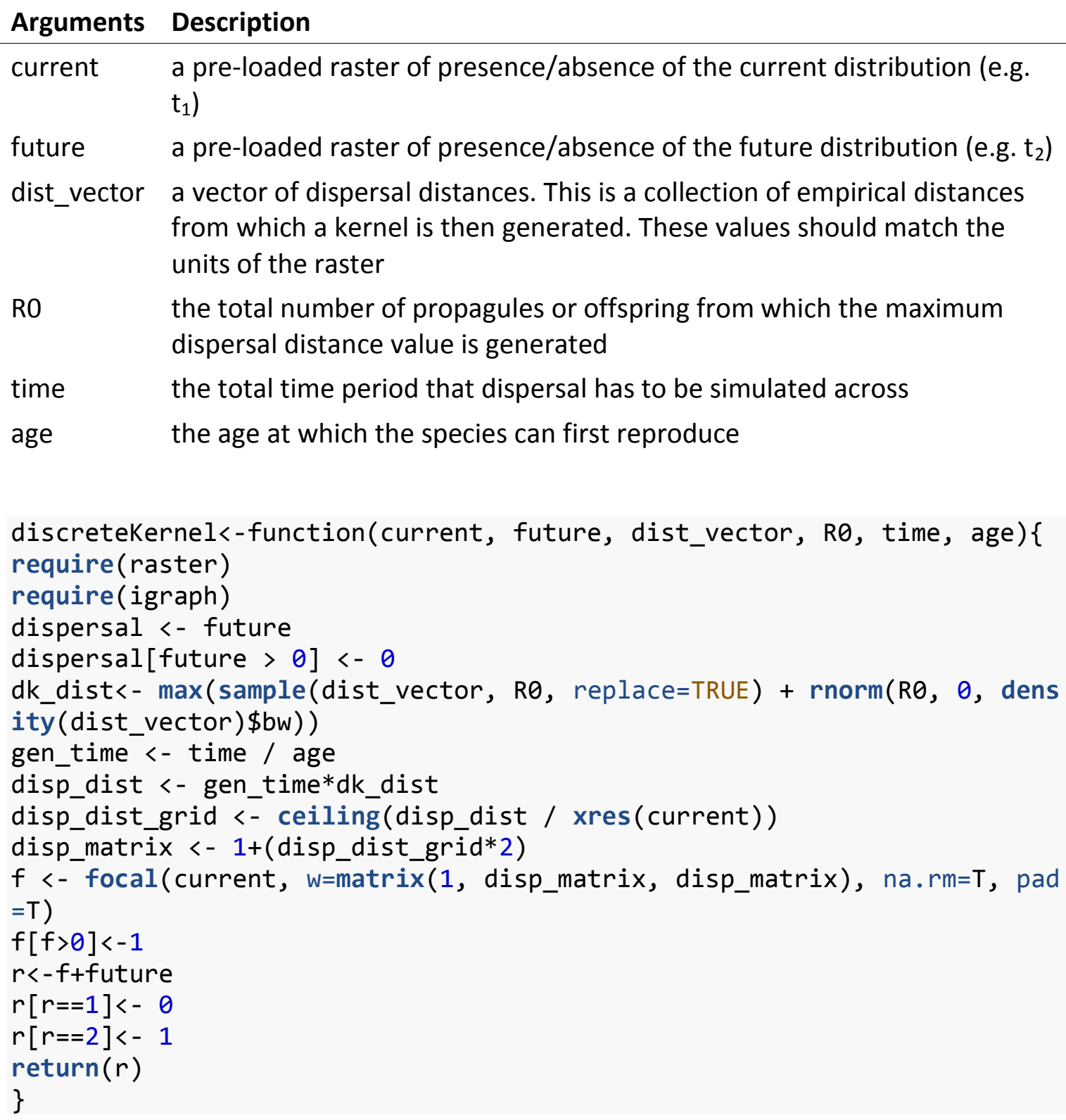


If raw dispersal distances are not available, nor a dispersal kernel, a theoretical dispersal kernel from an estimated mean value and a standard deviation can be derived.

\#\#Create a vector which has the same number as R0 (e.g. 1000)

R日_vector <-seq $(\theta, 1000)$

\#\#generate as many randomly generated values based on an estimated or $\mathrm{d}$ erived mean and sd. Below values are used for example whereby distance is estimated using a Gaussian distribution.

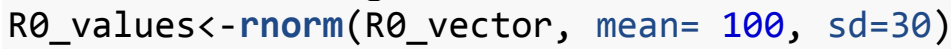

\#\#find the max of these value

maxdist <- $\max \left(R \theta \_v a l u e s\right)$

The following function would then look like this:

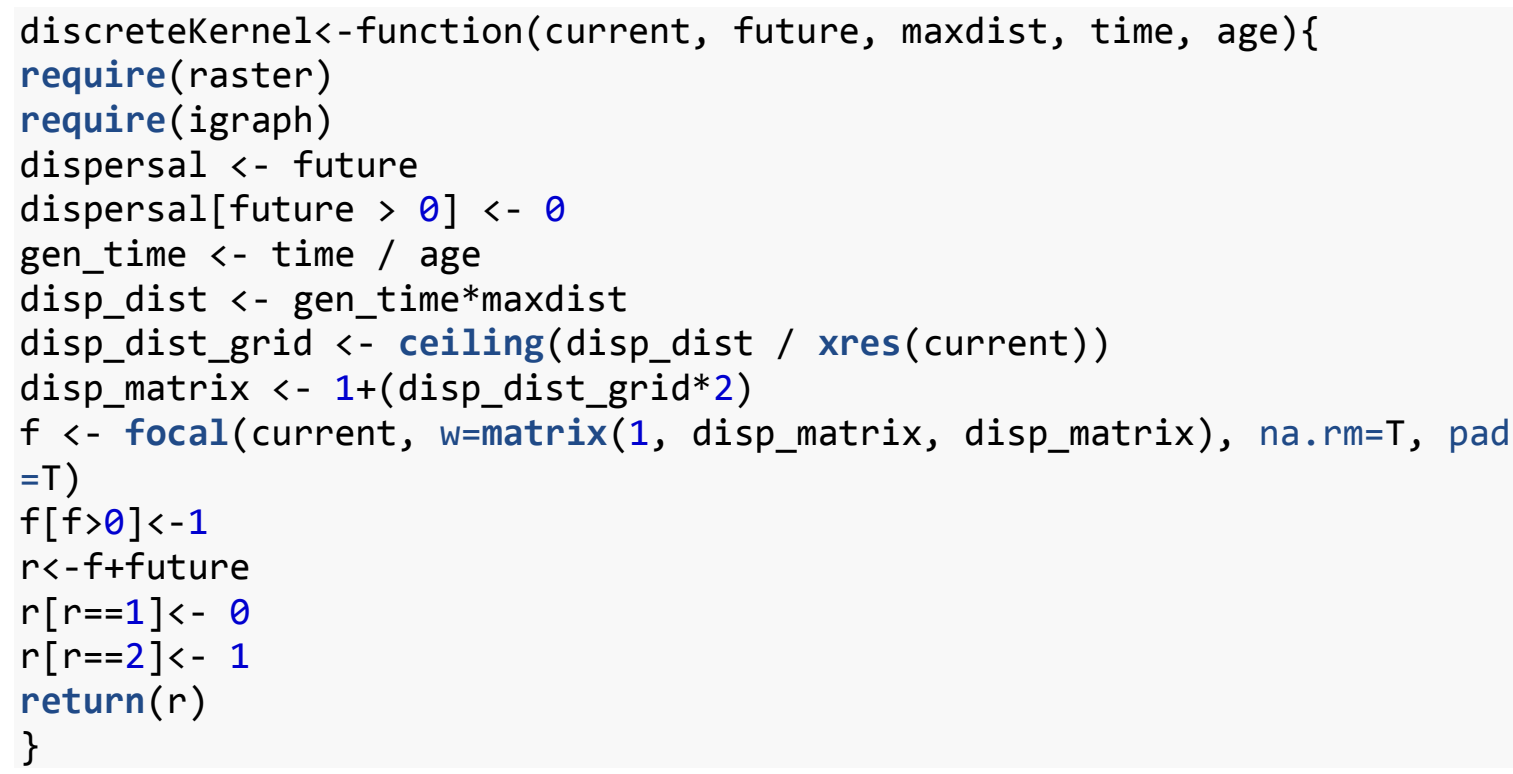




\section{APPendix 3: FinAl Regression Models For Bird DisPersal Distances}

Table A3: Final regression models of the relationship between log body size and both arithmetic and geometric mean natal dispersal distances. Model 1 treats all 50 species as statistically equal, while Model 2 incorporates differences in species dispersal and origin. $* *$ significant at $0.01, *$ significant at 0.05 .

\section{Arithmetic Geometric}

\begin{tabular}{rl|r|c|}
\hline Model 1 & AIC & 114.842 & 110.800 \\
\hline & Intercept & $0.023^{* *}$ & 0.022 \\
\hline Mass & $0.230^{*}$ & $0.226^{* *}$ \\
\hline Model 2 & AIC & 97.521 & 98.426 \\
\hline Intercept & $0.457^{* *}$ & $-0.434^{*}$ \\
\hline Mass & $0.257^{* *}$ & $0.387^{* *}$ \\
\hline Migrant & $0.512^{* *}$ & $0.633^{* *}$ \\
\hline Migrant-Resident & $0.217^{*}$ & 0.102 \\
\hline Introduced & -0.028 & -0.148 \\
\hline
\end{tabular}




\section{APPENDIX 4: DisPersal DistanCES FOR CURRENT NORTH AMERICAN FlORA}

Table A4: Dispersal distance data for North American flora.

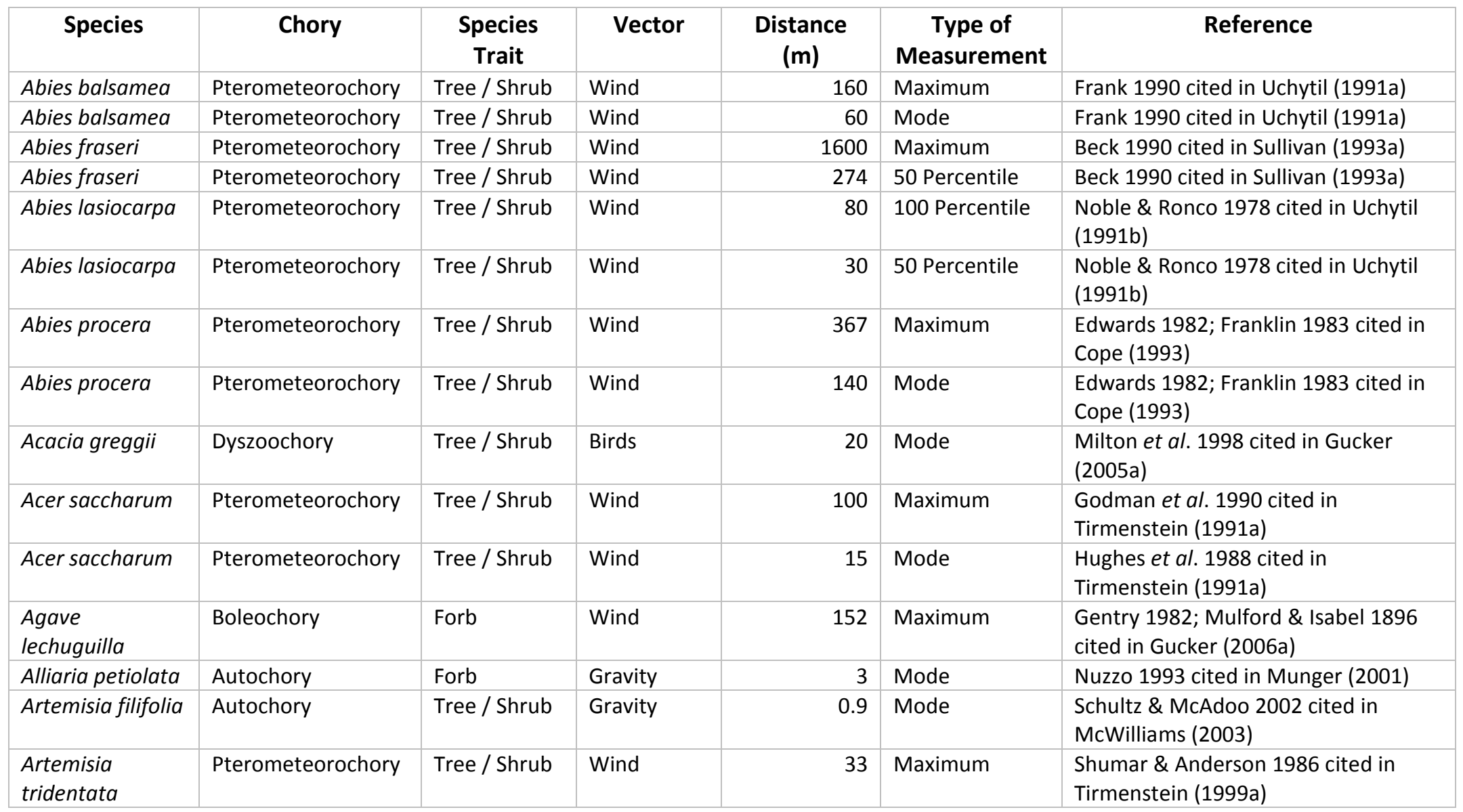


Table A4 (Continued):

\begin{tabular}{|c|c|c|c|c|c|c|}
\hline $\begin{array}{l}\text { Artemisia } \\
\text { tridentata }\end{array}$ & Pterometeorochory & Tree / Shrub & Wind & 9 & 90 Percentile & $\begin{array}{l}\text { Goodrich et al. } 1985 \text { cited in } \\
\text { Tirmenstein (1999a) }\end{array}$ \\
\hline $\begin{array}{l}\text { Artemisia } \\
\text { tridentata subsp. } \\
\text { wyomingensis }\end{array}$ & Pterometeorochory & Tree / Shrub & Wind & 30 & Maximum & $\begin{array}{l}\text { Beetle 1960; Tisdale et al. } 1969 \text { cited } \\
\text { in Howard (1990) }\end{array}$ \\
\hline $\begin{array}{l}\text { Berberis } \\
\text { thunbergii }\end{array}$ & Autochory & Tree / Shrub & Gravity & 80 & Maximum & $\begin{array}{l}\text { Silander \& Klepeis } 1999 \text { cited in } \\
\text { Zouhar (2008) }\end{array}$ \\
\hline $\begin{array}{l}\text { Berberis } \\
\text { thunbergii }\end{array}$ & Autochory & Tree / Shrub & Gravity & 1 & 92 Percentile & $\begin{array}{l}\text { Silander \& Klepeis } 1999 \text { cited in } \\
\text { Zouhar (2008) }\end{array}$ \\
\hline $\begin{array}{l}\text { Betula } \\
\text { papyrifera }\end{array}$ & Pterometeorochory & Tree / Shrub & Wind & 100 & Maximum & $\begin{array}{l}\text { Safford et al. } 1990 \text { cited in Uchytil } \\
\text { (1991c) }\end{array}$ \\
\hline $\begin{array}{l}\text { Betula } \\
\text { papyrifera }\end{array}$ & Pterometeorochory & Tree / Shrub & Wind & 61 & Mode & $\begin{array}{l}\text { Marquis et al. } 1969 \text { cited in Uchytil } \\
\text { (1991c) }\end{array}$ \\
\hline $\begin{array}{l}\text { Calocedrus } \\
\text { decurrens }\end{array}$ & Pterometeorochory & Tree / Shrub & Wind & 60 & 100 Percentile & $\begin{array}{l}\text { McDonald \& Philip } 1980 \text { cited in } \\
\text { Tollefson (2008) }\end{array}$ \\
\hline Carduus nutans & Trichometeorochory & Forb & Wind & 100 & 99 Percentile & $\begin{array}{l}\text { Smith \& Kok } 1984 \text { cited in Zouhar } \\
\text { (2002a) }\end{array}$ \\
\hline Carduus nutans & Trichometeorochory & Forb & Wind & 50 & Mode & $\begin{array}{l}\text { Smith \& Kok } 1984 \text { cited in Zouhar } \\
\text { (2002a) }\end{array}$ \\
\hline $\begin{array}{l}\text { Castillega } \\
\text { cinerea }\end{array}$ & Autochory & Forb & Gravity & 5 & Maximum & Freas 1988 cited in Sclafani (2006) \\
\hline $\begin{array}{l}\text { Ceanothus } \\
\text { cuneatus }\end{array}$ & Ballochory & Tree / Shrub & Ballochory & 10.7 & Maximum & $\begin{array}{l}\text { Biswell \& Gilman } 1961 \text { cited in League } \\
\text { (2005a) }\end{array}$ \\
\hline $\begin{array}{l}\text { Ceanothus } \\
\text { leucodermis }\end{array}$ & Ballochory & Tree / Shrub & Ballochory & 9 & Maximum & Evans et al 1987 cited in Meyer (2011) \\
\hline $\begin{array}{l}\text { Centaurea } \\
\text { solstitialis }\end{array}$ & Trichometeorochory & Forb & Gravity & 0.6 & 92 Percentile & Roche 1992 cited in Zouhar (2002b) \\
\hline $\begin{array}{l}\text { Centaurea } \\
\text { solstitialis }\end{array}$ & Trichometeorochory & Forb & Gravity & 0.3 & 48 Percentile & Roche 1992 cited in Zouhar (2002b) \\
\hline $\begin{array}{l}\text { Cercocarpus } \\
\text { montanus }\end{array}$ & Trichometeorochory & Tree / Shrub & Wind & 140 & Maximum & $\begin{array}{l}\text { Biswell \& Gilman } 1961 \text { cited in } \\
\text { (Gucker 2006b) }\end{array}$ \\
\hline
\end{tabular}


Table A4 (Continued):

\begin{tabular}{|c|c|c|c|c|c|c|}
\hline Cirsium palustre & Trichometeorochory & Forb & Wind & 3000 & Maximum & Nordin 2002 cited in Gucker (2009a) \\
\hline Cirsium palustre & Trichometeorochory & Forb & Wind & 10 & Mode & Nordin 2002 cited in Gucker (2009a) \\
\hline Cirsium vulgare & Trichometeorochory & Forb & Wind & 32 & 90 Percentile & $\begin{array}{l}\text { Klinkamer et al. } 1988 \text { cited in Zouhar } \\
(2002 c)\end{array}$ \\
\hline Cirsium vulgare & Trichometeorochory & Forb & Wind & 27 & 90 Percentile & Randall 2000 cited in Zouhar (2002c) \\
\hline Cirsium vulgare & Trichometeorochory & Forb & Wind & 1 & 50 Percentile & $\begin{array}{l}\text { Klinkamer et al. } 1988 \text { cited in Zouhar } \\
\text { (2002c) }\end{array}$ \\
\hline Cirsium vulgare & Trichometeorochory & Forb & Wind & 2 & 66 Percentile & $\begin{array}{l}\text { Klinkamer et al. } 1988 \text { cited in Zouhar } \\
(2002 c)\end{array}$ \\
\hline Cirsium vulgare & Trichometeorochory & Forb & Wind & 11.6 & Mean & Matlack 1987 cited in Zouhar (2002c) \\
\hline Coronilla varia & Endozoochory & Forb & $\begin{array}{l}\text { Large } \\
\text { Mammal }\end{array}$ & 2000 & Maximum & $\begin{array}{l}\text { Williams et al. } 2008 \text { cited in Gucker } \\
\text { (2009b) }\end{array}$ \\
\hline Coronilla varia & Endozoochory & Forb & $\begin{array}{l}\text { Large } \\
\text { Mammal }\end{array}$ & 568 & Median & $\begin{array}{l}\text { Williams et al. } 2008 \text { cited in Gucker } \\
\text { (2009b) }\end{array}$ \\
\hline Corylus cornuta & Dyszoochory & Tree / Shrub & $\begin{array}{l}\text { Small } \\
\text { Mammal }\end{array}$ & 90 & Maximum & Fryer (2007) \\
\hline $\begin{array}{l}\text { Cynanchum } \\
\text { louiseae }\end{array}$ & Pterometeorochory & Forb & Gravity & 1 & 83 Percentile & $\begin{array}{l}\text { Ladd 2005; Ladd \& Cappuccino } 2005 \\
\text { cited in Stone (2009) }\end{array}$ \\
\hline $\begin{array}{l}\text { Cynanchum } \\
\text { louiseae }\end{array}$ & Pterometeorochory & Forb & Wind & 18 & Mean & $\begin{array}{l}\text { Cappuccino et al. } 2002 \text { cited in Stone } \\
\text { (2009) }\end{array}$ \\
\hline $\begin{array}{l}\text { Cynoglossum } \\
\text { officinale }\end{array}$ & Autochory & Forb & Wind & 1.4 & Maximum & $\begin{array}{l}\text { Boorman \& Fuller } 1984 \text { cited in } \\
\text { Zouhar }(2002 d)\end{array}$ \\
\hline $\begin{array}{l}\text { Cynoglossum } \\
\text { officinale }\end{array}$ & Autochory & Forb & Wind & 0.12 & 75 Percentile & $\begin{array}{l}\text { Boorman \& Fuller } 1984 \text { cited in } \\
\text { Zouhar }(2002 d)\end{array}$ \\
\hline $\begin{array}{l}\text { Cytisus } \\
\text { scoparius, C. } \\
\text { striatus }\end{array}$ & Ballochory & Tree / Shrub & Ballochory & 5.4 & Maximum & Bossard 1991 cited in Zouhar (2005a) \\
\hline
\end{tabular}


Table A4 (Continued):

\begin{tabular}{|c|c|c|c|c|c|c|}
\hline $\begin{array}{l}\text { Cytisus } \\
\text { scoparius, C. } \\
\text { striatus }\end{array}$ & Ballochory & Tree / Shrub & Ballochory & 0.96 & Mean & Bossard 1991 cited in Zouhar (2005a) \\
\hline $\begin{array}{l}\text { Cytisus } \\
\text { scoparius, } C . \\
\text { striatus }\end{array}$ & Ballochory & Tree / Shrub & Ballochory & 1.12 & Median & Bossard 1991 cited in Zouhar (2005a) \\
\hline Dioscorea spp & Autochory & Forb & Gravity & 22 & Maximum & Beyerl 2001 cited in Gucker (2009c) \\
\hline Dioscorea spp & Autochory & Forb & Gravity & 10 & Mode & Beyerl 2001 cited in Gucker (2009c) \\
\hline $\begin{array}{l}\text { Dipsacus } \\
\text { fullonum }\end{array}$ & Agochory & Forb & Humans & 49 & Maximum & $\begin{array}{l}\text { Musser \& Parrish } 2002 \text { cited in Gucker } \\
\text { (2009d) }\end{array}$ \\
\hline $\begin{array}{l}\text { Dipsacus } \\
\text { fullonum }\end{array}$ & Autochory & Forb & Gravity & 1.5 & 99.9 Percentile & Werner 1975 cited in Gucker (2009d) \\
\hline $\begin{array}{l}\text { Dipsacus } \\
\text { fullonum }\end{array}$ & Autochory & Forb & Gravity & 4.5 & 98.3 Percentile & $\begin{array}{l}\text { Musser \& Parrish } 2002 \text { cited in Gucker } \\
\text { (2009d) }\end{array}$ \\
\hline $\begin{array}{l}\text { Eucalyptus } \\
\text { globulus }\end{array}$ & Pterometeorochory & Tree / Shrub & Wind & 20 & Mean & $\begin{array}{l}\text { Skolmen et al. } 1990 \text { cited in Esser } \\
\text { (1993a) }\end{array}$ \\
\hline Euphorbia esula & Ballochory & Forb & Ballochory & 4.5 & Maximum & Selleck 1958 cited in Gucker (2010a) \\
\hline Euphorbia esula & Ballochory & Forb & Ballochory & 4 & Maximum & $\begin{array}{l}\text { Hanson \& Rudd } 1933 \text { cited in Gucker } \\
(2010 a)\end{array}$ \\
\hline Euphorbia esula & Ballochory & Forb & Ballochory & 4.1 & Maximum & $\begin{array}{l}\text { Hanson \& Rudd } 1933 \text { cited in Gucker } \\
\text { (2010a) }\end{array}$ \\
\hline Euphorbia esula & Ballochory & Forb & Ballochory & 0.3 & 1 Percentile & $\begin{array}{l}\text { Hanson \& Rudd } 1933 \text { cited in Gucker } \\
(2010 a)\end{array}$ \\
\hline $\begin{array}{l}\text { Flourensia } \\
\text { cernua }\end{array}$ & Autochory & Tree / Shrub & Wind & 5 & Maximum & $\begin{array}{l}\text { Mauchamp et al. } 1993 \text { cited in Innes } \\
\text { (2010a) }\end{array}$ \\
\hline $\begin{array}{l}\text { Flourensia } \\
\text { cernua }\end{array}$ & Autochory & Tree / Shrub & Wind & 3 & Mode & $\begin{array}{l}\text { Mauchamp et al. } 1993 \text { cited in Innes } \\
\text { (2010a) }\end{array}$ \\
\hline
\end{tabular}


Table A4 (Continued):

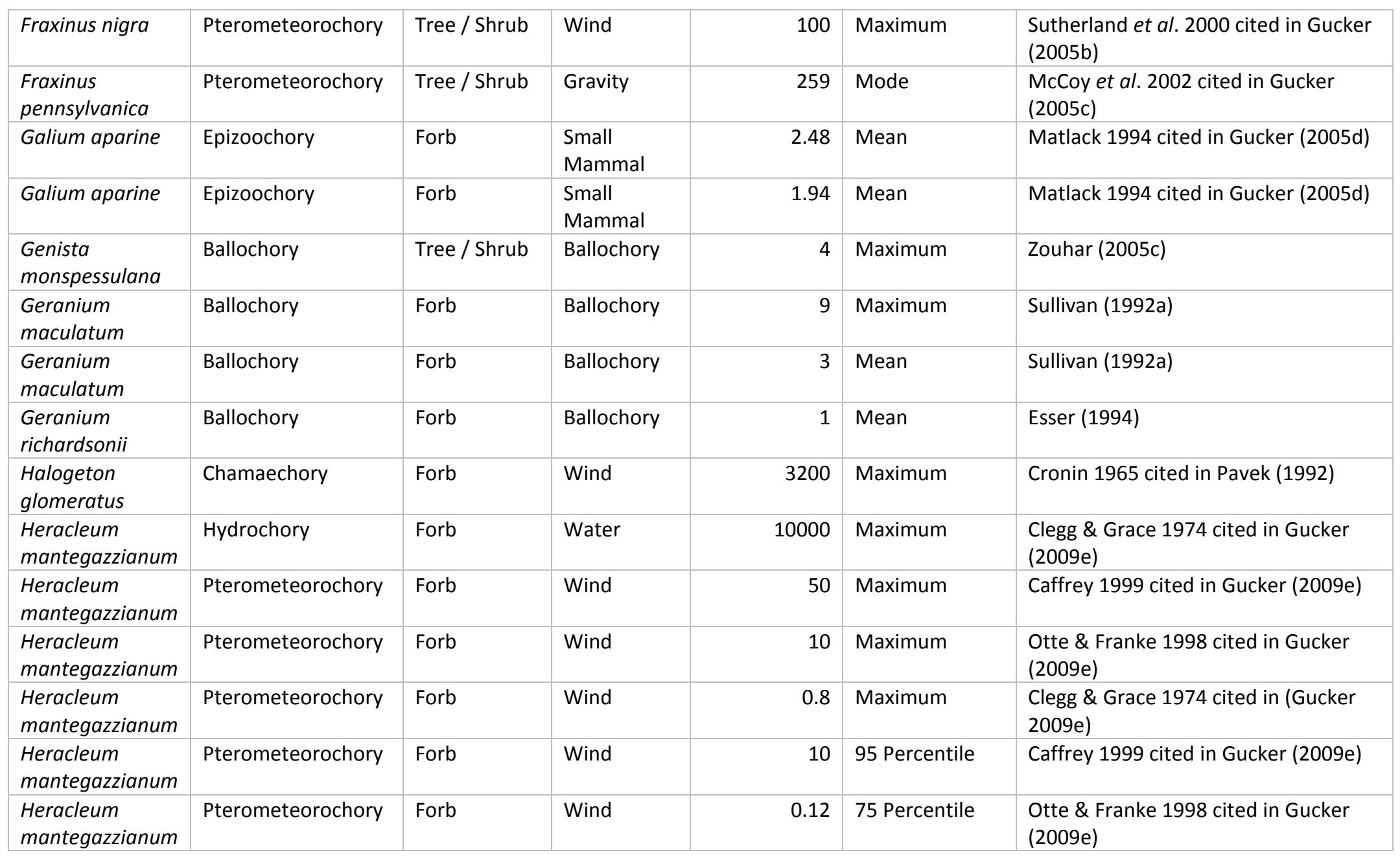


Table A4 (Continued):

\begin{tabular}{|c|c|c|c|c|c|c|}
\hline $\begin{array}{l}\text { Hieracium } \\
\text { aurantiacum }\end{array}$ & Trichometeorochory & Forb & Wind & 1 & 95 Percentile & Stergios 1976 cited in Stone (2010a) \\
\hline $\begin{array}{l}\text { Hieracium } \\
\text { aurantiacum }\end{array}$ & Trichometeorochory & Forb & Wind & 1 & 83 Percentile & Stergios 1976 cited in Stone (2010a) \\
\hline $\begin{array}{l}\text { Hypericum } \\
\text { perforatum }\end{array}$ & Autochory & Forb & Wind & 9 & 100 Percentile & $\begin{array}{l}\text { Tisdale et al } 1959 \text { cited in Zouhar } \\
\text { (2004) }\end{array}$ \\
\hline $\begin{array}{l}\text { Hypericum } \\
\text { perforatum }\end{array}$ & Autochory & Forb & Wind & 6 & 96 Percentile & $\begin{array}{l}\text { Tisdale et al } 1959 \text { cited in Zouhar } \\
\text { (2004) }\end{array}$ \\
\hline Isatis tinctoria & Autochory & Forb & Gravity & 2.4 & Maximum & $\begin{array}{l}\text { Farah 1987; Farah et al. } 1988 \text { cited in } \\
\text { Zouhar (2009) }\end{array}$ \\
\hline Isatis tinctoria & Autochory & Forb & Gravity & 0.54 & 95 Percentile & $\begin{array}{l}\text { Farah 1987; Farah et al. } 1988 \text { cited in } \\
\text { Zouhar (2009) }\end{array}$ \\
\hline $\begin{array}{l}\text { Juniperus } \\
\text { occidentalis }\end{array}$ & Autochory & Tree / Shrub & Gravity & 1.4 & Mean & Tirmenstein (1999b) \\
\hline Larix laricina & Pterometeorochory & Tree / Shrub & Gravity & 46 & Mode & $\begin{array}{l}\text { Brown et al. } 1988 \text { cited in Uchytil } \\
\text { (1991d) }\end{array}$ \\
\hline Larix occidentalis & Pterometeorochory & Tree / Shrub & Wind & 250 & Maximum & $\begin{array}{l}\text { Schmidt \& Shearer } 1995 \text { cited in Scher } \\
(2002)\end{array}$ \\
\hline Larix occidentalis & Pterometeorochory & Tree / Shrub & Wind & 100 & Mode & $\begin{array}{l}\text { Schmidt \& Shearer } 1995 \text { cited in Scher } \\
\text { (2002) }\end{array}$ \\
\hline Linaria spp & Pterometeorochory & Forb & Gravity & 1.5 & Maximum & $\begin{array}{l}\text { Nadeau \& King } 1991 \text { cited in Zouhar } \\
\text { (2003) }\end{array}$ \\
\hline Linaria spp & Pterometeorochory & Forb & Gravity & 0.5 & 80 Percentile & $\begin{array}{l}\text { Nadeau \& King } 1991 \text { cited in Zouhar } \\
\text { (2003) }\end{array}$ \\
\hline $\begin{array}{l}\text { Liquidambar } \\
\text { styraciflua }\end{array}$ & Pterometeorochory & Tree / Shrub & Wind & 183 & 100 Percentile & $\begin{array}{l}\text { Paton et al 1944; Kormanik } 1990 \text { cited } \\
\text { in Coladonato (1992) }\end{array}$ \\
\hline $\begin{array}{l}\text { Liquidambar } \\
\text { styraciflua }\end{array}$ & Pterometeorochory & Tree / Shrub & Wind & 61 & 96 Percentile & $\begin{array}{l}\text { Paton et al 1944; Kormanik } 1990 \text { cited } \\
\text { in Coladonato (1992) }\end{array}$ \\
\hline
\end{tabular}


Table A4 (Continued):

\begin{tabular}{|c|c|c|c|c|c|c|}
\hline $\begin{array}{l}\text { Liriodendron } \\
\text { tulipifera }\end{array}$ & Pterometeorochory & Tree / Shrub & Wind & 300 & Mode & Griffith (1991) \\
\hline $\begin{array}{l}\text { Lithocarpus } \\
\text { densiflorus }\end{array}$ & Autochory & Tree / Shrub & Gravity & 5 & Mode & Fryer (2008) \\
\hline Lupinus latifolius & Autochory & Forb & Gravity & 3 & Most & Dale 1989 cited in Reeves (2006) \\
\hline Lupinus perennis & Autochory & Forb & Gravity & 5 & Maximum & $\begin{array}{l}\text { Dirig 1994; Grigore \& Tramer } 1996 \\
\text { cited in Meyer (2006) }\end{array}$ \\
\hline Lythrum salicaria & Autochory & Forb & Gravity & 10 & Mode & $\begin{array}{l}\text { Thompson et al } 1987 \text { cited in Munger } \\
\text { (2002) }\end{array}$ \\
\hline $\begin{array}{l}\text { Melaleuca } \\
\text { quinquenervia }\end{array}$ & Autochory & Tree / Shrub & Gravity & 170 & Maximum & Woodall 1982 cited in Munger (2005) \\
\hline $\begin{array}{l}\text { Melaleuca } \\
\text { quinquenervia }\end{array}$ & Autochory & Tree / Shrub & Wind & 15 & Maximum & $\begin{array}{l}\text { Meskimen } 1962 \text { cited in Munger } \\
(2005)\end{array}$ \\
\hline $\begin{array}{l}\text { Paulownia } \\
\text { tomentosa }\end{array}$ & Pterometeorochory & Tree / Shrub & Wind & 3000 & Maximum & $\begin{array}{l}\text { Langdon \& Johnson 1994; Kuppinger } \\
2008 \text { cited in Innes (2009) }\end{array}$ \\
\hline Picea abies & Chamaechory & Tree / Shrub & Wind & 61 & Mode & Kostler 1956 cited in Sullivan (1994) \\
\hline $\begin{array}{l}\text { Picea } \\
\text { engelmannii }\end{array}$ & Pterometeorochory & Tree / Shrub & Wind & 91 & 90 Percentile & $\begin{array}{l}\text { Alexander \& Shepperd 1990; Nobel et } \\
\text { al. } 1978 \text { cited in Uchytil (1991e) }\end{array}$ \\
\hline Picea glauca & Pterometeorochory & Tree / Shrub & Wind & 400 & Maximum & $\begin{array}{l}\text { Benzie \& Blum 1989; Zasada } 1986 \\
\text { cited in Uchytil (1991e) }\end{array}$ \\
\hline Picea glauca & Pterometeorochory & Tree / Shrub & Wind & 64 & 90 Percentile & Zasada 1971 cited in Uchytil (1991e) \\
\hline Picea glauca & Pterometeorochory & Tree / Shrub & Wind & 91 & Mode & $\begin{array}{l}\text { Benzie \& Blum 1989; Zasada } 1986 \\
\text { cited in Uchytil (1991e) }\end{array}$ \\
\hline Picea glauca & Pterometeorochory & Tree / Shrub & Wind & 27 & 50 Percentile & Zasada 1971 cited in Uchytil (1991e) \\
\hline Picea mariana & Pterometeorochory & Tree / Shrub & Wind & 20 & Maximum & Johnston 1971 cited in Fryer 2014 \\
\hline Picea mariana & Pterometeorochory & Tree / Shrub & Wind & 60 & 100 Percentile & LeBarron 1933 cited in Fryer (2014) \\
\hline Picea mariana & Pterometeorochory & Tree / Shrub & Wind & 80 & Mode & $\begin{array}{l}\text { Tuskan \& Laughlin } 1991 \text { cited in Fryer } \\
\text { (2014) }\end{array}$ \\
\hline Picea mariana & Pterometeorochory & Tree / Shrub & Wind & 0 & 54 Percentile & LeBarron 1933 cited in Fryer (2014) \\
\hline
\end{tabular}


Table A4 (Continued):

\begin{tabular}{|c|c|c|c|c|c|c|}
\hline Picea mariana & Pterometeorochory & Tree / Shrub & Wind & 30 & 86 Percentile & LeBarron 1933 cited in Fryer (2014) \\
\hline Picea rubens & Autochory & Tree / Shrub & Wind & 61 & Maximum & $\begin{array}{l}\text { Govindaraju } 1988 \text { cited in Sullivan } \\
\text { (1993b) }\end{array}$ \\
\hline Pinus banksiana & Pterometeorochory & Tree / Shrub & Wind & 40 & Maximum & $\begin{array}{l}\text { McCaughey et al. } 1986 \text { cited in Carey } \\
\text { (1993a) }\end{array}$ \\
\hline $\begin{array}{l}\text { Pinus contorta } \\
\text { var. contorta }\end{array}$ & Autochory & Tree / Shrub & Gravity & 60 & Mean & Critchfield 1978 cited in Cope (1993) \\
\hline Pinus echinata & Pterometeorochory & Tree / Shrub & Gravity & 91 & Maximum & Lawson 1990 cited in Carey (1992a) \\
\hline Pinus jeffreyi & Dyszoochory & Tree / Shrub & $\begin{array}{l}\text { Small } \\
\text { Mammal }\end{array}$ & 63 & Maximum & $\begin{array}{l}\text { Vander Wall 1995; Vander Wall } 1993 \\
\text { cited in Gucker (2007a) }\end{array}$ \\
\hline Pinus jeffreyi & Dyszoochory & Tree / Shrub & $\begin{array}{l}\text { Small } \\
\text { Mammal }\end{array}$ & 25 & Mean & $\begin{array}{l}\text { Vander Wall } 1995 \text { cited in Gucker } \\
\text { (2007a) }\end{array}$ \\
\hline Pinus jeffreyi & Pterometeorochory & Tree / Shrub & Wind & 27 & Maximum & $\begin{array}{l}\text { Johnson et al. 2003; Lanner } 1999 \text { cited } \\
\text { in Gucker (2007a) }\end{array}$ \\
\hline $\begin{array}{l}\text { Pinus } \\
\text { monophylla }\end{array}$ & Dyszoochory & Tree / Shrub & Birds & 22000 & Maximum & $\begin{array}{l}\text { Lanner 1983; Lanner 1996; Tomback } \\
1994 \text { cited in Zouhar (2001) }\end{array}$ \\
\hline Pinus monticola & Pterometeorochory & Tree / Shrub & Wind & 800 & Maximum & Griffith (1992a) \\
\hline Pinus palustris & Pterometeorochory & Tree / Shrub & Wind & 20 & 71 Percentile & Boyer 1990 cited in Carey (1992b) \\
\hline $\begin{array}{l}\text { Pinus ponderosa } \\
\text { var arizonica }\end{array}$ & Pterometeorochory & Tree / Shrub & Wind & 37 & Maximum & $\begin{array}{l}\text { Gartner \& Thompson 1973; Howard } \\
2003\end{array}$ \\
\hline $\begin{array}{l}\text { Pinus ponderosa } \\
\text { var ponderosa }\end{array}$ & Pterometeorochory & Tree / Shrub & Wind & 31 & Maximum & Habeck (1992b) \\
\hline $\begin{array}{l}\text { Pinus ponderosa } \\
\text { var ponderosa }\end{array}$ & Pterometeorochory & Tree / Shrub & Wind & 161 & 92 Percentile & Habeck (1992b) \\
\hline $\begin{array}{l}\text { Pinus ponderosa } \\
\text { var ponderosa }\end{array}$ & Pterometeorochory & Tree / Shrub & Wind & 40 & 78 Percentile & Habeck (1992b) \\
\hline
\end{tabular}


Table A4 (Continued):

\begin{tabular}{|c|c|c|c|c|c|c|}
\hline $\begin{array}{l}\text { Pinus ponderosa } \\
\text { var scopulorum }\end{array}$ & Pterometeorochory & Tree / Shrub & Wind & 37 & Maximum & $\begin{array}{l}\text { Oliver \& Ryker } 1990 \text { cited in Howard } \\
(2003)\end{array}$ \\
\hline Pinus quadrifolia & Dyszoochory & Tree / Shrub & Birds & 20000 & Maximum & $\begin{array}{l}\text { Krugman \& Jenkinson } 1974 \text { cited in } \\
\text { Esser (1993b) }\end{array}$ \\
\hline Pinus resinosa & Pterometeorochory & Tree / Shrub & Wind & 12 & Mean & Rudolf 1990 cited in Hauser (2008) \\
\hline Pinus strobus & Pterometeorochory & Tree / Shrub & Wind & 60 & Mean & Carey $(1993 b)$ \\
\hline Pinus strobus & Pterometeorochory & Tree / Shrub & Wind & 210 & Mean & Carey (1993b) \\
\hline Pinus taeda & Pterometeorochory & Tree / Shrub & Wind & 91 & Maximum & Baker et al. 1990 cited in Carey (1990) \\
\hline Pinus virginiana & Pterometeorochory & Tree / Shrub & Wind & 30 & Mode & $\begin{array}{l}\text { Carter \& Snow } 1990 \text { cited in Sullivan } \\
\text { (1993d) }\end{array}$ \\
\hline $\begin{array}{l}\text { Polygonum } \\
\text { sachalinese }\end{array}$ & Pterometeorochory & Forb & Gravity & 16 & Maximum & $\begin{array}{l}\text { Tiebre et al } 2007 \text { cited in (Stone } \\
\text { 2010b) }\end{array}$ \\
\hline $\begin{array}{l}\text { Populus alba } \\
\text { hybrids }\end{array}$ & Trichometeorochory & Tree / Shrub & Wind & 30000 & Maximum & $\begin{array}{l}\text { van der Pijl } 1972 \text { cited in Gucker } \\
\text { (2010b) }\end{array}$ \\
\hline $\begin{array}{l}\text { Populus } \\
\text { balsamifera }\end{array}$ & Trichometeorochory & Tree / Shrub & Wind & 200 & Mode & $\begin{array}{l}\text { Haeussler \& Coates } 1986 \text { cited in } \\
\text { Harris (1990) }\end{array}$ \\
\hline $\begin{array}{l}\text { Populus } \\
\text { tremuloides }\end{array}$ & Trichometeorochory & Tree / Shrub & Wind & 500 & Mode & Howard 1996 \\
\hline Prosopis velutina & Dyszoochory & Tree / Shrub & $\begin{array}{l}\text { Small } \\
\text { Mammal }\end{array}$ & 30 & Mode & $\begin{array}{l}\text { Reynolds 1954; Glendening \& Paulsen } \\
1955 \text { cited in Uchytil (1990) }\end{array}$ \\
\hline $\begin{array}{l}\text { Pseudotsuga } \\
\text { macrocarpa }\end{array}$ & Autochory & Tree / Shrub & Gravity & 3 & Mode & $\begin{array}{l}\text { McDonald } 1990 \text { cited in Howard } \\
\text { (1992a) }\end{array}$ \\
\hline $\begin{array}{l}\text { Pseudotsuga } \\
\text { menziesii var } \\
\text { glauca }\end{array}$ & Pterometeorochory & Tree / Shrub & Wind & 244 & Maximum & Shearer 1981; Steinberg 2002 \\
\hline
\end{tabular}


Table A4 (Continued):

\begin{tabular}{|c|c|c|c|c|c|c|}
\hline $\begin{array}{l}\text { Pseudotsuga } \\
\text { menziesii var } \\
\text { glauca }\end{array}$ & Pterometeorochory & Tree / Shrub & Wind & 2000 & Maximum & $\begin{array}{l}\text { Burns \& Honkala } 1990 \text { cited in } \\
\text { Steinberg (2002) }\end{array}$ \\
\hline $\begin{array}{l}\text { Pseudotsuga } \\
\text { menziesii var } \\
\text { glauca }\end{array}$ & Pterometeorochory & Tree / Shrub & Wind & 80 & 97 Percentile & $\begin{array}{l}\text { Hermann \& Lavender } 1990 \text { cited in } \\
\text { Steinberg (2002) }\end{array}$ \\
\hline $\begin{array}{l}\text { Pseudotsuga } \\
\text { menziesii var } \\
\text { glauca }\end{array}$ & Pterometeorochory & Tree / Shrub & Wind & 100 & Mode & $\begin{array}{l}\text { Burns \& Honkala } 1990 \text { cited in } \\
\text { Steinberg (2002) }\end{array}$ \\
\hline Quercus agrifolia & Dyszoochory & Tree / Shrub & Birds & 12500 & Maximum & Steinberg (2002) \\
\hline $\begin{array}{l}\text { Quercus } \\
\text { garryana }\end{array}$ & Chamaechory & Tree / Shrub & Gravity & 6.65 & Maximum & Voeks 1981 cited in Gucker (2007b) \\
\hline $\begin{array}{l}\text { Quercus } \\
\text { garryana }\end{array}$ & Dyszoochory & Tree / Shrub & $\begin{array}{l}\text { Small } \\
\text { Mammal }\end{array}$ & 8 & Mean & Voeks 1981 cited in Gucker (2007b) \\
\hline $\begin{array}{l}\text { Quercus } \\
\text { garryana }\end{array}$ & Dyszoochory & Tree / Shrub & Birds & 50 & Mean & Voeks 1981 cited in Gucker (2007b) \\
\hline $\begin{array}{l}\text { Quercus } \\
\text { macrocarpa }\end{array}$ & Dyszoochory & Tree / Shrub & $\begin{array}{l}\text { Small } \\
\text { Mammal }\end{array}$ & 150 & Maximum & $\begin{array}{l}\text { Hewitt \& Kellman } 2002 \text { cited in } \\
\text { Gucker (2011) }\end{array}$ \\
\hline $\begin{array}{l}\text { Quercus } \\
\text { macrocarpa }\end{array}$ & Dyszoochory & Tree / Shrub & $\begin{array}{l}\text { Small } \\
\text { Mammal }\end{array}$ & 51.5 & Maximum & Stapanian 1984 cited in Gucker (2011) \\
\hline $\begin{array}{l}\text { Quercus } \\
\text { macrocarpa }\end{array}$ & Dyszoochory & Tree / Shrub & $\begin{array}{l}\text { Small } \\
\text { Mammal }\end{array}$ & 18 & Mean & Stapanian 1984 cited in Gucker (2011) \\
\hline Quercus palustris & Dyszoochory & Tree / Shrub & Birds & 1900 & Maximum & $\begin{array}{l}\text { Darley-Hill \& Johnson } 1981 \text { cited in } \\
\text { Carey (1992c) }\end{array}$ \\
\hline Quercus palustris & Dyszoochory & Tree / Shrub & Birds & 1100 & Mean & $\begin{array}{l}\text { Darley-Hill \& Johnson } 1981 \text { cited in } \\
\text { Carey (1992c) }\end{array}$ \\
\hline Quercus rubra & Dyszoochory & Tree / Shrub & Birds & 5000 & Maximum & $\begin{array}{l}\text { Knapp \& Rice 1998; Kitteredge \& } \\
\text { Ashton } 1990 \text { cited in Tirmenstein } \\
\text { (1991) }\end{array}$ \\
\hline
\end{tabular}


Table A4 (Continued):

\begin{tabular}{|c|c|c|c|c|c|c|}
\hline Quercus rubra & Dyszoochory & Tree / Shrub & $\begin{array}{l}\text { Small } \\
\text { Mammal }\end{array}$ & 30 & Mode & $\begin{array}{l}\text { Crow } 1988 \text { cited in Tirmenstein } \\
\text { (1991b) }\end{array}$ \\
\hline Salix discolor & Trichometeorochory & Tree / Shrub & Wind & 287 & Mean & $\begin{array}{l}\text { Robinson \& Handel } 1993 \text { cited in } \\
\text { (Gucker 2007c) }\end{array}$ \\
\hline $\begin{array}{l}\text { Sarcobatus } \\
\text { vermiculatus }\end{array}$ & Pterometeorochory & Tree / Shrub & Wind & 700 & Maximum & $\begin{array}{l}\text { Fort \& Richards } 1998 \text { cited in } \\
\text { Anderson (2004) }\end{array}$ \\
\hline $\begin{array}{l}\text { Sequoia } \\
\text { sempervirens }\end{array}$ & Pterometeorochory & Tree / Shrub & Wind & 122 & Mean & $\begin{array}{l}\text { Olson et al. } 1990 \text { cited in Griffith } \\
\text { (1992b) }\end{array}$ \\
\hline $\begin{array}{l}\text { Solidago } \\
\text { canadensis }\end{array}$ & Trichometeorochory & Forb & Wind & 2 & Mode & $\begin{array}{l}\text { Werner } 1980 \text { cited in Coladonato } \\
\text { (1993) }\end{array}$ \\
\hline Sonchus arvensis & Trichometeorochory & Forb & Wind & 100 & Maximum & $\begin{array}{l}\text { Hume and Archibold } 1986 \text { cited in } \\
\text { McWilliams (2004) }\end{array}$ \\
\hline Sonchus arvensis & Trichometeorochory & Forb & Wind & 3.34 & Mean & $\begin{array}{l}\text { Sheldon \& Burrows } 1973 \text { cited in } \\
\text { McWilliams (2004) }\end{array}$ \\
\hline Sonchus arvensis & Trichometeorochory & Forb & Wind & 10 & Mean & $\begin{array}{l}\text { Sheldon \& Burrows } 1973 \text { cited in } \\
\text { McWilliams (2004) }\end{array}$ \\
\hline $\begin{array}{l}\text { Sorbus } \\
\text { americana }\end{array}$ & Endozoochory & Tree / Shrub & Birds & 4830 & Maximum & Stiles 1980 cited in Sullivan (1992b) \\
\hline $\begin{array}{l}\text { Thuja } \\
\text { occidentalis }\end{array}$ & Pterometeorochory & Tree / Shrub & Wind & 60 & Mode & $\begin{array}{l}\text { Curtis 1946; Curtis 1959; Johnston } \\
1990 \text { cited in Carey (1993c) }\end{array}$ \\
\hline Thuja plicata & Pterometeorochory & Tree / Shrub & Wind & 122 & Maximum & $\begin{array}{l}\text { Arno 1977; MacCaughey et al. 1986; } \\
\text { Minroe } 1990 \text { cited in Tesky (1992a) }\end{array}$ \\
\hline $\begin{array}{l}\text { Tragopogon } \\
\text { dubius }\end{array}$ & Trichometeorochory & Forb & Wind & 250 & Maximum & Gross 1980 cited in Gucker (2008a) \\
\hline $\begin{array}{l}\text { Tragopogon } \\
\text { dubius }\end{array}$ & Trichometeorochory & Forb & Wind & 5 & 90 Percentile & Gross 1980 cited in Gucker (2008a) \\
\hline
\end{tabular}


Table A4 (Continued):

\begin{tabular}{|c|c|c|c|c|c|c|}
\hline $\begin{array}{l}\text { Tsuga } \\
\text { canadensis }\end{array}$ & Pterometeorochory & Tree / Shrub & Gravity & 21 & Mode & $\begin{array}{l}\text { Godman \& Lancaster } 1990 \text { cited in } \\
\text { Carey }(1993 d)\end{array}$ \\
\hline $\begin{array}{l}\text { Tsuga } \\
\text { heterophylla }\end{array}$ & Pterometeorochory & Tree / Shrub & Wind & 1150 & Maximum & $\begin{array}{l}\text { Owens \& Molder } 1984 \text { cited in Tesky } \\
\text { (1992b) }\end{array}$ \\
\hline Tussilago farfara & Trichometeorochory & Forb & Wind & 600 & Maximum & Bakker 1960 cited in Innes (2011) \\
\hline Tussilago farfara & Trichometeorochory & Forb & Wind & 100 & Mode & Bakker 1960 cited in Innes (2011) \\
\hline Ulex europaeus & Autochory & Tree / Shrub & Gravity & 0 & 39.4 Percentile & Hill et al. 1996 cited in Zouhar (2005d) \\
\hline Ulex europaeus & Autochory & Tree / Shrub & Gravity & 1 & 55.7 Percentile & Hill et al. 1996 cited in Zouhar (2005d) \\
\hline Ulex europaeus & Ballochory & Tree / Shrub & Ballochory & 5 & Maximum & $\begin{array}{l}\text { Moss } 1959 \text { cited by Richardson \& Hill } \\
1998 \text { cited in Zouhar }(2005 d)\end{array}$ \\
\hline $\begin{array}{l}\text { Ulmus } \\
\text { americana }\end{array}$ & Pterometeorochory & Tree / Shrub & Wind & 400 & Maximum & Coladonato (1992b) \\
\hline $\begin{array}{l}\text { Ulmus } \\
\text { americana }\end{array}$ & Pterometeorochory & Tree / Shrub & Wind & 90 & Mode & Coladonato (1992b) \\
\hline $\begin{array}{l}\text { Verbascum } \\
\text { thapsus }\end{array}$ & Autochory & Forb & Gravity & 11 & Maximum & $\begin{array}{l}\text { Gross \& Werner 1978; Gross } 1980 \\
\text { cited in Gucker (2008b) }\end{array}$ \\
\hline $\begin{array}{l}\text { Verbascum } \\
\text { thapsus }\end{array}$ & Autochory & Forb & Gravity & 1 & Median & $\begin{array}{l}\text { Gross \& Werner 1978; Gross } 1980 \\
\text { cited in Gucker (2008b) }\end{array}$ \\
\hline $\begin{array}{l}\text { Washingtonia } \\
\text { filifera }\end{array}$ & Endozoochory & Tree / Shrub & $\begin{array}{l}\text { Large } \\
\text { Mammal }\end{array}$ & 11300 & Maximum & $\begin{array}{l}\text { Gese et al } 1988 \text { cited in Howard } \\
\text { (1992b) }\end{array}$ \\
\hline Yucca Brevifolia & Dyszoochory & Tree / Shrub & $\begin{array}{l}\text { Small } \\
\text { Mammal }\end{array}$ & 251 & Maximum & Lenz 2001 cited in Gucker (\$2006c) \\
\hline
\end{tabular}




\section{APPENDIX 5: OVERVIEW, DESIGN CONCEPTS AND DETAILS (ODD)}

\section{ODD Purpose}

The purpose of the model is to simulate fine-scale regular movements of oilbirds based on the underlying environment and memory, and investigate how the interactions between environmentally driven movements and the presence of five key food sources influence the sustainability of an oilbird population for $2500 \mathrm{~km}^{2}$ grids in Venezuela.

\section{ODD State Variables and scales}

All individual oilbirds are treated identically, with no low level state variables. Oilbirds have variable energy, which is a combination between energy obtained from the fruit and energy utilized to move. The environment is characterized by three states: land cover, resource availability, and memory. Land cover is a static variable, as it assumed it will not change substantially when the model is run for one year. Land cover is comprised of four covers: evergreen broadleaf forest, cropland, other habitat and distance to rivers. The land cover information was obtained from MODIS (MCD12Q1) land cover product (LP DAAC 2010) and river data from HydroSHEDS (Lehner et al. 2008). Resource availability is defined the by annual amount of fruit produced by the oilbirds food sources. Fruit production is therefore the annual amount of fruit produced at a $1 \mathrm{~km}$ resolution for five widespread tree species which make up the oilbirds diet (Bosque et al. 1995). An estimate of 250 fruits per tree for Nectandra membranacea, Ocotea floribunda, Persea caerulea and Prestoea acuminata, and of 1250 fruits per tree for Euterpe precatoria was made for species. Tree density, which was taken as the maximum abundance recorded in a comprehensive study of the Amazonian tree flora (ter Steege et al. 2013), was then used to extrapolate the total amount of fruit to a $1 \mathrm{~km}$ resolution. 
Memory is recorded as a binary variable, meaning if a grid is visited by an individual oilbird, the individual remembers the coordinates of the location.

\section{ODD Process Overview and Scheduling}

The model is run at 10 minute time steps between the hours of $10 \mathrm{pm}$ and $10 \mathrm{am}$ for a one-year period. The probability of movement by an individual was developed by using step-selection function (SSF). Five possible steps are identified by an individual oilbird, and the variables along the steps are analyzed using the model generated from the earlier conditional logistic regression parameters. From this regression model, one final step is chosen based on the likelihood of each of the possible steps available.

\section{ODD Design Concepts}

Emergence: The emergent phenomenon from the interactions between the agents and the environment is a measure of how sustainable the environment is for the agents. As such, if the agents do not consume a suitable amount of resources then they die, and if they do consume a suitable amount of resources they do not die. A starting value of energy was equated to an equivalent of 1 weekly estimate of food intake (consumption of 1 biotic resource at each time step $=540$ ) due to the relatively high energy demands of frugivores. The final outcome of this model is a figure representing the number of agents remaining after the model has simulated movement and environmental interactions for a year.

Adaptation: The agents adapt their memory of the environment by storing the locations of previously visited patches. The agents are allowed to move through the landscape for a time equivalent to a month before the agents interacts with the

environment and the subsequent processes of resource depletion and death are allowed to 
operate. This temporal lag builds up the memory of individual agents such that they do not succumb to death straight away based on the stochasticity of where they were 'born'.

Interaction: Two types of interactions occur between the agent and the environment. The first interaction is whereby the environment influences the agent's movements, and is underlined by the SSF model. The second interaction is where the agent's movement influences the environment. If resource availability for the patch of environment the agent has moved into is greater than zero, then the fruit level will deplete for every time step it is occupied. Based on maximum values found in seed traps from Bosque et al. (1995) and the size of the fruit, it has been specified in the model that the agents can consume up to 20 pieces during one 10 minute time period (fruit consumption can be considered equivalent to energy). Each movement step can utilize up to 10 pieces of energy.

Stochasticity: All step lengths and turn angles are derived from empirical distributions obtained from the telemetry data. Equal interval frequencies of $20^{\circ}$ bins were used to draw the turn angle value, with intervals of $100 \mathrm{~m}$ for values between $0 \mathrm{~m}$ and $1,000 \mathrm{~m}$, and intervals of $1,000 \mathrm{~m}$ for values between $1,000 \mathrm{~m}$ and $10,000 \mathrm{~m}$ used to draw the step length. By using uneven interval frequencies, the possibility that unbalanced step lengths are selected is controlled, as these have been found to inflate coefficient values when used in SSF (Holloway \& Miller 2014). Coefficients from the case-control regression model were used to model the likelihood that a step would be selected. The amount of energy gained from food consumption is potentially double that used of the energy used to move. Preliminary investigation of spatial simulation found that specifying equal energy gain and loss resulted in the death of all individuals almost immediately. This is obviously a subjective decision that could influence the results, which should be acknowledged, but a variant of a common assumption agent-based 187 
modelling (Wilensky 1997). To incorporate some stochasticity into this decision, these values were generated randomly up to the specified values, negating some of this potential bias.

\section{ODD Initialization}

Each simulation started with 1,000 oilbirds randomly placed in the study area and ran for a year. This population value was estimated based on the $90^{\text {th }}$ percentile of observed oilbird populations in Venezuela (Herrera 2003). Land cover and vegetation presence was imported using the import.raster function. Empirical distributions of the turn angle and step length were imported as text files using the import function.

\section{ODD Submodels}

\section{Step-Selection Function}

$$
\widehat{w}(x)=\exp \left(\beta_{1} x_{1}+\beta_{2} x_{2}+\ldots+\beta_{n} x_{n}\right)
$$

where $\beta_{n}$ is the coefficient estimated by the conditional logistic regression for the variable $x_{n}$. The final model used is in Table A.5.1.

Table A5: The results of the conditional logistic regression models used for stepselection function. ${ }^{*}$ significant at $\alpha<0.1, * *$ significant at $\alpha<0.05$.

\begin{tabular}{l|c|} 
Variables & \multicolumn{1}{c}{$\begin{array}{l}\text { Coefficient } \\
\text { Error) }\end{array}$} \\
\hline Evergreen Forest & $0.90630(0.46580)^{*}$ \\
\hline Cropland & $0.79150(0.54720)$ \\
\hline Rivers & $-0.00020(0.00004)^{* *}$ \\
\hline Memory & $-3.11500(0.97270)^{* *}$ \\
\hline Resources & $-1.34000(0.50440)^{* *}$ \\
\hline Memory*Resources & $3.39300(0.98660)^{* *}$ \\
\cline { 2 - 2 }
\end{tabular}




\section{Likelihood}

$$
L=\widehat{w}(x) /(1+\widehat{w}(x))
$$

\section{Death}

If an individual oilbirds energy drops below zero, then the oilbird dies. 
Appendix 6: NetLogo Code

\section{NetLogo World}
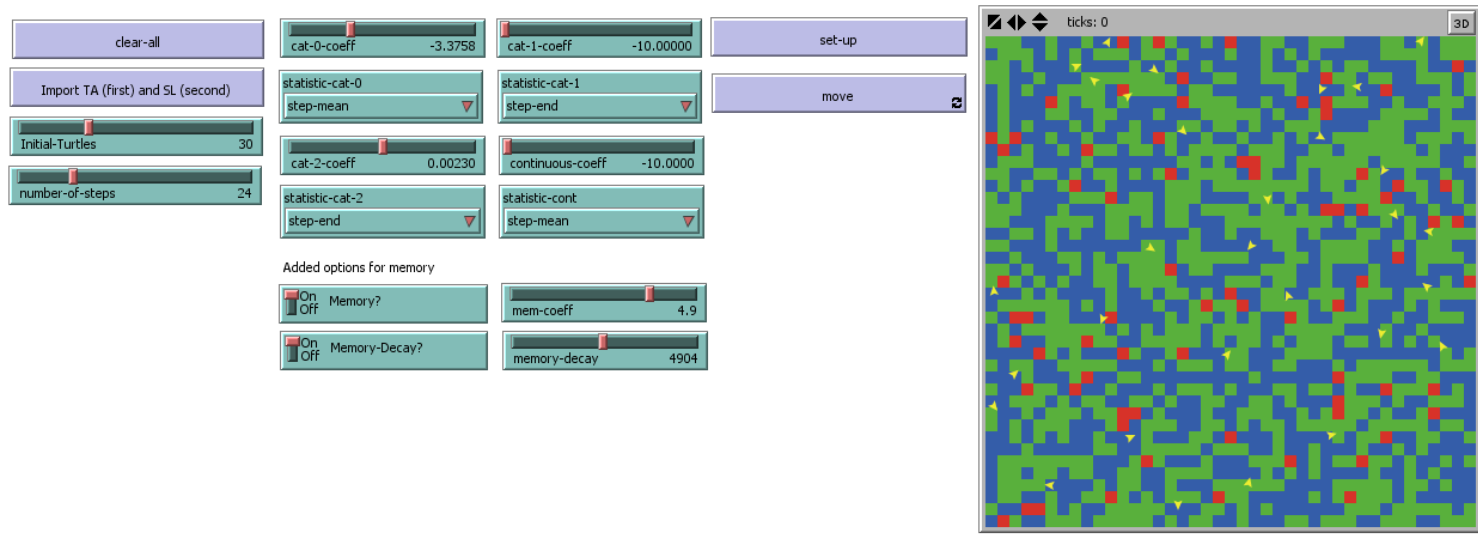

Figure A6: Screenshot of NetLogo World

\section{NetLogo Code}

Please see electronic Appendix 6. 


\section{References}

Abecasis, D., Afonso, P., \& Erzini, K. (2014). Combining multispecies home range and distribution models aids assessment of MPA effectiveness. Mar Ecol Prog Ser, 513, 155-169.

Adams, V.M., Petty, A.M., Douglas, M.M., Buckley, Y.M., Ferdinands, K.B., Okazaki, T., Ko., D.W., \& Setterfield, S.A. (2015). Distribution, demography and dispersal model of spatial spread of invasive plant populations with limited data. Methods in Ecology and Evolution, 6(7), 782-794.

Aguilar, M., \& Lado, C. (2012). Ecological niche models reveal the importance of climate variability for the biogeography of protosteloid amoebae. The ISME journal, 6(8), 1506-1514.

Aguilar, M., Fiore-Donno, A. M., Lado, C., \& Cavalier-Smith, T. (2013). Using environmental niche models to test the 'everything is everywhere' hypothesis for Badhamia. ISME J, 8, 737-45.

Ahearn, S.C., Smith, J.L.D., Joshi, A.R., \& Ding, J. (2001). TIGMOD: an individualbased spatially explicit model for simulating tiger/human interaction in multiple use forests. Ecological Modelling, 140(1), 81-97.

Ai, D., Desjardins-Proulx, P., Chu, C., \& Wang, G. (2012). Immigration, local dispersal limitation, and the repeatability of community composition under neutral and niche dynamics. PloS one, 7(9), e46164.

Alagador, D., Cerdeira, J.O., \& Araújo, M.B. (2014). Shifting protected areas: scheduling spatial priorities under climate change. Journal of applied ecology, 51(3), 703713.

Alford, R. A., Bradfield, K.S., \& Richards, S.J. (2007). Ecology: Global warming and amphibian losses. Nature, 447(7144), E3-E4.

Anderson, M.D. (2004). Sarcobatus vermiculatus. In: Fire Effects Information System, [Online]. U.S. Department of Agriculture, Forest Service, Rocky Mountain Research Station, Fire Sciences Laboratory (Producer). Available: http://www.fs.fed.us/database/feis/ [].

Anderson, R.P. (2013). A framework for using niche models to estimate impacts of climate change on species distributions. Annals of the New York Academy of Sciences, 1297(1), 8-28.

Araújo, M.B., Cabeza, M., Thuiller, W., Hannah, L., \& Williams, P.H. (2004). Would climate change drive species out of reserves? An assessment of existing reserveselection methods. Global Change Biology, 10 (9), 1618-1626. 
Araújo, M.B. \& Luoto, M. (2007). The importance of biotic interactions for modelling species distributions under climate change. Global Ecology and Biogeography, 16(6), 743-753.

Araújo, M.B., \& New, M. (2007). Ensemble forecasting of species distributions. Trends in ecology and evolution, 22(1), 42-47.

Araújo, M.B., Pearson, R.G., Thuiller, W., \& Erhard, M. (2005). Validation of speciesclimate impact models under climate change. Global Change Biology 11, 1-10.

Araújo, M.B., Thuiller, W., \& Pearson, R.G. (2006). Climate warming and the decline of amphibians and reptiles in Europe. Journal of Biogeography, 33 (10), 1712-1728.

Arthur, S.M., Manly, B.F.J., McDonald, L.L. \& Garner, G.W. (1996). Assessing habitat selection when availability changes. Ecology, 77(1), 215-227.

Ashcroft, M.B., \& Major, R.E. (2013). Importance of matrix permeability and quantity of core habitat for persistence of a threatened saltmarsh bird. Austral Ecology, 38(3), 326-337.

Baillie, S.R., Marchant, J.H., Leech, D.I., Massimino, D., Sullivan, M.J.P., Eglington, S.M., Barimore, C., Dadam, D., Downie, I.S., Harris, S.J., Kew, A.J., Newson, S.E., Noble, D.G., Risely, K., \& Robinson, R.A., (2014). BirdTrends 2014: trends in numbers, breeding success and survival for UK breeding birds [online]. BTO Research Report 662. BTO, Thetford. Available from http://www.bto.org/birdtrends.

Balmer, D.E., Gillings, S., Caffrey, B.J., Swann, R.L., Downie, I.S., \& Fuller R.J., (2013). Bird Atlas 2007-11: the Breeding and Wintering Birds of Britain and Ireland. BTO, Thetford.

Barbet-Massin, M., Jiguet, F., Albert, C.H., \& Thuiller, W. (2012). Selecting pseudoabsences for species distribution models: how, where and how many?. Methods in Ecology and Evolution, 3(2), 327-338.

Barve, N., Barve, V., Jiménez-Valverde, A., Lira-Noriega, A., Maher, S.P., Peterson, A.T., Soberón, J., \& Villalobos, F. (2011). The crucial role of the accessible area in ecological niche modeling and species distribution modeling. Ecological Modelling, 222(11), 1810-1819.

Bateman, B.L., Murphy, H.T., Reside, A.E., Mokany, K., \& VanDerWal, J. (2013). Appropriateness of full-, partial-and no-dispersal scenarios in climate change impact modelling. Diversity and Distributions, 19(10), 1224-1234.

Beauchesne, D., Jaeger, J.A., \& St-Laurent, M.H. (2013). Disentangling woodland caribou movements in response to clearcuts and roads across temporal scales. PloS one, 8(11), e77514. 
Belaire, J., Kreakie, B.J., Keitt, T., \& Minor, E. (2014). Predicting and mapping potential whooping crane stopover habitat to guide site selection for wind energy projects. Conservation biology, 28(2), 541-550.

Bendiksby, M., Mazzoni, S., Jørgensen, M.H., Halvorsen, R., \& Holien, H. (2014). Combining genetic analyses of archived specimens with distribution modelling to explain the anomalous distribution of the rare lichen Staurolemma omphalarioides: long-distance dispersal or vicariance?.Journal of Biogeography, 41(11), 2020-2031.

Benhamou, S. (2007). How many animals really do the Levy walk?. Ecology, 88(8), 1962-1969.

Benhamou, S. (2014). Of scales and stationarity in animal movements. Ecology letters, 17(3), 261-272.

Beschta, R.L., \& Ripple, W.J. (2006). River channel dynamics following extirpation of wolves in northwestern Yellowstone National Park, USA. Earth Surface Processes and Landforms, 31(12), 1525-1539.

Betts, M.G., Fahrig, L., Hadley, A.S., Halstead, K.E., Bowman, J., Robinson, W.D., Wiens, J.A., \& Lindenmayer, D.B. (2014). A species-centered approach for uncovering generalities in organism responses to habitat loss and fragmentation. Ecography, 37(6), 517-527.

Beyer, H.L. (2012). Geospatial Modelling Environment. Available at: http://www.spatialecology.com/gme/gmehelp.htm, [Accessed 12/12/13].

Bjørneraas, K., Solberg, E.J., Herfindal, I., Moorter, B.V., Rolandsen, C.M., Tremblay, J.P., Skarpe, C., Sæther, B.E., Eriksen, R., \& Astrup, R. (2011). Moose Alces alces habitat use at multiple temporal scales in a human-altered landscape. Wildlife Biology, 17(1), 44-54.

Blaustein, A.R., Han, B.A., Relyea, R.A., Johnson, P.T., Buck, J.C., Gervasi, S.S., \& Kats, L.B. (2011). The complexity of amphibian population declines: understanding the role of cofactors in driving amphibian losses. Annals of the New York Academy of Sciences, 1223(1), 108-119.

Blois, J.L., Williams, J.W., Fitzpatrick, M.C., Ferrier, S., Veloz, S.D., He, F., Liu, Z., Manion, G. \& Otto-Bliesner, B. (2013). Modeling the climatic drivers of spatial patterns in vegetation composition since the Last Glacial Maximum. Ecography, 36(4), 460-473.

Bolker, B.M., Brooks, M.E., Clark, C.J., Geange, S.W., Poulsen, J.R., Stevens, M.H.H., \& White, J.S.S. (2009). Generalized linear mixed models: a practical guide for ecology and evolution. Trends in Ecology and Evolution, 24 (3), 127-135.

Bosque, C., Ramírez, R., \& Rodríguez, D. (1995). The diet of the Oilbird in Venezuela. Ornitologia Neotropical, 6, 67-80. 
Boyce, M.S., Pitt, J., Northrup, J.M., Morehouse, A.T., Knopff, K.H., Cristescu, B., \& Stenhouse, G.B. (2010). Temporal autocorrelation functions for movement rates from global positioning system radiotelemetry data.Philosophical Transactions of the Royal Society of London B: Biological Sciences, 365(1550), 2213-2219.

Boyce, M.S., Vernier, P.R., Nielsen, S.E. \& Schmiegelow, F.K. (2002). Evaluating resource selection functions. Ecological modelling, 157(2), 281-300.

Boyer, D., Ramos-Fernández, G., Miramontes, G., Mateos, O., Cocho, J.L., Larralde, H., Ramos, H., \& Rojas, F. (2006). Scale-free foraging by primates emerges from their interaction with a complex environment. Proceedings of the Royal Society of London. Series B 273, 1743-1750.

Bradley, B.A., Early, R., \& Sorte, C.J. (2015). Space to invade? Comparative range infilling and potential range of invasive and native plants. Global Ecology and Biogeography, 24(3), 348-359.

Brambilla, M., \& Saporetti, F. (2014). Modelling distribution of habitats required for different uses by the same species: Implications for conservation at the regional scale. Biological Conservation, 174, 39-46.

Broenniman, O., Petitpierre, B., Randin, C., Engler, R., Breiner, F., D’Amen, M., Pellissier, L., Pottier, J., Pio, D., Garcia Mateo, R., Di Cola, V., Hordijk, W., Dubuis, A., Scherrer, D., Salamin, N., \& Guisan, A. (2014). ecospat: Spatial ecology miscellaneous methods. R package version 1.0.

Bush, A.A., Nipperess, D.A., Duursma, D.E., Theischinger, G., Turak, E., \& Hughes, L. (2014). Continental-scale assessment of risk to the Australian Odonata from climate change. PloS one, 9(2), e88958.

Carey, J.H. (1992a). Pinus echinata. In: Fire Effects Information System, [Online]. U.S. Department of Agriculture, Forest Service, Rocky Mountain Research Station, Fire Sciences Laboratory (Producer). Available: http://www.fs.fed.us/database/feis/ [2016, March 29].

Carey, J.H. (1992b). Pinus palustris. In: Fire Effects Information System, [Online]. U.S. Department of Agriculture, Forest Service, Rocky Mountain Research Station, Fire Sciences Laboratory (Producer). Available: http://www.fs.fed.us/database/feis/ [2016, March 29].

Carey, J.H. (1992c). Quercus palustris. In: Fire Effects Information System, [Online]. U.S. Department of Agriculture, Forest Service, Rocky Mountain Research Station, Fire Sciences Laboratory (Producer). Available: http://www.fs.fed.us/database/feis/ [2016, March 29].

Carey, J.H. (1992d). Pinus taeda. In: Fire Effects Information System, [Online]. U.S. Department of Agriculture, Forest Service, Rocky Mountain Research Station, Fire Sciences Laboratory (Producer). Available: http://www.fs.fed.us/database/feis/ [2016, March 29]. 
Carey, J.H. (1993a). Pinus banksiana. In: Fire Effects Information System, [Online]. U.S. Department of Agriculture, Forest Service, Rocky Mountain Research Station, Fire Sciences Laboratory (Producer). Available: http://www.fs.fed.us/database/feis/ [2016, March 29].

Carey, J.H. (1993b). Pinus strobus. In: Fire Effects Information System, [Online]. U.S. Department of Agriculture, Forest Service, Rocky Mountain Research Station, Fire Sciences Laboratory (Producer). Available: http://www.fs.fed.us/database/feis/ [2016, March 29].

Carey, J.H. (1993c). Thuja occidentalis. In: Fire Effects Information System, [Online]. U.S. Department of Agriculture, Forest Service, Rocky Mountain Research Station, Fire Sciences Laboratory (Producer). Available: http://www.fs.fed.us/database/feis/ [2016, March 29].

Carey, J.H. (1993d). Tsuga canadensis. In: Fire Effects Information System, [Online]. U.S. Department of Agriculture, Forest Service, Rocky Mountain Research Station, Fire Sciences Laboratory (Producer). Available: http://www.fs.fed.us/database/feis/ [2016, March 29].

Carey, P.D., Wallis, S., Chamberlain, P.M., Cooper, A., Emmett, B.A., Maskell, L.C., McCann, T., Murphy, J., Norton, L.R., Reynolds, B., Scott, W.A., Simpson, I.C., Smart, S.M., \& Ullyett, J.M., (2008). Countryside Survey: UK Results from 2007. Wallingford: NERC/Centre for Ecology and Hydrology.

Carstens B.C., \& Richards, C. (2007) Integrating coalescent and ecological niche modeling in comparative phylogeography. Evolution 61, 1439-1454.

Center for International Earth Science Information Network - CIESIN (2013). Columbia University and Information Technology Outreach Services - ITOS - University of Georgia. Global Roads Open Access Data Set, Version 1 (gROADSv1). Palisades, NY: NASA Socioecomonic Data and Applications Ceneter (SEDAC). http://sedac.ciesin.columbia.edu/data/set/groads-global-roads-open-access-v1. [Accessed on 03/01/14].

Chang, J., Chen, D., Ye, X., Li, S., Liang, W., Zhang, Z., \& Li, M. (2012). Coupling genetic and species distribution models to examine the response of the Hainan partridge (Arborophila ardens) to Late Quaternary climate. PloS one, 7(11), e50286.

Chen, I.C., Hill, J.K., Ohlemüller, R., Roy, D.B., \& Thomas, C.D. (2011). Rapid range shifts of species associated with high levels of 2 climate warming. Science, 333 (6045), 1024-1026.

Chetkiewicz, C.L.B., St Clair, C.C. \& Boyce, M.S. (2006) Corridors for conservation: integrating pattern and process. Annual Review of Ecology, Evolution, and Systematics, 37, 317-342. 
Clark, J.S., Lewis, M., \& Horvath, L. (2001). Invasion by Extremes: Population Spread with Variation in Dispersal and Reproduction. The American Naturalist, 157(5), 537-554.

Codling, E.A., Plank, M.J., \& Benhamou, S. (2008). Random walk models in biology. Journal of the Royal Society Interface, 5(25), 813-834.

Cohen, J. (1988). Statistical power analysis for the behavioral sciences. Psychology Press.

Coladonato, M. (1992a). Liquidambar styraciflua. In: Fire Effects Information System, [Online]. U.S. Department of Agriculture, Forest Service, Rocky Mountain Research Station, Fire Sciences Laboratory (Producer). Available: http://www.fs.fed.us/database/feis/ [2016, March 29].

Coladonato, M. (1992b). Ulmus americana. In: Fire Effects Information System, [Online]. U.S. Department of Agriculture, Forest Service, Rocky Mountain Research Station, Fire Sciences Laboratory (Producer). Available: http://www.fs.fed.us/database/feis/ [2016, March 29].

Coladonato, M. (1993). Solidago canadensis. In: Fire Effects Information System, [Online]. U.S. Department of Agriculture, Forest Service, Rocky Mountain Research Station, Fire Sciences Laboratory (Producer). Available: http://www.fs.fed.us/database/feis/plants/forb/solcan/all.html [2016, March 29].

Conlisk, E., Lawson, D., Spyhard, A.D., Franklin, J., Flint, L., Flint, A., \& Regan, H.M., (2012). The roles of dispersal, fecundity, and predation in the 8 population persistence of an oak (Quercus engelmannii) under global change. PloS one 7 (5), e36391.

Cooper, A.B., \& Millspaugh, J.J. (1999). The application of discrete choice models to wildlife resource selection studies. Ecology, 80(2), 566-575.

Cope, A.B. (1993). Abies procera. In: Fire Effects Information System, [Online]. U.S. Department of Agriculture, Forest Service, Rocky Mountain Research Station, Fire Sciences Laboratory (Producer). Available: http://www.fs.fed.us/database/feis/ [2016, March 29].

Coulon, A., Morellet, N., Goulard, M., Cargnelutti, B., Angibault, J.M., \& Hewison, A.M. (2008). Inferring the effects of landscape structure on roe deer (Capreolus capreolus) movements using a step selection function. Landscape Ecology, 23(5), 603-614.

Courant, S., \& Fortin, D. (2012). Search efficiency of free-ranging plains bison for optimal food items. Animal Behaviour, 84(4), 1039-1049.

Critchfield, W.B., \& Little, E.L., Jr. (1966), Geographic distribution of the pines of the world: U.S. Department of Agriculture Miscellaneous Publication 991, p. 1-97. 
Crosetto, M., \& Tarantola, S. (2001). Uncertainty and sensitivity analysis: tools for GISbased model implementation. International Journal of Geographical Information Science, 15(5), 415-437.

Cunze, S., Heydel, F., \& Tackenberg, O. (2013). Are plant species able to keep pace with the rapidly changing climate? PloS one, 8(7), e67909.

Czúcz, B., Csecserits, A., Botta-Dukát, Z., Kröel-Dulay, G., Szabó, R., Horváth, F., \& Molnár, Z. (2011). An indicator framework for the climatic adaptive capacity of natural ecosystems. Journal of Vegetation Science, 22(4), 711-725.

D’Elia, J., Haig, S.M., Johnson, M., Marcot, B.G., \& Young, R. (2015). Activity-specific ecological niche models for planning reintroductions of California condors (Gymnogyps californianus). Biological Conservation, 184, 90-99.

Dambach, J. \& Rödder, D. (2011). Applications and future challenges in marine species distribution modeling. Aquatic Conservation: Marine and Freshwater Ecosystems, 21(1), 92-100.

Delattre, T., Vernon, P. \& Burel, F. (2013). An agri-environmental scheme enhances butterfly dispersal in European agricultural landscapes.Agriculture, ecosystems \& environment, 166, 102-109.

Dingle, H., \& Drake, V.A. (2007). What is migration?. Bioscience, 57(2), 113-121.

Duchesne, T., Fortin, D., \& Rivest, L.P. (2015). Equivalence between step selection functions and biased correlated random walks for statistical inference on animal movement. PloS one, 10(4), e0122947.

Edrén, S., Wisz, M.S., Teilmann, J., Dietz, R. \& Söderkvist, J. (2010). Modelling spatial patterns in harbour porpoise satellite telemetry data using maximum entropy. Ecography, 33(4), 698-708.

Elith, J., \& Graham, C.H., (2009). Do they? How do they? WHY do they differ? On finding reasons for differing performances of species distribution models. Ecography, 32(1), 66-77.

Elith, J., Kearney, M. \& Phillips, S. (2010), The art of modelling range-shifting species. Methods in Ecology and Evolution, 1, 330-342

Elith, J., \& Leathwick, J.R. (2009). Species distribution models: ecological explanation and prediction across space and time. Annual Review of Ecology, Evolution, and Systematics, 40(1), 677.

Ellis, A.M., Václavík, T., \& Meentemeyer, R.K. (2010). When is connectivity important? A case study of the spatial pattern of sudden oak death. Oikos, 119(3), 485-493.

Engler, R., Guisan, A., \& Rechsteiner, L. (2004). An improved approach for predicting the distribution of rare and endangered species from occurrence and pseudoabsence data. Journal of Applied Ecology, 41 (2), 263-274. 
Engler, R., \& Guisan, A. (2009). MigClim: predicting plant distribution and dispersal in a changing climate. Diversity and Distributions, 15 (4), 590-601.

Engler, R., Hordijk, W., \& Pellissier, L. (2013). MigClim: Implementing dispersal into species distribution models. $R$ package version, 1(6).

Escobar, L.E., Lira-Noriega, A., Medina-Vogel, G., \& Peterson, A.T. (2014). Potential for spread of the white-nose fungus (Pseudogymnoascus destructans) in the Americas: use of Maxent and NicheA to assure strict model transference. Geospatial health, 9(1), 221-229.

Esser, L.L. (1993a). Eucalyptus globulus. In: Fire Effects Information System, [Online]. U.S. Department of Agriculture, Forest Service, Rocky Mountain Research Station, Fire Sciences Laboratory (Producer). Available: http://www.fs.fed.us/database/feis/ [2016, March 29].

Esser, L.L. (1993b). Pinus quadrifolia. In: Fire Effects Information System, [Online]. U.S. Department of Agriculture, Forest Service, Rocky Mountain Research Station, Fire Sciences Laboratory (Producer). Available: http://www.fs.fed.us/database/feis/ [2016, March 29].

Esser, L.L. (1994). Geranium richardsonii. In: Fire Effects Information System, [Online]. U.S. Department of Agriculture, Forest Service, Rocky Mountain Research Station, Fire Sciences Laboratory (Producer). Available: http://www.fs.fed.us/database/feis/ [2016, March 29].

Fagan, W.F., Lewis, M.A., Auger-Méthé, M., Avgar, T., Benhamou, S., Breed, G., LaDage, L., Schlagel, U.E., Tang, W., Papastamatiou, Y.P., Forester, J. \& Mueller, T. (2013). Spatial memory and animal movement. Ecology letters, 16(10), 1316-1329.

Ficetola, G.F., Thuiller, W., \& Miaud, C. (2007). Prediction and validation of the potential global distribution of a problematic alien invasive species-the American bullfrog. Diversity and Distributions, 13(4), 476-485.

Fielding, A.H., \& Bell, J.F. (1997). A review of methods for the assessment of prediction errors in conservation presence/absence models. Environmental conservation, 24(1), 38-49.

Fordham, D.A., Akçakaya, H.R., Araújo, M.B., Keith, D.A., \& Brook, B.W. (2013). Tools for integrating range change, extinction risk and climate change information into conservation management. Ecography, 36(9), 956-964.

Forester, J.D., Im, H.K. \& Rathouz, P.J., (2009). Accounting for animal movement in estimation of resource selection functions: sampling and data analysis. Ecology, 90(12), 3554-3565. 
Fortin, D., Beyer, H.L., Boyce, M.S., Smith, D.W., Duchesne, T., \& Mao, J.S. (2005). Wolves influence elk movements: behavior shapes a trophic cascade in Yellowstone National Park. Ecology, 86(5), 1320-1330.

Fourcade, Y., Engler, J.O., Rödder, D., \& Secondi, J. (2014) Mapping Species Distributions with MAXENT Using a Geographically Biased Sample of Presence Data: A Performance Assessment of Methods for Correcting Sampling Bias, PLoS One, 9(5), e97122.

Frair, J.L., Fieberg, J., Hebblewhite, M., Cagnacci, F., DeCesare, N.J., \& Pedrotti, L. (2010). Resolving issues of imprecise and habitat-biased locations in ecological analyses using GPS telemetry data. Philosophical Transactions of the Royal Society of London B: Biological Sciences, 365(1550), 2187-2200.

Franklin, J. (1995). Predictive vegetation mapping: geographic modelling of biospatial patterns in relation to environmental gradients. Progress in Physical Geography, 19(4), 474-499.

Franklin, J. (2009). Mapping species distributions: spatial inference and prediction. Cambridge: Cambridge University Press.

Franklin, J. (2010). Moving beyond static species distribution models in support of conservation biogeography. Diversity and Distributions, 16(3), 321-330.

Fryer, J.L. (2007). Corylus cornuta. In: Fire Effects Information System, [Online]. U.S. Department of Agriculture, Forest Service, Rocky Mountain Research Station, Fire Sciences Laboratory (Producer). Available: http://www.fs.fed.us/database/feis/ [2016, March 29].

Fryer, J.L. (2008). Lithocarpus densiflorus. In: Fire Effects Information System, [Online]. U.S. Department of Agriculture, Forest Service, Rocky Mountain Research Station, Fire Sciences Laboratory (Producer). Available: http://www.fs.fed.us/database/feis/ [2016, March 29].

Fryer, J.L. (2014). Picea mariana. In: Fire Effects Information System, [Online]. U.S. Department of Agriculture, Forest Service, Rocky Mountain Research Station, Fire Sciences Laboratory (Producer). Available: http://www.fs.fed.us/database/feis/ [2016, March 29].

Fuller, R.M., Devereux, B.J., Gillings, S., Hill, R.A., \& Amable, G.S. (2007). Bird distributions relative to remotely sensed habitats in Great Britain: Towards a framework for national modelling. Journal of Environmental Management, 84, 586-605.

Fuller, R.M., Groom, G.B., Jones, A.R., \& Thomson, A.G. (1993). Land Cover Map 1990 ( $1 \mathrm{~km}$ percentage target class, GB). NERC Environmental Information Data Centre. doi:10.5285/0172cc8c-8b5c-46cf-b08a-785ab832e88c. 
Fuller, R.M., Smith, G.M., Sanderson, J.M., Hill, R.A., Thomson, A.G., Cox, R., Brown, N.J., Clarke, R.T., Rothery, P., \& Gerard, F.F. (2002). Land Cover Map 2000 (1 km percentage target class, GB). NERC Environmental Information Data Centre. doi:10.5285/d5ee5360-12c5-448c-9d2b-f5c941fe3948.

Gallardo, B., Errea, M.P., \& Aldridge, D.C. (2012). Application of bioclimatic models coupled with network 12 analysis for risk assessment of the killer shrimp, Dikerogammarus villosus, in Great Britain. Biological Invasions, 14 (6), 12651278.

Garner, K. L., Chang, M. Y., Fulda, M. T., Berlin, J. A., Freed, R. E., Soo-Hoo, M. M.,Revell, D.L., Ikegami, M., Flint, L.E., Flint, A.L., \& Kendall, B. E. (2015). Impacts of sea level rise and climate change on coastal plant species in the central California coast. PeerJ, 3, e958.

GBIF (2015a) GBIF.org (8th October 2015) GBIF Occurrence Download http://doi.org/10.15468/dl.xlvctu

GBIF (2015b) GBIF.org (8th October 2015) GBIF Occurrence Download http://doi.org/10.15468/dl.ksm9he

GBIF (2015c) GBIF.org (8th October 2015) GBIF Occurrence Download http://doi.org/10.15468/dl.sphwvu

GBIF (2015d) GBIF.org (8th October 2015) GBIF Occurrence Download http://doi.org/10.15468/dl.s95nga

GBIF (2015e) GBIF.org (8th October 2015) GBIF Occurrence Download http://doi.org/10.15468/dl.mqsyq4

GBIF (2016) GBIF.org (9th February 2016) GBIF Occurrence Download http://doi.org/10.15468/dl.ahjffh

Geary, M., Fielding, A.H., \& Marsden, S.J. (2013). Designing mosaic landscapes for Black Grouse Tetrao tetrix using multi-scaled models. Ibis, 155(4), 792-803.

Gibbons, D.W, Reid, J.B., \& Chapman, R.A. (1993). The new atlas of breeding birds in Britain and Ireland. Poyser, London.

Gillies, C.S., Beyer, H.L., \& St Clair, C.C. (2011). Fine-scale movement decisions of tropical forest birds in a fragmented landscape. Ecological Applications, 21(3), 944-954.

Graham, C.H., Elith, J., Hijmans, R.J., Guisan, A., Townsend Peterson, A., \& Loiselle, B.A. (2008). The influence of spatial errors in species occurrence data used in distribution models. Journal of Applied Ecology, 45(1), 239-247.

Griffith, R.S. (1991). Liriodendron tulipifera. In: Fire Effects Information System, [Online]. U.S. Department of Agriculture, Forest Service, Rocky Mountain 
Research Station, Fire Sciences Laboratory (Producer). Available: http://www.fs.fed.us/database/feis/ [2016, March 29].

Griffith, R.S. (1992a). Pinus monticola. In: Fire Effects Information System, [Online]. U.S. Department of Agriculture, Forest Service, Rocky Mountain Research Station, Fire Sciences Laboratory (Producer). Available: http://www.fs.fed.us/database/feis/ [2016, March 29].

Griffith, R.S. (1992b). Sequoia sempervirens. In: Fire Effects Information System, [Online]. U.S. Department of Agriculture, Forest Service, Rocky Mountain Research Station, Fire Sciences Laboratory (Producer). Available: http://www.fs.fed.us/database/feis/ [2016, March 29].

Grimm, V., Berger, U., Bastiansen, F., Eliassen, S., Ginot, V., Giske, J., Goss-Custard, J., Grand, T., Heinz, S.K., Huse, G., Huth, A., Jepsen, J.U., Jorgensen, C., Mooij, W.M., Muller, B., Pe'er, G., Piou, C., Railsback, S.F., Robbins, A.M., Robbins, M.M., Rossmanith, E., Ruger, N., Strand, E., Souissi, S., Stillman, R.A., Vabo, R., Visser, U., \& DeAngelis, D. L. (2006). A standard protocol for describing individual-based and agent-based models. Ecological modelling, 198(1), 115-126.

Grimm, V., Berger, U., DeAngelis, D.L., Polhill, J.G., Giske, J., \& Railsback, S.F. (2010). The ODD protocol: a review and first update. Ecological modelling, 221(23), 2760-2768.

Gschweng, M., Kalko, E.K., Berthold, P., Fiedler, W., \& Fahr, J. (2012). Multi-temporal distribution modelling with satellite tracking data: predicting responses of a longdistance migrant to changing environmental conditions.Journal of Applied Ecology, 49(4), 803-813.

Gucker, C.L. (2005a). Acacia greggii. In: Fire Effects Information System, [Online]. U.S. Department of Agriculture, Forest Service, Rocky Mountain Research Station, Fire Sciences Laboratory (Producer). Available: http://www.fs.fed.us/database/feis/ [2016, March 29].

Gucker, C.L. (2005b). Fraxinus nigra. In: Fire Effects Information System, [Online]. U.S. Department of Agriculture, Forest Service, Rocky Mountain Research Station, Fire Sciences Laboratory (Producer). Available: http://www.fs.fed.us/database/feis/ [2016, March 29].

Gucker, C.L. (2005c). Fraxinus pennsylvanica. In: Fire Effects Information System, [Online]. U.S. Department of Agriculture, Forest Service, Rocky Mountain Research Station, Fire Sciences Laboratory (Producer). Available: http://www.fs.fed.us/database/feis/ [2016, March 29].

Gucker, C.L. (2005d). Galium aparine. In: Fire Effects Information System, [Online]. U.S. Department of Agriculture, Forest Service, Rocky Mountain Research Station, Fire Sciences Laboratory (Producer). Available: http://www.fs.fed.us/database/feis/ []. 
Gucker, C.L. (2006a). Agave lechuguilla. In: Fire Effects Information System, [Online]. U.S. Department of Agriculture, Forest Service, Rocky Mountain Research Station, Fire Sciences Laboratory (Producer). Available: http://www.fs.fed.us/database/feis/ (2006, May 23).

Gucker, C.L. (2006b). Cercocarpus montanus. In: Fire Effects Information System, [Online]. U.S. Department of Agriculture, Forest Service, Rocky Mountain Research Station, Fire Sciences Laboratory (Producer). Available: http://www.fs.fed.us/database/feis/plants/shrub/cermon/all.html [ 2016, March 29].

Gucker, C.L. (2006c). Yucca brevifolia. In: Fire Effects Information System, [Online]. U.S. Department of Agriculture, Forest Service, Rocky Mountain Research Station, Fire Sciences Laboratory (Producer). Available: http://www.fs.fed.us/database/feis/plants/tree/yucbre/all.html [2016, March 29].

Gucker, C.L. (2007a). Pinus jeffreyi. In: Fire Effects Information System, [Online]. U.S. Department of Agriculture, Forest Service, Rocky Mountain Research Station, Fire Sciences Laboratory (Producer). Available: http://www.fs.fed.us/database/feis/ [2016, March 29].

Gucker, C.L. (2007b). Quercus garryana. In: Fire Effects Information System, [Online]. U.S. Department of Agriculture, Forest Service, Rocky Mountain Research Station, Fire Sciences Laboratory (Producer). Available: http://www.fs.fed.us/database/feis/ [2016, March 29].

Gucker, C.L. (2007c). Salix discolor. In: Fire Effects Information System, [Online]. U.S. Department of Agriculture, Forest Service, Rocky Mountain Research Station, Fire Sciences Laboratory (Producer). Available: http://www.fs.fed.us/database/feis/ [2016, March 29].

Gucker, C.L. (2008a). Tragopogon dubius. In: Fire Effects Information System, [Online]. U.S. Department of Agriculture, Forest Service, Rocky Mountain Research Station, Fire Sciences Laboratory (Producer). Available: http://www.fs.fed.us/database/feis/ [2016, March 29].

Gucker, C.L. (2008b). Verbascum thapsus. In: Fire Effects Information System, [Online]. U.S. Department of Agriculture, Forest Service, Rocky Mountain Research Station, Fire Sciences Laboratory (Producer). Available: http://www.fs.fed.us/database/feis/ [2016, March 29].

Gucker, C.L. (2009a). Cirsium palustre. In: Fire Effects Information System, [Online]. U.S. Department of Agriculture, Forest Service, Rocky Mountain Research Station, Fire Sciences Laboratory (Producer). Available: http://www.fs.fed.us/database/feis/ [2016, March 29].

Gucker, C.L. (2009b). Coronilla varia. In: Fire Effects Information System, [Online]. U.S. Department of Agriculture, Forest Service, Rocky Mountain Research 
Station, Fire Sciences Laboratory (Producer). Available: http://www.fs.fed.us/database/feis/ [2016, March 29].

Gucker, C.L. (2009c). Dioscorea spp. In: Fire Effects Information System, [Online]. U.S. Department of Agriculture, Forest Service, Rocky Mountain Research Station, Fire Sciences Laboratory (Producer). Available: http://www.fs.fed.us/database/feis/ [2016, March 29].

Gucker, C.L. (2009d). Dipsacus fullonum, D. laciniatus. In: Fire Effects Information System, [Online]. U.S. Department of Agriculture, Forest Service, Rocky Mountain Research Station, Fire Sciences Laboratory (Producer). Available: http://www.fs.fed.us/database/feis/ [2016, March 29].

Gucker, C.L. (2009e). Heracleum mantegazzianum. In: Fire Effects Information System, [Online]. U.S. Department of Agriculture, Forest Service, Rocky Mountain Research Station, Fire Sciences Laboratory (Producer). Available: http://www.fs.fed.us/database/feis/ [2016, March 29].

Gucker, C.L. (2010a). Euphorbia esula. In: Fire Effects Information System, [Online]. U.S. Department of Agriculture, Forest Service, Rocky Mountain Research Station, Fire Sciences Laboratory (Producer). Available: http://www.fs.fed.us/database/feis/ [ 2016, March 29].

Gucker, C.L. (2010b). Populus alba and hybrids. In: Fire Effects Information System, [Online]. U.S. Department of Agriculture, Forest Service, Rocky Mountain Research Station, Fire Sciences Laboratory (Producer). Available: http://www.fs.fed.us/database/feis/ [2016, March 29].

Gucker, C.L. (2011). Quercus macrocarpa. In: Fire Effects Information System, [Online]. U.S. Department of Agriculture, Forest Service, Rocky Mountain Research Station, Fire Sciences Laboratory (Producer). Available: http://www.fs.fed.us/database/feis/ [2016, March 29].

Guisan, A. \& Thuiller, W. (2005). Predicting species distribution: offering more than simple habitat models. Ecology Letters, 8(9), 993-1009.

Guisan, A., \& Zimmermann, N. E. (2000). Predictive habitat distribution models in ecology. Ecological modeling, 135(2), 147-186

Habeck, R.J. (1992a). Pinus lambertiana. In: Fire Effects Information System, [Online]. U.S. Department of Agriculture, Forest Service, Rocky Mountain Research Station, Fire Sciences Laboratory (Producer). Available: http://www.fs.fed.us/database/feis/ [2016, March 29].

Habeck, R.J. (1992b). Pinus ponderosa var. benthamiana, P. p. var. ponderosa. In: Fire Effects Information System, [Online]. U.S. Department of Agriculture, Forest Service, Rocky Mountain Research Station, Fire Sciences Laboratory (Producer). Available: http://www.fs.fed.us/database/feis/plants/tree/pinponp.all.html [2016, March 29]. 
Habeck, R.J. (1992c). Sequoiadendron giganteum. In: Fire Effects Information System, [Online]. U.S. Department of Agriculture, Forest Service, Rocky Mountain Research Station, Fire Sciences Laboratory (Producer). Available: http://www.fs.fed.us/database/feis/ [2016, March 29].

Hall, L.S., Krausman, P.R. \& Morrison, M.L. (1997). The habitat concept and a plea for standard terminology. Wildlife Society Bulletin, 173-182.

Hamann, A., \& Aitken, S.N. (2013). Conservation planning under climate change: accounting for adaptive potential and migration capacity in species distribution models. Diversity and Distributions, 19(3), 268-280.

Hansson, L.A., and Åkesson, S. (Eds.). (2014). Animal movement across scales. Oxford University Press, USA.

Harris, H.T. (1990). Populus balsamifera subsp. balsamifera. In: Fire Effects Information System, [Online]. U.S. Department of Agriculture, Forest Service, Rocky Mountain Research Station, Fire Sciences Laboratory (Producer). Available: http://www.fs.fed.us/database/feis/ [2016, March 29.

Hauser, A.S. (2008). Pinus resinosa. In: Fire Effects Information System, [Online]. U.S. Department of Agriculture, Forest Service, Rocky Mountain Research Station, Fire Sciences Laboratory (Producer). Available: http://www.fs.fed.us/database/feis/ [2016, March 29].

Heffernan, J.B., Soranno, P.A., Angilletta Jr, M.J., Buckley, L.B., Gruner, D.S., Keitt, T.H., Kellner, J.R., Kominoski, J.S., Rocha, A.V., Xiao, J., Harms, T.K., Goring, S.J., Koenig, L.E., McDowell, W.H., Powell, H., Richardson, A.D., Stow, C.A., Vargas, R., \& Harms, T. K. (2014). Macrosystems ecology: understanding ecological patterns and processes at continental scales.Frontiers in Ecology and the Environment, 12(1), 5-14.

Hefley, T.J., Baasch, D.M., Tyre, A.J., \& Blankenship, E.E. (2015). Use of opportunistic sightings and expert knowledge to predict and compare Whooping Crane stopover habitat. Conservation Biology, 29 (5), 1337-1346.

Herrera, F.F. (2003). Distribución actualizada de las colonias de guacharos (Steatornis caripensis) en Venezuela. Bol Soc Venezol Espeleol, 37, 31-40.

Hijmans, R.J., Cameron, S.E., Parra, J.L., Jones, P.G., \& Jarivs, A. (2005). Very high resolution interpolated climate surfaces for global land areas. International Journal of Climatology, 25, 1965-1978.

Hijmans, R.J. \& Graham, C.H. (2006). The ability of climate envelope models to predict the effect of climate change on species distributions. Global change biology, 12(12), 2272-2281.

Hill, M.J., Braaten, R., Veitch, S.M., Lees, B.G., \& Sharma, S. (2005). Multi-criteria decision analysis in spatial decision support: the ASSESS analytic hierarchy 
process and the role of quantitative methods and spatially explicit analysis. Environmental Modelling and Software, 20, 955-976.

Hill, M.P., Chown, S.L., \& Hoffmann, A.A. (2013). A predicted niche shift corresponds with increased thermal resistance in an invasive mite, Halotydeus destructor. Global Ecology and Biogeography, 22(8), 942-951.

Hirzel, A.H., Le Lay, G., Helfer, V., Randin, C. \& Guisan, A. (2006). Evaluating the ability of habitat suitability models to predict species presences. Ecological Modelling, 199(2), 142-152.

Hjermann, D.Ø. (2000). Analyzing habitat selection in animals without well-defined home ranges. Ecology 81, 1462-1468.

Holland, R.A., Wikelski, M., Kümmeth, F., \& Bosque, C. (2009). The secret life of oilbirds: new insights into the movement ecology of a unique avian frugivore. PLoS One, 4(12), e8264.

Holloway, P., \& Miller, J.A. (2014). Uncertainty analysis of step-selection functions: The effect of model parameters on inferences about the relationship between animal movement and the environment. Lecture Notes in Computer Science, 8728, 48-63.

Holloway, P., Miller, J.A., \& Gillings, S. (2016). Incorporating movement in species distribution modelling: how do simulations of dispersal affect the accuracy and uncertainty of projections? DOI: 10.1080/13658816.2016.1158823.

Holyoak, M., Casagrandi, R., Nathan, R., Revilla, E., \& Spiegel, O. (2008). Trends and missing parts in the study of movement ecology. Proceedings of the National Academy of Sciences, 105(49), 19060-19065.

Howard, J.L. (1992a). Pseudotsuga macrocarpa. In: Fire Effects Information System, [Online].U.S. Department of Agriculture, Forest Service, Rocky Mountain Research Station, Fire Sciences Laboratory (Producer). Available: http://www.fs.fed.us/database/feis/ [2016, March 29].

Howard, J.L. (1992b). Washingtonia filifera. In: Fire Effects Information System, [Online]. U.S. Department of Agriculture, Forest Service, Rocky Mountain Research Station, Fire Sciences Laboratory (Producer). Available: http://www.fs.fed.us/database/feis/ [2016, March 29].

Howard, J.L. (1996). Populus tremuloides. In: Fire Effects Information System, [Online]. U.S. Department of Agriculture, Forest Service, Rocky Mountain Research Station, Fire Sciences Laboratory (Producer). Available: http://www.fs.fed.us/database/feis/ [2016, March 29].

Howard, J.L. (1999). Artemisia tridentata subsp. wyomingensis. In: Fire Effects Information System, [Online]. U.S. Department of Agriculture, Forest Service, Rocky Mountain Research Station, Fire Sciences Laboratory (Producer). Available: http://www.fs.fed.us/database/feis/ [2016, March 29]. 
Howard, J.L. (2003). Pinus ponderosa var. brachyptera, P. p. var. scopulorum. In: Fire Effects Information System, [Online]. U.S. Department of Agriculture, Forest Service, Rocky Mountain Research Station, Fire Sciences Laboratory (Producer). Available: http://www.fs.fed.us/database/feis/plants/tree/pinpons/all.html [2016, March 29].

Hsu, R.C.C., Tamis, W.L., Raes, N., de Snoo, G.R., Wolf, J.H., Oostermeijer, G., \& Lin, S.H. (2012). Simulating climate change impacts on forests and associated vascular epiphytes in a subtropical island of East Asia. Diversity and distributions, 18(4), 334-347.

Hu, J., \& Jiang, Z. (2011). Climate Change Hastens the Conservation of Urgency of an Endangered Ungulate, PLoS One, 6(8), e22873.

Innes, R.J. (2009). Paulownia tomentosa. In: Fire Effects Information System, [Online]. U.S. Department of Agriculture, Forest Service, Rocky Mountain Research Station, Fire Sciences Laboratory (Producer). Available: http://www.fs.fed.us/database/feis/ [2016, March 29].

Innes, R.J. (2010). Flourensia cernua. In: Fire Effects Information System, [Online]. U.S. Department of Agriculture, Forest Service, Rocky Mountain Research Station, Fire Sciences Laboratory (Producer). Available: http://www.fs.fed.us/database/feis/ [2016, March 29].

Innes, R.J. (2011). Tussilago farfara. In: Fire Effects Information System, [Online]. U.S. Department of Agriculture, Forest Service, Rocky Mountain Research Station, Fire Sciences Laboratory (Producer). Available: http://www.fs.fed.us/database/feis/ [2016, March 29].

Inoue, K., Lang, B.K., \& Berg, D.J. (2015). Past climate change drives current genetic structure of an endangered freshwater mussel species. Molecular ecology, 24(8), 1910-1926.

Iverson, L.R., Prasad, A.M., Matthews, S.N., \& Peters, M.P. (2011) Lessons learned while integrating habitat, dispersal, disturbance, and life-history traits into species habitat models under climate change. Ecosystems 14(6): 1005-1020.

Jønsson, K.A., Tøttrup, A.P., Borregaard, M.K., Keith, S.A., Rahbek, C., \& Thorup, K. (2016). Tracking Animal Dispersal: From Individual Movement to Community Assembly and Global Range Dynamics. Trends in ecology \& evolution, 31(3), 204-214.

Kays, R., Crofoot, M.C., Jetz, W., \& Wikelski, M. (2015). Terrestrial animal tracking as an eye on life and planet. Science, 348(6240), aaa2478.

Kissling, W.D., Field, R. \& Böhning-Gaese, K. (2008). Spatial patterns of woody plant and bird diversity: functional relationships or environmental effects?. Global Ecology and Biogeography, 17(3), 327-339. 
Land Processes Distributed Active Archive Center (LP DAAC) (2010), MODIS Land Cover Type Yearly L3 Global 500m SIN Grid, NASA EOSDIS Land Processes DAAC, USGS/Earth Resources Observation and Science (EROS) Center, Siox Falls, South Dakota (https://lpdaac.usgs.gov), accessed July 15, 2012, at https://lpdaac.usgs.gov/dataset_discovery/modis/modis products table/mcd12q1.

Latham, A.D.M., Latham, M.C., Boyce, M.S., \& Boutin, S. (2011). Movement responses by wolves to industrial linear features and their effect on woodland caribou in northeastern Alberta. Ecological Applications, 21(8), 2854-2865.

Lawler, J.J., White, D., Neilson, R.P., \& Blaustein, A.R. (2006). Predicting climateinduced range shifts: model differences and model reliability. Global Change Biology, 12(8), 1568-1584.

League, K.R. (2005a). Ceanothus cuneatus. In: Fire Effects Information System, [Online]. U.S. Department of Agriculture, Forest Service, Rocky Mountain Research Station, Fire Sciences Laboratory (Producer. Available: http://www.fs.fed.us/database/feis/plants/shrub/ceacun/all.html [2016, March 29].

League, K.R. (2005b). Kalmia latifolia. In: Fire Effects Information System, [Online]. U.S. Department of Agriculture, Forest Service, Rocky Mountain Research Station, Fire Sciences Laboratory (Producer). Available: http://www.fs.fed.us/database/feis/ [].

Lehner, B., Verdin, K., \& Jarvis, A. (2008). New global hydrology derived from spaceborne elevation data. Eos, Transactions, AGU, 89(10), 93-94.

Lele, S.R., \& Keim, J.L. (2006). Weighted distributions and estimation of resource selection probability functions. Ecology, 87(12), 3021-3028.

Levin, S.A. (1992). The problem of pattern and scale in ecology: the Robert $\mathrm{H}$. MacArthur award lecture. Ecology, 73(6), 1943-1967.

Little, E.L., Jr. (1971), Atlas of United States trees, volume 1, conifers and important hardwoods: U.S. Department of Agriculture Miscellaneous Publication 1146, 9 p., 200 maps.

Little, E.L., Jr. (1976), Atlas of United States trees, volume 3, minor Western hardwoods: U.S. Department of Agriculture Miscellaneous Publication 1314, 13 p., 290 maps.

Little, E.L., Jr. (1977), Atlas of United States trees, volume 4, minor Eastern hardwoods: U.S. Department of Agriculture Miscellaneous Publication 1342, 17 p., 230 maps.

Little, E.L., Jr. (1978), Atlas of United States trees, volume 5, Florida: U.S. Department of Agriculture Miscellaneous Publication 1361, 262 maps.

Liu, C., Berry, P.M., Dawson, T.P., \& Pearson, R.G. (2005). Selecting thresholds of occurrence in the prediction of species distributions. Ecography, 28 (3), 385-393. 
Lomolino, M.V., \& Heaney, L.R. (Eds.). (2004). Frontiers of biogeography: new directions in the geography of nature. Sunderland, MA: Sinauer Associates.

Lomolino, M.V., Sax, D.F., \& Brown, J.H. (2006) Foundations of Biogeography: University of Chicago Press.

Luo, Z., Jiang, Z., \& Tang, S. (2015). Impacts of climate change on distributions and diversity of ungulates on the Tibetan Plateau. Ecological Applications, 25(1), 2438.

Manly, B.F.L., McDonald, L., Thomas, D., McDonald, T.L., \& Erickson, W.P. (2002). Resource selection by animals: statistical design and analysis for field studies. Springer Science \& Business Media.

Marquiss, M., Petty, S.J., Anderson, D.I.K., \& Legge, G. (2003). Contrasting population trends of the Northern Goshawk (Accipter gentilis) in the Scottish/English Borders and north-east Scotland. In Birds of Prey in a Changing Environment (eds Thompson, D.B.A, Redpath, S.M., Fielding, A.H., Marquiss, M. and Galbraith, C.A.). The Stationery Office, Edinburgh.

Martin, R.D., Brabyn, L., \& Potter, M.A. (2011). Sensitivity of GIS-derived terrain variables at multiple scales for modelling stoat (Mustela erminea) activity. Applied Geography, 31(2), 770-779.

Martínez, I., González-Taboada, F., Wiegand, T., Camarero, J.J., \& Gutiérrez, E. (2012). Dispersal limitation and spatial scale affect model based projections of Pinus uncinata response to climate change in the Pyrenees. Global Change Biology, 18(5), 1714-1724.

Massimino, D., Johnston, A., Noble, D.G., \& Pearce-Higgins, J.W. (2015). Multi-species spatially-explicit indicators reveal spatially structured trends in bird communities. Ecological Indicators, 58, 277-285.

Mateo, R.G., Broennimann, O., Petitpierre, B., Muñoz, J., van Rooy, J., Laenen, B., Guisan, A., \& Vanderpoorten, A. (2015). What is the potential of spread in invasive bryophytes? Ecography, 38(5), 480-487.

Matthiopoulos, J. (2003). The use of space by animals as a function of accessibility and preference. Ecological Modelling, 159(2), 239-268.

Maude, G. (2010). The spatial ecology and foraging behavior of the brown hyaena (Hyaena brunnea) (Doctoral dissertation, University of Bristol).

McQuillan, M.A., \& Rice, A.M. (2015). Differential effects of climate and species interactions on range limits at a hybrid zone: potential direct and indirect impacts of climate change. Ecology and evolution, 5(21), 5120-5137.

McWilliams, J. (2003). Artemisia filifolia In: Fire Effects Information System, [Online]. U.S. Department of Agriculture, Forest Service, Rocky Mountain Research 
Station, Fire Sciences Laboratory (Producer). Available: http://www.fs.fed.us/database/feis/ [2016, March 29].

McWilliams, J. (2004). Sonchus arvensis. In: Fire Effects Information System, [Online]. U.S. Department of Agriculture, Forest Service, Rocky Mountain Research Station, Fire Sciences Laboratory (Producer). Available: http://www.fs.fed.us/database/feis/ [2016, March 29].

Medley, K.A. (2010). Niche shifts during the global invasion of the Asian tiger mosquito, Aedes albopictus Skuse (Culicidae), revealed by reciprocal distribution models. Global Ecology and Biogeography, 19 (1), 122-133.

Meentemeyer, R.K., Rank, N.E., Anacker, B.L., Rizzo, D.M., \& Cushman, J.H. (2008). Influence of land-cover change on the spread of an invasive forest pathogen. Ecological Applications, 18(1), 159-171.

Meineri, E., Skarpaas, O., \& Vandvik, V. (2012). Modeling alpine plant distributions at the landscape scale: Do biotic interactions matter? Ecological Modelling, 231, 110.

Mellick, R., Wilson, P.D., \& Rossetto, M. (2014). Demographic history and niche conservatism of tropical rainforest trees separated along an altitudinal gradient of a biogeographic barrier. Australian Journal of Botany, 62(5), 438-450.

Meyer, R. (2006). Lupinus perennis. In: Fire Effects Information System, [Online]. U.S. Department of Agriculture, Forest Service, Rocky Mountain Research Station, Fire Sciences Laboratory (Producer). Available: http://www.fs.fed.us/database/feis/ [2016, March 29].

Meyer, R. (2011). Ceanothus leucodermis. In: Fire Effects Information System, [Online]. U.S. Department of Agriculture, Forest Service, Rocky Mountain Research Station, Fire Sciences Laboratory (Producer). Available: http://www.fs.fed.us/database/feis/ [2016, March 29].

Midgley, G.F., Davies, I.D., Albert, C.H., Altwegg, R., Hannah, L., Hughes, G.O., O'Halloran, L.R., Seo, C., Thorne, J.H., \& Thuiller, W. (2010). BioMove-an integrated platform simulating the dynamic response of species to environmental change. Ecography, 33(3), 612-616.

Midgley, G.F., Hughes, G.O., Thuiller, W., \& Rebelo, A.G. (2006). Migration rate limitations on climate change-induced range shifts in Cape Proteaceae. Diversity and Distributions, 12, 555-562.

Miller, J.A. (2015). Towards a better understanding of dynamic interaction metrics for wildlife: a null model approach. Transactions in GIS, 19(3), 342-361.

Miller, J.A., \& Hanham, R.Q. (2011). Spatial nonstationarity and the scale of speciesenvironment relationships in the Mojave Desert, California, USA. International Journal of Geographical Information Science, 25(3), 423-438. 
Miller, J. A., \& Holloway, P. (2015). Incorporating movement in species distribution models. Progress in Physical Geography, 39(6), 837-849.

Moenickes, S., Frassl, M., Schlief, J., Kupisch, M., Mutz, M., Suhling, F., \& Richter, O. (2012). Temporal patterns of populations in a warming world: a modelling framework. Marine Biology, 159(11), 2605-2620.

Morton, R.D., Rowland, C.S.; Wood, C.M., Meek, L., Marston, C.G., \& Smith, G.M. (2014). Land Cover Map 2007 (1 km percentage target class, GB) v1.2. NERC Environmental Information Data Centre. doi:10.5285/fdf8c8d3-5998-45a5-84317f5e6302fc32.

Movebank, Data from: The secret life of oilbirds: new insights into the movement ecology of a unique avian frugivore, http://hdl.handle.net/10255/move.269

Munger, G.T. (2001). Alliaria petiolata. In: Fire Effects Information System, [Online]. U.S. Department of Agriculture, Forest Service, Rocky Mountain Research Station, Fire Sciences Laboratory (Producer). Available: http://www.fs.fed.us/database/feis/ [2016, March 29].

Munger, G.T. (2002). Lythrum salicaria. In: Fire Effects Information System, [Online]. U.S. Department of Agriculture, Forest Service, Rocky Mountain Research Station, Fire Sciences Laboratory (Producer). Available: http://www.fs.fed.us/database/feis/ [2016, March 29].

Munger, G.T. (2005). Melaleuca quinquenervia. In: Fire Effects Information System, [Online]. U.S. Department of Agriculture, Forest Service, Rocky Mountain Research Station, Fire Sciences Laboratory (Producer). Available: http://www.fs.fed.us/database/feis/plants/tree/melqui/all.html [2016, March 29].

Nathan, R., Getz, W.M., Revilla, E., Holyoak, M., Kadmon, R., Saltz, D., \& Smouse, P.E. (2008a). A movement ecology paradigm for unifying organismal movement research. Proceedings of the National Academy of Sciences, 105(49), 1905219059.

Nathan, R., Schurr, F.M., Spiegel, O., Steinitz, O., Trakhtenbrot, A., \& Tsoar, A. (2008b). Mechanisms of long-distance seed dispersal. Trends in Ecology \& Evolution, 23(11), 638-647.

Northrup, J.M., Hooten, M.B., Anderson Jr, C.R., \& Wittemyer, G. (2013). Practical guidance on characterizing availability in resource selection functions under a use-availability design. Ecology, 94(7), 1456-1463.

Northrup, J.M., Pitt, J., Muhly, T.B., Stenhouse, G.B., Musiani, M., \& Boyce, M.S. (2012). Vehicle traffic shapes grizzly bear behaviour on a multiple-use landscape. Journal of Applied Ecology, 49(5), 1159-1167. 
O’Connor, M.P., Juanes, F., McGarigal, K., \& Caris, J. (2012). Describing juvenile American shad and striped bass habitat use in the Hudson River Estuary using species distribution models. Ecological Engineering, 48, 101-108.

Oliveira-Santos, L.G.R., Forester, J.D., Piovezan, U., Tomas, W.M. \& Fernandez, F.A. (2016). Incorporating animal spatial memory in step selection functions. Journal of Animal Ecology.

O’Sullivan, D. \& Perry, G.L.W. (2013). Spatial Simulation: Exploring Pattern and Process. Wiley-Blackwell: Oxford, UK.

Paradis, E., Baillie, S.R., Sutherland, W.J., \& Gregory, R.D. (1998). Patterns of natal and breeding dispersal in birds. Journal of Animal Ecology, 67(4), 518-536.

Pavek, D.S. (1992). Halogeton glomeratus. In: Fire Effects Information System, [Online]. U.S. Department of Agriculture, Forest Service, Rocky Mountain Research Station, Fire Sciences Laboratory (Producer). Available: http://www.fs.fed.us/database/feis/ [2016, March 29].

Pearson, R.G. \& Dawson, T.P. (2003). Predicting the impacts of climate change on the distribution of species: are bioclimate envelope models useful?. Global ecology and biogeography, 12(5), 361-371.

Pereboom, V., Mergey, M., Villerette, N., Helder, R., Gerard, J.F., \& Lode, T. (2008). Movement patterns, habitat selection, and corridor use of a typical woodlanddweller species, the European pine marten (Martes martes), in fragmented landscape. Canadian Journal of Zoology, 86(9), 983-991.

Perry, M., \& Hollis, D. (2005a). The development of a new set of long-term climate averages for the UK. International Journal of Climatology, 25, 1023-1039.

Perry, M., \& Hollis, D. (2005b). The generation of monthly gridded datasets for a range of climatic variables over the UK. International Journal of Climatology, 25, 1041-1054.

Peters, R.H. (1983). The Ecological Implications of Body Size. Cambridge University Press, Cambridge.

Peters, D.P.C., Bestelmeyer, B.T., \& Turner, M.G. (2007). Cross-scale interactions and changing pattern-process relationships: consequences for system dynamics. Ecosystems 10, 790-96.

Peterson, A.T., Martínez-Meyer, E., \& González-Salazar, C. (2004). Reconstructing the Pleistocene geography of the Aphelocoma jays (Corvidae). Diversity and Distributions, 10(4), 237-246.

Peterson, A.T., Papeş, M., \& Eaton, M. (2007). Transferability and model evaluation in ecological niche modeling: a comparison of GARP and Maxent. Ecography, 30(4), 550-560. 
Peterson, A. T., Soberón, J., Pearson, R. G., Anderson, R. P., Martínez-Meyer, E., Nakamura, M., \& Araújo, M. B. (2011). Ecological niches and geographic distributions. Princeton University Press.

Peterson, A.T., \& Vieglais, D.A. (2001). Predicting Species Invasions Using Ecological Niche Modeling: New Approaches from Bioinformatics Attack a Pressing Problem A new approach to ecological niche modeling, based on new tools drawn from biodiversity informatics, is applied to the challenge of predicting potential species' invasions. BioScience, 51(5), 363-371.

Phillips, S.J., \& Dudík, M. (2008). Modeling of species distributions with Maxent: new extensions and a comprehensive evaluation. Ecography, 31 (2), 161-175.

Phillips, S.J., Anderson, R.P., \& Schapire, R.E. (2006). Maximum entropy modeling of species geographic distributions. Ecological modelling, 190(3), 231-259.7

Pikesley, S.K., Broderick, A.C., Cejudo, D., Coyne, M.S., Godfrey, M.H., Godley, B.J., Lopez, P., López-Jurado, L.F., Elsy Merino, S., Varo-Cruz, N. \& Witt, M.J. (2015). Modelling the niche for a marine vertebrate: a case study incorporating behavioural plasticity, proximate threats and climate change. Ecography, 38(8), 803-812.

Pittiglio, C., Skidmore, A.K., van Gils, H.A., \& Prins, H.H. (2012). Identifying transit corridors for elephant using a long time-series. International journal of applied earth observation and geoinformation, 14(1), 61-72.

Plank, J.A., \& Edwards, A.M. (2011). Assessing Lévy walks as models of animal foraging. Journal of the Royal Society Interface, 8(62), 1233-1247.

Pounds, J.A. (2001). Climate and amphibian declines. Nature, 410(6829), 639-640.

Qiao, H., Soberón, J., \& Peterson, A.T. (2015). No silver bullets in correlative ecological niche modelling: insights from testing among many potential algorithms for niche estimation. Methods in Ecology and Evolution, 6(10), 1126-1136.

R Development Core Team (2008). R: A language and environment for statistical computing. R Foundation for Statistical Computing, Vienna, Austria ISBN 3900051-07-0, URL http://www.R-project.org.

Ramankutty, N., Evan, A.T., Mondreda, C., \& Foley, J.A. (2010). Global Agricultural Lands: Croplands, 2000. Data distributed by the Socioeconomic Data and Applications Center (SEDAC): http://seda.ciesin.columbia.edu/es/algands.html. [Accessed 03/01/14].

Ramos-Fernádez, G., Mateos, J.L., Miramontes, O., Cocho, O., Larralde, H., \& AyalaOrozco, B. (2004). Lévy walk patterns in the foraging movements of spider monkeys (Ateles geoffroyi). Behavioral Ecology and Sociobiology 55, 223-230.

Raxworthy, C.J., Pearson, R.G., Zimkus, B.M., Reddy, S., Deo, A.J., Nussbaum, R.A., \& Ingram, C.M. (2008). Continental speciation in the tropics: contrasting 
biogeographic patterns of divergence in the Uroplatus leaf-tailed gecko radiation of Madagascar. Journal of Zoology, 275(4), 423-440.

Ray, N., Lehmann, A., \& Joly, P. (2002). Modeling spatial distribution of amphibian populations: a GIS approach based on habitat matrix permeability. Biodiversity \& Conservation, 11(12), 2143-2165.

Razgour, O. (2015). Beyond species distribution modeling: A landscape genetics approach to investigating range shifts under future climate change. Ecological Informatics, 30, 250-256.

Reese, G.C., Wilson, K.R., Hoeting, J.A., \& Flather, C.H. (2005). Factors affecting species distribution predictions: a simulation modeling experiment. Ecological Applications, 15(2), 554-564.

Reeves, S.L. (2006). Lupinus latifolius. In: Fire Effects Information System, [Online]. U.S. Department of Agriculture, Forest Service, Rocky Mountain Research Station, Fire Sciences Laboratory (Producer). Available: http://www.fs.fed.us/database/feis/plants/forb/luplat/all.html [2016, March 29].

Richards, C.L., Carstens, B.C., \& Lacey Knowles, L. (2007). Distribution modelling and statistical phylogeography: an integrative framework for generating and testing alternative biogeographical hypotheses. Journal of Biogeography, 34(11), 18331845.

Richmond, O.M., McEntee, J.P., Hijmans, R.J., \& Brashares, J.S. (2010). Is the climate right for Pleistocene rewilding? Using species distribution models to extrapolate climatic suitability for mammals across continents. PloS one, 5(9), e12899.

Riordan, E.C., Gillespie, T.W., Pitcher, L., Pincetl, S.S., Jenerette, G.D., \& Pataki, D.E. (2015). Threats of future climate change and land use to vulnerable tree species native to Southern California. Environmental Conservation, 42(02), 127-138.

Ripple, W.J., \& Larsen, E.J. (2000). Historic aspen recruitment, elk, and wolves in northern Yellowstone National Park, USA. Biological Conservation, 95(3), 361370.

Robinson, R.A., (2005). BirdFacts: profiles of birds occurring in Britain and Ireland (BTO Research Report 407) [online]. BTO, Thetford. Available from: http://www.bto.org/birdfacts. Accessed 28/01/2015.

Robinson, R.A., \& Clark, J.A. (2014). The online ringing report: bird ringing in Britain and Ireland in 2013 [online]. Thetford: BTO. Available from: http://www.bto.org/ringing-report. Accessed 30/05/2015.

Roever, C. L., Boyce, M. S., \& Stenhouse, G. B. (2010). Grizzly bear movements relative to roads: application of step selection functions.Ecography, 33(6), 1113-1122. 
Sagarin, R.D., \& Gaines, S.D. (2002). Geographical abundance distributions of coastal invertebrates: using one-dimensional ranges to test biogeographic hypotheses. Journal of Biogeography, 29(8), 985-997.

Sahlean, T.C., Gherghel, I., Papeş, M., Strugariu, A., \& Zamfirescu, Ş.R. (2014). Refining climate change projections for organisms with low dispersal abilities: a case study of the Caspian whip snake. PloS one, 9(3), e91994.

Saupe, E.E., Barve, V., Myers, C.E., Soberón, J., Barve, N., Hensz, C.M., Peterson, A.T., Owens, H.L., \& Lira-Noriega, A. (2012). Variation in niche and distribution model performance: the need for a priori assessment of key causal factors. Ecological Modelling, 237, 11-22.

Sawyer, H., Nielson, R.M., Lindzey, F., \& McDonald, L.L. (2006). Winter habitat selection of mule deer before and during development of a natural gas field. Journal of Wildlife Management, 70(2), 396-403.

Scher, J.S. (2002). Larix occidentalis. In: Fire Effects Information System, [Online]. U.S. Department of Agriculture, Forest Service, Rocky Mountain Research Station, Fire Sciences Laboratory (Producer). Available: http://www.fs.fed.us/database/feis/ [2016, March 29].

Schymanski, S.J., Dormann, C.F., Cabral, J., Chuine, I., Graham, C.H., Hartig, F., Kearney, M., Morin, X., Romermann, C., Schroder, B., \& Singer, A. (2013). Process, correlation and parameter fitting in species distribution models: a response to Kriticos et al. Journal of biogeography, 40(3), 612-613.

Sclafani, C.J. (2006). Castilleja cinerea. In: Fire Effects Information System, [Online]. U.S. Department of Agriculture, Forest Service, Rocky Mountain Research Station, Fire Sciences Laboratory (Producer). Available: http://www.fs.fed.us/database/feis/plants/forb/cascin/all.html [2016, March 29].

Sharrock, J.T.R. (1976) The atlas of breeding birds in Britain and Ireland. T. \& A.D. Poyser.

Sibly, R.M., Grimm, V., Martin, B.T., Johnston, A.S., Kułakowska, K., Topping, C.J., Calow, P., Nabe-Neilsen, J., Thorbek, P., \& DeAngelis, D.L. (2013). Representing the acquisition and use of energy by individuals in agent-based models of animal populations. Methods in Ecology and Evolution, 4(2), 151-161

Sinclair, S.J., White, M.D. \& Newell, G.R. (2010). How useful are species distribution models for managing biodiversity under future climates. Ecology and Society, 15(1), 1-8.

Smart, L.S., Swenson, J.J., Christensen, N.L., \& Sexton, J.O. (2012). Three-dimensional characterization of pine forest type and red-cockaded woodpecker habitat by small-footprint, discrete-return lidar. Forest Ecology and Management, 281, 100110. 
Smith, F.D., May, R.M., Pellew, R., Johnson, T.H., \& Walter, K.R. (1993). How much do we know about the current extinction rate? Trends in Ecology and Evolution, 8 (10), 375-378.

Smouse, P. E., Focardi, S., Moorcroft, P. R., Kie, J. G., Forester, J. D., \& Morales, J. M. (2010). Stochastic modelling of animal movement. Philosophical Transactions of the Royal Society B: Biological Sciences, 365(1550), 2201-2211.

Soberón, J. (2007). Grinnellian and Eltonian niches and geographic distributions of species. Ecology letters, 10(12), 1115-1123.

Soberón, J. \& Peterson, A.T. (2005). Interpretation of models of fundamental ecological niches and species' distributional areas. Biodiversity Informatics, 2, 1-10.

South, A. (1999). Extrapolating from individual movement behaviour to population spacing patterns in a ranging mammal. Ecological Modelling, 117(2), 343-360.

Squires, J.R., DeCesare, N.J., Olson, L.E., Kolbe, J.A., Hebblewhite, M., \& Parks, S.A. (2013). Combining resource selection and movement behavior to predict corridors for Canada lynx at their southern range periphery. Biological Conservation, 157, 187-195.

Steinberg, P.D. (2002). Pseudotsuga menziesii var. glauca. In: Fire Effects Information System, [Online]. U.S. Department of Agriculture, Forest Service, Rocky Mountain Research Station, Fire Sciences Laboratory (Producer). Available: http://www.fs.fed.us/database/feis/ [2016, March 29].

Stone, K.R. (2010a). Hieracium aurantiacum. In: Fire Effects Information System, [Online]. U.S. Department of Agriculture, Forest Service, Rocky Mountain Research Station, Fire Sciences Laboratory (Producer). Available: http://www.fs.fed.us/database/feis/ [2016, March 29].

Stone, K.R. (2010b). Polygonum sachalinense, P. cuspidatum, P. $\times$ bohemicum. In: Fire Effects Information System, [Online]. U.S. Department of Agriculture, Forest Service, Rocky Mountain Research Station, Fire Sciences Laboratory (Producer). Available: http://www.fs.fed.us/database/feis/ [2016, March 29]

Strubbe, D., Beauchard, O., \& Matthysen, E. (2015). Niche conservatism among nonnative vertebrates in Europe and North America. Ecography, 38(3), 321-329.

Sullivan, J. (1992a). Geranium maculatum. In: Fire Effects Information System, [Online]. U.S. Department of Agriculture, Forest Service, Rocky Mountain Research Station, Fire Sciences Laboratory (Producer). Available: http://www.fs.fed.us/database/feis/ [2016, March 29].

Sullivan, J. (1992b). Sorbus americana. In: Fire Effects Information System, [Online]. U.S. Department of Agriculture, Forest Service, Rocky Mountain Research Station, Fire Sciences Laboratory (Producer). Available: http://www.fs.fed.us/database/feis/ [2016, March 29]. 
Sullivan, J. (1993a). Abies fraseri. In: Fire Effects Information System, [Online]. U.S. Department of Agriculture, Forest Service, Rocky Mountain Research Station, Fire Sciences Laboratory (Producer). Available: http://www.fs.fed.us/database/feis/ [2016, March 29].

Sullivan, J. (1993b). Picea rubens. In: Fire Effects Information System, [Online]. U.S. Department of Agriculture, Forest Service, Rocky Mountain Research Station, Fire Sciences Laboratory (Producer). Available: http://www.fs.fed.us/database/feis/ [2016, March 29].

Sullivan, J. (1993c). Pinus sylvestris. In: Fire Effects Information System, [Online]. U.S. Department of Agriculture, Forest Service, Rocky Mountain Research Station, Fire Sciences Laboratory (Producer). Available: http://www.fs.fed.us/database/feis/plants/tree/pinsyl/all.html [2016, March 29].

Sullivan, J. (1993d). Pinus virginiana. In: Fire Effects Information System, [Online]. U.S. Department of Agriculture, Forest Service, Rocky Mountain Research Station, Fire Sciences Laboratory (Producer). Available: http://www.fs.fed.us/database/feis/ [2016, March 29].

Sullivan, J. (1994). Picea abies. In: Fire Effects Information System, [Online]. U.S. Department of Agriculture, Forest Service, Rocky Mountain Research Station, Fire Sciences Laboratory (Producer). Available: http://www.fs.fed.us/database/feis/ [2016, March 29].

Sullivan, M.J.P., Davies, R.G., Reino, L., \& Franco, A.M.A. (2012). Using dispersal information to model the species-environment relationship of spreading nonnative species. Methods in Ecology and Evolution, 3, 870-879.

Summers, D.M., Bryan, B.A., Crossman, N.D., \& Meyer, W.S. (2012). Species vulnerability to climate change: impacts on spatial conservation priorities and species representation. Global Change Biology, 18(7), 2335-2348.

Sutherland, G.D., Harestad, A.S., Price, K., \& Lertzman, K.P. (2000). Scaling of natal dispersal distances in terrestrial birds and mammals. Conservation Ecology, 4(1), 16.

Tang, W., \& Bennett, D.A. (2010). Agent-based Modeling of Animal Movement: A Review. Geography Compass, 4(7), 682-700.

Ter Steege, H., Pitman, N.C., Sabatier, D., Baraloto, C., Salomao, R.P., Guevara, J.E., et al. (2013). Hyperdominance in the Amazonian tree flora. Science, 342(6156), 1243092 .

Tesky, J.L. (1992a). Thuja plicata. In: Fire Effects Information System, [Online]. U.S. Department of Agriculture, Forest Service, Rocky Mountain Research Station, Fire Sciences Laboratory (Producer). Available: http://www.fs.fed.us/database/feis/ [2016, March 29]. 
Tesky, J.L. (1992b). Tsuga heterophylla. In: Fire Effects Information System, [Online]. U.S. Department of Agriculture, Forest Service, Rocky Mountain Research Station, Fire Sciences Laboratory (Producer). Available: http://www.fs.fed.us/database/feis/ [2016, March 29].

Therneau, T. (2013). A package for survival analysis in S. R package version 2.37.4.

Thomas, C.D., Cameron, A., Green, R.E., Bakkenes, M., Beaumont, L.J., Collingham, Y.C., Erasmus, B.F., De Siqueira, M.F., Grainger, A., Hannah, L. \& Hughes, L. (2004). Extinction risk from climate change. Nature, 427(6970), 145-148.

Thuiller, W., Georges, D., \& Engler, R. (2013). biomod2: Ensemble platform for species distribution modeling. $R$ package version, 2(7), r560.

Thurfjell, H., Ciuti, S., \& Boyce, M.S. (2014). Applications of step-selection functions in ecology and conservation. Movement Ecology, 2(4).

Tirmenstein, D.A. (1991a). Acer saccharum. In: Fire Effects Information System, [Online]. U.S. Department of Agriculture, Forest Service, Rocky Mountain Research Station, Fire Sciences Laboratory (Producer). Available: http://www.fs.fed.us/database/feis/ [2016, March 29].

Tirmenstein, D. A. (1991b). Quercus rubra. In: Fire Effects Information System, [Online]. U.S. Department of Agriculture, Forest Service, Rocky Mountain Research Station, Fire Sciences Laboratory (Producer). Available: http://www.fs.fed.us/database/feis/ [2016, March 29].

Tirmenstein, D.A. (1999a). Artemisia tridentata subsp. tridentata. In: Fire Effects Information System, [Online]. U.S. Department of Agriculture, Forest Service, Rocky Mountain Research Station, Fire Sciences Laboratory (Producer). Available: http://www.fs.fed.us/database/feis/plants/shrub/arttrit/all.html [2016, March 29].

Tirmenstein, D.A. (1999b). Juniperus occidentalis. In: Fire Effects Information System, [Online]. U.S. Department of Agriculture, Forest Service, Rocky Mountain Research Station, Fire Sciences Laboratory (Producer). Available: http://www.fs.fed.us/database/feis/ [2016, March 29].

Tollefson, J.E. (2008). Calocedrus decurrens. In: Fire Effects Information System, [Online]. U.S. Department of Agriculture, Forest Service, Rocky Mountain Research Station, Fire Sciences Laboratory (Producer). Available: http://www.fs.fed.us/database/feis/plants/tree/caldec/all.html [2016, March 29].

Trainor, A.M., Schmitz, O.J., Ivan, J.S., \& Shenk, T.M. (2014). Enhancing species distribution modeling by characterizing predator-prey interactions. Ecological Applications, 24(1), 204-216. 
Turchin, P. (1998). Quantitative analysis of movement: measuring and modeling population redistribution in animals and plants (Vol. 1). Sunderland: Sinauer Associates.

Turner, M.G., Gardner, R.H. \& O'neill, R.V. (2001). Landscape ecology in theory and practice: pattern and process. Springer Science \& Business Media.

Uchytil, R.J. (1990). Prosopis velutina. In: Fire Effects Information System, [Online]. U.S. Department of Agriculture, Forest Service, Rocky Mountain Research Station, Fire Sciences Laboratory (Producer). Available: http://www.fs.fed.us/database/feis/ [2016, March 29].

Uchytil, R.J. (1991a). Abies balsamea. In: Fire Effects Information System, [Online]. U.S. Department of Agriculture, Forest Service, Rocky Mountain Research Station, Fire Sciences Laboratory (Producer). Available: http://www.fs.fed.us/database/feis/ [2016, March 29].

Uchytil, R.J. (1991b). Abies lasiocarpa. In: Fire Effects Information System, [Online]. U.S. Department of Agriculture, Forest Service, Rocky Mountain Research Station, Fire Sciences Laboratory (Producer). Available: http://www.fs.fed.us/database/feis/plants/tree/abilas/all.html [2016, March 29].

Uchytil, R.J. (1991c). Betula papyrifera. In: Fire Effects Information System, [Online]. U.S. Department of Agriculture, Forest Service, Rocky Mountain Research Station, Fire Sciences Laboratory (Producer). Available: http://www.fs.fed.us/database/feis/ [2016, March 29].

Uchytil, R.J. (1991d). Larix laricina. In: Fire Effects Information System, [Online]. U.S. Department of Agriculture, Forest Service, Rocky Mountain Research Station, Fire Sciences Laboratory (Producer). Available: http://www.fs.fed.us/database/feis/ [2016, March 29.

Uchytil, R.J. (1991e). Picea engelmannii. In: Fire Effects Information System, [Online]. U.S. Department of Agriculture, Forest Service, Rocky Mountain Research Station, Fire Sciences Laboratory (Producer). Available: http://www.fs.fed.us/database/feis/ [2016, March 29].

University of East Anglia Climatic Research Unit; Jones, P.D.; Harris, I. (2013): CRU TS3.10: Climatic Research Unit (CRU) Time-Series (TS) Version 3.10 of High Resolution Gridded Data of Month-by-month Variation in Climate (Jan. 1901 Dec. 2009). NCAS British Atmospheric Data Centre, Accessed 10/10/2015. http://catalogue.ceda.ac.uk/uuid/ac3e6be017970639a9278e64d3fd5508

Urban, M.C. (2015). Accelerating extinction risk from climate change. Science, 348(6234), 571-573.

Václavík, T., Kanaskie, A., Hansen, E.M., Ohmann, J.L., \& Meentemeyer, R.K. (2010). Predicting potential and actual distribution of sudden oak death in Oregon: 
Prioritizing landscape contexts for early detection and eradication of disease outbreaks. Forest Ecology and Management, 260(6), 1026-1035.

Václavík, T., Kupfer, J.A. \& Meentemeyer, R.K. (2012). Accounting for multi-scale spatial autocorrelation improves performance of invasive species distribution modelling (iSDM). Journal of Biogeography, 39(1), 42-55.

VanDerWal, J., Shoo, L.P., Johnson, C.N., \& Williams, S.E. (2009). Abundance and the environmental niche: Environmental suitability estimated from niche models predicts the upper limit of local abundance. American Naturalist, 174, 282-291.

Veloz, S.D., Williams, J.W., Blois, J.L., He, F., Otto-Bliesner, B. \& Liu, Z. (2012). Noanalog climates and shifting realized niches during the late quaternary: implications for 21 st-century predictions by species distribution models. Global Change Biology, 18(5), 1698-1713.

Vittoz, P., \& Engler, R. (2007). Seed dispersal distances: a typology based on dispersal modes and plant traits. Botanica Helvetica, 117(2), 109-124.

Warren, D.L., Glor, R.E., \& Turelli, M. (2008). Environmental niche equivalency versus conservatism: quantitative approaches to niche evolution. Evolution, 62, 28682883.

Wiens, J.A., Seavy, N.E., \& Jongsomjit, D. (2011). Protected areas in climate space: What will the future bring? Biological Conservation, 144 (8), 2119-2125.

Wilensky, U. (1997). NetLogo Wolf Sheep Predation model. http://ccl.northwestern.edu/netlogo/models/WolfSheepPredation. Center for Connected Learning and Computer-Based Modeling, Northwestern University, Evanston, IL

Wilensky, U. \& Reisman, K. (1999). Connected Science: Learning Biology through Constructing and Testing Computational Theories - an Embodied Modeling Approach. International Journal of Complex Systems, M. 234, 1 - 12.

Williams, J.W., Shuman, B.N., Webb III, T., Bartlein, P.J. \& Leduc, P.L. (2004). LateQuaternary vegetation dynamics in North America: scaling from taxa to biomes. Ecological Monographs, 74(2), 309-334.

Wisz, M. S., Pottier, J., Kissling, W. D., Pellissier, L., Lenoir, J., Damgaard, C. F., Dormann, C.F., Forchhammer, M.C., Grythnes, J.A., Guisan, A., Heikkinen, R.K., Hoye, T.T., Kuhn, I., Luoto, M., Maiornao, L., Nilsson, M.C., Normand, S., Ockinger, E., Schmidt, N.M., Termansen, M., Timmerman, A., Wardle, D.A., Aastrup, P., \& Svenning, J. C. (2013). The role of biotic interactions in shaping distributions and realised assemblages of species: implications for species distribution modelling. Biological Reviews, 88(1), 15-30. 
Wittwer, T., O'Hara, R.B., Caplat, P., Hickler, T., \& Smith, H.G. (2015). Long-term population dynamics of a migrant bird suggests interaction of climate change and competition with resident species. Oikos. 124(9), 1151-1159.

Zouhar, K.L. (2001). Pinus monophylla. In: Fire Effects Information System, [Online]. U.S. Department of Agriculture, Forest Service, Rocky Mountain Research Station, Fire Sciences Laboratory (Producer). Available: http://www.fs.fed.us/database/feis/ [2016, March 29].

Zouhar, K.L. (2002a). Carduus nutans. In: Fire Effects Information System, [Online]. U.S. Department of Agriculture, Forest Service, Rocky Mountain Research Station, Fire Sciences Laboratory (Producer). Available: http://www.fs.fed.us/database/feis/ [2016, March 29].

Zouhar, K.L. (2002b). Centaurea solstitialis. In: Fire Effects Information System, [Online]. U.S. Department of Agriculture, Forest Service, Rocky Mountain Research Station, Fire Sciences Laboratory (Producer). Available: http://www.fs.fed.us/database/feis/ [2016, March 29].

Zouhar, K.L. (2002c). Cirsium vulgare. In: Fire Effects Information System, [Online]. U.S. Department of Agriculture, Forest Service, Rocky Mountain Research Station, Fire Sciences Laboratory (Producer). Available: http://www.fs.fed.us/database/feis/ [2016, March 29].

Zouhar, K.L. (2002d). Cynoglossum officinale. In: Fire Effects Information System, [Online]. U.S. Department of Agriculture, Forest Service, Rocky Mountain Research Station, Fire Sciences Laboratory (Producer). Available: http://www.fs.fed.us/database/feis/ [2016, March 29].

Zouhar, K.L. (2003). Linaria spp. In: Fire Effects Information System, [Online]. U.S. Department of Agriculture, Forest Service, Rocky Mountain Research Station, Fire Sciences Laboratory (Producer). Available: http://www.fs.fed.us/database/feis/ [2016, March 29].

Zouhar, K.L. (2004). Hypericum perforatum. In: Fire Effects Information System, [Online]. U.S. Department of Agriculture, Forest Service, Rocky Mountain Research Station, Fire Sciences Laboratory (Producer). Available: http://www.fs.fed.us/database/feis/ [2016, March 29].

Zouhar, K.L. (2005a). Cytisus scoparius, C. striatus. In: Fire Effects Information System, [Online]. U.S. Department of Agriculture, Forest Service, Rocky Mountain Research Station, Fire Sciences Laboratory (Producer). Available: http://www.fs.fed.us/database/feis/ [2016, March 29].

Zouhar, K.L. (2005b). Elaeagnus angustifolia. In: Fire Effects Information System, [Online]. U.S. Department of Agriculture, Forest Service, Rocky Mountain Research Station, Fire Sciences Laboratory (Producer). Available: http://www.fs.fed.us/database/feis/ []. 
Zouhar, K.L. (2005c). Genista monspessulana. In: Fire Effects Information System, [Online]. U.S. Department of Agriculture, Forest Service, Rocky Mountain Research Station, Fire Sciences Laboratory (Producer). Available: http://www.fs.fed.us/database/feis/ [2016, March 29].

Zouhar, K.L. (2005d). Ulex europaeus. In: Fire Effects Information System, [Online]. U.S. Department of Agriculture, Forest Service, Rocky Mountain Research Station, Fire Sciences Laboratory (Producer). Available: http://www.fs.fed.us/database/feis/ [2016, March 29].

Zouhar, K.L. (2008). Berberis thunbergii. In: Fire Effects Information System, [Online]. U.S. Department of Agriculture, Forest Service, Rocky Mountain Research Station, Fire Sciences Laboratory (Producer). Available: http://www.fs.fed.us/database/feis/ [2016, March 29].

Zouhar, K.L. (2009). Isatis tinctoria. In: Fire Effects Information System, [Online]. U.S. Department of Agriculture, Forest Service, Rocky Mountain Research Station, Fire Sciences Laboratory (Producer). Available: http://www.fs.fed.us/database/feis/ [2016, March 29].

Zurell, D., Grimm, V., Rossmanith, E., Zbinden, N., Zimmermann, N.E., \& Schröder, B. (2012). Uncertainty in predictions of range dynamics: black grouse climbing the Swiss Alps. Ecography, 35(7), 590-603. 